\title{
Chemistry of Polyvalent lodine
}

\author{
Viktor V. Zhdankin and \\ Department of Chemistry and Biochemistry, University of Minnesota Duluth, Duluth, Minnesota \\ 55812 \\ Peter J. Stang \\ Department of Chemistry, 315 S 1400 E, Rm 2020, University of Utah, Salt Lake City, Utah 84112
}

\section{Introduction}

Starting from the early 1990's, the chemistry of polyvalent iodine organic compounds has experienced an explosive development. This surging interest in iodine compounds is mainly due to the very useful oxidizing properties of polyvalent organic iodine reagents, combined with their benign environmental character and commercial availability. Iodine(III) and iodine (V) derivatives are now routinely used in organic synthesis as reagents for various selective oxidative transformations of complex organic molecules. Several areas of hypervalent organoiodine chemistry have recently attracted especially active interest and research activity. These areas, in particular, include the synthetic applications of 2-iodoxybenzoic acid (IBX) and similar oxidizing reagents based on the iodine $(\mathrm{V})$ derivatives, the development and synthetic use of polymer-supported and recyclable polyvalent iodine reagents, the catalytic applications of organoiodine compounds, and structural studies of complexes and supramolecular assemblies of polyvalent iodine compounds.

The chemistry of polyvalent iodine has previously been covered in four books ${ }^{1-4}$ and several comprehensive review papers. ${ }^{5-17}$ Numerous reviews on specific classes of polyvalent iodine compounds and their synthetic applications have recently been published. ${ }^{18-61}$ Most notable are the specialized reviews on [hydroxy(tosyloxy)iodo]benzene, ${ }^{41}$ the chemistry and synthetic applications of iodonium salts, ${ }^{29,36,38,42,43,46,47,54,55}$ the chemistry of iodonium ylides, ${ }^{56-}$ 58 the chemistry of iminoiodanes, ${ }^{28}$ hypervalent iodine fluorides,,${ }^{27}$ electrophilic perfluoroalkylations, ${ }^{44}$ perfluoroorgano hypervalent iodine compounds,${ }^{61}$ the chemistry of benziodoxoles ${ }^{24,45}$ polymer-supported hypervalent iodine reagents, ${ }^{30}$ hypervalent iodinemediated ring contraction reactions, ${ }^{21}$ application of hypervalent iodine in the synthesis of heterocycles ${ }^{25,40}$ application of hypervalent iodine in the oxidation of phenolic compounds, $32,34,50-53,60$ oxidation of carbonyl compounds with organohypervalent iodine reagents, ${ }^{37}$ application of hypervalent iodine in (hetero)biaryl coupling reactions, ${ }^{31}$ phosphorolytic reactivity of $o$-iodosylcarboxylates, ${ }^{33}$ coordination of hypervalent iodine, ${ }^{19}$ transition metal catalyzed reactions of hypervalent iodine compounds, ${ }^{18}$ radical reactions of hypervalent iodine, ${ }^{35,39}$ stereoselective reactions of hypervalent iodine electrophiles, ${ }^{48}$ catalytic applications of organoiodine compounds, ${ }^{20,49}$ and synthetic applications of pentavalent iodine reagents. ${ }^{22,23,26,59}$

The main purpose of the present review is to summarize the data that appeared in the literature following publication of our previous reviews in 1996 and 2002. In addition, a brief introductory discussion of the most important earlier works is provided in each section. The 
review is organized according to the classes of organic polyvalent iodine compounds with emphasis on their synthetic application. Literature coverage is through July 2008.

\section{Structure and Bonding}

\subsection{General Features}

Structural aspects of polyvalent iodine compounds have previously been discussed in our original 1996 review $^{5}$ and in the 1992 monograph by Varvoglis. ${ }^{2}$ More recently, general aspects of structure and bonding in hypervalent organic compounds have been summarized by Akiba in the book on Chemistry of Hypervalent Compounds ${ }^{62}$ and by Ochiai in a chapter in the volume on Hypervalent Iodine Chemistry in Topics in Current Chemistry. ${ }^{1}$ A brief summary of the key structural features of iodine(III) and iodine(V) compounds is provided below.

All known organic polyvalent iodine derivatives belong to two general structural types: (1) iodine(III) compounds $\mathbf{1}$ and $\mathbf{2}$, also named $\lambda^{3}$-iodanes according to IUPAC recommendations, and (2) iodine(V) compounds $\mathbf{3}$, or $\lambda^{5}$-iodanes. The iodine atom in $\lambda^{3}$-iodanes $\mathbf{1}$ has a total 10 electrons and the overall geometry of a distorted trigonal bipyramid with two heteroatom ligands $\mathrm{X}$ occupying the apical positions, and the least electronegative carbon ligand $\mathrm{R}$ and both electron pairs residing in equatorial positions. Iodonium salts $\mathbf{2}$, which have two carbon ligands and a closely associated anionic part of the molecule, have a similar pseudo trigonal bipyramidal geometry and also belong to $\lambda^{3}$-iodanes. In agreement with this model, the experimentally determined bond angle R-I-R in iodonium salts and ylides is close to $90^{\circ}$. In the hypervalent model, bonding in $\mathrm{RIX}_{2}$ uses the non-hybridized $5 \mathrm{p}$ orbital of iodine in the linear X-I-X bond. Such a linear three-center, four-electron ( $3 \mathrm{c}-4 \mathrm{e})$ bond is highly polarized and is longer and weaker compared to a regular covalent bond. This bond is termed "hypervalent" and the presence of this bond in $\lambda^{3}$-iodanes is responsible for their high electrophilic reactivity.

Organic $\lambda^{5}$-iodanes 3 have a distorted octahedral structure with the organic group $\mathrm{R}$ and the electron pair in the apical positions and four heteroatom ligands $\mathrm{X}$ in basal positions. Two orthogonal hypervalent $3 \mathrm{c}-4 \mathrm{e}$ bonds accommodate all ligands $\mathrm{X}$, while the apical group $\mathrm{R}$ is connected to iodine by a normal covalent bond using 5 sp-hybridized orbital. ${ }^{2}$ In general, only $\lambda^{3}$ - and $\lambda^{5}$-iodanes with an aromatic group $\mathrm{R}(\mathrm{R}=$ aryl or hetaryl $)$ have sufficient stability and can be isolated. A few examples of alkyl substituted $\lambda^{3}$-iodanes stabilized by strong electronwithdrawing groups (perfluoroalkyl or arylsulfonylmethyl $\lambda^{3}$-iodanes) have also been isolated. The stable aryl substituted $\lambda^{3}$ - and $\lambda^{5}$-iodanes possess high chemical reactivity and are widely used in organic synthesis as oxidants and electrophilic agents, which are commonly referred to as "hypervalent iodine reagents".
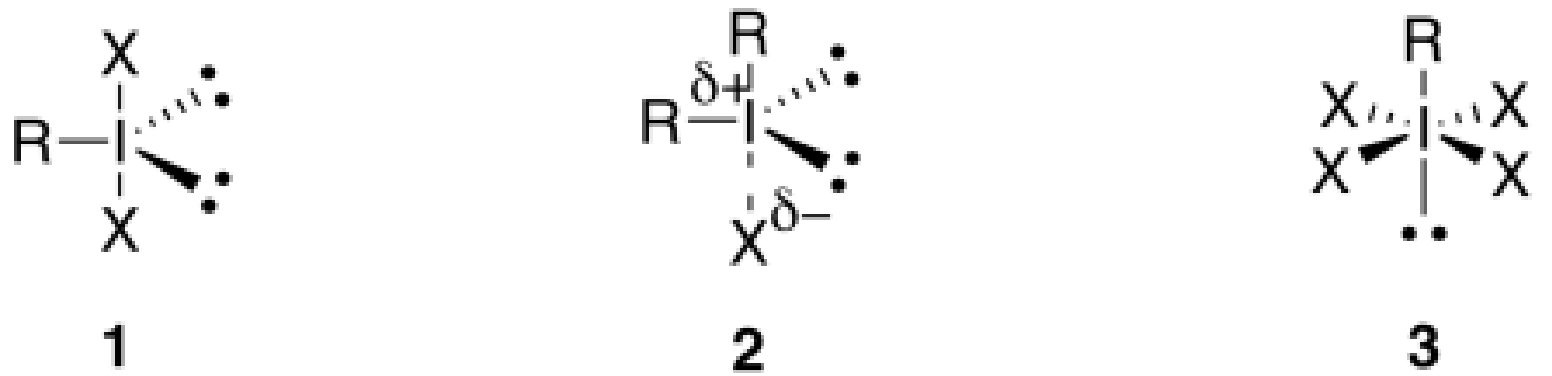

\section{$\mathrm{R}=$ carbon ligand; $\mathrm{X}=$ halogen, $\mathrm{O}-$, or $\mathrm{N}$-ligand}




\subsection{Computational Studies}

A relatively small number of theoretical computational studies concerning the structure and reactivity of hypervalent iodine compounds have appeared in the last 10 years. ${ }^{63-76}$ Hoffmann and co-workers analyzed the nature of hypervalent bonding in trihalide anions by applying ideas from qualitative MO theory to computational results from density-functional calculations. ${ }^{63}$ This systematic, unified investigation showed that the bonding in all of these systems can be explained in terms of the Rundle-Pimentel scheme for electron-rich three-center bonding. The same authors reported an analysis of intermolecular interaction between hypervalent molecules, including diaryliodonium halides $\mathrm{Ar}_{2} \mathrm{IX}$, using a combination of density functional calculations and qualitative arguments. ${ }^{64}$ Based on fragment molecular orbital interaction diagrams, the authors concluded that the secondary bonding in these species can be understood using the language of donor-acceptor interactions: mixing between occupied states on one fragment and unoccupied states on the other. There is also a strong electrostatic contribution to the secondary bonding. The calculated strengths of these halogen-halogen secondary interactions are all less than $10 \mathrm{kcal} \mathrm{mol}^{-164}$

The self-assembly of hypervalent iodine compounds to macrocyclic trimers was studied using MO calculations. The principal driving force for the self-assembly of iodonium units is the formation of secondary bonding interactions between iodonium units as well as a rearrangement of primary and secondary bonding around iodine to place the least electronegative substituent in the equatorial position for every iodine in the trimer. ${ }^{65}$

Kiprof has analyzed the iodine oxygen bonds of hypervalent 10-I-3 iodine(III) compounds with $\mathrm{T}$-shaped geometry using the Cambridge Crystallographic Database and ab initio MO calculations. The statistical analysis of the $\mathrm{I}-\mathrm{O}$ bond lengths in $\mathrm{PhI}(\mathrm{OR})_{2}$ revealed an average of $2.14 \AA$ and a strong correlation between the two bond lengths. ${ }^{66}$ Further theoretical investigation of the mutual ligand interaction in the hypervalent $\mathrm{L}-\mathrm{I}-\mathrm{L}^{\prime}$ system has demonstrated that ligands' trans influences play an important role in the stability of hypervalent molecules. ${ }^{67}$ In particular, combinations of ligands with large and small trans influences, as in $\mathrm{PhI}(\mathrm{OH}) \mathrm{OTs}$, or of two moderately trans influencing ligands, as in $\mathrm{PhI}(\mathrm{OAc})_{2}$, are favored and lead to higher stability of the molecule. trans Influences also seem to explain why iodosylbenzene, $(\mathrm{PhIO})_{\mathrm{n}}$, adopts an oxo-bridged zigzag polymer structure in contrast to $\mathrm{PhI}$ $(\mathrm{OH})_{2}$, which is monomeric. ${ }^{67}$

The structure and reactivity of several specific classes of hypervalent iodine compounds were theoretically investigated. In particular, Okuyama and Yamataka investigated the reactivity of vinyliodonium ions with nucleophiles by ab initio MO (MP2) calculations at the double-zeta $(\mathrm{DZ})+\mathrm{d}$ level. ${ }^{68}$ It was proposed that interaction of methyl(vinyl)iodonium ion with chlorine anion leads to chloro- $\lambda^{3}$-iodane $\mathrm{CH}_{2}=\mathrm{CHI}(\mathrm{Me}) \mathrm{Cl}$. Transition states for the $\mathrm{S}_{\mathrm{N}} 2$, ligandcoupling substitution, and $\beta$-elimination were found for reactions at the vinyl group. The barrier to ligand-coupling substitution is usually the lowest in the gas phase, but relative barriers to $\mathrm{S}_{\mathrm{N}} 2$ and to $\beta$-elimination change with the substituents. Effects of solvent on this reaction were evaluated by a dielectric continuum model and found to be large on $\mathrm{S}_{\mathrm{N}} 2$ but small on ligandcoupling. ${ }^{68}$

Widdowson, Rzepa and co-workers reported $a b$ initio and MNDO-d SCF-MO computational studies of the extrusion reactions of diaryliodonium fluorides. ${ }^{69,71}$ The results of these studies, in particular, predicted that the intermediates and transition states in these reactions might involve dimeric, trimeric, and tetrameric structures. The regioselectivity of nucleophilic substitution in these reactions was investigated theoretically and supported by some experimental observations. ${ }^{69-71}$ 
Goddard and Su have theoretically investigated the mechanism of alcohol oxidation with 2iodoxybenzoic acid (IBX) on the basis of density functional quantum mechanics calculations. ${ }^{72}$ It has been found that the rearrangement of hypervalent bonds, so called hypervalent twisting, is the rate-determining step in this reaction. Based on this mechanism, the authors explain why IBX oxidizes large alcohols faster than small ones and propose a modification to the reagent predicted to make it more active. ${ }^{72}$

Bakalbassis, Spyroudis, and Tsiotra reported a DFT study on the intramolecular thermal phenyl migration in iodonium ylides. The results of this study support a single-step mechanism involving a five-membered ring transition-state. The frontier-orbital-controlled migration also confirms the different thermal behavior experimentally observed for two different ylides. ${ }^{77}$

Molecular orbital computational studies of (arylsulfonylimino)iodoarenes $\left(\mathrm{ArINSO}_{2} \mathrm{Ar}^{\prime}\right){ }^{73}$ benziodazol-3-ones, ${ }^{74}$ and a series of ortho-substituted chiral organoiodine(III) compounds ${ }^{75}$ have been reported in the literature. Results of these calculations were found to be in good agreement with X-ray structural data for these compounds.

In a very recent communication, Quideau and co-workers presented DFT calculations of spiroheterocylic iodine(III) intermediates to validate their participation in the $\mathrm{PhI}(\mathrm{OAc})_{2}$ mediated spiroketalization of phenolic alcohols. ${ }^{76}$

\subsection{Experimental Structural Studies}

Numerous X-ray crystal structures have been reported for all main classes of organic polyvalent iodine compounds, and the results of these studies will be briefly discussed in the appropriate sections of this review. Several general areas of structural research on hypervalent organoiodine compounds have recently attracted especially active interest. These areas, in particular, include the preparation and structural study of complexes of hypervalent iodine compounds with crown ethers ${ }^{78-82}$ or nitrogen ligands, ${ }^{83-85}$ self-assembly of hypervalent iodine compounds into various supramolecular structures, ${ }^{86-88}$ and the intramolecular secondary bonding in ortho-substituted aryliodine(V) and iodine(III) derivatives. ${ }^{73,89-99}$

Typical coordination patterns in various organic derivatives of iodine(III) in the solid state with consideration of primary and secondary bonding have been summarized by Sawyer and coworkers ${ }^{100}$ in 1986 and updated in recent publications. ${ }^{101-104}$ Structural features of organic iodine(V) compounds have been discussed in older papers of Martin and co-authors, ${ }^{105,106}$ and in numerous more recent publications on IBX and related $\lambda^{5}$-iodanes. ${ }^{89,93-98,107}$

Several important spectroscopic structural studies of polyvalent iodine compounds in the solution have been published. ${ }^{108-112}$ Hiller and co-workers reported NMR and LC-MS study on the structure and stability of 1-iodosyl-4-methoxybenzene and 1-iodosyl-4-nitrobenzene in methanol solution. ${ }^{108}$ Interestingly, LC-MS analyses provided evidence that unlike the parent iodosylbenzene, which has a polymeric structure, the 4-substituted iodosylarenes exist in the monomeric form. Both iodosylarenes are soluble in methanol and provide acceptable ${ }^{1} \mathrm{H}$ and ${ }^{13} \mathrm{C}$ NMR spectra; however, gradual oxidation of the solvent was observed after several hours. Unlike iodosylbenzene, the two compounds did not react with methanol to give the dimethoxy derivative $\operatorname{ArI}(\mathrm{OMe})_{2} .{ }^{108}$

Cerioni, Mocci and co-workers investigated the structure of bis(acyloxy)iodoarenes and benzoiodoxolones in chloroform solution by ${ }^{17} \mathrm{O}$ NMR spectroscopy and also by DFT calculations. ${ }^{109,110}$ This investigation provided substantial evidence that the T-shaped structure of iodine(III) compounds observed in the solid state is also adopted in solution. Furthermore, the "free" carboxylic groups of bis(acyloxy)iodoarenes show a dynamic behavior, observable only in the ${ }^{17} \mathrm{O}$ NMR. This behavior is ascribed to a $\left[{ }^{1,3}\right]$ sigmatropic 
shift of the iodine atom between the two oxygen atoms of the carboxylic groups, and the energy involved in this process varies significantly between bis(acyloxy)iodoarenes and benzoiodoxolones. ${ }^{110}$

Richter, Koser and co-workers investigated the nature of species present in aqueous solutions of phenyliodine(III) organosulfonates. ${ }^{111}$ It was shown by spectroscopic measurements and potentiometric titrations that $\mathrm{PhI}(\mathrm{OH}) \mathrm{OTs}$ and $\mathrm{PhI}(\mathrm{OH}) \mathrm{OMs}$ upon solution in water undergo complete ionization to give the hydroxy(phenyl)iodonium ion $\left(\mathrm{PhI}{ }^{+} \mathrm{OH}\right.$ in hydrated form) and the corresponding sulfonate ions. The hydroxy(phenyl)iodonium ion can combine with [oxo (aquo)iodo]benzene $\mathrm{PhI}^{+}\left(\mathrm{OH}_{2}\right) \mathrm{O}^{-}$, a hydrated form of iodosylbenzene that is also observed in the solution, producing the dimeric $\mu$-oxodiiodine cation $\mathrm{Ph}(\mathrm{HO}) \mathrm{I}-\mathrm{O}-\mathrm{I}^{+}\left(\mathrm{OH}_{2}\right) \mathrm{Ph}$ and dication $\mathrm{Ph}\left(\mathrm{H}_{2} \mathrm{O}\right) \mathrm{I}^{+}-\mathrm{O}-\mathrm{I}^{+}\left(\mathrm{OH}_{2}\right) \mathrm{Ph} .{ }^{111}$

Silva and Lopes analyzed solutions of iodobenzene dicarboxylates in acetonitrile, acetic acid, aqueous methanol and anhydrous methanol by electrospray ionization mass spectrometry (ESIMS) and tandem mass spectrometry (ESI-MS/MS). ${ }^{112}$ The major species found in the solutions of $\mathrm{PhI}(\mathrm{OAc})_{2}$ in acetonitrile, acetic acid, and aqueous methanol are $\left[\mathrm{PhI}(\mathrm{OAc})_{2} \mathrm{Na}\right]^{+},[\mathrm{PhI}$ $\left.(\mathrm{OAc})_{2} \mathrm{~K}\right]^{+},[\mathrm{PhI}]^{+},[\mathrm{PhIOAc}]^{+},[\mathrm{PhIOH}]^{+},\left[\mathrm{PhIO}_{2} \mathrm{Ac}\right]^{+},\left[\mathrm{PhIO}_{2} \mathrm{H}\right]^{+}$and the dimer $\left[\mathrm{Ph}_{2} \mathrm{I}_{2} \mathrm{O}_{2} \mathrm{Ac}\right]^{+}$. On the other hand, the anhydrous methanol solutions showed [PhIOMe $]^{+}$as the most abundant species. In contrast to the data obtained for $\mathrm{PhI}(\mathrm{OAc})_{2}$, the ESI-MS spectral data of $\mathrm{PhI}\left(\mathrm{O}_{2} \mathrm{CCF}_{3}\right)_{2}$ in acetonitrile suggests that the main species in solutions is iodosylbenzene. ${ }^{112}$

\section{3. lodine(III) Compounds}

Iodine(III) compounds (structures $\mathbf{1}$ and $\mathbf{2}$ ), or $\lambda^{3}$-iodanes according to the IUPAC nomenclature, are commonly classified by the type of ligands attached to the iodine atom. ${ }^{2,3}$, 5,6 This section of the review is organized according to the traditional classification and will cover the preparation, structure, and reactivity of iodosylarenes, aryliodine(III) halides, carboxylates, sulfonates, cyclic $\lambda^{3}$-iodanes, iodonium salts, ylides, and imides with emphasis on their synthetic application.

\section{1. lodosylarenes}

3.1.1. Preparation-The most important representative of iodosylarenes, iodosylbenzene, is best prepared by alkaline hydrolysis of (diacetoxy)iodobenzene. ${ }^{113}$ The same procedure can be used for the preparation of a variety of ortho-, meta-, and para-substituted iodosylbenzenes from the respective (diacetoxy)iodoarenes (Scheme 1). ${ }^{90-92,108,114}$ This procedure, for example, was recently used for the preparation of 4-methoxyiodosylbenzene, ${ }^{108} 4$ nitroiodosylbenzene ${ }^{108}$ and pseudocyclic iodosylarenes bearing tert-butylsulfonyl ${ }^{91}$ or diphenylphosphoryl ${ }^{92}$ groups in the ortho-position.

An alternative general procedure for the preparation of iodosylarenes $\mathbf{7}$ employs the alkaline hydrolysis of (dichloroiodo)arenes under conditions similar to the hydrolysis of (diacetoxyiodo)arenes. ${ }^{115}$ A modified procedure employs aqueous tetrahydrofuran as the solvent for the hydrolysis of (dichloroiodo)arenes 6 (Scheme 2). ${ }^{116}$

Iodosylbenzene is a yellowish amorphous powder, which cannot be recrystallized due to its polymeric nature; it dissolves in methanol with depolymerization affording $\mathrm{PhI}(\mathrm{OMe})_{2} .{ }^{117}$ Heating or extended storage at room temperature results in disproportionation of iodosylbenzene to $\mathrm{PhI}$ and a colorless, explosive iodylbenzene, $\mathrm{PhIO}_{2}$. Drying iodosylbenzene at elevated temperatures should be avoided; a violent explosion of $3.0 \mathrm{~g}$ PhIO upon drying at $110{ }^{\circ} \mathrm{C}$ in vacuum has recently been reported. ${ }^{118}$ 
3.1.2. Structural Studies-Based on spectroscopic studies, it was suggested that in the solid state iodosylbenzene exists as a zigzag polymeric, asymmetrically bridged structure, in which monomeric units of $\mathrm{PhIO}$ are linked by intermolecular $\mathrm{I} \cdots 0 \mathrm{O}$ secondary bonds. ${ }^{6}$ The IO bond distances of 2.04 and $2.37 \AA$ and the $\mathrm{C}-\mathrm{I}-\mathrm{O}$ bond angle near $90^{\circ}$ have been deduced from EXAFS analysis of polymeric iodosylbenzene. ${ }^{119}$ The polymeric structure of iodosylbenzene was also theoretically analyzed by density functional theory computations at the B3LYP level and, in particular, the importance of the presence of a terminal hydration water in its zigzag polymeric structure $\mathrm{HO}-(\mathrm{PhIO})_{\mathrm{n}}-\mathrm{H}$ was established. ${ }^{120}$ The zigzag asymmetrically bridged structure of $(\mathrm{PhIO})_{\mathrm{n}}$ has recently been confirmed by single crystal Xray diffraction studies of the oligomeric sulfate $\mathbf{8}$ and perchlorate $\mathbf{9}$ derivatives. ${ }^{87,121}$ In particular, iodine atoms in the $(\mathrm{PhIO})_{3}$ fragment of the oligomeric sulfate 8 exhibit a typical of trivalent iodine T-shaped intramolecular geometry with O-I-O and O-I-C bond angles close to $180^{\circ}(166.54-177.99)$ and $90^{\circ}$ (79.18-92.43), respectively. The I-O bond distances in the $(\mathrm{PhIO})_{3}$ fragment of sulfate 8 vary in a broad range of 1.95 to $2.42 \AA .{ }^{121}$ The single crystal Xray crystal study of the oligomeric perchlorate 9 revealed a complex structure consisting of pentaiodanyl dicationic units joined by secondary $\mathrm{I} \bullet \bullet \mathrm{O}$ bonds into an infinite linear structure of 12 -atom hexagonal rings. ${ }^{87}$ The oligomer 8 was prepared by the treatment of $\mathrm{PhI}(\mathrm{OAc})_{2}$ with aqueous $\mathrm{NaHSO}_{4}$, while product 9 precipitated from dilute aqueous solutions of $\mathrm{PhI}(\mathrm{OH})$ OTs and $\mathrm{Mg}\left(\mathrm{ClO}_{4}\right)_{2}$. The formation of both products can be explained by self-assembly of the hydroxy(phenyl)iodonium ions $\left(\mathrm{PhI}^{+} \mathrm{OH}\right.$ in hydrated form) and [oxo(aquo)iodo]benzene $\mathrm{PhI}^{+}\left(\mathrm{OH}_{2}\right) \mathrm{O}^{-}$in aqueous solution under reaction conditions.

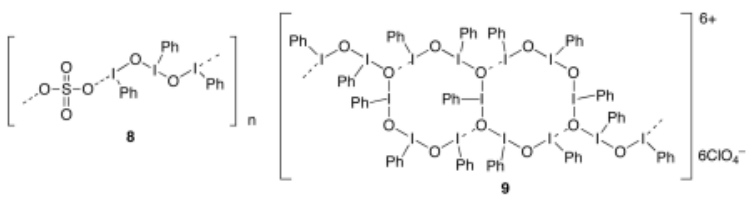

Ochiai and co-workers have reported the preparation, X-ray crystal structures, and useful oxidizing reactions of activated iodosylbenzene monomer complexes with $18 \mathrm{C} 6$ crown ether. 19,78 Reaction of iodosylbenzene with $\mathrm{HBF}_{4}-\mathrm{Me}_{2} \mathrm{O}$ in the presence of equimolar $18 \mathrm{C} 6$ in dichloromethane afforded quantitatively the stable, crystalline crown ether complex 10, which is soluble in $\mathrm{MeCN}, \mathrm{MeOH}$, water, and dichloromethane. X-ray analysis revealed a protonated iodosylbenzene monomer structure $\mathbf{1 0}$ stabilized by intramolecular coordination with the crown ether oxygen atoms. ${ }^{78}$ The aqua complexes of iodosylarenes $\mathbf{1 1}$ and $\mathbf{1 2}$ with a water molecule coordinated to iodine(III) were prepared by the reaction of (diacetoxyiodo)benzene with trimethylsilyl triflate in the presence of $18 \mathrm{C} 6$ crown ether in dichloromethane. X-ray analysis of complex $\mathbf{1 1}$ revealed a T-shaped structure, ligated with one water molecule at the apical site of the iodine(III) atom of hydroxy(phenyl)iodonium ion, with a near-linear O-I-O triad (173.96). Including a close contact with one of the crown ether oxygens, the complex adopts a distorted square planar geometry around the iodine. ${ }^{122}$

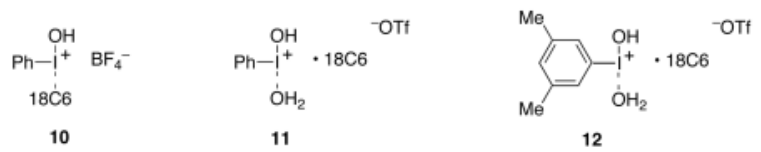

The ortho-substituted iodosylarenes 13-16 bearing tert-butylsulfonyl, ${ }^{91}$ diphenylphosphoryl, ${ }^{92}$ or nitro ${ }^{99}$ groups have a monomeric, pseudocyclic structure due to the replacement of intermolecular $\mathrm{I} \cdots \mathrm{O}$ interactions with intramolecular secondary bonding. The structure of product $\mathbf{1 3}$ was established by single crystal X-ray analysis. ${ }^{89}$ 


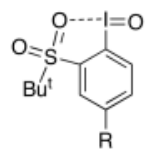

$13(\mathrm{R}=\mathrm{H})$

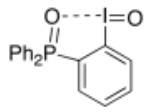

15

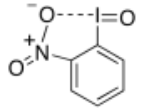

16

3.1.3. Oxidations with lodosylarenes-Iodosylbenzene is an effective oxidizing reagent but its insolubility, due to the polymeric structure, significantly restricts its practical usefulness. The overwhelming majority of the known reactions of iodosylbenzene require the presence of a hydroxylic solvent (water or alcohols) or a catalyst (Lewis acid, bromide or iodide anions, transition metal complex, etc.) that can effectively depolymerize $(\mathrm{PhIO})_{\mathrm{n}}$ generating the reactive monomeric species. Numerous examples of such oxidations have been reported in our previous reviews ${ }^{5,6}$ and include, for example, selective oxidation of alcohols ${ }^{123,124}$ or sulfides ${ }^{125}$ with $(\mathrm{PhIO})_{\mathrm{n}} / \mathrm{KBr} / \mathrm{H}_{2} \mathrm{O}$, the oxidation of silyl enol ethers to $\alpha$-hydroxy- and $\alpha$ alkoxy substituted of carbonyl compounds using $(\mathrm{PhIO})_{\mathrm{n}} / \mathrm{BF}_{3} \cdot \mathrm{Et}_{2} \mathrm{O}$ in water or an alcohol, 126,127 the generation and sequential fragmentation of radicals from alcohols or amides (e.g., 17 and 18) with the PhIO- $\mathrm{I}_{2}$ system (Scheme 3), ${ }^{128-130}$ and the oxidation of tetrahydroisoquinolines 19 by $(\mathrm{PhIO})_{n} / \mathrm{Bu}_{4} \mathrm{NI} / \mathrm{H}_{2} \mathrm{O}$ to the respective lactams 20 (Scheme 4). 131

Several new oxidations with $(\mathrm{PhIO})_{\mathrm{n}}$ have been recently reported. The oxidation of 3hydroxypiperidine $\mathbf{2 1}$ with iodosylbenzene in water affords 2-pyrrolidinone $\mathbf{2 2}$ directly in good yield (Scheme 5). ${ }^{132}$ The mechanism of this reaction probably involves oxidative Grob fragmentation yielding imino aldehyde, which upon hydrolysis affords 2-pyrrolidinone by a cyclization-oxidation sequence.

Togo and co-workers have reported the preparation of $\alpha$-tosyloxy ketones and aldehydes 24 in good yields from alcohols $\mathbf{2 3}$ by treatment with iodosylbenzene and $p$-toluenesulfonic acid monohydrate. This method can also be used for the direct preparation of thiazoles $(\mathbf{2 5}, X=S)$, imidazoles $(\mathbf{2 5}, \mathrm{X}=\mathrm{NH})$, and imidazo[1,2-a]pyridines $\mathbf{2 6}$ from alcohols in good to moderate yields by the successive treatment with iodosylbenzene and $p$-toluenesulfonic acid monohydrate, followed by thioamides, benzamidine, and 2-aminopyridine, respectively (Scheme 6). ${ }^{133}$

The reactions of 4-acyloxybut-1-enylsilanes 27 with iodosylbenzene in the presence of $\mathrm{BF}_{3} \cdot \mathrm{OEt}_{2}$ afford 4-acyloxy-2-oxobutylsilanes $\mathbf{2 8 ,} \mathbf{3 1}$ and 3-acyloxytetrahydrofuran-2ylsilanes 29, 32 via a 1,3-dioxan-2-yl cation intermediate, which is generated by participation of the acyloxy group during the electrophilic addition of iodine(III) species to the substrate (Scheme 7). ${ }^{134}$

Ochiai and co-workers have reported several useful oxidations employing the activated iodosylbenzene species. ${ }^{19,78,122,135,136}$ The monomeric iodosylbenzene complex $\mathbf{1 0}$ in the presence of water can cleave the carbon-carbon double bond of indene $\mathbf{3 3}$ with the formation of dialdehyde 34 (Scheme 8). ${ }^{135}$ Similar oxidative cleavage of various alkenes can be performed by using iodosylbenzene in water in the presence of $\mathrm{HBF}_{4}$. This convenient procedure provides a safe alternative to the ozonolysis of alkenes. ${ }^{135}$

Reaction of 3-phenylpropanol $\mathbf{3 5}$ with activated iodosylbenzene complex $\mathbf{1 0}$ in dichloromethane in the presence of $\mathrm{BF}_{3} \cdot \mathrm{OEt}_{2}$ afforded directly the 6-chromanyl(phenyl) iodonium salt 36 (isolated as a complex with $18 \mathrm{C} 6$ crown ether) through tandem oxidative intramolecular cyclization yielding chroman and its subsequent regioselective reaction with complex 10 leading to the final product 36 (Scheme 9). ${ }^{136}$ 
The oligomeric iodosylbenzene sulfate $(\mathrm{PhIO})_{3} \bullet \mathrm{SO}_{3}$ (structure $\mathbf{8}$ ) is a readily available, stable, and water-soluble reagent with reactivity pattern similar to activated iodosylbenzene. It reacts with alkenes, alcohols, and aryl alkyl sulfides in aqueous acetonitrile at room temperature to afford the respective products of oxidation $\mathbf{3 7 - 4 0}$ in good yields (Scheme 10). ${ }^{88}$

Iodosylbenzene is a useful reagent for nucleophilic epoxidation of electron-deficient alkenes, such as tetrasubstituted perfluoroalkenes ${ }^{137}$ and $\alpha, \beta$-unsaturated carbonyl compounds. ${ }^{118,138}$ In a specific example, iodosylbenzene reacts with enones $\mathbf{4 1}$ to furnish the corresponding epoxides $\mathbf{4 2}$ in generally high yields (Scheme 11). ${ }^{118}$

Only very few ArIO other than iodosylbenzene have been used as reagents. The only exception is represented by ortho- and meta-iodosylbenzoic acids. The $o$-iodosylbenzoic acid (IBA) has a cyclic structure of benziodoxolone and is discussed in Section 3.7 of this review. The $m$ iodosylbenzoic acid has recently found some synthetic application as an efficient, safe, and recyclable oxidant. ${ }^{103,139,140}$ In particular, $m$-iodosylbenzoic acid in the presence of iodine is a convenient reagent for oxidative iodination of arenes at room temperature in acetonitrile solution. Separation of pure products is conveniently achieved by scavenging any aryl iodide by ion exchange with ion exchange resin IRA-900 (hydroxide form). The reduced form of the reagent, $m$-iodobenzoic acid, can be easily recovered from the ion exchange resin or from the basic aqueous solution by simple acidification with $\mathrm{HCl} .{ }^{140}$

3.1.3. Transition Metal Catalyzed Oxidations-The oxidation reactions of iodosylarenes can be effectively catalyzed by metal salts and complexes. ${ }^{6}$ Iodosylbenzene is widely used as the most efficient terminal oxidant - source of oxygen in biomimetic oxidations catalyzed by metalloporphyrins and other transition metal derivatives. ${ }^{141-145}$ Recent examples of transition metal catalyzed oxidations employing iodosylbenzene include the hydroxylation of hydrocarbons, ${ }^{146-151}$ the transition metal-mediated epoxidation of alkenes, ${ }^{138,152-169}$ oxidation of alcohols ${ }^{170,171}$ or silyl ethers ${ }^{172}$ to carbonyl compounds, $\delta$-sultone formation through Rh-catalyzed C-H insertion, ${ }^{173}$ and oxidation of organic sulfides ${ }^{163,174,175}$ to sulfoxides.

Iodosylarenes other than iodosylbenzene have also been used in the transition metal catalyzed oxidation reactions. The soluble, monomeric ortho-substituted iodosylarene $\mathbf{1 3}$ (see Section 3.1.2) can serve as an alternative to iodosylbenzene in the (porphyrin)manganese(III)-catalyzed alkene epoxidation reactions. ${ }^{157} \mathrm{~A}$ convenient recyclable reagent, $m$-iodosylbenzoic acid, selectively oxidizes primary and secondary alcohols to the respective carbonyl compounds in the presence of $\mathrm{RuCl}_{3}(0.5 \mathrm{~mol} \%)$ at room temperature in aqueous acetonitrile. ${ }^{139}$ Separation of pure products in this case is achieved by simple extraction of the basic aqueous solution, and the reduced form of the reagent, $m$-iodobenzoic acid, can be easily recovered from the aqueous solution by simple acidification.

\subsection{Fluorides}

3.2.1. Preparation-A clean and selective, although relatively expensive procedure for the preparation of (difluoroiodo)arenes $\mathbf{4 3}$ consists of the treatment of iodoarenes with xenon difluoride in dichloromethane (Scheme 12) in the presence of anhydrous hydrogen fluoride. 176,177 This method works well for the fluorination of iodoarenes with electron-donating or electron-withdrawing substituents; the latter, however, require longer reaction times. (Difluoroiodo)arenes $\mathbf{4 3}$ are hygroscopic and highly hydrolizable compounds, which make their separation and crystallization extremely difficult. Since xenon is the only byproduct in this reaction (Scheme 12), the resulting dichloromethane solutions contain essentially pure fluorides $\mathbf{4 3}$ which can be used in the subsequent reactions without additional purification. A similar procedure, but in the absence of anhydrous hydrogen fluoride, has been employed in the synthesis of some heteroaromatic iododifluorides. 2,3,5,6-Tetrafluoropyridin-4-yliodine 
difluoride, 4- $\left(\mathrm{C}_{5} \mathrm{~F}_{4} \mathrm{~N}\right) \mathrm{IF}_{2}$ was prepared in $84 \%$ yield from by the reaction of $4-\left(\mathrm{C}_{5} \mathrm{~F}_{4} \mathrm{~N}\right) \mathrm{I}$ with $\mathrm{XeF}_{2}$ in dichloromethane at room temperature. ${ }^{178}$ Likewise, the fluorination of 3-iodo-4methylfurazan with xenon difluoride in acetonitrile at room temperature was recently used for the preparation 3-(difluoroiodo)-4-methylfurazan. ${ }^{179}$

A variety of other powerful fluorinating reagents, such as $\mathrm{F}_{2}, \mathrm{ClF}, \mathrm{CF}_{3} \mathrm{OCl}, \mathrm{BrF}_{5}, \mathrm{C}_{6} \mathrm{~F}_{5} \mathrm{BrF}_{2}$, $\mathrm{C}_{6} \mathrm{~F}_{5} \mathrm{BrF}_{4}, \mathrm{XeF}_{2} / \mathrm{BF}_{3}$, can be used for the preparation of (difluoroiodo)arenes derived from polyfluorosubstituted iodoarenes. ${ }^{180-182}$ A convenient procedure for the preparation of (difluoroiodo)benzene and 4-(difluoroiodo)toluene consists of direct fluorination of the respective iodoarenes with the commercially available fluorinating reagent Selectfluor in acetonitrile solution. ${ }^{183}$ Various mixed (fluoroiodo)arene triflates, ArIF(OTf), can be generated in situ by fluorination of the respective iodoarenes with xenon fluorotriflate, FXeOTf. 184,185

The para-substituted (difluoroiodo)arenes can be effectively prepared by the electrochemical fluorination of the respective iodoarenes. ${ }^{186,187}$ In this procedure, the electrosynthesis of $\mathrm{ArIF}_{2}$ is accomplished by the anodic oxidation of iodoarenes with $\mathrm{Et}_{3} \mathrm{~N} \cdot 3 \mathrm{HF}$ or $\mathrm{Et}{ }_{3} \mathrm{~N} \cdot 5 \mathrm{HF}$ in anhydrous acetonitrile using a divided cell. This procedure works especially well for the preparation of 4- $\mathrm{NO}_{2} \mathrm{C}_{6} \mathrm{H}_{4} \mathrm{IF}_{2}$, which precipitates from the electrolytic solution in pure form during the electrolysis. The other para-substituted (difluoroiodo)arenes, such as TolIF 2 and 4$\mathrm{MeOC}_{6} \mathrm{H}_{4} \mathrm{IF}_{2}$, can be generated similarly and used without isolation as in-cell mediators for the following reactions. ${ }^{186,187}$

An older common procedure for the preparation of (difluoroiodo)arenes involves a one-step reaction of mercuric oxide and aqueous hydrofluoric acid with the (dichloroiodo)arenes in dichloromethane. ${ }^{188}$ The resulting solution of (difluoroiodo)arenes in dichloromethane can be used in the subsequent reactions without additional purification. A drawback of this method is the use of a large quantity of harmful $\mathrm{HgO}$ in order to remove the chloride ion from the reaction mixture. A convenient modified procedure without the use of $\mathrm{HgO}$ consists of the treatment of iodosylarenes 44 with 40-46\% aqueous hydrofluoric acid (Scheme 13) followed by crystallization of products $\mathbf{4 5}$ from hexane. ${ }^{116,189}$ It is important that the freshly prepared iodosylarenes $\mathbf{4 4}$ are used in this procedure.

3.2.2. Structural Studies-Only a few examples of structural studies of organoiododifluorides, $\mathrm{RIF}_{2}$, have been reported in the literature. Single crystal X-ray diffraction studies of trifluoromethyliododifluoride, $\mathrm{CF}_{3} \mathrm{IF}_{2}$, revealed a distorted $\mathrm{T}$-shaped structure with the I-F bond lengths 1.982(2) $\AA$, and the F-I-F angle $165.4(2)^{\circ}{ }^{190}$ Theoretical studies of $\mathrm{CF}_{3} \mathrm{IF}_{2}$ by ab initio and DFT calculations have also been reported. ${ }^{191}$ The structure of pentafluorophenyliododifluoride, $\mathrm{C}_{6} \mathrm{~F}_{5} \mathrm{IF}_{2}$, has been investigated by single crystal X-ray crystallography and by multinuclear NMR, IR and Raman spectroscopy. ${ }^{180}$ The X-ray crystal and molecular structures of $p$-(difluoroiodo)toluene and $m$-(difluoroiodo)nitrobenzene have been reported in a Ph.D. dissertation in $1996 .{ }^{192}$

3.2.3. Reactions-(Difluoro)iodoarenes are powerful and selective fluorinating reagents towards various organic substrates. Various $\beta$-dicarbonyl compounds can be selectively fluorinated at the $\alpha$-position by 4 -(difluoroiodo)toluene and HF-amine complex. ${ }^{193}$ This fluorination can also be performed electrochemically using 4-(difluoroiodo)toluene generated in situ from iodotoluene in $\mathrm{Et}_{3} \mathrm{~N}-5 \mathrm{HF}$ in an undivided cell under constant potential. ${ }^{187}$ More recently, Hara and co-workers have reported a modified procedure that allows to prepare monofluorinated products $\mathbf{4 7}$ from $\beta$-ketoesters, $\beta$-ketoamides and $\beta$-diketones $\mathbf{4 6}$ in good yields under mild conditions without the addition of the HF-amine complexes (Scheme 14). 194 Ketones cannot be directly fluorinated by (difluoro)iodoarenes; however, $\alpha$-fluoroketones 
can be prepared by the reaction of silyl enol ethers with 4-(difluoroiodo)toluene in the presence of $\mathrm{BF}_{3} \cdot \mathrm{OEt}_{2}$ and the $\mathrm{Et}_{3} \mathrm{~N}-\mathrm{HF}$ complex. ${ }^{195}$

Treatment of $\alpha$-phenylthio esters 48 with one equivalent of 4 -(difluoroiodo)toluene affords the $\alpha$-fluoro sulfides $\mathbf{4 9}$ in good overall yield through a fluoro-Pummerer reaction (Scheme 15).

196 Addition of a second equivalent of 4-(difluoroiodo)toluene in this reaction produced $\alpha, \alpha$ difluoro sulfides and a third led to $\alpha, \alpha$-difluoro sulfoxides. This sequential fluorinationoxidation behavior was exploited in the one-pot synthesis of 3-fluoro-2 $(5 H)$-furanone starting from (3R)-3-fluorodihydro-2(3H)-furanone. ${ }^{196}$ The $\alpha$-monofluorination of sulfanyl amides can be achieved by treatment of $\alpha$-phenylsulfanylacetamides with one equivalent of 4(difluoroiodo)toluene under similar conditions. ${ }^{197}$

Arrica and Wirth have reported the monofluorination of a series of $\alpha$-acceptor-substituted selenides 50 using (difluoroiodo)toluene (Scheme 16). ${ }^{189}$ Although the yields of products 51 are only moderate, the reactions are usually very clean and, under the reaction conditions used, no further oxidized products are observed.

Fluorinated five- to seven-membered cyclic ethers 55-57 were stereoselectively synthesized from iodoalkyl substituted four- to six-membered cyclic ethers $\mathbf{5 2 - 5 4}$ by fluorinative ringexpansion reaction using (difluoroiodo)toluene (Scheme 17). ${ }^{198}$

Furrow and Myers have developed a convenient general procedure for the esterification of carboxylic acids with diazoalkanes $\mathbf{5 9}$ generated in situ by the oxidation of $N$-tertbutyldimethylsilylhydrazones $\mathbf{5 8}$ with (difluoroiodo)benzene (Scheme 18). ${ }^{199}$ This protocol affords various esters 60 from a broad range of carboxylic acids and, compared to the traditional esterification using diazoalkanes, offers significant advantages with regard to safety, because the diazo intermediates $\mathbf{5 9}$ are neither isolated nor achieve appreciable concentrations during the reaction.

4-(Difluoroiodo)toluene reacts with terminal alkenes $\mathbf{6 1}$ to give vic-difluoroalkanes $\mathbf{6 2}$ in moderate yields (Scheme 19). ${ }^{200}$ The cyclohexene derivative 63 reacts with this reagent under similar conditions with the stereoselective formation of $c i s$-difluoride $64 .^{200}$ The observed syn-stereoselectivity of this difluorination is explained by a two-step mechanism involving the anti-addition of the reagent to the double bond through a cyclic iodonium intermediate at the first step, and then nucleophilic substitution of iodotoluene with fluoride anion in the second step. The reaction of substituted cyclic alkenes 65 with 4 -(difluoroiodo)toluene and $\mathrm{Et}_{3} \mathrm{~N}-5 \mathrm{HF}$ results in a fluorinating ring-contraction with the selective formation of difluoroalkyl substituted cycloalkanes $\mathbf{6 6}$ (Scheme 19). ${ }^{201}$

The fluorination of alkenes 67, 69 and alkynes 71 with 4-(difluoroiodo)toluene in the presence of iodine affords vic-fluoroiodoalkanes 68, 70 and fluoroiodoalkenes 72 in moderate to good yields (Scheme 20). ${ }^{202}$ This reaction proceeds in a Markovnikov fashion and with prevalent anti-stereoselectivity via the initial addition of the electrophilic iodine species followed by nucleophilic attack of fluorine anion. The analogous reaction of alkenes and alkynes with 4(difluoroiodo)toluene in the presence of diphenyl diselenides affords the respective products of phenylselenofluorination in good yields. ${ }^{203}$

The reaction of 4-(difluoroiodo)toluene with 5-halopentynes with a four-, five-, or sixmembered carbocycle $\mathbf{7 3}$ afforded the ring-expanded $(E)$ - $\delta$-fluoro- $\beta$-halovinyl iodonium tetrafluoroborates $\mathbf{7 4}$ stereoselectively in high yields (Scheme 21). ${ }^{204}$ This reaction proceeds via a sequence of $\lambda^{3}$-iodanation-1,4-halogen shift-ring enlargement-fluorination steps.

4-(Difluoroiodo)toluene and other (difluoroiodo)arenes are commonly employed as reagents for the preparation of iodonium salts (see also Section 3.9) ${ }^{205-208}$ Especially useful is the 
reaction of potassium organotrifluoroborates with 4-(difluoroiodo)toluene affording various iodonium tetrafluoroborate salts under mild conditions. ${ }^{205}$

\subsection{Chlorides}

3.3.1. Preparation-The most general approach to (dichloroiodo)arenes involves the direct chlorination of iodoarenes with chlorine in a suitable solvent, such as chloroform or dichloromethane. ${ }^{209}$ This method can be applied to the large scale (20-25 kg) preparation of $\mathrm{PhICl}_{2}$ by the reaction of iodobenzene with chlorine at -3 to $+4{ }^{\circ} \mathrm{C}$ in dichloromethane. ${ }^{210}$ The direct chlorination of iodoarenes $\mathbf{7 5}$ and $\mathbf{7 7}$ has recently been used for the preparation of 4,4'bis(dichloroiodo)biphenyl 76 and 3-(dichloroiodo)benzoic acid 78 (Scheme 22), which are convenient recyclable hypervalent iodine reagents. ${ }^{211}$

In order to avoid the use of elemental chlorine, the chlorination of iodoarenes can be effected in situ in aqueous hydrochloric acid in the presence of an appropriate oxidant, such as $\mathrm{KMnO}_{4}$, activated $\mathrm{MnO}_{2}, \mathrm{KClO}_{3}, \mathrm{NaIO}_{3}$, concentrated $\mathrm{HNO}_{3}, \mathrm{NaBO}_{3}, \mathrm{Na}_{2} \mathrm{CO}_{3} \cdot \mathrm{H}_{2} \mathrm{O}_{2}$, $\mathrm{Na}_{2} \mathrm{~S}_{2} \mathrm{O}_{8}, \mathrm{CrO}_{3}$, and the urea- $\mathrm{H}_{2} \mathrm{O}_{2}$ complex. ${ }^{212-214}$ For example, the chlorination of iodoarenes in a biphasic mixture of carbon tetrachloride and concentrated hydrochloric acid in the presence of $\mathrm{Na}_{2} \mathrm{~S}_{2} \mathrm{O}_{8}$ affords the corresponding (dichloroiodo)arenes in $60-100 \%$ crude yields. ${ }^{213} \mathrm{~A}$ recently reported convenient and mild approach to (dichloroiodo)arenes $\mathbf{8 0}$ consists of the chlorination of iodoarenes $\mathbf{7 9}$ using concentrated hydrochloric acid and aqueous sodium hypochlorite (Scheme 23). ${ }^{215}$ Sodium chlorite, $\mathrm{NaClO}_{2}$, can also be used in this procedure; however, in this case the chlorination takes longer time (3 hours at room temperature) and the yields of products $\mathbf{8 0}$ are generally lower. ${ }^{215}$

The other synthetic approaches to (dichloroiodo)arenes are represented by the one-pot oxidative iodination/chlorination of arenes with iodine and the appropriate oxidant in hydrochloric acid ${ }^{216}$ and by the treatment of iodosylbenzene with trimethylsilyl chloride. ${ }^{217}$, 218

(Dichloroiodo)arenes are generally isolated as light and heat sensitive yellow crystalline solids, which are insufficiently stable for extended storage even at low temperatures.

3.3.2. Structural Studies-Several X-ray crystallographic studies of organoiododichlorides, $\mathrm{RICl}_{2}$, have been reported in the literature. The first X-ray crystal structures of $\mathrm{PhICl}_{2}{ }^{219}$ and $4-\mathrm{ClC}_{6} \mathrm{H}_{4} \mathrm{ICl}_{2}{ }^{220}$ published in 1953 and 1956 were imprecise by modern standards. More recently, a good quality structure of $\mathrm{PhICl}_{2}$ obtained at low temperature has been reported. ${ }^{221}$ The molecule of $\mathrm{PhICl}_{2}$ has the characteristic T-shape with primary I-Cl bond distances of $2.47 \AA$ and $2.49 \AA$, and $\mathrm{Cl}-\mathrm{I}-\mathrm{C}$ bond angles of 87.8 and $89.2^{\circ}$. In the solid state the molecules form an infinite zig-zagged chain, in which one of the chlorine atoms interacts with the iodine of the next unit with an intermolecular $\mathrm{I} \cdot{ }^{\circ} \mathrm{Cl}$ secondary bond distance of $3.42 \AA$. The coordination of iodine is distorted square planar with the lone pairs occupying the trans-positions of a pseudooctahedron. ${ }^{221}$

$\mathrm{X}$-ray structures of two sterically encumbered (dichloroiodo)arenes, 2,4,6- $\mathrm{Pr}_{3}{ }_{3} \mathrm{C}_{6} \mathrm{H}_{2} \mathrm{ICl}_{2}{ }^{222}$ and $\mathrm{ArICl}_{2}[\mathrm{Ar}=2,6 \text {-bis(3,5-dichloro-2,4,6-trimethylphenyl)benzene }]^{223}$ have been reported. Both molecules have the expected T-shaped geometry; the latter molecule has $\mathrm{Cl}-\mathrm{I}-\mathrm{C}$ angles

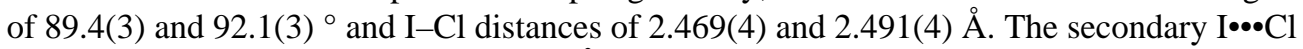
bond distance in this compound is $3.816 \AA$, which indicates a significant reduction of intermolecular association as compared to $\mathrm{PhICl}_{2} \cdot{ }^{223}$ The recently reported X-ray crystal structure of $o$-nitrobenzeneiododichloride, $2-\mathrm{NO}_{2} \mathrm{C}_{6} \mathrm{H}_{4} \mathrm{ICl}_{2}$, does not show any significant intramolecular interaction between the iodine(III) center and the oxygen atom of the nitro group in the ortho position ( $[\cdots 0 \mathrm{O}$ bond distance $3.0 \AA) .{ }^{99}$ 
$\mathrm{X}$-ray structure of the $\mathrm{PhICl}_{2}$ adduct with tetraphenylphosphonium chloride,

$\left[\mathrm{Ph}_{4} \mathrm{P}\right]^{+}\left[\mathrm{PhICl}_{3}\right]^{-}$, has been reported. ${ }^{224}$ The $\left[\mathrm{PhICl}_{3}\right]^{-}$anions in this structure have a planar coordination environment at the iodine atom. The $\mathrm{I}-\mathrm{Cl}$ bond length of the chlorine atom

trans to the $\mathrm{Ph}$ group is much longer (3.019 $\AA$ ) than the bond distance to the $c i s \mathrm{Cl}$ atoms ( 2.504 A). ${ }^{224}$

X-ray crystal structures of two perfluoroalkyliododichlorides, $\mathrm{CF}_{3} \mathrm{CH}_{2} \mathrm{ICl}_{2}$ and $\mathrm{CHF}_{2}\left(\mathrm{CF}_{2}\right)_{5} \mathrm{CH}_{2} \mathrm{ICl}_{2}$, have been reported. ${ }^{225} \mathrm{In}$ comparison to $\mathrm{PhICl}_{2}$, which has a simple chain structure, perfluoroalkyliododichlorides have more complicated structures in which weak interactions between chains, coupled with aggregation of perfluoro groups, result in the formation of layers.

3.3.3. Reactions-(Dichloroiodo)arenes have found practical application as reagents for chlorination or other oxidative transformations of various organic substrates. Chlorinations of alkanes with (dichloroiodo)arenes proceed via a radical mechanism and generally require photochemical conditions or the presence of radical initiators in solvents of low polarity, such as chloroform or carbon tetrachloride. ${ }^{5}$ The chlorination of alkenes may follow a radical or ionic mechanism depending on the conditions. ${ }^{211,226-228}$ For example, norbornene reacts with (dichloroiodo)benzene under radical conditions in nonpolar solvents with the formation of 1,2dichlorides as the only detectable products. ${ }^{226}$ In contrast, reactions of (dichloroiodo)benzene with various monoterpenes in methanol have an ionic mechanism and afford the respective products of chloromethoxylation of the double bond with high regio- and stereoselectivity. ${ }^{228}$ Likewise, the reaction of 4,4'-bis(dichloroiodo)biphenyl $\mathbf{7 6}$ with styrene derivatives 81 in methanol affords exclusively the products of electrophilic chloromethoxylation 82 (Scheme 24). ${ }^{211}$

(Dichloroiodo)arenes can also be used for the chlorination of electron-rich aromatic compounds. Aminoacetophenone $\mathbf{8 3}$ is selectively chlorinated with (dichloroiodo)benzene to give product 84 in good yield (Scheme 25). This process can be scaled up to afford $24.8 \mathrm{~kg}$ of product 84 with $94 \%$ purity. ${ }^{210}$

(Dichloroiodo)toluene was found to be a suitable chlorinating agent in the catalytic asymmetric chlorination of $\beta$-keto esters 85 , catalyzed by the titanium complex $\mathbf{8 6}$, leading to the respective $\alpha$-chlorinated products $\mathbf{8 7}$ in moderate to good yields and enantioselectivities (Scheme 26).

The enantioselectivity of this reaction showed a remarkable temperature dependence, and the maximum selectivity was obtained at $50{ }^{\circ} \mathrm{C} .229$

The reaction of $N$-protected pyrrolidine $\mathbf{8 8}$ with 4-nitrobenzeneiododichloride affords $\alpha$ hydroxy- $\beta, \beta$-dichloropyrrolidine $\mathbf{8 9}$ as the main product (Scheme 27) via a complex ionic mechanism involving a triple $\mathrm{C}-\mathrm{H}$ bond activation. This oxidative pathway has been demonstrated to be general for several saturated, urethane protected nitrogen heterocyclic systems. ${ }^{218}$

Treatment of 5,10,15-trisubstituted porphyrins 90 with (dichloroiodo)benzene affords the corresponding meso-chlorinated porphyrins 91 (Scheme 28). ${ }^{230}$ The reactions of trisubstituted $\mathrm{Zn}$-porphyrins lead to the products of coupling, meso, meso-linked bisporphyrins, along with the meso-chlorinated products. The chlorination of 5,10,15,20-tetraarylporphyrins, in which all meso-positions are substituted, under similar conditions affords $\beta$-monochlorinated products in high yields. ${ }^{230}$

(Dichloroiodo)arenes have been applied in various oxidative transformations of organic substrates. An efficient and mild procedure has been described for the oxidation of different types of alcohols to carbonyl compounds using 2,2,6,6-tetramethylpiperidine-1-oxyl 
(TEMPO) as the catalyst and (dichloroiodo)benzene as a stoichiometric oxidant at $50{ }^{\circ} \mathrm{C}$ in chloroform solution in the presence of pyridine. ${ }^{215}$ Under these conditions 1,2-diols are oxidized to $\alpha$-hydroxy ketones or $\alpha$-diketones depending upon the amount of $\mathrm{PhICl}_{2}$ used. A competitive study has shown that this system preferentially oxidizes aliphatic secondary alcohols over aliphatic primary alcohols. ${ }^{215}$

A simple and mild system using bis(dichloroiodo)biphenyl $\mathbf{7 6}$ in combination with tetraethylammonium bromide at room temperature has been developed for selective debenzylation of sugars. Acetates, benzoate, and sensitive glycosidic linkages are unaffected under the reaction conditions. A specific example of the debenzylation of benzyl 4-O-benzoyl 2,3-O-isopropylidene- $\alpha$-L-arabinopyranoside $\mathbf{9 2}$ is shown in Scheme 29. ${ }^{231}$

An efficient route to the 3-iodo-4-aryloxypyridinones 95, which are highly potent nonnucleoside inhibitors of HIV-1 reverse transcriptase, has been developed starting from 4hydroxy substituted pyridinone 93 and (dichloroiodo)arenes 94 (Scheme 30). ${ }^{232,233}$

Various organic substrates, such as enol silyl ethers, ketene silyl acetals, $\beta$-dicarbonyl compounds, ${ }^{234}$ alkynes, ${ }^{235}$ and para-unsubstituted phenols and naphthols, ${ }^{236}$ can be effectively thiocyanated with the combination reagent $\mathrm{PhICl}_{2} / \mathrm{Pb}(\mathrm{SCN})_{2}$. More recently, Prakash and co-workers have reported an improved method for the thiocyanation of 2arylindan-1,3-diones, phenols, and anilines using a reagent combination of (dichloroiodo) benzene and potassium thiocyanate in dry dichloromethane. ${ }^{237}$ For example, the paraunsubstituted phenols and anilines $\mathbf{9 6}$ are efficiently converted under these reaction conditions to the respective $p$-thiocyanato derivatives $\mathbf{9 7}$ in high yields (Scheme 31).

Very recently, Zhang and co-workers have reported the application of (dichloroiodo)benzene in combination with sodium azide for the effective synthesis of carbamoyl azides from aldehydes. ${ }^{238}$

(Dichloroiodo)benzene is commonly used as a reagent for the oxidation or chlorination of various transition metal complexes. Recent examples include the oxidation of $\mathrm{d} 8 \bullet \bullet \mathrm{d} 10$ heterobimetallic $\mathrm{Pt}(\mathrm{II})-\mathrm{Au}$ (I) complex to give the $\mathrm{d} 7$-d9 Pt(III)-Au(II) complex containing a $\mathrm{Pt}(\mathrm{III})-\mathrm{Au}$ (II) bond, ${ }^{239}$ and oxidations or chlorinations of palladium, ${ }^{240,241}$ cobalt, ${ }^{242}$ vanadium, ${ }^{243}$ and molybdenum ${ }^{244}$ complexes. Several examples of Pd-catalyzed chlorinations of organic substrates using (dichloroiodo)benzene have also been reported. ${ }^{245,246}$

\section{4. [Bis(acyloxy)iodo]arenes}

[Bis(acyloxy)iodo]arenes, $\operatorname{ArI}\left(\mathrm{O}_{2} \mathrm{CR}\right)_{2}$, are the most important, well investigated, and practically useful organic derivatives of iodine(III). Two of them, (diacetoxyiodo)benzene, commonly abbreviated as DIB, PID, PIDA (phenyliodine diacetate), IBD, or IBDA (iodosobenzene diacetate) and [bis(trifluoroacetoxy)iodo]benzene, abbreviated as BTI or PIFA [(phenyliodine bis(trifluoroacetate)], are commercially available and widely used oxidizing reagents. In this review, the abbreviations DIB and BTI, originally suggested by Varvoglis, ${ }^{2}$ will be used. Over a thousand research papers dealing mainly with various synthetic applications of DIB and BTI have been published since the year of 2000. The use of [bis (acyloxy)iodo]arenes as precursors to other iodine(III) compounds and as the reagents for oxidation of alkynes, allenes, alkenes, enolizable ketones, electron-rich aromatic compounds, alcohols, organic derivatives of nitrogen, phosphorus, sulfur, selenium, tellurium, and other organic substrates has been discussed in previous reviews. ${ }^{2,5,6}$ In this section, the preparation, structural studies, and typical recent examples of synthetic applications of [bis(acyloxy)iodo] arenes are overviewed. 
3.4.1. Preparation-Two general approaches are used for the preparation of [bis(acyloxy) iodo]arenes: (1) the oxidation of iodoarenes in the presence of a carboxylic acid, and (2) a ligand exchange reaction of the readily available DIB with an appropriate carboxylic acid. The most common and practically important representative of [bis(acyloxy)iodo]arenes, DIB, is usually prepared by the oxidation of iodobenzene with peracetic acid in acetic acid. ${ }^{247} \mathrm{~A}$ similar peracid oxidation of substituted iodobenzenes can be used for the preparation of other [bis (acyloxy)iodo]arenes. In particular, the polymer-supported analogs of DIB have been prepared by treatment of poly(iodostyrene) or aminomethylated poly(iodostyrene) with peracetic acid, $30,248-250$ and the ion-supported [bis(acyloxy)iodo]arenes, imidazolium derivatives 98 and 99, have been prepared by the peracetic oxidation of the appropriate aryliodides. ${ }^{251,252}$ Likewise, various [bis(trifluoroacetoxy)iodo]arenes can be synthesized in high yield by the oxidation of the respective iodoarenes with peroxytrifluoroacetic acid in trifluoroacetic acid. 253-255
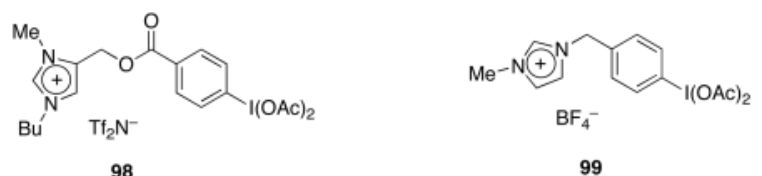

A modification of this method consists of the oxidative diacetoxylation of iodoarenes in acetic or trifluoroacetic acid using appropriate oxidants, such as periodates, ${ }^{256-258}$ sodium percarbonate ${ }^{259} \mathrm{~m}$-chloroperoxybenzoic acid, ${ }^{260-264}$ potassium peroxodisulfate, ${ }^{265,266}$ $\mathrm{H}_{2} \mathrm{O}_{2}$-urea, ${ }^{267}$ Selectfluor, ${ }^{183}$ and sodium perborate. ${ }^{264,268-274}$ The oxidation of iodoarenes with sodium perborate in acetic acid at $40{ }^{\circ} \mathrm{C}$ is the most simple and general procedure that has been used for a small scale preparation of numerous (diacetoxyiodo)-substituted arenes and hetarenes. ${ }^{264,268-274}$ This method can be improved by performing the perborate oxidation in the presence of trifluoromethanesulfonic acid. ${ }^{275} \mathrm{~A}$ further convenient modification of this approach employs the interaction of arenes $\mathbf{1 0 0}$ with iodine and potassium peroxodisulfate in acetic acid (Scheme 32). ${ }^{276}$ The mechanism of this reaction probably includes the oxidative iodination of arenes, followed by diacetoxylation of ArI in situ leading to (diacetoxyiodo) arenes 101.

The second general approach to [bis(acyloxy)iodo]arenes is based on the ligand exchange reaction of a (diacetoxyiodo)arene (usually DIB) with the appropriate carboxylic acid. A typical procedure consists of heating DIB with a non-volatile carboxylic acid $\mathrm{RCO}_{2} \mathrm{H}$ in the presence of a high boiling solvent, such as chlorobenzene (Scheme 33). ${ }^{277-282}$ The equilibrium in this reversible reaction can be shifted towards the synthesis of the product 102 by distillation under reduced pressure of the relatively volatile acetic acid formed during the reaction. This procedure, in particular, has recently been used for the preparation of the glutamate-derived diacyloxyiodobenzenes 103,278 protected amino acid derivatives 104,280 the cinnamate derivative $\mathbf{1 0 5}, 282$ and 3-methylfurazan-4-carboxylic acid derivative $\mathbf{1 0 6} .^{283}$

The reactions of DIB with stronger carboxylic acids usually proceed under milder conditions at room temperature. A convenient procedure for the preparation of BTI consists of simply dissolving DIB in trifluoroacetic acid and evaporating to a small volume. ${ }^{284} \mathrm{In}$ a related method, used for the preparation of a series of $\mathrm{PhI}\left(\mathrm{OCOCO}_{2} \mathrm{R}\right)_{2}$, DIB is treated with oxalyl chloride in the respective alcohol, $\mathrm{ROH} .{ }^{285}$

[Bis(acyloxy)iodo]arenes are generally colorless, stable microcrystalline solids, which can be easily recrystallized and stored for extended periods of time without significant decomposition.

3.4.2. Structural Studies-Numerous structural reports on [bis(acyloxy)iodo]arenes were summarized in earlier reviews. ${ }^{2,5,6}$ In general, single crystal X-ray structural data for [bis 
(acyloxy)iodo]benzenes indicate a pentagonal planar coordination of iodine within the molecule, combining the primary T-shaped iodine(III) geometry with two secondary intramolecular I $\bullet \bullet O$ interactions with the carboxylate oxygens. ${ }^{286} \mathrm{X}$-ray crystal structures of four new compounds, 1,3,5,7-tetrakis[4-(diacetoxyiodo)phenyl]adamantane 107,260 tetrakis [4-(diacetoxyiodo)phenyl]methane 108, 261 3-[bis(trifluoroacetoxy)iodo]benzoic acid 109,103 and 1-(diacetoxyiodo)-2-nitrobenzene $\mathbf{1 1 0}, 99$ have been reported in the recent literature.
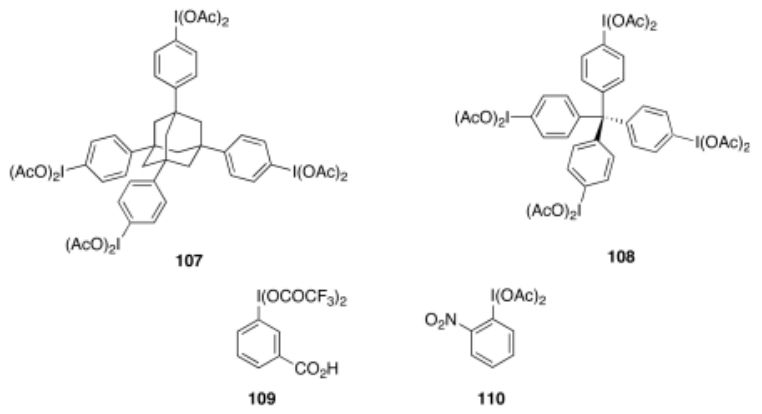

In the molecule of trifluoroacetate 109 , the $\mathrm{C}-\mathrm{I}$ bond length is $2.083 \AA$, the primary $\mathrm{I}-\mathrm{O}$ bond lengths are 2.149 and $2.186 \AA$, and the intramolecular secondary $\mathrm{I} \cdot \bullet \cdot \mathrm{O}$ interactions with the carboxylate oxygens have distances of $\mathrm{I}(1) \bullet \bullet \bullet \mathrm{O}(5) 3.146 \AA$ and $\mathrm{I}(1) \bullet \bullet \bullet \mathrm{O}(4) 3.030 \AA$; these five intramolecular interactions result in the pentagonal planar coordination of iodine within the molecule. ${ }^{103}$ In addition to the five intramolecular interactions, an intermolecular coordination of iodine atom to one the carboxylic oxygens of the neighboring molecule is also present with a distance of $3.023 \AA$. It is interesting to note that the presence of the meta-carboxylic group does not have any noticeable effect on the molecular geometry of compound 109, which is very similar to the X-ray crystal structure of [bis(trifluoroacetoxy)iodo]benzene. ${ }^{286}$ The X-ray crystal structure of 1-(diacetoxyiodo)-2-nitrobenzene $\mathbf{1 1 0}$ does not show any significant intramolecular interaction between the iodine(III) center and the oxygen atom of the nitro group

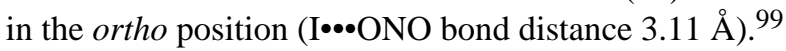

The ${ }^{17} \mathrm{O}$ NMR study of bis(acyloxy)iodoarenes in chloroform has confirmed that the T-shaped structure of iodine(III) compounds observed in the solid state is also adopted in solution. ${ }^{109 \text {, }}$ 110 The carboxylic groups of bis(acyloxy)iodoarenes show a dynamic behavior, which is explained by a $\left.{ }^{1,3}\right]$ sigmatropic shift of the iodine atom between the two oxygen atoms of the carboxylic groups. ${ }^{110}$

3.4.3. Oxidation of Alcohols-An efficient procedure for the oxidation alcohols with DIB in the presence of catalytic amounts of TEMPO (2,2,6,6-tetramethylpiperidin-1-oxyl), originally developed by Piancatelli, Margarita and co-workers, ${ }^{287}$ has been frequently used in recent years. ${ }^{264,288-293}$ An optimized protocol, published in Organic Synthesis for the oxidation of nerol 111 to nepal 112 (Scheme 34), consists of the treatment of the alcohol 111 solution in buffered $(\mathrm{pH} 7)$ aqueous acetonitrile with DIB and TEMPO ( 0.1 equivalent $)$ at $0^{\circ}$ C for 20 minutes. ${ }^{288}$

This procedure exhibits a very high degree of selectivity for the oxidation of primary alcohols to aldehydes, without any noticeable overoxidation to carboxylic acids, and a high chemoselectivity in the presence of either secondary alcohols or of other oxidizable moieties. 287 A similar oxidation procedure has been used for the oxidation of (fluoroalkyl)alkanols, $\mathrm{R}_{\mathrm{F}}\left(\mathrm{CH}_{2}\right)_{\mathrm{n}} \mathrm{CH}_{2} \mathrm{OH}$, to the respective aldehydes, ${ }^{289}$ in the one-pot selective oxidation/ olefination of primary alcohols using DIB-TEMPO system and stabilized phosphorus ylides, 290 and in the chemo-enzymatic oxidation-hydrocyanation of $\gamma, \delta$-unsaturated alcohols. ${ }^{291}$ Other [bis(acyloxy)iodo]arenes can be used instead of DIB in the TEMPO catalyzed 
oxidations, such as the recyclable monomeric 1,3,5,7-tetrakis[4-(diacetoxyiodo)phenyl] adamantane $1 \mathbf{1 0 7}^{260}$ and biphenyl- and terphenyl-based (diacetoxyiodo)arenes, ${ }^{264}$ and the polymer-supported DIB. ${ }^{292,293}$ Further modifications of this method include the use of polymer-supported TEMPO, ${ }^{294}$ fluorous-tagged TEMPO, ${ }^{295,296}$ ion-supported TEMPO, 297 and TEMPO immobilized on silica. ${ }^{291}$

Based on the ability of the DIB-TEMPO system to selectively oxidize primary alcohols to the corresponding aldehydes in the presence of secondary alcohols, Forsyth and co-workers have developed selective oxidative conversion of a variety of highly functionalized $1^{\circ}, 2^{\circ}-1,5$-diols into the corresponding $\delta$-lactones. ${ }^{298}$ A representative example of converting substrate 113 to the $\delta$-lactone 114 is shown in Scheme 35. Monitoring of this reaction showed the initial formation of the intermediate lactol species, which then undergoes further oxidation to the lactone. ${ }^{298}$ A similar DIB-TEMPO promoted $\gamma$-lactonization has recently been utilized in the asymmetric total synthesis of the antitumor (+)-eremantholide A. ${ }^{299}$

[Bis(acyloxy)iodo] arenes in the presence of $\mathrm{KBr}$ in water can oxidize primary and secondary alcohols analogously to the $\mathrm{PhIO} / \mathrm{KBr}$ system. ${ }^{124}$ The oxidation of primary alcohols affords carboxylic acids or esters, ${ }^{123,300}$ while the oxidation of secondary alcohols under similar conditions results in the formation of the respective ketones in excellent yields. ${ }^{261}$ In a specific example, primary alcohols 115 are readily oxidized to methyl esters 116 upon treatment with polystyrene-supported $\mathrm{DIB}$ in the presence of $\mathrm{KBr}$ in the acidic aqueous methanol solution (Scheme 36). ${ }^{300}$ Aldehydes can be converted to methyl esters by a similar procedure using DIB and $\mathrm{NaBr}{ }^{301}$

The oxidation of various primary and secondary alcohols with the ion-supported [bis(acyloxy) iodo]arene 99 (1.4 equivalents) in the ionic liquid [emim $]^{+}\left[\mathrm{BF}_{4}\right]^{-}$(1-ethyl-3-

methylimidazolium tetrafluoroborate) in the presence of bromide anion selectively affords the respective carbonyl compounds without overoxidation to carboxylic acids. ${ }^{251}$

Molecular iodine can serve as an efficient catalyst in the oxidation of secondary alcohols to ketones and primary alcohols to carboxylic acids using DIB as an oxidant in acetonitrile solution. ${ }^{302}$ The oxidation of primary alcohols or aldehydes with the DIB/I 2 system in methanol solution affords the respective methyl esters in excellent yields. ${ }^{303}$

Only a few examples of uncatalyzed oxidation of alcohols with [bis(acyloxy)iodo]arenes have been reported. ${ }^{249,304,305}$ Substituted benzyl alcohols can be oxidized by BTI in aqueous acetic acid to the corresponding benzaldehydes. ${ }^{304}$ Vicinal fullerene diol is oxidized to fullerene dione in $80 \%$ yield by DIB in benzene at $35{ }^{\circ} \mathrm{C} .{ }^{305}$ Various vicinal diols 117 (13 examples) can be oxidized to aldehydes 118 using polymer-supported DIB (Scheme 37). ${ }^{249}$ Protecting groups such as $\mathrm{OAc}, \mathrm{OR}, \mathrm{OBn}, \mathrm{OBz}$, and isopropylidene in the substrates are stable under these reaction conditions. cis-1,2-Cyclohexandiol is converted to 1,6-hexandial in this reaction. 249

\subsubsection{Oxidative Functionalization of Carbonyl Derivatives and Unsaturated} Compounds-In the 1980s Moriarty and co-workers have developed a particularly useful methodology for the oxidative $\alpha$-functionalization of enolizable carbonyl compounds or their enol ethers using DIB or other hypervalent iodine oxidants. ${ }^{306-309}$ The applications of this methodology in organic synthesis, especially in the chemistry of heterocyclic compounds, have been summarized in several reviews. ${ }^{9,37,40,310}$ Ochiai and co-workers have recently reported a catalytic variant of $\alpha$-acetoxylation of ketones based on the in situ generation of DIB from iodobenzene using $m$-chloroperbenzoic acid $(m \mathrm{CPBA})$ as a terminal oxidant ${ }^{311}$ In a typical example, the oxidation of a ketone with $m \mathrm{CPBA}$ ( 2 equiv.) in acetic acid in the presence of a catalytic amount of $\mathrm{PhI}\left(0.1\right.$ equiv.), $\mathrm{BF}_{3} \bullet \mathrm{OEt}_{2}$ ( 3 equiv.) and water ( 5 equiv.) at room 
temperature under argon affords the respective $\alpha$-acetoxy ketone in $63-84 \%$ isolated yield. $p$ Methyl- and $p$-chloroiodobenzene can also serve as efficient catalysts in the $\alpha$-acetoxylation of ketones using $m \mathrm{CPBA}$ as a terminal oxidant. ${ }^{311}$

The oxidative functionalization of silyl enol ethers 119 with DIB as oxidant and $\mathrm{N}$ aminophthalimide $\mathbf{1 2 0}$ as external nucleophile has recently been employed in the stereoselective synthesis of trans- $\alpha$-ketohydrazones $\mathbf{1 2 1}$ in good yields under mild conditions (Scheme 38). ${ }^{312}$ The mechanism of this reaction involves the initial formation of $\alpha$ ketohydrazines, which are further oxidized by DIB to give the final ketohydrazones $\mathbf{1 2 1}$.

Numerous recent examples of oxidative transformations of alkenes using [bis(acyloxy)iodo] arenes have been reported. ${ }^{138,282,313-318}$ [Bis(trifluoroacetoxy)iodo]benzene reacts with alkenes in the absence of any additive or catalyst affording bis(trifluoroacetates), which can be converted into the corresponding diols or carbonyl compounds by hydrolysis. ${ }^{313,319}$ For example, cyclohexene reacts with BTI in dichloromethane under reflux conditions to give cis-1,2-bis(trifluoroacetate) $\mathbf{1 2 2}$ in almost quantitative yield (Scheme 39). In the case of bicyclic alkenes, such as norbornene or benzonorbornadiene 123, the rearranged products (e.g. 124) are predominantly formed. ${ }^{313}$ Similar rearranged products are formed in the reactions of alkenes with DIB in the presence of strong acids. ${ }^{314}$

[Bis(acyloxy)iodo]arenes can be used as the oxidants in organocatalytic, asymmetric epoxidation of $\alpha, \beta$-unsaturated aldehydes using imidazolidinone catalyst $\mathbf{1 2 6}{ }^{138}$ In a specific example, the reaction of aldehyde $\mathbf{1 2 5}$ with DIB affords epoxide 127 with good enantioselectivity (Scheme 40).

A procedure for the preparation of aromatic aldehydes $\mathbf{1 2 9}$ from isopropenylbenzenes $\mathbf{1 2 8}$ and zeolite-supported DIB under microwave irradiation (Scheme 41) has been reported. This method was used for a clean and reproducible preparation of piperonal, vanillin and $p$ anisaldehyde in generally high yields and selectivities. ${ }^{315}$

In the 1990s, Tingoli and co-workers have found a general approach to various arylselenated products by the reaction of unsaturated compounds with diaryl diselenides and DIB. ${ }^{320-323}$ Several further modifications of this reaction have recently been reported. ${ }^{282,316-318}$ The reaction of gem-aryl-disubstituted methylenecyclopropanes with diphenyl diselenide and DIB produced the corresponding bis-phenylselenated rearranged products in moderate yields under mild conditions. ${ }^{318}$ A multicomponent reaction of allenes 130, diaryl diselenides, DIB, and alcohols or acids affords 3-functionalized-2-arylselenyl substituted allyl derivatives $\mathbf{1 3 1}$ in moderate yields (Scheme 42). ${ }^{316}$

Nifantiev and co-workers reported an improved preparative method for homogeneous azidophenylselenylation of glycols by the reaction with DIB, diphenyldiselenide, and trimethylsilyl azide. In a representative example, the reaction of tri- $O$-benzyl-galactal 132 with $\mathrm{DIB} / \mathrm{Ph}_{2} \mathrm{Se}_{2} / \mathrm{TMSN}_{3}$ in dichloromethane under mild conditions affords the corresponding selenoglycoside $\mathbf{1 3 3}$ in moderate yield (Scheme 43). ${ }^{317}$ The noncarbohydrate alkenes, such as styrene and substituted cyclopentenes, can also be azidophenylselenated under these conditions.

The selenodecarboxylation of cinnamic acid derivatives $\mathbf{1 3 4}$ with diaryldiselenides promoted by DIB in acetonitrile affords vinyl selenides $\mathbf{1 3 5}$ in moderate yields (Scheme 44). A similar reaction of arylpropiolic acids gives respective alkynyl selenides in $60-90 \%$ yields. ${ }^{282}$

Kirschning and co-workers have developed several experimental procedures for the stereoselective bromoacetoxylation or iodoacetoxylation of alkenes based on the interaction of DIB with iodide or bromide anions. ${ }^{324,325}$ The actual reacting electrophilic species in these 
reactions are the diacetylhalogen $(\mathrm{I})$ anions, $(\mathrm{AcO})_{2} \mathrm{I}^{-}$and $(\mathrm{AcO})_{2} \mathrm{Br}^{-}$, which can also be prepared as the polymer-supported variant. ${ }^{326-328}$ A similar iodocarboxylation of alkenes using amino acid-derived iodobenzene dicarboxylates 104 selectively affords the respective amino acid esters 136 in moderate yields (Scheme 45). ${ }^{280}$

Iodine in combination with [bis(acyloxy)iodo] arenes can be used for the oxidative iodination of aromatic and heteroaromatic compounds. ${ }^{6,329}$ A mixture of iodine and BTI in acetonitrile or methanol iodinates the aromatic ring of methoxy substituted alkyl aryl ketones to afford the products of electrophilic monoiodination in $68-86 \%$ yield. ${ }^{330}$ 1-Iodoalkynes can be prepared in good to excellent yields by the oxidative iodination of terminal alkynes with DIB, potassium iodide, and copper(I) iodide. ${ }^{331}$ A solvent-free, solid state oxidative halogenation of arenes using DIB as the oxidant has recently been reported. ${ }^{332}$ A recyclable reagent, [bis (trifluoroacetoxy)iodo]benzoic acid 109, can also be used as the oxidant in the oxidative iodination reactions. ${ }^{103,333}$ Substituted pyrazoles 137 can be iodinated to the corresponding 4-iodopyrazole derivatives $\mathbf{1 3 8}$ by treatment with iodine and DIB or polymer-supported DIB at room temperature (Scheme 46). ${ }^{334}$

Oxidative thiocyanation of the electron-rich aromatic compounds, including phenol ethers, dimethyl aniline, thiophene and $N$-methylindole, can be performed using ammonium thiocyanate and DIB as the oxidant at room temperature in acetonitrile solution. ${ }^{335}$ Likewise, the direct cyanation of a wide range of electron-rich heteroaromatic compound, such as pyrroles, thiophenes, and indoles, can be achieved under mild conditions using [bis(acyloxy) iodo]arenes and trimethylsilyl cyanide as the cyanide source. ${ }^{262,263}$ In a specific example, the $\mathrm{N}$-tosylpyrroles 139 are selectively cyanated at the 2-position using [bis(trifluoroacetoxy)iodo] benzene and trimethylsilyl cyanide to afford products 140 in good yields (Scheme 47). ${ }^{263}$

BTI in the presence of tert-butyl hydroperoxide can oxidize various aromatic hydrocarbons to afford the corresponding quinones. ${ }^{336}$ For example, naphthalene is oxidized to1,4naphthaquinone in a moderate yield upon treatment with BTI (1.5 equiv.) and tert-butyl hydroperoxide ( 5 equiv.) for 3 hours at $-30{ }^{\circ} \mathrm{C} .{ }^{336}$ The introduction of hydroxy, alkoxy and acetoxy groups to the activated aromatic ring using [bis(acyloxy)iodo] arenes as oxidants has also been reported. $N$-Arylamides can be hydroxylated in the para position by BTI in trifluoroacetic acid at room temperature. ${ }^{337}$ The oxidation of 2,5-dihydroxyacetophenone with DIB in different alcohols leads to a regioselective alkoxylation, providing a convenient route for the synthesis of 6-alkoxy-2,5-dihydroxyacetophenones. ${ }^{338}$ Likewise, the DIB-promoted oxidation of 6-hydroxyflavone and 6-hydroxyflavanones in acetic acid leads to regioselective acetoxylation affording the respective 5-acetoxylated products in 53-63\% yield. ${ }^{339}$

Applications of [bis(acyloxy)iodo]arenes in the oxidative transformations of phenolic compounds and in the biaryl coupling reaction will be discussed in Sections 3.4.6 and 3.4.7.

3.4.5. Oxidative Cationic Cyclizations, Rearrangements, and FragmentationsDIB and BTI are commonly used as the reagents in various cationic cyclizations, rearrangements, and fragmentations. ${ }^{6}$ The cyclizations, induced by hypervalent iodine reagents, are particularly useful in the synthesis of heterocycles. Tellitu and Domínguez have developed a series of BTI-promoted intramolecular amidation reactions, generalized in Scheme 48, leading to various five, six and seven-membered heterocycles $143 .{ }^{340-353}$ Experimental evidence supports the ionic mechanism of this reaction, involving $N$-acylnitrenium intermediates 142 generated in the initial reaction of the amide 141 with the hypervalent iodine reagent. ${ }^{340}$

This methodology with some variations (Scheme 48) has been utilized by Tellitu, Domínguez and co-workers in the synthesis of the following heterocyclic systems: heterocycle-fused 
quinolinone derivatives, ${ }^{341}$ 1,4-benzodiazepin-2-ones, ${ }^{342}$ benzo-, naphtho-, and heterocyclefused pyrrolo[2,1-c][1,4]diazepines, ${ }^{343} 2,3$-diarylbenzo[b]furans, ${ }^{344}$ quinolinone or pyrrolidinone derivatives, ${ }^{345}$ dibenzo[ $\left.a, c\right]$ phenanthridines, ${ }^{346}$ thiazolo-fused quinolinones, 347 isoindolinone and isoquinolin-2-one derivatives, ${ }^{348}$ indoline derivatives, ${ }^{349} 5$-aroylpyrrolidinones, ${ }^{350,351}$ and indazolone derivatives. ${ }^{352,353}$ Recent representative examples include the preparation of indoline derivatives 145 from anilides 144,349 pyrrolidinones 147 from alkynylamides 146,350,351 and indazol-3-ones 149 from anthranilamides 148 (Scheme 49). 352,353

Similar DIB or BTI induced cyclizations of the appropriate amide or amine precursors have been used in numerous useful synthetic transformations, such as: the synthesis of highly substituted pyrrolin-4-ones via BTI-mediated cyclization of enaminones, ${ }^{354}$ the synthesis of 2-substituted-4-bromopyrrolidines via DIB-induced intramolecular oxidative bromocyclization of homoallylic sulfonamides in the presence of $\mathrm{KBr},{ }^{355}$ the preparation of 2-( $N$-acylaminal $)$ substituted tetrahydropyrans by DIB-induced oxidative cyclization of hydroxy-substituted $N$-acyl enamines, ${ }^{356}$ the preparation of 1,2,4-thiadiazoles by the reaction of DIB or BTI with 1-monosubstituted thioureas, ${ }^{357,358}$ the synthesis of azaspirocyclic synthetic intermediates via the BTI-induced nitrenium ion cyclizations, ${ }^{359-365}$ the preparation of lactams and spiro-fused lactams from the reaction of $N$-acylaminophthalimides and BTI, 366 the stereocontrolled preparation of highly substituted lactams and $N$-hydroxy lactams from appropriate hydroxamates and BTI, ${ }^{365}$ the synthesis of 1,2,4-triazolo[4,3-a][1,8] naphthyridines using DIB-oxidation of 1,8-naphthyridin-2-ylhydrazones in the solid state, ${ }^{367}$ the synthesis of various substituted 1,2,4-triazolo[4,3-a]pyrimidines by the DIB-oxidation of the appropriate 2,4-pyrimidinylhydrazones, ${ }^{368-370}$ the preparation of thiazolo[2,3-c]-striazoles by the reaction of arenecarbaldehyde-4-arylthiazol-2-ylhydrazones with poly[(4diacetoxyiodo)styrene], ${ }^{371}$ the synthesis of pyrrolidino[60]fullerene from the DIB-promoted reaction between $\mathrm{C} 60$ and amino acid esters, ${ }^{372}$ 1,3,4-oxadiazoles from acylhydrazones by BTI oxidation, ${ }^{373-375}$ the synthesis of 1-aryl-4-methyl-1,2,4-triazolo[4,3-a]quinoxalines from arenecarboxaldehyde-3-methyl-2-quinoxalinylhydrazones, ${ }^{376,377}$ the synthesis of 1benzoyltetrahydroisoquinoline derivatives using polymer-supported BTI. ${ }^{378}$ Likewise, the preparation of benzopyrano- and furopyrano-2-isoxazoline derivatives from 2allyloxybenzaldoximes by DIB oxidation, ${ }^{379}$ the synthesis of various $\mathrm{N}$-substituted indole derivatives via BTI-mediated intramolecular cyclization of enamines, ${ }^{380}$ the synthesis of 2substituted benzothiazoles via the oxidative cyclization of thiobenzamides, ${ }^{381}$ the preparation of 2,3-diphenylquinoxaline-1-oxide from benzil- $\alpha$-arylimino oximes using DIB, ${ }^{382}$ the synthesis of 1-(5-aryl-[1,3,4]oxadiazol-2-ylmethyl)-3-(4-methoxyphenyl)-1H-[1,8] naphthyridin-2-ones by oxidative cyclization of [2-oxo-3-(4-methoxyphenyl)-2 $\mathrm{H}$ - $[1,8]$ naphthyridin-1-yl]acetic acid arylidenehydrazides with alumina-supported DIB under microwave irradiation, ${ }^{383}$ the synthesis of 2,5-disubstituted-1,3,4-oxadiazoles by via BTImediated oxidative cyclization of aldazines, ${ }^{384}$ the preparation of 2-substituted oxazolines from aldehydes and 2-amino alcohols using DIB as an oxidant, ${ }^{385}$ the synthesis of 3,4-bis(1phenyl-3-arylpyrazolyl)-1,2,5-oxadiazole- $N$-oxides by the DIB oxidation of pyrazole-4carboxaldehyde oximes, ${ }^{386}$ the synthesis of 2-arylbenzimidazoles from phenylenediamines and aldehydes via a one-step process using DIB as an oxidant, ${ }^{387}$ the DIB-mediated efficient synthesis of imidazoles from $\alpha$-hydroxy ketones, aldehydes and ammonium acetate, ${ }^{388}$ the preparation of dihydrooxazole derivatives by DIB-promoted 1,3-dipolar cycloaddition reactions of phthalhydrazide, ${ }^{389}$ and the synthesis of seco-psymberin/irciniastatin A via a DIBmediated cascade cyclization reaction. ${ }^{390}$ Very recently, Togo and Moroda have reported a DIB-mediated cyclization reaction of 2-aryl- $N$-methoxyethanesulfonamides using iodobenzene as a catalyst $(5-10 \mathrm{~mol} \%)$ and $m$-chloroperoxybenzoic acid as the stoichiometric oxidant. $^{391}$ 
Several examples of the DIB or BTI-induced cyclizations of non-amine substrates have also been reported. The DIB-mediated oxidative addition of 1,3-dicarbonyl compounds $\mathbf{1 5 0}$ to various alkenes 151 allows an efficient one-pot synthesis of 2,3-dihydrofuran derivatives 152 (Scheme 50). ${ }^{392} \mathrm{~A}$ variety of alkenes and cycloalkenes bearing electron-withdrawing or electron-donating substituents can be used in this cyclization.

Wirth and co-workers reported the lactonization of 4-phenyl-4-pentenoic acid 153 upon treatment with DIB (Scheme 51). ${ }^{393}$ The mechanism of this reaction includes electrophilic lactonization induced by the addition of the iodine(III) electrophile to the double bond of substrate $\mathbf{1 5 3}$ followed by 1,2-phenyl migration leading to the final rearranged lactone $\mathbf{1 5 4}$. The same group reported a one-pot procedure for the conversion of alkenes into 1,1dicyanocyclopropane derivatives by treatment with DIB and 1,1-dicyanopropane. ${ }^{394}$

Kita and co-workers developed a facile and efficient synthesis of lactols 156 via an oxidative rearrangement reaction of 2,3-epoxy alcohols 155 with BTI (Scheme 52). ${ }^{395-397}$ This BTIinduced oxidative transformation has been utilized in the synthesis of several lactones and in the asymmetric synthesis of the marine $\gamma$-lactone metabolite (+)-tanikolide. ${ }^{395,396}$

A DIB-induced domino reaction of the vicinal unsaturated diol 157 afforded cyclic ene-acetal 158 (Scheme 53), which was further utilized in the synthesis of a norsesquiterpene spirolactone/ testosterone hybrid. ${ }^{398}$

Iglesias-Arteaga and co-workers reported several DIB-promoted oxidative transformations of steroidal substrates. ${ }^{399-401}$ In particular, the treatment of (25R)-3 $\alpha$-acetoxy-5 $\beta$-spirostan-23one 159 with DIB in basic methanol leads to F-ring contraction via Favorskii rearrangement to afford product $\mathbf{1 6 0}$ (Scheme 54). ${ }^{399}$

The treatment of steroidal substrate $\mathbf{1 6 1}$ with DIB and boron trifluoride etherate in acetic acid led to the introduction of an axial acetoxy group at position C-23 of the side chain, ${ }^{400}$ while a similar reaction of the same substrate 161 with $\mathrm{DIB}$ and $\mathrm{BF}_{3} \cdot \mathrm{OEt}_{2}$ in formic acid unexpectedly produced the equatorial formate $\mathbf{1 6 2}$ mixed with products of rearrangement 163 and 164 (Scheme 55). 401

The DIB-promoted oxidative iodolactonization of pentenoic acids $\mathbf{1 6 5}$ in the presence of tetrabutylammonium iodide proceeds smoothly at room temperature to afford lactones 166 in high yields. ${ }^{402}$ Based on this reaction, a convenient approach has been developed for the iodolactonization using iodobenzene as a catalyst (Scheme 56). In this procedure, DIB is generated in situ using a catalytic amount of iodobenzene with sodium perborate monohydrate as the stoichiometric oxidant. A variety of unsaturated acids including $\delta$-pentenoic acids 167, $\delta$-pentynoic acids and $\delta$-hexynoic acid gave high yields of the respective lactones (e.g. 168) using this organocatalytic methodology (Scheme 56). ${ }^{402}$

Kita and co-workers reported a mild and efficient fragmentation reaction of $\beta$-amino alcohols 169 and $\alpha$-amino acids 170 upon treatment with [bis(trifluoroacetoxy)iodo] pentafluorobenzene leading to N,O-acetals 171 (Scheme 57). This method has been utilized in an improved synthesis of the key intermediate of discorhabdins. ${ }^{403,404}$

Kozlowski and co-workers reported an unusual DIB-promoted oxidative rearrangement of cis- and trans-1,5-diazadecalins. In a specific example, upon treatment with DIB in aqueous $\mathrm{NaOH}, 1,5$-diaza-trans-decalin $\mathbf{1 7 2}$ undergoes oxidation along with fragmentation to yield the ring-expanded bislactam 173 (Scheme 58). ${ }^{405}$

A stereoselective synthesis of 5-7 membered cyclic ethers can be achieved by deiodonative ring-enlargement of cyclic ethers having an iodoalkyl substituent. For example, the reaction 
of tetrahydrofuran derivative $\mathbf{1 7 4}$ with (diacetoxyiodo)toluene proceeds under mild conditions to afford ring-expanded product 175 (Scheme 59). The use of hexafluoroisopropanol (HFIP) as solvent in this reaction is critically important. ${ }^{406}$

[Bis(acyloxy)iodo]arenes can serve as excellent oxidants in Hofmann-type degradation of aliphatic or aromatic carboxamides to the respective amines. DIB is a superior reagent for the Hofmann rearrangement of protected asparagines. ${ }^{407}$ This procedure was used for the preparation of optically pure $N_{\alpha}-n$-Boc-L- $\alpha, \beta$-diaminopropionic acid $\mathbf{1 7 7}$ from asparagine $\mathbf{1 7 6}$ in hundred kilogram quantities (Scheme 60 ). ${ }^{408}$ Other examples include the oxidative rearrangement of anthranilamides or salicylamides $\mathbf{1 7 8}$ to the respective heterocycles 179, 409 and the preparation of alkyl carbamates of 1-protected indole-3-methylamines $\mathbf{1 8 1}$ from the corresponding acetamides 180 (Scheme 60). ${ }^{410}$

BTI has also been used as a reagent for the Hofmann rearrangement, as illustrated by the conversion of amide $\mathbf{1 8 2}$ to the respective amine $\mathbf{1 8 3}$ (Scheme 61). ${ }^{411}$ A similar BTI-induced Hofmann rearrangement has been used for the preparation of both enantiomers of trans-2aminocyclohexanecarboxylic acid from trans-cyclohexane-1,2-dicarboxylic acid. ${ }^{412}$

3.4.6. Oxidative Dearomatization of Phenolic Substrates-[Bis(acyloxy)iodo]arenes are commonly used as the reagents for various synthetically useful oxidative transformations of phenolic compounds. ${ }^{32,34,50,51,53,60}$ DIB is the reagent of choice for the oxidation of various substituted $o$ - and $p$-hydroquinones to the corresponding benzoquinones. The oxidation generally proceeds in methanol solution at room temperature, and the yield of benzoquinones is almost quantitative. ${ }^{413}$ Gladysz and Rocaboy have reported the application of fluorous (diacetoxyiodo)arenes in oxidations of hydroquinones to quinones; in this procedure the fluorous reagents can be conveniently recovered by simple liquid/liquid biphase workups. ${ }^{273}$ Particularly useful is the oxidative dearomatization of 4- or 2-substituted phenols (e.g. 184 and 188) with DIB or BTI in the presence of an appropriate external or internal nucleophile $(\mathrm{Nu})$ leading to the respective cyclohexadienones 187 or 189 according to Scheme 62. The mechanism of this reaction most likely involves the initial formation of the phenoxyiodine(III) species $\mathbf{1 8 5}$ followed by elimination of $\mathrm{PhI}$ and the generation of cationic phenoxenium intermediates 186, which finally combine with the nucleophile. ${ }^{5,414}$

Various nucleophiles, such as water, ${ }^{415}$ alcohols, ${ }^{76,413,416-418}$ fluoride ion, ${ }^{419}$ carboxylic acids, ${ }^{418,420,421}$ amides, ${ }^{422}$ oximes, ${ }^{423}$ and electron-rich aromatic rings, ${ }^{424,425}$ have been used successfully in this reaction (Scheme 62) in either an inter- or intra-molecular mode. Recent examples of this reaction in the inter-molecular mode include the oxidative ipso-fluorination of $p$-substituted phenols 190 (or a similar ipso-fluorination of $p$-substituted anilines ${ }^{426}$ ) using pyridinium polyhydrogen fluoride, $\mathrm{Py} \bullet(\mathrm{HF})_{\mathrm{x}}$, in combination with DIB or BTI, ${ }^{427}$ and the methoxylation of various phenolic substrates, such as 191, using DIB in methanol (Scheme 63). ${ }^{428-430}$ This reaction can be further improved by using phenol trimethylsilyl ethers instead of phenols as the substrates. It was shown that the oxidation of trimethylsilyl ethers 192 affords $p$-quinols 193 in greatly improved yields due to the minimization of oligomer side products formation compared to the oxidation of free phenol. ${ }^{431}$

Very recently, Quideau and co-workers have reported the preparation of versatile chiral substrates for asymmetric synthesis through the DIB induced spiroketalization of phenols with a chiral substituted ethanol unit $O$-tethered to the ortho position. ${ }^{76}$ This reaction has been successfully utilized in the asymmetric total synthesis of the natural product (+)-biscarvacrol.

Quideau and co-workers have developed a BTI-mediated regioselective protocol for the oxidative dearomatization of 2-alkoxyarenols in the presence of external carbon-based nucleophiles. ${ }^{432-435}$ This is a synthetically valuable process, as illustrated by the BTI-mediated 
oxidative nucleophilic substitution of the 2-alkoxynaphthol 194 with the silyl enol ether 195 leading to the highly functionalized naphthoid cyclohexa-2,4-dienone 196 (Scheme 64), which is an important intermediate product in the synthesis of aquayamycin-type angucyclinones. 434,435

The DIB or BTI-induced phenolic oxidation in the intra-molecular mode provides an efficient approach to synthetically valuable polycyclic products. Representative examples of oxidative phenolic cyclizations promoted by [bis(acyloxy)iodo]arenes are shown in Scheme 65. In particular, the oxidative cyclization of phenolic oxazolines 197 affords synthetically useful spirolactams $\mathbf{1 9 8}, 51,436$ the oxidation of enamide $\mathbf{1 9 9}$ leads to the spiroenamide $\mathbf{2 0 0}$, which is a key intermediate product in the total synthesis of annosqualine, ${ }^{437}$ and the spirocyclic product 202 has been prepared by a BTI-induced oxidation of catechol 201 in a key step of the total synthesis of the marine sesquiterpene quinone (+)-puupehenone. ${ }^{438}$

Additional examples of the DIB or BTI-induced oxidative phenolic cyclizations include the following studies: the asymmetric total syntheses of the pentacyclic Stemona alkaloids tuberostemonine and didehydrotuberostemonine, ${ }^{439}$ the fully stereocontrolled total syntheses of (-)-cylindricine $\mathrm{C}$ and (-)-2-epicylindricine $\mathrm{C},{ }^{440,441}$ the asymmetric total syntheses of platensimycin, ${ }^{442}$ the total synthesis of a potent antitumor alkaloid, discorhabdin $\mathrm{A},{ }^{443}$ the total synthesis of the amaryllidaceae alkaloid (+)-plicamine using solid-supported reagents, ${ }^{444}$ the construction of oxygenated indole, quinoline, and phenanthridine alkaloid motifs, ${ }^{445}$ DIBmediated regioselective aza benzannulation of nitrogen-tethered 2-methoxyphenols, ${ }^{446}$ the investigation of oxidative dearomatization of resorcinol derivatives leading to valuable cyclohexa-2,5-dienones, ${ }^{447}$ the development of enantioselective organocatalytic oxidative dearomatization methodology, ${ }^{448}$ the development of a flow process for the multi-step synthesis of the alkaloid natural product oxomaritidine, ${ }^{449}$ the synthesis of carpanone using solid-supported reagents and scavengers, ${ }^{450}$ and the studies on ring expansions of a spirocyclohexadienone system. ${ }^{451}$

Kita and co-workers have reported a catalytic variant of the oxidative spirocyclization reaction based on the in situ regeneration of a [bis(trifluoroacetoxy)iodo]arene from iodoarene using $m$-chloroperbenzoic acid ( $m$ CPBA) as a terminal oxidant ${ }^{452}$ In a typical example, the oxidation of the phenolic substrate $\mathbf{2 0 3}$ with $m \mathrm{CPBA}$ in dichloromethane in the presence of a catalytic amount of $p$-[bis(trifluoroacetoxy)iodo]toluene ( 0.01 equiv.) and trifluoroacetic acid at room temperature affords the respective spirolactone 204 in good yield (Scheme 66). A variety of other [bis(trifluoroacetoxy)iodo]arenes can be used as catalysts in this reaction [e.g. BTI, 4$\mathrm{MeOC}_{6} \mathrm{H}_{4} \mathrm{I}\left(\mathrm{OCOCF}_{3}\right)_{2}$ and $\left.2,4-\mathrm{F}_{2} \mathrm{C}_{6} \mathrm{H}_{3} \mathrm{I}\left(\mathrm{OCOCF}_{3}\right)_{2}\right]$ and different acidic additives (acetic acid, $\mathrm{BF}_{3} \bullet \mathrm{OEt}_{2}$, TMSOTf, molecular sieves), but the TolI $\left(\mathrm{OCOCF}_{3}\right)_{2} / \mathrm{CF}_{3} \mathrm{CO}_{2} \mathrm{H}$ system generally provides the best catalytic efficiency. Under these optimized conditions, a variety of phenolic substrates 205 was oxidized to spirolactones $\mathbf{2 0 6}$ in the presence of catalytic amounts of $p$-iodotoluene (Scheme 66). ${ }^{452}$ Likewise, the amide derivatives of phenolic substrates 205 can be catalytically oxidized to the respective $N$-fused spirolactams using catalytic amounts of $p$-iodotoluene and $m \mathrm{CPBA}$ as a terminal oxidant. ${ }^{453} \mathrm{~A}$ similar catalytic procedure has been reported for the oxidation of 4-alkoxyphenols to the corresponding 1,4-quinones using a catalytic amount of 4-iodophenoxyacetate in the presence of oxone as a co-oxidant in an aqueous acetonitrile solution. ${ }^{454}$

Very recently, Kita and co-workers reported the first enantioselective spirocyclization reaction of the ortho-substituted phenolic substrates using chiral aryliodine(III) diacetate having a rigid spirobiindane backbone. ${ }^{455}$

The oxidative dearomatization of substituted phenols 188 bearing electron-releasing substituents R, such as methoxy group, at their ortho-position(s) leads to 6,6-disubstituted 
cyclohexa-2,4-dienones 189 (see Scheme 62), which can be conveniently utilized in situ as dienes in Diels-Alder reactions. ${ }^{418,421,456}$ When the oxidation of phenols is performed in the absence of an external dienophile, a dimerization via [4+2] cycloaddition often occurs spontaneously at ambient temperature to afford the corresponding dimers with an extraordinary level of regio-, site-, and stereoselectivity. A detailed experimental and theoretical investigation of such hypervalent iodine induced Diels-Alder cyclodimerizations has recently been published by Quideau and co-workers. ${ }^{456}$ A representative example of an oxidative DielsAlder cyclodimerization of a phenolic substrate $\mathbf{2 0 7}$ to the dimer $\mathbf{2 0 8}$ is shown in Scheme 67.

When the oxidation is performed in the presence of an external dienophile, the respective products of [4+2] cycloaddition are formed. ${ }^{457-461}$ Typical examples are illustrated by a onepot synthesis of several silyl bicyclic alkenes $\mathbf{2 1 1}$ by intermolecular Diels-Alder reactions of 4-trimethylsilyl substituted masked $\boldsymbol{o}$-benzoquinones $\mathbf{2 1 0}$ derived from the corresponding 2methoxyphenols 209,457 and by the hypervalent iodine-mediated oxidative dearomatization/ Diels-Alder cascade reaction of phenols 212 with allyl alcohol affording polycyclic acetals 213 (Scheme 68). ${ }^{458}$ The BTI-promoted tandem phenolic oxidation/Diels-Alder reaction has been utilized in the stereoselective synthesis of the bacchopetiolone carbocyclic core. ${ }^{459}$

A mechanistic investigation of the oxidation of 2,6-dimethylphenol using different oxidizing systems has shown that DIB is the most efficient reagents for the oxidative coupling leading to $3,5,3^{\prime}, 5^{\prime}$-tetramethyl-biphenyl-4,4'-diol. A reaction mechanism was proposed which involved an initial formation of a [bis(phenoxy)iodo]benzene intermediate followed by its radical fragmentation and then radical coupling and comproportionation/redox reaction steps. 462

3.4.7. Oxidative Coupling of Electron-Rich Aromatic Substrates-The interaction of phenol ethers $\mathbf{2 1 4}$ or other electron-rich aromatic substrates with BTI leads to the generation of cation radical intermediates $\mathbf{2 1 5}$, which combine with external or internal nucleophiles affording the products of dearomatization 216 or coupling 217 according to Scheme 69. Kita and co-workers have recently published a detailed mechanistic study of this process (Scheme 69) for a specific reaction of oxidative cyclization of electron-rich aromatics with the intramolecular hydroxyl group. ${ }^{463}$ In this study, the formation of the cation radical intermediates $215\left(\mathrm{R}-\mathrm{Nu}=-\mathrm{CH}_{2} \mathrm{CH}_{2} \mathrm{CH}_{2} \mathrm{OH}\right)$ was experimentally confirmed by ESR spectroscopy, and the factors determining the ratio of products 216 and 217 and their consequent transformations were clarified.

The direct nucleophilic substitution of electron-rich phenol ethers using BTI and Lewis acid and involving aromatic cation radical intermediates was originally developed by Kita and coworkers in $1994 .{ }^{464}$ Since then this procedure with some variations has been extensively applied by Kita and other researchers for various oxidative transformations, such as the synthesis of biaryls, ${ }^{465-472}$ spirodienones, ${ }^{467,473-475}$ quinone imines, ${ }^{476}$ sulfur-containing heterocycles, ${ }^{477}$ and chromans. ${ }^{478}$ Specific recent examples of the oxidative coupling of phenolic ethers include the oxidative biaryl coupling of various N-substituted 1benzyltetrahydroisoquinolines 218 to the corresponding aporphines 219,468 the oxidative cyclization of 3,4-dimethoxyphenyl 3,4-dimethoxyphenylacetate 220 leading the sevenmembered lactone 221,469 and the conversion of phenol ether derivatives 222 to the products of intramolecular coupling $\mathbf{2 2 3}$ using a combination of BTI and heteropoly acid (Scheme 70). 466 A similar oxidative coupling reaction of benzyltetrahydroisoquinolines (laudanosine derivatives) using BTI and heteropoly acid has been used in an efficient synthesis of morphinandienone alkaloids. ${ }^{479}$ A catalytic version of the intermolecular oxidative coupling of phenolic ethers using BTI ( 0.125 equivalents) as a catalyst and $m \mathrm{CPBA}$ as the stoichiometric oxidant has also been reported. ${ }^{452}$ Very recently, Kita and co-workers have reported a new 
$\mathrm{H}_{2} \mathrm{O}_{2}$ /acid anhydride system for the iodoarene-catalyzed intramolecular C-C cyclization of phenolic derivatives. ${ }^{480}$

The non-phenolic electron-rich aromatic substrates can also be oxidatively coupled using [bis (acyloxy)iodo]arenes. Kita and co-workers reported facile and efficient oxidative coupling reaction of alkylarenes $\mathbf{2 2 4}$ leading to alkylbiaryls $\mathbf{2 2 5}$ using a combination of BTI and $\mathrm{BF}_{3} \cdot \mathrm{OEt}_{2}$ (Scheme 71$){ }^{481}$ Similarly, multiply iodinated biaryls can be prepared in good yields by the BTI-induced direct oxidative coupling reaction of the iodinated arenes. ${ }^{482}$

Oxidation of $\mathrm{N}$-aromatic methanesulfonamides 226 with DIB in the presence of thiophene in trifluoroethanol or hexafluoroisopropanol affords the respective coupling products 227 in good yield. ${ }^{483}$ Likewise, the head-to-tail dimers $\mathbf{2 2 9}$ can be selectively prepared by the hypervalent iodine oxidation of 3-substituted thiophenes 228,484,485 and bipyrroles 231 can be regioselectively synthesized by oxidative dimerization of pyrroles $\mathbf{2 3 0}$ with BTI in the presence of bromotrimethylsilane (Scheme 72). ${ }^{486}$

\subsubsection{Radical Cyclizations, Rearrangements and Fragmentations-Useful} synthetic methodologies are based on the cyclization, rearrangement or fragmentation of the alkoxyl radicals generated in the reaction of alcohols with [bis(acyloxy)iodo]arenes in the presence of iodine under photochemical conditions or in the absence of irradiation. ${ }^{5,6}$ Suàrez and co-workers have applied this methodology in various useful transformations of carbohydrate derivatives, such as the synthesis of polyhydroxy piperidines and pyrrolidines related to carbohydrates, ${ }^{129}$ the synthesis of alduronic acid lactones, ${ }^{487}$ the syntheses of chiral dispiroacetals from carbohydrates, ${ }^{488}$ and the synthesis of $\alpha$-iodoalkyl esters from carbohydrates. ${ }^{489}$ Recent examples include the synthesis of 1,1-difluoro-1-iodo alditols $\mathbf{2 3 3}$, 490 2-azido-1,2-dideoxy-1-iodo-alditols $\mathbf{2 3 5}, 491,492$ and chiral vinyl sulfones $\mathbf{2 3 7} \mathbf{3}^{493}$ by fragmentation of carbohydrate anomeric alkoxyl radicals generated from the respective carbohydrates 232, 234 and 236 (Scheme 73).

The intramolecular hydrogen abstraction reactions promoted by alkoxy radicals in carbohydrates are particularly useful for the stereoselective synthesis of various polycyclic oxygen-containing ring systems. ${ }^{128,494-497}$ This reaction can be illustrated by the intramolecular 1,8-hydrogen abstraction between glucopyranose units in disaccharide $\mathbf{2 3 8}$ promoted by alkoxyl radicals and leading to the 1,3,5-trioxocane derivative $\mathbf{2 3 9}$ (Scheme 74). 494

Boto and Hernandez have reported a short and efficient synthesis of chiral furyl carbinols from carbohydrates, such as $\mathbf{2 4 0}$, based on the alkoxyl radicals fragmentation reaction leading to the intermediate product 241 (Scheme 75). ${ }^{498}$ The same authors have developed an efficient procedure for the selective removal from carbohydrate substrates of methoxy protecting groups next to hydroxy groups by treatment with the DIB-I $\mathrm{I}_{2}$ system. ${ }^{499}$

The treatment of 1-alkynylcycloalkanols $\mathbf{2 4 2}$ with poly[styrene(iodosodiacetate)] and iodine affords (Z)-2-(1-iodo-1-organyl)methylenecycloalkanones 243 resulting, probably, from the alkoxyl radical promoted ring expansion reaction (Scheme 76). ${ }^{500}$ The mechanism of the $\beta$ scission reactions of the 1-alkylcycloalkoxyl radicals generated from alkylcycloalkanols by treatment with the DIB- $\mathrm{I}_{2}$ under photochemical conditions has been investigated by Bietti and co-workers. ${ }^{501}$

A mild and highly efficient one-pot synthesis of aryl glycines $\mathbf{2 4 5}$ from easily available serine derivatives $\mathbf{2 4 4}$ has been reported (Scheme 77). ${ }^{502}$ The method is based on the $\beta$-fragmentation of a primary alkoxyl radical, generated on treatment of the serine derivative with DIB and 
iodine, immediately followed by the addition of the nucleophile. This methodology is also applicable to the synthesis of other uncommon amino acids. ${ }^{502}$

The one-pot radical fragmentation-phosphorylation reaction of $\alpha$-amino acids or $\beta$-amino alcohols (e.g. 246) affords $\alpha$-amino phosphonates 247 in good yields (Scheme 78). This reaction was applied to the synthesis of potentially bioactive phosphonates. ${ }^{503}$

The radical decarboxylation of carboxylic acids on treatment with DIB- $\mathrm{I}_{2}$ allows to introduce iodine or other functional group into nitrogen heterocycles under mild conditions. ${ }^{504,505}$ For example, the decarboxylation of $\beta$ - and $\gamma$-amino acids $\mathbf{2 4 8}$ under these conditions affords iodinated heterocycles $\mathbf{2 4 9}$ (Scheme 79). This reaction was applied to the synthesis of bioactive products, such as opioid analogs, imino sugars and new antifungal agents. ${ }^{504}$

Kita and co-workers developed a simple and reliable method for the direct construction of biologically important aryl lactones $\mathbf{2 5 1}$ from carboxylic acids $\mathbf{2 5 0}$ using a combination of DIB with $\mathrm{KBr}$ (Scheme 80 ). The mechanism of this reaction includes the initial generation of the carbonyloxy radical followed by the intramolecular benzylic hydrogen abstraction and cyclization. ${ }^{506}$

Conjugate addition of radicals generated by decarboxylative fragmentation of(diacyloxyiodo) benzene 103 to dehydroamino acid derivatives (e.g. 252) has been used by Sutherland and Vederas in the synthesis of diaminopimelic acid analogues 253 (Scheme 81). ${ }^{278}$

Barluenga and co-workers reported a direct iodination of alkanes 254 by the reaction with DIB$\mathrm{I}_{2}$ in the presence of $t$-butanol under photochemical or thermal conditions (Scheme 82). ${ }^{507}$ This reaction can be used for the preparation of alkyliodides 255 in excellent yields by direct $\mathrm{C}-\mathrm{H}$ bond activations in cyclic or non-cyclic alkanes and at the benzylic position. The presence of an alcohol (e.g., $t$-butanol) is essential for an efficient alkane activation.

The alkoxy radical fragmentation with DIB in the presence of iodine was also used in a facile synthesis of $(n+3)$ and $(n+4)$ ring-enlarged lactones as well as of spiroketolactones from nmembered cycloalkanones. ${ }^{508}$

Useful synthetic methodologies are based on the cyclization or rearrangement of the nitrogencentered radicals generated in the reaction of the appropriate amides with DIB in the presence of iodine. ${ }^{130,509-511}$ Specific examples are illustrated by the synthesis of bicyclic spirolactams 257 from amides 256, 509 and the preparation of the oxa-azabicyclic systems (e.g. 259) by the intramolecular hydrogen atom transfer reaction promoted by carbamoyl and phosphoramidyl radicals generated from the appropriately substituted carbohydrates $\mathbf{2 5 8}$ (Scheme 83). ${ }^{510}$

3.4.9. Oxidations of Nitrogen, Phosphorus, and Sulfur Compounds-DIB and BTI have found wide application for the oxidation of organic derivatives of such elements as nitrogen, sulfur, selenium, tellurium, and others. ${ }^{5,6}$ The use of [bis(acyloxy)iodo]arenes for the oxidation of organonitrogen compounds leading to the generation of the $\mathrm{N}$-centered cationic or radical intermediates and their subsequent cyclizations and rearrangements (e.g. Hofmann rearrangement) is discussed in previous sections of this review (see Sections 3.4.5 and 3.4.8). Additional recent examples include the DIB induced oxidation of aromatic amines to imines applied for deprotection of protected amino diols, ${ }^{512}$ the $\mathrm{N}$-acylation of 1,3-disubstituted thioureas using DIB, ${ }^{513}$ the DIB oxidation of 1,2-dicarbethoxyhydrazine to diethyl azodicarboxylate as a key step of an organocatalytic Mitsunobu reaction,, 14 the BTI oxidations of phenylhydrazones leading to regeneration of the carbonyl function, ${ }^{515}$ the low temperature generation of diazocompounds by the reaction of BTI with hydrazones, ${ }^{516}$ the preparation of $N$-aroyl- $N$ '-arylsulfonylhydrazines by oxidation of aromatic aldehyde $N$ - 
arylsulfonylhydrazones with BTI, 517 and conversion of oximes into nitroso compounds using p-bromo(diacetoxyiodo)benzene. ${ }^{518}$

[Bis(acyloxy)iodo]arenes have been used for the oxidation of various organosulfur compounds. Organic sulfides are selectively oxidized to the respective sulfoxides by DIB or the polymersupported DIB in water in the presence of $\mathrm{KBr}^{5}{ }^{519}$ The recyclable reagent, 3-[bis (trifluoroacetoxy)iodo]benzoic acid 109, can oxidize organic sulfides to the respective sulfoxides at room temperature in aqueous acetonitrile. ${ }^{103}$ Thioacetals and thioketals are efficiently cleaved to carbonyl compounds with BTI or DIB under mild conditions. This reaction is especially useful for the selective deprotection of either thioacetals or thioketals and is compatible with a variety of other functional groups. ${ }^{520-524}$

Makowiec and Rachon investigated the reactivity of DIB toward trivalent phosphorus nucleophiles. It was found that both $\mathrm{H}$-phosphonates and secondary phosphine oxides react with DIB in alcohols in the presence of sodium alkoxides yielding trialkyl phosphates and alkyl phosphinates, respectively. A mechanism of these reactions involving an initial addition of a phosphorus(III) nucleophile to the iodine(III) center has been proposed. ${ }^{525}$

3.4.10. Transition Metal Catalyzed Reactions-The oxidations with [bis(acyloxy)iodo] arenes can be effectively catalyzed by transition metal salts and complexes. DIB is occasionally used instead of iodosylbenzene as the terminal oxidant in biomimetic oxygenations catalyzed by metalloporphyrins and other transition metal complexes. ${ }^{526-528}$ Primary and secondary alcohols can be selectively oxidized to the corresponding carbonyl compounds by DIB in the presence of transition metal catalysts, such as $\mathrm{RuCl}_{3}, 139,529 \mathrm{Ru}$ (Pybox)(Pydic) complex, 530 polymer-micelle incarcerated ruthenium catalysts, ${ }^{531}$ chiral-Mn(salen)-complexes, ${ }^{532,533} \mathrm{Mn}$ (TPP)CN/Im catalytic system, ${ }^{534}$ and (salen)Cr(III) complexes. ${ }^{535}$ Kirschning and co-workers have recently reported the use of the recyclable reagent, phenylsulfonate-tagged DIB, in the $\mathrm{RuCl}_{3}$-catalyzed oxidation of alcohols. ${ }^{536}$ The epoxidation of alkenes, such as stilbenes, indene and 1-methylcyclohexene, using DIB in the presence of chiral binaphthyl ruthenium(III) catalysts $(5 \mathrm{~mol} \%)$ has also been reported. The chemoselectivity and enantioselectivity of this reaction was found to be low ( $4 \%$ ee)..$^{537}$

The mechanisms and applications of palladium-catalyzed reactions of DIB and other hypervalent iodine reagents in synthetically useful organic transformations were recently reviewed by Deprez and Sanford. ${ }^{18}$ Particularly useful are the Pd-catalyzed oxidation reactions, including the oxidative functionalization of $\mathrm{C}-\mathrm{H}$ bonds and the 1,2aminooxygenation of olefinic substrates ${ }^{538-552}$ Representative examples of these catalytic oxidations are illustrated by the selective acetoxylation of $\mathrm{C}-\mathrm{H}$ bonds adjacent to coordinating functional groups (e.g., pyridine in substrate 260), ${ }^{538}$ and by the $\mathrm{Pd}(\mathrm{OAc})_{2}$-catalyzed intramolecular aminoacetoxylation in the reaction of $\gamma$-aminoolefins (e.g., cinnamyl alcohol derived tosyl carbamate 261) with DIB (Scheme 84). ${ }^{539}$ The key mechanistic step in these catalytic transformations includes the DIB promoted oxidation of Pd(II) to the Pd(IV) species, as proved by the isolation and $\mathrm{X}$-ray structural identification of stable $\mathrm{Pd}(\mathrm{IV})$ complexes prepared by the reaction of $\mathrm{PhI}\left(\mathrm{O}_{2} \mathrm{CPh}\right)_{2}$ with $\mathrm{Pd}$ (II) complexes containing chelating 2phenylpyridine ligands. ${ }^{553}$

Yan and co-workers have developed an efficient procedure for synthesis of symmetrical conjugated diynes 263 from terminal alkynes 262 using DIB as oxidant under palladiumcatalyzed conditions (Scheme 85). ${ }^{554,555}$

\subsection{Organosulfonates}

A detailed discussion of the literature on the preparation, structural studies and synthetic applications of aryliodine(III) compounds derived from strong inorganic acids can be found in 
our previous reviews. ${ }^{5,6}$ The aryliodine(III) compounds $\mathrm{ArI}(\mathrm{OX})_{2}$ that are derived from strong acids $\mathrm{HOX}$, such as $\mathrm{H}_{2} \mathrm{SO}_{4}, \mathrm{HNO}_{3}, \mathrm{HClO}_{4}, \mathrm{CF}_{3} \mathrm{SO}_{3} \mathrm{H}, \mathrm{HSbF}_{6}$ and $\mathrm{HPF}_{6}$, usually lack stability and can only be generated at low temperature, under absolutely dry conditions. Traces of moisture immediately convert these compounds into $\mu$-oxo-bridged derivatives or more complex polymeric structures (see structures 8 and 9 in Section 3.1.2). For example, the unstable and extremely hygroscopic phenyliodine(III) sulfates $\mathrm{PhIO} \bullet \mathrm{SO}_{3}$ and $(\mathrm{PhIO})_{2} \bullet \mathrm{SO}_{3}$ can be generated from $\mathrm{PhIO}$ and $\mathrm{SO}_{3}$ or $\mathrm{Me}_{3} \mathrm{SiOSO}_{2} \mathrm{Cl}$ under absolutely dry conditions, ${ }^{556-}$ 558 while the partially hydrolyzed, stable oligomeric sulfate $(\mathrm{PhIO})_{3} \cdot \mathrm{SO}_{3}$ (structure 8 ) is conveniently prepared by the treatment of $\mathrm{PhI}(\mathrm{OAc})_{2}$ with aqueous $\mathrm{NaHSO}_{4} \cdot{ }^{88}$

[Hydroxy(organosulfonyloxy)iodo]arenes, $\mathrm{ArI}(\mathrm{OH}) \mathrm{OSO}_{2} \mathrm{R}$, are the most common, well investigated, and practically useful aryliodine(III) derivatives of strong acids. The most important of them, [hydroxy(tosyloxy)iodo]benzene (HTIB or Koser's reagent), is commercially available and is commonly used as an oxidizing reagent in organic synthesis.

${ }^{41}$ In this section, the preparation, structural studies, and recent examples of synthetic applications of [hydroxy(organosulfonyloxy)iodo]arenes are overviewed.

3.5.1. Preparation-Various [hydroxy(tosyloxy)iodo]arenes are readily prepared by a ligand exchange reaction of (diacetoxyiodo)arenes with $p$-toluenesulfonic acid monohydrate in acetonitrile (Scheme 86). ${ }^{75,103,257,260,261,559,560}$ This method has recently been applied to the synthesis of [hydroxy(tosyloxy)iodo] heteroaromatic derivatives (e.g., 264 and 265), 560 the derivatives with various substituted aromatic groups (e.g. 266 and 267), ${ }^{103,257,560}$ and the recyclable hypervalent iodine reagents 268 and $\mathbf{2 6 9} .{ }^{260,261}$ A convenient modified procedure for the preparation of various [hydroxy(sulfonyloxy)iodo]arenes consists of the one-pot reaction of iodoarenes and $m \mathrm{CPBA}$ in the presence of sulfonic acids in a small amount of chloroform at room temperature. ${ }^{561}$ This modified procedure was recently used for the preparation of new biphenyl- and terphenyl-based recyclable organic trivalent iodine reagents 270 and 271. ${ }^{264}$

A similar procedure using 4-nitrobenzenesulfonic acid, methanesulfonic acid, or 10camphorsulfonic acid leads to the corresponding organosulfonyloxy analogs. ${ }^{559,562} \mathrm{~A}$ solventfree, solid-state version of this reaction is carried out by simple grinding of $\operatorname{ArI}(\mathrm{OAc})_{2}$ with the appropriate sulfonic acid in an agate mortar followed by washing the solid residue with diethyl ether. ${ }^{563}$ This solid-state procedure has been used for the preparation of HTIB and several other [hydroxy(organosulfonyloxy)iodo]arenes in 77-98\% yields. A polymersupported [hydroxy(tosyloxy)iodo]benzene can be prepared similarly by treatment of poly [(diacetoxy)iodo]styrene with $p$-toluenesulfonic acid monohydrate in chloroform at room temperature. ${ }^{564,565}$

The highly electrophilic phenyliodine(III) trifluoromethanesulfonate $(\mathrm{PhIO})_{2} \bullet \mathrm{Tf}_{2} \mathrm{O}$, which is also known as Zefirov's reagent, may be prepared either by the exchange reaction of (diacetoxy)iodobenzene with trifluoromethanesulfonic acid, ${ }^{566}$ or by the combination of two equivalents of iodosobenzene with one equivalent of triflic anhydride. ${ }^{567}$ This triflate has an oxo-bridged structure and is isolated as a relatively stable yellow microcrystalline solid that can be handled for brief periods in air and stored under a nitrogen atmosphere. It can be conveniently generated in situ from $\mathrm{PhIO}$ and triflic anhydride or trimethylsilyl triflate and immediately used in the subsequent reactions; ${ }^{568}$ the extended storage of this reagent in the presence of trifluoromethanesulfonic acid results in self-condensation with the formation of oligomeric products. ${ }^{569}$

3.5.2. Structural Studies-Single-crystal X-ray structural data for HTIB show the Tshaped geometry around the iodine center with almost collinear O-ligands and two different I-O bonds of $2.47 \AA$ (I-OTs) and $1.94 \AA$ (I-OH).${ }^{570}$ The presence of a substituent in the phenyl 
ring does not have any noticeable effect on the molecular geometry of [hydroxy(tosyloxy)iodo] arenes. The recently reported X-ray structure of 3-[hydroxy(tosyloxy)iodo]benzoic acid $\mathbf{2 6 7}$ is very similar to the structure of HTIB. The I-OTs bond distance in tosylate 267, $(2.437 \AA)$, is significantly longer than the I-OH bond distance of $1.954 \AA$, which is indicative of some ionic character of this compound. In addition to the three intramolecular bonds, a weaker intermolecular coordination of iodine atom to one of the sulfonyl oxygens of the neighboring molecule is found with a distance of $2.931 \AA$. No intermolecular interaction involving a meta carboxylic group is present in molecule 267. ${ }^{103}$

The solution studies of HTIB in water by spectroscopic measurements and potentiometric titrations indicate complete ionization to a hydroxy(phenyl)iodonium cation $\left(\mathrm{PhI}^{+} \mathrm{OH}\right.$ in hydrated form) and tosylate anion. ${ }^{111}$

3.5.3. Reactions-The functionalization of carbonyl compounds at an $\alpha$-carbon represents the most typical reaction of [hydroxy(organosulfonyloxy)iodo] arenes (Scheme 87). ${ }^{41}$ Recent examples of synthetic application of this procedure include the following: the preparation of $\alpha$-mesyloxyketones for the photochemical synthesis of highly functionalized cyclopropyl ketones ${ }^{571}$ the one-step conversion of ketones into $\alpha$-azidoketones using HTIB and sodium azide, ${ }^{572,573}$ the one-pot conversion of ketones into $\beta$-keto sulfones using HTIB and sodium arene sulfinate under solvent-free conditions, ${ }^{574}$ the solvent-free synthesis of $\alpha$-tosyloxy $\beta$ keto sulfones using HTIB, ${ }^{575}$ direct $\alpha$-hydroxylation of ketones using HTIB or polymersupported HTIB in dimethyl sulfoxide-water, ${ }^{576,577}$ the use of HTIB in the synthesis of 1,4diaryl-2-(arylamino)-but-2-ene-1,4-diones, ${ }^{578}$ the high yield preparation of dicarboxylic acid dimethyl esters from cycloalkanones using [hydroxy(2,4-dinitrobenzenesulfonyloxy)iodo] benzene, ${ }^{579}$ the ionic liquid-accelerated one-pot synthesis of 2-arylimidazo[1,2-a]pyrimidines, 580 the HTIB mediated stereoselective synthesis of bicyclic ketones, ${ }^{581}$ the HTIB promoted synthesis of 6-arylimidazo[2,1-b]thiazoles, ${ }^{582}$ the synthesis of thiazole-2 $(3 H)$-thiones through [hydroxy(tosyloxy)iodo]benzene, ${ }^{583}$ the HTIB promoted synthesis of 2-substituted 4,5diphenyloxazoles under solvent-free microwave irradiation conditions, ${ }^{584}$ the preparation of oxazoles from ketones and amides using [hydroxy(2,4-dinitrobenzenesulfonyloxy)iodo] benzene, ${ }^{585}$ the one-pot preparation of 2,4,5-trisubstituted oxazoles from ketones, nitriles, and aryliodine(III) triflates generated in situ from iodoarene, $m \mathrm{CPBA}$ and triflic acid, ${ }^{586}$ the preparation of flavones from flavanones using $\mathrm{HTIB},{ }^{587}$ the synthesis of isoflavones from 2'benzoyloxychalcones using polymer-supported HTIB,${ }^{588}$ the preparation of 3tosyloxychromanones by the reaction of HTIB with chromanone and 2-methylchromanone, 589 the HTIB promoted one-pot synthesis of 3-carbomethoxy-4-arylfuran-2-(5H)-ones from ketones, ${ }^{590}$ the HTIB mediated synthesis of 2-aryl-7-cyano(ethoxycarbonyl)-6methylthio- $1 H$-imidazo[1,2-b]pyrazoles from 5-amino-4-cyano(ethoxycarbonyl)-3methylthio- $1 H$-pyrazole and acetophenones, ${ }^{591,592}$ the synthesis of imidazo[2,1-a] isoquinolines using [hydroxy(2,4-dinitrobenzenesulfonyloxy)iodo]benzene, ${ }^{593}$ and the microwave-promoted solvent-free oxidation of $\alpha$-methylene ketones to $\alpha$-diketones. ${ }^{594}$

Recent modifications of this procedure (Scheme 87) include the use of solvent-free reaction conditions, $, 563,575$ application of ionic liquids as solvents, ${ }^{595-597}$ the use of recyclable reagents $\mathbf{2 6 7 - 2 7 1}, 103,260,261,264$ the use of heterocycle-based reagents $\mathbf{2 6 4}$ and $\mathbf{2 6 5}, 560$ and the catalytic $\alpha$-oxytosylation of ketones using $m \mathrm{CPBA}$ as stoichiometric oxidant and iodoarenes as catalysts in the presence of $p$-toluenesulfonic acid. ${ }^{598-601}$

HTIB has been used in various oxidative rearrangements and fragmentations. Justik and Koser have reported a study of an oxidative rearrangement that occurs upon the treatment of arylalkenes $\mathbf{2 7 2}$ with HTIB in 95\% methanol affording the corresponding $\alpha$-aryl ketones $\mathbf{2 7 3}$ in generally high yields (Scheme 88). This oxidative rearrangement is general for acyclic and 
cyclic arylalkenes and permits the regioselective syntheses of isomeric $\alpha$-phenyl ketone pairs. 602

A similar HTIB induced oxidative rearrangement has recently been utilized in the regioselective synthesis of 6-prenylpolyhydroxyisoflavone (wighteone) ( $^{603}$ and in a diastereoselective total synthesis of $( \pm)$-indatraline. ${ }^{604}$ In particular, the key intermediate product $\mathbf{2 7 5}$ in the synthesis of wighteone was prepared by the oxidative rearrangement of $3^{\prime}$ iodotetraalkoxychalcone $\mathbf{2 7 4}, 603$ and the key step in the synthesis of $( \pm$ )-indatraline involved the HTIB promoted diastereoselective ring contraction of a 1,2-dihydronaphthalene $\mathbf{2 7 6}$ to construct the indane ring system 277 (Scheme 89). ${ }^{604}$ A similar oxidative rearrangement of 3cinnamoyl-4-hydroxy-6-methyl-2H-pyran-2-ones with HTIB in dichloromethane followed by cyclization was used by Prakash and co-workers for the direct conversion of $o$ hydroxychalcones into isoflavone derivatives. 605

The HTIB induced oxidative rearrangement of alkenes can be effectively used in ring expansion reactions. Justik and Koser have investigated the oxidative ring expansions of alkylidenebenzocycloalkenes 278 to $\beta$-benzocycloalkenones 279 using HTIB in $95 \%$ methanol (Scheme 90). ${ }^{606}$ This reaction allows the efficient conversion of alkenes $\mathbf{2 7 8}$, which can be conveniently prepared from the respective $\alpha$-benzocycloalkenones by Wittig olefination, to the homologous $\beta$-benzocycloalkenones 279 containing six, seven and eight-membered rings.

Silva and co-workers reported a similar HTIB-promoted ring expansion of 1-vinylcycloalkanol derivatives leading to seven- or eight-membered rings. In a specific example, the reaction of the unsaturated TMS ether $\mathbf{2 8 0}$ with excess HTIB affords benzocycloheptanone derivative 281 in high yield (Scheme 91). ${ }^{607}$

HTIB is commonly used for the oxidative functionalization of arenes, alkenes and alkynes. Koser, Telu and Laali investigated the oxidative substitution reactions of polycyclic aromatic hydrocarbons with iodine(III) sulfonate reagents. ${ }^{608}$ Various polycyclic arenes, such as pyrene, anthracene, phenanthrene, perylene and others, undergo regioselective oxidative substitution reactions with iodine(III) sulfonate reagents in dichloromethane at room temperature to give the corresponding aryl sulfonate esters in moderate to good yields. The reaction of polycyclic aromatic hydrocarbons with HTIB in the presence of trimethylsilyl isothiocyanate leads to the regioselective thiocyanation of the $\mathrm{PAH}$ nucleus, as illustrated by the reaction of anthracene shown in Scheme 92.608

Dihydropyridone derivatives $\mathbf{2 8 2}$ can be efficiently iodinated to afford products $\mathbf{2 8 3}$ by the treatment with $\mathrm{N}$-iodosuccinimide (NIS) in the presence of HTIB (Scheme 93). ${ }^{609}$

Poly[4-(hydroxy)(tosyloxy)iodo]styrene can be used in the halotosyloxylation reaction of alkynes with iodine or $N$-bromosuccinimide (NBS) or $N$-chlorosuccinimide (NCS) (Scheme 94). ${ }^{610}$ The polymer reagent can be regenerated and reused.

HTIB can also be used in the oxidative rearrangements and fragmentations of various nitrogencontaining compounds. Similar to [bis(trifluoroacetoxy)iodo]benzene, HTIB can be applied in the intramolecular cyclization reactions involving $N$-acylnitrenium intermediates 142 (see Scheme 48 in Section 3.4.5). ${ }^{366,611}$ For example, spirodienones 285 bearing the 1-azaspiro [4.5]decane ring system were synthesized from $N$-methoxy-3-(4-halophenyl)propanamides 284 via the intramolecular ipso-cyclization of a nitrenium ion generated with HTIB in trifluoroethanol (Scheme 95). ${ }^{611}$ The HTIB-promoted cyclizations of the appropriate amides were also utilized in the preparation of 2,1-benzothiazine derivatives from sulfonamides 612 and in the synthesis of (-)-lapatin B via oxidative cyclization of $N, N$-diacetylglyantrypine. ${ }^{613}$ 
Similar to [bis(acyloxy)iodo]arenes (see Section 3.4.5), HTIB can serve as excellent oxidant in Hofmann-type degradation of carboxamides to the respective amines. ${ }^{614-616} \mathrm{In}$ a recent example, primary alkyl- and benzylcarboxamides were converted to the corresponding alkylammonium tosylates with poly[4-hydroxy(tosyloxy)iodo]styrene in acetonitrile at reflux in yields ranging from $60 \%$ to $90 \%{ }^{617}$ Likewise, the recyclable reagents $\mathbf{2 6 7}{ }^{103}$ and $\mathbf{2 6 8}{ }^{260}$ (see Section 3.5.1) have been used to convert $p$-nitrobenzamide $\mathbf{2 8 6}$ and phenylacetamide $\mathbf{2 8 8}$ to the respective aniline $\mathbf{2 8 7}$ and benzylammonium tosylate $\mathbf{2 8 9}$ in good yields under mild reaction conditions (Scheme 96). ${ }^{103,260}$

Benzylic alcohols can be oxidized with HTIB under solvent-free microwave irradiation conditions to afford the corresponding aldehydes or ketones in excellent yields. ${ }^{618}$ The glucal derivative 290 was oxidized to the enone $\mathbf{2 9 1}$ by treatment with HTIB in acetonitrile (Scheme 97). ${ }^{619}$

Aryl ketones 292 can be converted to the corresponding substituted benzoic acids 293 by sequential treatment with [hydroxy(2,4-dinitrobenzenesulfonyloxy)iodo]benzene and ureahydrogen peroxide in $[\mathrm{bmim}] \mathrm{BF}_{4}$ ionic liquid (Scheme 98). ${ }^{620}$

Yan and co-workers reported a catalyst- and base-free Suzuki-type coupling reaction of sodium tetraphenylborate with HTIB or other $\lambda^{3}$-iodanes. This non-catalytic coupling affords the respective biaryls in good yields in water solution or solvent-free under microwave irradiation. $621-623$

HTIB and other sulfonate derivatives of iodosylbenzene have also found wide application for the preparation of various iodonium salts.

\subsection{Nitrogen Substituted $\lambda^{3}$-lodanes}

The noncyclic aryliodine(III) derivatives with an iodine nitrogen bond usually lack stability and, with a few exceptions, cannot be isolated as individual compounds. The chemistry of these compounds was discussed in our previous reviews. ${ }^{5,6}$ In particular, several examples of aryliodine(III) amides, ArI(NHCOR) 2 , derived from phthalimide, succinimide, glutarimide, and saccharine have been reported by Varvoglis and co-workers. ${ }^{624-626}$ Aryliodine(III) amides ArI(NHCOR)OAc and ArI(NHCOR)OTs bearing one N-ligand at iodine are plausible intermediates in the Hofmann-type degradation of amides with [bis(acyloxy)iodo]arenes or [hydroxy(tosyloxy)iodo]benzene. ${ }^{614}$ In most cases, these intermediates are highly unstable and instantaneously rearrange at room temperature with loss of iodobenzene to give isocyanates.

The noncyclic azidoiodanes, $\mathrm{PhI}\left(\mathrm{N}_{3}\right) \mathrm{X}(\mathrm{X}=\mathrm{OAc}, \mathrm{Cl}$, OTMS, etc. $)$ or $\mathrm{PhI}\left(\mathrm{N}_{3}\right)_{2}$, were proposed as reactive intermediates in the widely used azidation reactions involving the combination of iodosylbenzene or (diacetoxy)iodobenzene with trimethylsilyl azide or sodium azide. ${ }^{5}$

Attempts to isolate these intermediates always resulted in fast decomposition at -25 to $0{ }^{\circ} \mathrm{C}$ with the formation of iodobenzene and dinitrogen; however, low-temperature spectroscopy and the subsequent chemical reactions in situ provided some experimental evidence toward the existence of these species. The final proof for the existence of azidoiodanes was provided by the preparation and the single-crystal X-ray structure determination of stable azidobenziodoxoles. ${ }^{627}$

(Diazidoiodo)benzene, $\mathrm{PhI}\left(\mathrm{N}_{3}\right)_{2}$, generated in situ from $\mathrm{PhIO} / \mathrm{TMSN}_{3}$ has found some practical application as an efficient reagent for the introduction of the azido function into organic molecules. ${ }^{6}$ Magnus and co-workers reported the synthetically useful azidation of triisopropylsilyl enol ethers 294 affording $\beta$-azido adducts 295 and the azidation of $\mathrm{N}, \mathrm{N}$ dimethylarylamines 296 to give $N$-azidomethyl derivatives 297 in excellent yields (Scheme 99). ${ }^{628-630}$ 
More recently, Bols and co-workers have found that the $\mathrm{PhI}(\mathrm{OAc})_{2} / \mathrm{TMSN}_{3}$ system is similar in reactivity to $\mathrm{IN}_{3}$ and can promote high-yield azidations of ethers, aldehydes and benzal acetals at $0{ }^{\circ} \mathrm{C}$ to room temperature in acetonitrile. ${ }^{631}$ For example, the azidation of ethers 298 under these conditions leads to benzylic azides 299, while the aldehydes 300 initially afford the unstable acyl azides 301, which are converted to carbamoyl azides 302 via the Curtius rearrangement upon heating with an excess of $\mathrm{TMSN}_{3}$ (Scheme 100). These azidations proceed through a radical mechanism and involve the initial generation of $\mathrm{PhI}\left(\mathrm{N}_{3}\right)_{2}$. It is essential for the reaction that $\mathrm{TMSN}_{3}$ is added subsequent to the mixture of $\mathrm{PhI}(\mathrm{OAc})_{2}$ and the substrate; mixing of $\mathrm{TMSN}_{3}$ and $\mathrm{PhI}(\mathrm{OAc})_{2}$ before adding the substrate completely fails to produce any azidation products, presumably because the generated intermediate azidoiodane species decompose before the reaction. ${ }^{631}$

Austin and co-workers utilized the $\mathrm{PhI}\left(\mathrm{N}_{3}\right)_{2}$ mediated vicinal diazidation of a double bond in the key step of the total synthesis of ( \pm )-dibromophakellstatin. The key syn-diazide 304 was prepared by the treatment of pyrazinone $\mathbf{3 0 3}$ with the $\mathrm{PhI}(\mathrm{OAc})_{2} / \mathrm{TMSN}_{3}$ system followed by the addition of tetraethylammonium iodide (Scheme 101). ${ }^{632}$ Under these conditions, the initially generated $\mathrm{PhI}\left(\mathrm{N}_{3}\right)_{2}$, further reacts with the iodide anion leading to the in situ formation of the diazido iodate anion, $\left(\mathrm{N}_{3}\right)_{2} \mathrm{I}^{-}, 633$ which serves as the actual azidating species in this reaction.

The interaction of the $\mathrm{PhI}(\mathrm{OAc})_{2} / \mathrm{NaN}_{3}$ system with organic ditellurides can be used for the generation of the organotellurenyl radicals. This reaction has been utilized in the synthesis of organyltellurophosphates 307 by the treatment of diorganyl phosphites 306 and diorganyl ditellurides $\mathbf{3 0 5}$ with (diacetoxyiodo)benzene and sodium azide in dichloromethane at room temperature (Scheme 102). ${ }^{634}$

\subsection{Stabilized Alkyl Substituted $\lambda^{3}$-lodanes}

Alkyl substituted $\lambda^{3}$-iodanes, RIX $_{2}$, in general lack stability and can exist only as short-lived reactive intermediates in the oxidations of alkyliodides. ${ }^{5,6}$ The thermal stability of alkyliodosyl derivatives can be substantially increased by steric or electronic modification of the alkyl moiety preventing decomposition of the molecule by either elimination or nucleophilic substitution pathways. Most commonly such a stabilization is achieved by the introduction of electron-withdrawing substituents, such as fluorine atoms or a sulfonyl group, into the alkyl moiety. Especially well-investigated and important representatives of stabilized alkyl substituted $\lambda^{3}$-iodanes are [bis(trifluoroacetoxy)iodo]perfluoroalkanes 308,44,417,635-639 [hydroxy(sulfonyloxy)iodo]perfluoroalkanes 309,640,641 1-[bis(trifluoroacetoxy)iodo]- $1 H$, $1 H$-perfluoroalkanes 310, ${ }^{642} 1$-[hydroxy(sulfonyloxy)iodo]- $1 H, 1 H$-perfluoroalkanes 311, 643 , 644 [bis(trifluoroacetoxy)iodo](arylsulfonyl)methane derivatives 312,645 and fluoroalkyliododichlorides 313. ${ }^{225}$ 


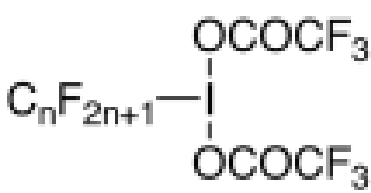

$n=2-12$

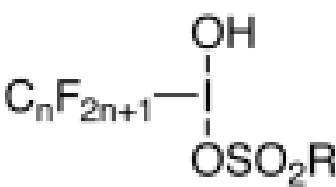

$\mathrm{n}=2-8 ; \mathrm{R}=p-\mathrm{Tol}, \mathrm{CF}_{3}, \mathrm{Me}$

309

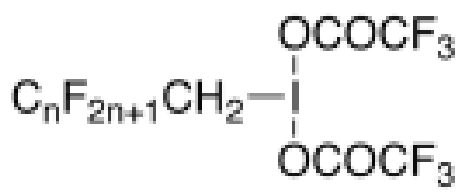

$\mathrm{n}=1-3,7$
308<smiles>O=S(=O)(O[Na])OC(O)CC(F)(F)F</smiles>

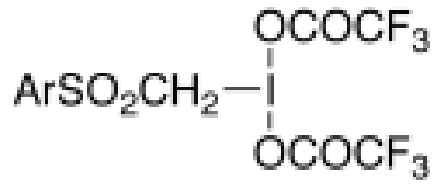

$\mathrm{Ar}=\mathrm{Ph}$ or $p$-Tol

310

$$
\mathrm{n}=1 \text { or } 2 ; \mathrm{R}=p-\mathrm{Tol}, \mathrm{CF}_{3}, \mathrm{Me}
$$

311
312<smiles>[R]CI(Cl)Cl</smiles>

$\mathrm{R}_{\mathrm{f}}=\mathrm{CF}_{3}$ or $\mathrm{H}\left(\mathrm{CF}_{2}\right)_{6}$

The trifluoroacetate derivatives $\mathbf{3 0 8}, \mathbf{3 1 0}$, and $\mathbf{3 1 2}$ are usually prepared by the oxidation of appropriate iodides with $80 \%$ hydrogen peroxide and trifluoroacetic anhydride followed by removal of the volatile products in vacuum (yield 97-98\%) ${ }^{637,638,640} \mathrm{~A}$ convenient procedure for the preparation of [bis(trifluoroacetoxy)iodo]perfluoroalkanes $\mathbf{3 0 8}$ by the oxidation of commercial perfluoroalkyl iodides using urea-hydrogen peroxide complex in a mixture of trifluoroacetic anhydride and trifluoroacetic acid at -5 to $0{ }^{\circ} \mathrm{C}$ was recently reported. ${ }^{417}$ Trifluoroacetates 308 and 310 can be converted to sulfonates 309 and 311 by treatment with the appropriate sulfonic acid. ${ }^{640,644}$ In contrast to the starting trifluoroacetates $\mathbf{3 0 8}$ and $\mathbf{3 1 0}$, sulfonates $\mathbf{3 0 9}$ and $\mathbf{3 1 1}$ have a substantially higher thermal stability and are not water sensitive; they can be purified by crystallization from acetonitrile, and can be stored for several months in a refrigerator.

Single crystal X-ray diffraction studies of several representatives of stabilized alkyl substituted $\lambda^{3}$-iodanes have previously been reported, namely: trifluoromethyliodine(III) difluoride, $\mathrm{CF}_{3} \mathrm{IF}_{2}$ (see Section 3.2.2), ${ }^{190}$ trifluoromethyliodine(III) dichloride, $\mathrm{CF}_{3} \mathrm{ICl}_{2}, 646$ trifluoromethyliodine(III) chloride fluoride, $\mathrm{CF}_{3} \mathrm{I}(\mathrm{Cl}) \mathrm{F},{ }^{647}$ [bis(trifluoroacetoxy)iodo] trifluoromethane, $\mathrm{CF}_{3} \mathrm{I}\left(\mathrm{OCOCF}_{3}\right)_{2}, 648$ trifluoromethyliodine(III) chloride trifluoroacetate, $\mathrm{CF}_{3} \mathrm{I}(\mathrm{Cl}) \mathrm{OCOCF}_{3},{ }^{649}$ [bis(methoxy)iodo] trifluoromethane, $\mathrm{CF}_{3} \mathrm{I}(\mathrm{OMe})_{2},{ }^{650}$ methoxy (trifluoromethyl)iodine(III) chloride, $\mathrm{CF}_{3} \mathrm{I}(\mathrm{Cl}) \mathrm{OMe},{ }^{650}$ fluoroalkyliododichlorides $\mathbf{3 1 3}$ (see Section 3.3.2), ${ }^{225}$ and the bis(trifluoroacetate) $\mathrm{CF}_{3} \mathrm{CH}_{2} \mathrm{I}\left(\mathrm{OCOCF}_{3}\right)_{2} .{ }^{651}$ In particular, the bis (trifluoroacetate) $\mathrm{CF}_{3} \mathrm{CH}_{2} \mathrm{I}\left(\mathrm{OCOCF}_{3}\right)_{2}$ has a distorted T-shaped coordination similar to other known dicarboxylates, but forms a previously unknown tetrameric array of molecules due to strong intermolecular $\left[\cdots 0\right.$ contacts. ${ }^{651}$

[Bis(trifluoroacetoxy)iodo]perfluoroalkanes $\mathbf{3 0 8}$ are the most practically useful representatives of stabilized alkyl substituted $\lambda^{3}$-iodanes. Trifluoroacetates $\mathbf{3 0 8}$ have found practical application as starting compounds for the preparation of (perfluoroalkyl) aryliodonium salts, which are useful electrophilic perfluoroalkylating reagents. ${ }^{44}$ Recently, Tesevic and Gladysz have demostrated the utility of [bis(trifluoroacetoxy)iodo] perfluoroalkanes $\mathbf{3 0 8}$ with a long fluorous alkyl chain $(n=7-12)$ as convenient recyclable oxidants. ${ }^{637,638}$ Similarly to [bis(trifluoroacetoxy)iodo]benzene and (diacetoxyiodo)benzene (see Section 3.4.6) [bis(trifluoroacetoxy)iodo]perfluoroalkanes can serve as excellent reagents for the oxidation of phenolic substrates. The reduced form of the reagent, the respective 
iodoperfluoroalkane, can be efficiently separated from the reaction mixture using fluorous techniques and reused. In a specific example, reagents $\mathbf{3 0 8}(\mathrm{n}=8,10,12)$ can rapidly oxidize 1,4-hydroquinones $\mathbf{3 1 4}$ to the respective quinones $\mathbf{3 1 5}$ in methanol at room temperature (Scheme 103). Subsequent addition of a fluorous solvent, such as perfluoro (methylcyclohexane), results in a liquid/liquid biphase system. The product quinones $\mathbf{3 1 5}$ are generally isolated in about $95 \%$ yields from the methanol phase, and iodoperfluoroalkanes 316 are isolated in $98-99 \%$ yields from the fluorous phase. The recovered iodoperfluoroalkanes $\mathbf{3 1 6}$ may be reoxidized to the initial reagents $\mathbf{3 0 8}$ in $97 \%$ yield and reused. ${ }^{637}$

Westwell and co-workers investigated the oxidation of hydroxylated stilbenes $\mathbf{3 1 7}$ using [bis (trifluoroacetoxy)iodo]perfluorohexane (Scheme 104) ${ }^{417}$ Instead of the expected products of the phenolic oxidation, diaryl-1,2-dimethoxyethanes $\mathbf{3 1 8}$ as mixtures of diastereoisomers were isolated in moderate yields from this reaction. The perfluorohexyl iodide by-product (bp 140 ${ }^{\circ} \mathrm{C}$ ) could be removed simply by evaporation of the reaction mixture under reduced pressure. 417

[Bis(trifluoroacetoxy)iodo]perfluoroalkanes $\mathbf{3 0 8}(\mathrm{n}=7,8,10,12)$ are effective and easily recyclable reagents for the oxidation of aliphatic and benzylic secondary alcohols $\mathbf{3 1 9}$ to ketones $\mathbf{3 2 0}$ in the presence of aqueous $\mathrm{KBr}$ and the absence of organic or fluorous solvents (Scheme 105). ${ }^{638}$ The reduced form of the reagent, the respective iodoperfluoroalkane $\mathbf{3 1 6}$, can be efficiently isolated from the reaction mixture in $96-98 \%$ yield by adding 3-5 volumes of methanol and separating the resulting fluorous/methanolic liquid/liquid biphase system. The recovered iodoperfluoroalkane $\mathbf{3 1 6}$ can be reoxidized to reagent $\mathbf{3 0 8}$ and reused. ${ }^{638}$

It is noteworthy that the fluorous reagents $\mathbf{3 0 8}$ oxidize secondary alcohols in the presence of bromide ions much more rapidly than other iodine(III) compounds (e.g., iodosylbenzene or DIB) under similar conditions. The higher reactivity may in part be ascribed to the directly bound electron-withdrawing perfluoroalkyl substituent in compounds $\mathbf{3 0 8}$, which enhance its oxidizing strength. ${ }^{638}$

\section{8. lodine(III) Heterocycles}

The most important iodine(III) heterocycles are represented by various derivatives of benziodoxole 321 and benziodazole $322 .{ }^{24}$ The collective name "benziodoxoles" is commonly used for heterocycles $\mathbf{3 2 1}$ with iodine and oxygen incorporated in the five-membered ring and various substituents $X$ attached to iodine. The first derivatives of benziodoxole, 1-hydroxy-1,2benziodoxol-3- $(1 H)$-one $(\mathbf{3 2 1}, \mathrm{X}=\mathrm{OH}, 2 \mathrm{R}=\mathrm{O})^{652}$ and 1-chloro-1,2-benziodoxol-3-(1H)-one (321, $\mathrm{X}=\mathrm{Cl}, 2 \mathrm{R}=\mathrm{O}),{ }^{653}$ were prepared over 100 years ago by oxidation or chlorination of 2iodobenzoic acid. In the mid-1980's, 1-hydroxybenziodoxoles have attracted considerable interest and research activity mainly due to their excellent catalytic activity in the cleavage of reactive phosphate esters. ${ }^{33}$ More recently, various new benziodoxole derivatives were synthesized and their usefulness as reagents for organic synthesis was demonstrated. ${ }^{24}$ In contrast to benziodoxoles, the analogous five-membered iodine-nitrogen heterocycles, benziodazoles 322, have received much less attention and, moreover, their structural assignment in some cases was not reliable. The most important and readily available derivative of benziodazole, 1 -acetoxybenziodazole (322, $\mathrm{X}=\mathrm{OAc}, \mathrm{R}=\mathrm{H}$ ), was first prepared in 1965 by the peracetic oxidation of 2 -iodobenzamide, ${ }^{654}$ and the correct structure of this compound was reported in 1997.655 


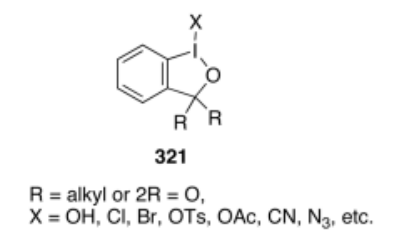

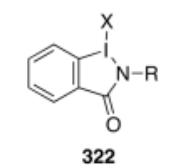

$\mathrm{R}=\mathrm{H}$ or alkyl; $\mathrm{X}=\mathrm{Cl}, \mathrm{OAc}$, etc.

$\mathrm{X}$-ray molecular structures were reported for numerous benziodoxole derivatives $\mathbf{3 2 1}{ }^{100,101 \text {, }}$ $627,656-668$ In general, the five-membered ring in benziodoxole is highly distorted with almost linear alignment of the two electronegative ligands. The I-O bond length in benziodoxolones $(\mathbf{3 2 1}, 2 \mathrm{R}=\mathrm{O})$ varies in a wide range from $2.11 \AA$ in carboxylates $\left(\mathbf{3 2 1}, \mathrm{X}=m-\mathrm{ClC}_{6} \mathrm{H}_{4} \mathrm{CO}_{2}\right)$ 661 to $2.48 \AA$ in the phenyl derivative $(\mathbf{3 2 1}, \mathrm{X}=\mathrm{Ph}),{ }^{100}$ which indicates considerable changes in the ionic character of this bond. The endocyclic C-I-O bond angle is typically around $80^{\circ}$, which is a significant deviation from the expected angle of $90^{\circ}$ for the normal T-shaped geometry of hypervalent iodine. The examples of recently reported X-ray structures of benziodoxoles include phosphoranyl-derived benziodoxoles 323, ${ }^{101}$ 1-bromobenziodoxoles 324, ${ }^{666}$ and 1-trifluoromethylbenziodoxoles 325. ${ }^{667,668}$ Benziodoxoles $\mathbf{3 2 3}$ and $\mathbf{3 2 5}$ were prepared by a standard ligand exchange procedure starting from the appropriate 1acetoxybenziodoxole and a phosphonium ylide or $\mathrm{CF}_{3} \mathrm{SiMe}_{3}$, respectively, ${ }^{101,667,668}$ while 1bromobenziodoxoles 324 were synthesized in $56-60 \%$ yield by oxidative bromination of the appropriate iodoarenes with $N$-bromosuccinimide. ${ }^{666}$<smiles>[R]OC(=O)C(=P)I1OC(=O)c2ccccc21</smiles><smiles>[R]C1([R])OI(Br)c2ccccc21</smiles>

324 $\mathrm{R}=\mathrm{Me}$ or $\mathrm{CF}_{3}$<smiles>[R7]c1ccc2c(c1)C([R])([R])OI2C(F)(F)F</smiles>

325 $\mathrm{R}=\mathrm{Me}$ or $\mathrm{CF}_{3}$ $\mathrm{R}^{1}=\mathrm{H}$ or $\mathrm{Me}$

The structural parameters of benziodazoles ( $\mathbf{3 2 2}, \mathrm{X}=\mathrm{OAc}$ or $\mathrm{Ph})$ in general are similar to those of benziodoxoles. ${ }^{74,102,655}$ The synthesis and structural studies of $\mathrm{N}$-functionalized benziodazoles were recently reported. ${ }^{102} 1$-Acetoxybenziodazoles 327 were prepared by the peracetic oxidation of 2-iodobenzamides $\mathbf{3 2 6}$ derived from alanine or valine (Scheme 106). ${ }^{102}$

The alanine derivative $\mathbf{3 2 8}$ was further converted to phenyliodonium salt $\mathbf{3 2 9}$, which, according to X-ray data, has a pseudo-cyclic structure with an $\mathrm{I} \cdots \mathrm{O}$ distance of $2.56 \AA$ in the benziodoxole ring. ${ }^{102}$ The treatment of pseudo-benziodoxole 329 with sodium bicarbonate affords 1phenylbenziodazole 330 (Scheme 107), whose structural parameters are very similar to the structure of the previously reported 1-phenylbenziodoxole $(321, X=P h)$. In particular, the benziodazole ring system in compound $\mathbf{3 3 0}$ is essentially planar and has a relatively long I-N bond of $2.445 \AA$. This structural study of benziodazole-based phenyliodonium derivatives $\mathbf{3 2 9}$ and $\mathbf{3 3 0}$ provides insight into facile interchange between benziodazole and benziodoxole ring systems under acidic or basic conditions. ${ }^{102}$ 
The distinctive feature of heterocyclic $\lambda^{3}$-iodanes is the considerably higher stability than that of their acyclic analogs. This stabilization is usually explained by the bridging of the apical and the equatorial positions by a five-membered ring, and also by the better overlap of the lone pair electrons on the iodine atom with the p-orbitals of the benzene ring. ${ }^{656,669}$ The greater stability of benziodoxoles enabled the preparation and isolation of otherwise unstable iodine (III) derivatives with I-Br, ${ }^{656,666} \mathrm{I}-\mathrm{OOR},{ }^{670-674} \mathrm{I}-\mathrm{N}_{3}, 627,675,676 \mathrm{I}-\mathrm{CN},{ }^{664,665,677}$ and I$\mathrm{CF}_{3}$ bonds. ${ }^{667,668}$ These various benziodoxole derivatives have found practical application as the reagents for oxidative functionalization of organic substrates. For example, the stable 1azidobenziodoxoles (321, $\mathrm{X}=\mathrm{N}_{3}$ ) can be used as efficient reagents for direct azidation of an unactivated $\mathrm{C}-\mathrm{H}$ bond in alkanes, ${ }^{627,675,676}$ while 1-tert-butylperoxy-1,2-benziodoxol-3 $(1 H)$-one $\left(\mathbf{3 2 1}, \mathrm{X}=\mathrm{OOBu}^{\mathrm{t}}\right)$ is a useful oxidant with numerous synthetic applications. ${ }^{670-674}$ Ochiai and co-workers have recently demonstrated that 1-tert-butylperoxy-1,2-benziodoxol-3 $(1 H)$-one is a particularly useful radical reagent for the generation of $\alpha$-oxy carbon-centered radicals from cyclic ethers and acetals. ${ }^{674,678}$

Togni and co-workers have found that 1-trifluoromethylbenziodoxole $\mathbf{3 3 1}$ is a useful reagent for electrophilic trifluoromethylation of nucleophilic substrates. This reagent, in particular, reacts with $\beta$-ketoesters $\mathbf{3 3 2}$ under mild conditions in the presence of potassium carbonate affording $\alpha$-trifluoromethylated product 333 in good yield (Scheme 108). ${ }^{667,668}$ Likewise, this mild electrophilic trifluoromethylation reagent can be used to transfer a $\mathrm{CF}_{3}$ group to other $\mathrm{C}$ centered nucleophiles, such as $\alpha$-nitro esters, to S-centered nucleophiles, ${ }^{668}$ and secondary or primary aryl- and alkylphosphines. ${ }^{679}$

Very recently, $\mathrm{Hu}$ and co-workers have reported the preparation of the reagent's $\mathbf{3 3 1}$ analog bearing $\mathrm{PhSO}_{2} \mathrm{CF}_{2}$ - substituent on the iodine atom. This new benziodoxole derivative was found to act as the electrophilic (phenylsulfonyl)difluoromethylating reagent for a variety of S-nucleophiles under mild reaction conditions. ${ }^{680}$

\subsection{Iodonium Salts}

Iodonium salts, $\mathrm{R}_{2} \mathrm{I}^{+} \mathrm{X}^{-}$, are defined as positively charged 8-I-2 species with two carbon ligands and a negatively charged counter ion. X-ray structural data for the overwhelming majority of iodonium salts show a significant secondary bonding between the iodine atom and the anion with average bond distances within a range of 2.3 to $2.7 \AA$, which results in a pseudo trigonal bipyramidal geometry similar to $\lambda^{3}$-iodanes with one carbon ligand. In agreement with this model, the experimentally determined bond angle R-I-R in iodonium salts is close to $90^{\circ}$. ${ }^{6}$ The most common and well investigated class of these compounds are diaryliodonium salts, known for over one hundred years and extensively covered in previous reviews. In the 1980s and 1990s significant research activity was focused on aryliodonium derivatives, $\operatorname{Ar}(\mathrm{R}) \mathrm{I}^{+}$ $\mathrm{X}^{-}$, bearing alkynyl-, alkenyl-, or fluoroalkyl groups as ligand $\mathrm{R}$. These aryl substituted iodonium salts are particularly useful reagents for the electrophilic transfer of ligand $\mathrm{R}$ to electron-rich organic substrates. The high reactivity of phenyliodonium salts, $\mathrm{Ph}(\mathrm{R}) \mathrm{I}^{+} \mathrm{X}^{-}$, in these reactions is explained by the "hyperleaving group ability" of the PhI group, which, has a leaving group ability about $10^{6}$ times greater than triflate. ${ }^{681}$

Stable iodonium salts have found numerous practical applications, such as cationic photoinitiators in polymer chemistry, ${ }^{682-685}$ and as biologically active compounds. A summary of the biological properties of iodonium salts is provided in our 1996 review. ${ }^{5}$ In a specific example, a recent study of the in vitro activities of several iodonium salts against oral and dental anaerobes has demonstrated that their activities are comparable to that of chlorhexidine and these compounds may be suitable for incorporation into an oral mouthwash. 686 
In this section, the preparation and chemistry of iodonium salts will be discussed with emphasis on recent synthetic applications.

3.9.1. Alkyl- and Fluoroalkyliodonium Salts-Similar to the alkyl substituted $\lambda^{3}$ iodanes (see Section 3.7), iodonium salts with one or two aliphatic groups generally lack stability. ${ }^{6}$ The presence of electron-withdrawing groups in the alkyl group of iodonium salts has a pronounced stabilizing effect. The most stable derivatives of this type are fluoroalkyl (aryl)iodonium salts $\mathbf{3 3 4 ,} \mathbf{3 3 5}$ and (arylsulfonylmethyl)iodonium triflates $\mathbf{3 3 6}$. The preparation of fluoroalkyl(aryl)iodonium salts and their application as electrophilic fluoroalkylating reagents was reviewed by Umemoto. ${ }^{44}$ Iodonium salts 334-336 are usually prepared by the reaction of the appropriate bis(trifluoroacetates) $\mathbf{3 0 8 ,} \mathbf{3 1 0}$ and $\mathbf{3 1 2}$ (Section 3.7) with benzene in the presence of trifluoromethanesulfonic or other strong acid. ${ }^{6}$ The structure of iodonium triflate $336(\mathrm{Ar}=\mathrm{Tol})$ was unambiguously established by a single-crystal X-ray analysis. ${ }^{645}$
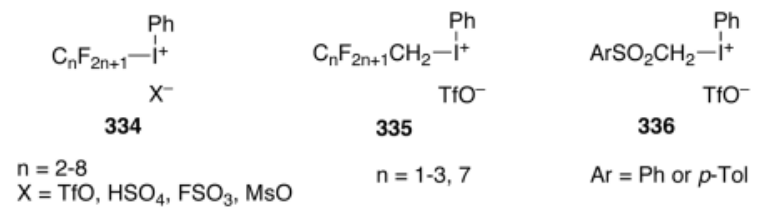

The preparation of fluoroalkyliodonium salts $\mathbf{3 3 7}$ by the reaction of bis(trifluoroacetates) 310 with benzene and triflimide acid was recently reported (Scheme 109). ${ }^{225,651,687}$ The structure of trifluoroethyl(phenyl) iodonium salt $\mathbf{3 3 7}(\mathrm{n}=1)$ was established by a single-crystal $\mathrm{X}$-ray analysis. ${ }^{225}$ In contrast to fluoroalkyliodonium triflates $\mathbf{3 3 5}$, compounds $\mathbf{3 3 7}$ are stable to water and can be used for fluoroalkylations in aqueous media.

Compounds $\mathbf{3 3 7}$ are especially useful as reagents for fluoroalkylation of amino acids and peptides. ${ }^{651,687-691}$ For example, the reaction of iodonium salt $337(n=7)$ with the tert-butyl carboxyl ester of tyrosine $\mathbf{3 3 8}$ in the presence of collidine results in quantitative formation of the monoalkylation product 339 (Scheme 110). ${ }^{687,690}$ Due to this reactivity, iodonium salts 337 can be used as fluorous capping reagents for facile purification of peptides synthesized on the solid phase. ${ }^{687,691}$

3.9.2. Aryl- and Heteroaryliodonium Salts-Diaryliodonium salts belong to the most common and well investigated class of iodine(III) compounds, and the chemistry of these compounds has been extensively covered in previous reviews. ${ }^{5,6}$ In this section, the preparative methods and recent examples of synthetic applications of diaryliodonium and heteroaryliodonium salts, $\mathrm{Ar}_{2} \mathrm{I}^{+} \mathrm{X}^{-}$, are overviewed. Numerous X-ray structures of aryliodonium salts have been reported in the older literature. The more recent structural studies include the X-ray structure reports on (2-methoxy-5-methylphenyl)(4-methoxy-2methylphenyl)iodonium trifluoroacetate, ${ }^{692}$ diaryl zwitterionic iodonium compound $\mathrm{PhI}^{+} \mathrm{C}_{6} \mathrm{H}_{4}-4-\mathrm{SO}_{2} \mathrm{~N}^{-} \mathrm{Tf},{ }^{693}$ 1-naphthylphenyliodonium tetrafluoroborate and 1naphthylphenyliodonium tetrakis(pentafluorophenyl)gallate, ${ }^{694}$ and the study of structural and electronic characteristics of thienyl(aryl)iodonium triflates. ${ }^{695}$

3.9.2.1 Preparation of aryliodonium salts: Diaryliodonium tetrafluoroborates 341 and 343 can be conveniently prepared by the boron-iodine(III) exchange reaction of (diacetoxyiodo) arenes with tetraarylborates $\mathbf{3 4 0}^{696}$ or arylboronic acids $\mathbf{3 4 2}^{697,698}$ followed by the treatment with a saturated sodium tetrafluoroborate solution (Scheme 111). Recent modification of this procedure consists of the treatment of arylrifluoroborates, $\mathrm{ArBF}_{3}{ }^{-} \mathrm{K}^{+}$, with (difluoroiodo) arenes under mild conditions. ${ }^{205}$ Likewise, fluoroorganoiodonium tetrafluoroborates $\left(\mathrm{C}_{6} \mathrm{~F}_{5}\right)_{2} \mathrm{I}^{+} \mathrm{BF}_{4}^{-},\left(4-\mathrm{C}_{5} \mathrm{~F}_{4} \mathrm{~N}\right)_{2} \mathrm{I}^{+} \mathrm{BF}_{4}^{-}$and $\left[\mathrm{C}_{6} \mathrm{~F}_{5}\left(4-\mathrm{C}_{5} \mathrm{~F}_{4} \mathrm{~N}\right) \mathrm{I}^{+} \mathrm{BF}_{4}{ }^{-}\right.$can be prepared by interaction 
of the appropriate (difluoroiodo)arenes with fluorinated organodifluoroboranes, $\mathrm{Ar}_{\mathrm{f}} \mathrm{BF}_{2}$, in dichloromethane at 0 to $20^{\circ} \mathrm{C} .{ }^{178}$

An alternative procedure consists of a similar tin-iodine(III) and silicon-iodine(III) exchange reaction of (diacetoxyiodo)arenes or iodosylbenzene with tetraphenylstannane ${ }^{699}$ or trimethylsilylbenzene ${ }^{699}$ in the presence of boron trifluoride etherate.

Frohn and co-workers reported the preparation of a perfluoroaryliodonium salt, $\left(\mathrm{C}_{6} \mathrm{~F}_{5}\right)_{2} \mathrm{I}^{+}$ $\mathrm{AsF}_{6}{ }^{-}$, by the electrophilic arylation of $\mathrm{C}_{6} \mathrm{~F}_{5} \mathrm{I}$ with a stable pentafluorophenylxenonium hexafluoroarsenate, $\mathrm{C}_{6} \mathrm{~F}_{5} \mathrm{Xe}^{+} \mathrm{AsF}_{6}{ }^{-.700}$

Numerous experimental procedures for the preparation of symmetrical and unsymmetrical diaryl- and hetaryliodonium sulfates and organosulfonates have been reported. ${ }^{3,5,6}$ The most common synthetic approach to unsymmetric diaryl- and hetaryl(aryl)iodonium tosylates is based on the reactions of [hydroxy(tosyloxy)iodo] arenes with arenes, ${ }^{701}$ aryl- or hetaryltrimethylsilanes, ${ }^{702,703}$ aryltributylstannanes, ${ }^{257,704,705}$ or arylboronic acids. ${ }^{706}$ The reaction of HTIB with arylstannanes proceeds under milder conditions compared to arylsilanes and is applicable to a wide range of arenes with electron-withdrawing substituents. Arylboronic acids in general have some advantage over arylstannanes in the case of the electron-rich heterocyclic precursors. ${ }^{706}$

Various unsymmetrically functionalized diaryliodonium triflates 346 can be synthesized by the reaction of iodosylbenzene ${ }^{707}$ or (diacetoxyiodo)arenes $\mathbf{3 4 4 ^ { 7 0 8 }}$ with arenes $\mathbf{3 4 5}$ in trifluoromethanesulfonic acid (Scheme 112). ${ }^{708}$ This simple procedure affords diaryliodonium triflates in relatively high yields, but it is limited to aromatic substrates that are not sensitive to strong acids. Moreover, the formation of the $p$-phenylene type oligomeric iodonium salts as side products may occur upon the reaction of (diacetoxyiodo)benzene with trifluoromethanesulfonic acid. ${ }^{569}$ In a milder and a more selective variation of this procedure (diacetoxyiodo)benzene is reacted with arylboronic acids in the presence of triflic acid at -30 ${ }^{\circ} \mathrm{C}$ to afford aryl(phenyl)iodonium triflates in $74-97 \%$ yields. ${ }^{706}$

Several modified procedures for the preparation of diaryliodonium triflates have recently been reported. Kitamura and Hossain have developed a direct preparation of diaryliodonium triflates in good yields from iodoarenes and aromatic substrates using $\mathrm{K}_{2} \mathrm{~S}_{2} \mathrm{O}_{8}$ as an oxidant in a onepot reaction. ${ }^{709}$ Further modification of this procedure involves the reaction of arenes with elemental iodine and $\mathrm{K}_{2} \mathrm{~S}_{2} \mathrm{O}_{8}$ in trifluoroacetic acid, followed by treatment with sodium triflate (Scheme 113). ${ }^{710,711}$

Olofsson and co-workers have developed a general and efficient one-pot synthesis of symmetrical and unsymmetrical diaryliodonium triflates 349 from both electron-deficient and electron-rich arenes 348 and aryl iodides 347 using $m$ CPBA as the oxidant and triflic acid (Scheme 114). ${ }^{712-714}$ The electron-rich diaryliodonium tosylates are prepared similarly using toluenesulfonic acid instead of triflic acid as the additive. ${ }^{714}$ Symmetrical diaryliodonium triflates can be synthesized by a modified one-pot procedure from iodine, arenes, $m$ CPBA and triflic acid under similar conditions. ${ }^{712,713}$ A similar procedure based on a one-pot reaction of arylboronic acids, aryl iodides, $m \mathrm{CPBA}$ and $\mathrm{BF}_{3} \cdot \mathrm{Et}_{2} \mathrm{O}$ has recently been used for regioselective synthesis of unsymmetrical diaryliodonium tetrafluoroborates. ${ }^{715}$

Skulski and Kraszkiewicz have recently reported a new method for the preparation of various symmetrical diaryliodonium bromides (in 15-88\% crude yields) directly from arenes by the reaction of $\mathrm{ArH}$ with $\mathrm{NaIO}_{4}$ in sulfuric acid followed by the addition of $\mathrm{KBr}{ }^{716}$

A very mild and general method for the preparation of diaryl- and heteroaryliodonium triflates is based on iodonium transfer reactions of iodine(III) cyanides with the respective aryl- or 
heteroarylstannanes. ${ }^{253,255,717,718}$ Specifically, (dicyano)iodonium triflate 350, generated in situ from iodosyl triflate and TMSCN, reacts with tributyltin derivatives of aromatic and heteroaromatic compounds affording the corresponding symmetrical iodonium salts under very mild conditions (Scheme 115). ${ }^{717,718}$

Aryl(cyano)iodonium triflates (e.g. 351) can be used in a similar iodonium exchange with stannylated aromatic precursors affording various mixed diaryl or aryl(heteroaryl) iodonium salts. ${ }^{253,255,695}$ In a recent study, Tykwinski, Hinkle and co-workers have utilized this iodonium transfer reaction in the preparation of a series of mono- and bithienyl(aryl)iodonium triflates $\mathbf{3 5 2}$ with increasingly electron-withdrawing substituents on the aryl moiety (Scheme 116). ${ }^{695}$

The preparation of several macrocyclic iodonium triflates, such as rhomboids $\mathbf{3 5 5}$, a square 358, and a pentagon 359 was recently reported (Scheme 117). ${ }^{719}$ The rhomboid shaped molecules 355 were prepared by the treatment of compounds 353 and 354 with trimethylsilyl triflate. The reaction of dication 356 with compound 357 in the presence of $\mathrm{Me}_{3} \mathrm{SiOTf}$ gave an iodonium containing molecular square 358 in $70 \%$ yield.$^{254,719}$ In addition, a pentagonshaped macrocycle 359 was prepared in $60 \%$ yield from precursors 356 and $\mathbf{3 5 3}$. The structures of these iodonium-containing charged macrocycles were established using elemental analysis, multinuclear NMR and mass spectrometry. These iodonium-containing macromolecules may find potential application in nanotechnology. 719

A very mild and selective approach to aryl- and hetaryliodonium chlorides $\mathbf{3 6 2}$ is based on the reaction of the appropriate aryllithium 360 (generated in situ from bromoarenes and butyllithium) with trans-(chlorovinyl)iodonium dichloride 361 (Scheme 118). ${ }^{720-724}$ The iodonium transfer reagent $\mathbf{3 6 1}$ is prepared by the reaction of iodine trichloride with acetylene in concentrated hydrochloric acid; ${ }^{722}$ this compound is extremely unstable and should be handled and stored with proper safety precautions. ${ }^{721}$ The iodonium transfer procedure with reagent 361 is particularly useful for the preparation of bis(hetaryl)iodonium chlorides 364 from the appropriate nitrogen heterocycles 363 (Scheme 118). ${ }^{721}$

3.9.2.2 Reactions of aryliodonium salts: The most important and synthetically useful reactions of aryliodonium salts include the direct electrophilic arylations of various nucleophiles, the transition metal mediated cross-coupling reactions, and the reactions involving the generation and trapping of the benzyne intermediates.

Numerous examples of the rections of aryliodonium salts with such nucleophiles as thiosulfonate anions, fluoride anion, malonates, and silyl enol ethers under polar, non-catalytic conditions are provided in our previous reviews. ${ }^{5,6}$ In more recent papers, the electrophilic arylations of sodium arenesulfinates, ${ }^{725}$ potassium carbonotrithioates, ${ }^{726}$ and benzazoles ${ }^{727}$ using diaryliodonium salts in ionic liquids, and the arylations of anilines, ${ }^{728}$ sodium tetraphenylborate ${ }^{729}$ and vinylindiums ${ }^{730}$ have been reported.

The mechanism of solvolysis of methoxy-substituted diaryliodonium tetrafluoroborates, $\mathrm{ArI}^{+} \mathrm{Ph}{ }^{-} \mathrm{BF}_{4}$, in methanol and 2,2,2-trifluoroethanol has recently been investigated. ${ }^{731}$ The solvolysis products include alkoxide substitution products (ArOR and $\mathrm{PhOR}$ ) as well as iodoarenes (PhI and ArI). The ratios of products, ArOR/PhOR, range from 8/2 to 4/6. The results of this study provide experimental evidence against the formation of aryl cation under these conditions and support the pathways via ligand coupling or $S_{N} A r 2$ mechanisms involving a solvent molecule as a nucleophile in the transition state. ${ }^{731}$

The reactions of aryliodonium salts with fluoride anion have recently been used for the preparation of fluorine-18 labelled aromatic compounds. $258,705,732$ In a specific example, the ${ }^{18} \mathrm{~F}$ labelled compound $\mathbf{3 6 6}$ was prepared by the reaction of diaryliodonium salt $\mathbf{3 6 5}$ with 
the radioactive ${ }^{18} \mathrm{~F}$ anion (Scheme 119). Compound $\mathbf{3 6 6}$ is used as a positron emission tomography (PET) ligand for imaging peripheral-type benzodiazepine receptor. ${ }^{705}$

Reactions of arylation of carbon nucleophiles using aryliodonium salts are particularly important. Compounds containing an active methylene group, such as malonates, or the respective carbanions formed in situ, react smoothly with diaryliodonium salts to yield $\alpha$ arylated products. ${ }^{733,734}$ Aggarwal and Olofsson have developed a direct asymmetric $\alpha$ arylation of prochiral ketones using chiral lithium amide bases and diaryliodonium salts. ${ }^{721}$ In a specific example, the deprotonation of cyclohexanone derivative 367 using chiral Simpkins' $(R, R)$-base followed by the reaction with pyridyl iodonium salt $\mathbf{3 6 4}$ gave the arylated product 368 in $94 \%$ enantiomeric excess (Scheme 120). This reaction (Scheme 120) has been employed in a short total synthesis of the alkaloid (-)-epibatidine. ${ }^{721}$

Ozanne-Beaudenon and Quideau reported a regioselective dearomatizing phenylation of phenols and naphthols using diaryliodonium salts. ${ }^{735,736}$ For example, the treatment of naphthols 369 substituted at the ortho position by a small electron-donating group with diphenyliodonium chloride leads to their regioselective ortho-phenylation to give products 370 (Scheme 121). The mechanism of this reaction involves a nonradical direct coupling of the ligands on the hypervalent iodine center. ${ }^{735}$ The formation of phenol ethers due to the $\mathrm{O}$ phenylation can also occur when the reaction of phenolate anion with diphenyliodonium chloride is carried out in a polar aprotic solvent such as dimethylformamide. ${ }^{735}$

The O-arylation of the appropriate phenols using symmetrical iodonium salts has been utilized in the synthesis of hydroxylated and methoxylated polybrominated diphenyl ethers, some of which are related to natural products. ${ }^{737,738}$ In particular, several polybrominated diphenyl ethers 373 were prepared by the reaction of iodonium salt 371 with phenols 372 in $N, N$ dimethylacetamide solution under basic conditions (Scheme 122). ${ }^{737}$

Arylations with aryliodonium salts can be effectively catalyzed by transition metals. Aryliodonium salts can serve as efficient reagents in the copper-catalyzed arylation of lithium enolates, ${ }^{739}$ thiophenes, ${ }^{740} 5$-aryl- $2 \mathrm{H}$-tetrazole, ${ }^{741}$ and uracil nucleosides. ${ }^{742}$

Palladium salts and complexes are efficient catalysts in the cross-coupling reactions of diaryliodonium salts with organoboron compounds, ${ }^{743,744}$ organostannanes, ${ }^{745}$ silanes, ${ }^{746}$ organolead triacetates,${ }^{747}$ organobismuth(V) derivatives, ${ }^{748}$ carbon monoxide, ${ }^{749}$ allylic alcohols,${ }^{750}$ functionalized allenes, ${ }^{751,752}$ Grignard reagents, ${ }^{753}$ alkenes, ${ }^{754,755}$ terminal alkynes, ${ }^{756}$ and with arenecarboxylic acids via decarboxylative cross-coupling reaction. ${ }^{757}$ Particularly interesting is the palladium-catalyzed directed C-H activation/phenylation of substituted 2-phenylpyridines and indoles with aryliodonium salts recently reported by Sanford and co-workers. ${ }^{698,758} \mathrm{In}$ a representative example, 2-pyridyl substituted substrates $\mathbf{3 7 4}$ are selectively phenylated to the ortho-position affording products $\mathbf{3 7 5}$ in good yields (Scheme 123). Preliminary mechanistic experiments have provided evidence in support of a rare $\mathrm{Pd}(\mathrm{II}) /$ (IV) catalytic cycle for this transformation. ${ }^{698}$ The preparation of stable triorganyl Pd(IV) complexes by the electrophilic arylation of palladium(II) bipyridine complexes using $\mathrm{Ph}_{2} \mathrm{I}^{+}$ $\mathrm{TfO}^{-}$was reported by Canty and co-workers. ${ }^{759}$

Kitamura and co-workers reported the preparation and uses of several efficient benzyne precursors based on aryliodonium salts. ${ }^{760-764}$ In particular, phenyl[2-(trimethylsilyl)phenyl] iodonium triflate (376) is readily prepared by the reaction of 1,2-bis(trimethylsilyl)benzene with the $\mathrm{PhI}(\mathrm{OAc})_{2} / \mathrm{TfOH}$ reagent system. ${ }^{760}$ The treatment of reagent 376 with tetrabutylammonium fluoride in dichloromethane at room temperature generates benzyne, which can be trapped with a diene to afford the respective benzyne adducts in high yields. ${ }^{760}$ Recent examples of synthetic application of reagent 376 as benzyne precursor include $O$ arylation of carboxylic acids leading to aryl esters $\mathbf{3 7 7}, 765$ preparation of 2-aryl-substituted 
nitriles 379 by arylation of nitriles 378 via a benzyne reaction, ${ }^{766}$ and cycloaddition/elimination reaction of thiophene $S$-oxide $\mathbf{3 8 0}$ with benzyne leading to product $\mathbf{3 8 1}$ (Scheme 124). ${ }^{767}$

Reagent 376 was also used in the synthesis of spiro(imidazolidine-2,3'-benzo[b]thiophene) by a one-pot reaction of benzyne, aryl isothiocyanates and N-heterocyclic carbenes, ${ }^{768}$ and for the preparation of benzo[b]seleno[2,3-b]pyridines by the reaction of acetic acid 2selenoxo- $2 H$-pyridin-1-yl esters with benzyne. ${ }^{769}$

The efficient acylbenzyne precursors, [5-acyl-2-(trimethylsilyl)phenyl]iodonium triflates 382 have recently been prepared by the reaction of the appropriate 1,2-bis(trimethylsilyl) benzenes with the $\mathrm{PhI}(\mathrm{OAc})_{2}$ in the presence of trifluoromethanesulfonic acid in dichloromethane at room temperature. Treatment of these reagents with $\mathrm{Bu}_{4} \mathrm{NF}$ in dichloromethane generates acylbenzynes $\mathbf{3 8 3}$, which can be trapped by furan to give adducts 384 in high yield (Scheme 125). ${ }^{763}$

Lee and co-workers reported the preparation of oxadisilole-substituted benzyne precursors, such as iodonium triflate 386 , from benzobisoxadisilole $\mathbf{3 8 5}$ and the $\mathrm{PhI}(\mathrm{OAc})_{2} / \mathrm{TfOH}$ reagent system. ${ }^{770}$ The treatment of reagent $\mathbf{3 8 6}$ with $\mathrm{Bu}_{4} \mathrm{NF}$ in THF and diisopropylamine at room temperature generates oxadisilole-substituted benzyne $\mathbf{3 8 7}$, which can be trapped with furan to afford adduct $\mathbf{3 8 8}$ in good yield (Scheme 126).

Ko, Kang and co-workers have reported the generation and trapping of 1,2-dehydrocarborane, the carborane analog of benzyne. ${ }^{771}$ The 1,2-dehydrocarborane precursor, phenyl[ $o$ (trimethylsilyl)carboranyl]iodonium acetate, was readily prepared by the reaction of [o(trimethylsilyl)carboranyl]lithium and $\mathrm{PhI}(\mathrm{OAc})_{2}$. 1,2-Dehydrocarborane was efficiently generated from phenyl[o-(trimethylsilyl)carboranyl $]$ iodonium acetate by treatment with $\mathrm{CsF}$ in ether and trapped with dienes such as anthracene, naphthalene, norbornadiene and 2,5dimethylfuran to give the respective 1,2-dehydrocarborane adducts in high yield. ${ }^{771}$

3.9.3. Alkenyliodonium Salts-The chemistry of alkenyliodonium salts was extensively covered in several recent reviews by Ochiai, ${ }^{36,38}$ Okuyama, ${ }^{47,54,55}$ and Zefirov and co-authors. 46 This section of our review will summarize the important recent developments in the preparation and synthetic application of alkenyliodonium salts.

3.9.3.1 Preparation of alkenyliodonium salts: Boron trifluoride-catalyzed silicon-iodine(III) exchange reaction of organosilanes $\mathbf{3 8 9}$ with iodosylarenes followed by treatment with aqueous $\mathrm{NaBF}_{4}$ constitutes the most general method for synthesis of alkenyl(aryl)iodonium tetrafluoroborates 390 (Scheme 127) ${ }^{697,772,773}$ This reaction proceeds under mild conditions and in a stereospecific manner with retention of configuration of organosilanes.

A similar borane-iodine(III) exchange of organoboronic acids 391 with iodosylbenzene or (diacetoxyiodo)benzene in the presence of boron trifluoride etherate is an efficient alternative method for a selective preparation of alkenyl(phenyl)iodonium tetrafluoroborates 392 in excellent yields (Scheme 128). ${ }^{774,775}$

(E)- $\beta$-Fluoroalkenyl(tolyl)iodonium tetrafluoroborates $\mathbf{3 9 3}$ are conveniently synthesized by the treatment of terminal alkynes with 4-iodotoluene difluoride in the presence of boron trifluoride etherate (Scheme 129). ${ }^{206}$ This reaction occurred instantaneously at $-78{ }^{\circ} \mathrm{C}$ to give fluoroalkenyliodonium salts $\mathbf{3 9 3}$ in good yields with high stereoselectivity. Likewise, various alkenyliodonium organosulfonates can be synthesized via electrophilic addition of the appropriate hypervalent iodine reagents to alkynes. ${ }^{184,776,777}$

(E)- $\beta$-Fluoroalkenyl(phenyl)iodonium tetrafluoroborates 395 can be stereoselectively prepared by the reaction of alkynyl(phenyl)iodonium salts 394 with aqueous HF in good yields 
(Scheme 130). ${ }^{778,779}$ The method is applicable to the synthesis of fluoroalkenyliodonium salts having functional groups such as ketone, ester, and chloride.

A very general and mild procedure for the stereospecific synthesis of alkenyliodonium organosulfonates $\mathbf{3 9 8}$ involves the reaction of aryl(cyano)iodonium triflates and tosylates 397 with stannylated alkenes 396 (Scheme 131). ${ }^{780,781}$

The polymer-supported alkenyliodonium tosylates $\mathbf{4 0 1}$ can be prepared by the treatment of polystyrene-based resin 399 with 3-aminocrotonate esters $\mathbf{4 0 0}$ (Scheme 132). ${ }^{782}$ The similar monomeric $\alpha$-acyl- $\beta$-aminoalkenyl(phenyl)iodonium tosylates have been synthesized by the reaction of amino substituted $\alpha, \beta$-unsaturated ketones with [hydroxy(tosyloxy)iodo]benzene. 783

3.9.3.2. Reactions of alkenyliodonium salts: Alkenyl(phenyl)iodonium salts are very reactive compounds because of the excellent leaving group ability of the phenyliodonium moiety ( $10^{12}$ times greater than for iodine itself) combined with its high electron-withdrawing properties (the Hammett substituent constant $\sigma_{\mathrm{m}}$ for the $\mathrm{PhI}^{+}$group is 1.35 ). ${ }^{784}$ Several research groups have recently been involved in the mechanistic studies of nucleophilic substitution in alkenyliodonium salts. ${ }^{785-790}$ Various mechanisms, including $\mathrm{S}_{\mathrm{N}} 1, \mathrm{~S}_{\mathrm{N}} 2$, ligand coupling, and Michael addition-elimination have been observed in these reactions. The mechanistic aspects of the reactions of vinylic iodonium salts with nucleophiles have been reviewed by Okuyama $^{47,791}$ and by Ochiai. ${ }^{36,38}$

Particularly interesting is the recently reported observation of cyclohexyne intermediates $\mathbf{4 0 3}$ as products of $\beta$-elimination in the reactions of 1-cyclohexenyl(phenyl)iodonium salts $\mathbf{4 0 2}$ with mild bases such as tetrabutylammonium acetate, fluoride ion, alkoxides, and amines in aprotic solvents. ${ }^{784,785,792}$ Cyclohexynes $\mathbf{4 0 3}$ could be effectively trapped with tetraphenylcyclopentadienone to give products of [4+2] cycloaddition 404 in high yields (Scheme 133). Cycloheptyne intermediates can be generated under similar conditions from the appropriate iodonium precursors. ${ }^{784,789,793}$

Alkenyl(phenyl)iodonium salts have found synthetic application as alkenylating reagents in the reactions with various nucleophilic substrates. In most cases these reactions proceed with predominant retention of configuration via the addition-elimination mechanism or ligand coupling on the iodine. Recent examples of alkenylations of nucleophiles under non-catalytic conditions include the stereoselective reactions of alkenyliodonium salts with sodium selenide, sodium sulfide, sodium azide, potassium thiocyanate, ${ }^{794}$ and benzotriazole. ${ }^{795}$ In a specific example, functionalized $\beta$-enamines $\mathbf{4 0 5}$ have been prepared by the reaction of polymersupported alkenyliodonium tosylates $\mathbf{4 0 1}$ with various nucleophiles at room temperature (Scheme 134). ${ }^{782}$

$(E)$ - and (Z)-(fluoroalkenyl)boronates $\mathbf{4 0 7}, \mathbf{4 0 9}$ were prepared stereospecifically by the reaction of $(E)$ - or $(Z)$-(2-fluoroalkenyl)iodonium salts $\mathbf{4 0 6 , 4 0 8}$ with di( $p$-fluorophenoxy)alkylboranes, followed by transesterification to pinacol esters (Scheme 135). The mechanism of this reaction involves the initial generation of 2-fluoroalkylideneiodonium ylide by the $\alpha$-deprotonation of iodonium salts with LDA followed by its reaction with with di(p-fluorophenoxy)alkylboranes. 796,797

Only a few examples of non-catalytic alkenylation of carbon nucleophiles are known. In particular, enolate anions derived from various 1,3-dicarbonyl compounds can be vinylated with cyclohexenyl (410) and cyclopentenyl iodonium salts to afford products 411 (Scheme 136). ${ }^{798}$ 
The selectivity of the alkenylation reactions and the yields of products can be dramatically improved by carrying out the reaction of alkenyliodonium salts with carbon nucleophiles in the presence of transition metal compounds in stoichiometric or catalytic amounts. In the presence of a copper(I) catalyst iodonium salts selectively react with iodide anion, ${ }^{778,779}$ organoborates, ${ }^{799}$ Grignard reagents, ${ }^{800}$ and terminal alkynes ${ }^{801}$ to afford the respective crosscoupling products in high yields with complete retention of configuration. A recent example of such a reaction is represented by the copper-mediated cross-coupling of $\mathrm{H}$-phosphonates 413 with vinyliodonium salts $\mathbf{4 1 2}$ leading to 2-arylvinylphosphonates $\mathbf{4 1 4}$ under mild conditions (Scheme 137). ${ }^{802}$

Alkenyliodonium salts can be used as highly reactive reagents for Heck-type olefination, ${ }^{803}$, ${ }^{804}$ Sonogashira-type coupling with alkynes, ${ }^{778,805}$ and similar palladium-catalyzed crosscoupling reactions. ${ }^{206,779,806}$ In a specific example, $(Z)$ - $\beta$-fluoro- $\alpha, \beta$-unsaturated esters 416 were stereoselectively synthesized from (Z)-2-fluoro-1-alkenyliodonium salts $\mathbf{4 1 5}$ by the Pdcatalyzed methoxycarbonylation reaction (Scheme 138). ${ }^{806}$ The reaction proceeded at room temperature and various functional groups on the substrate can tolerate the reaction conditions.

Reactions of alkenyliodonium salts with strong bases may lead to the generation of an alkylidenecarbene via a base-induced $\alpha$-elimination. Alkylidenecarbenes generated by this method can undergo a 1,5-carbon-hydrogen insertion, providing a useful route for the construction of substituted cyclopentenes. ${ }^{807-809}$ In a recent example, an efficient synthesis of fluorocyclopentenes $\mathbf{4 1 8}$ by the reaction of (Z)-(2-fluoroalkenyl)iodonium salts $\mathbf{4 1 7}$ with potassium tert-butoxide has been developed (Scheme 139). The mechanism of this reaction involves the initial generation of ( $\alpha$-fluoroalkylidene)carbenes which give fluorocyclopentenes via $1,5-\mathrm{C}-\mathrm{H}$ insertion. ${ }^{807}$

3.9.4. Alkynyliodonium Salts-The chemistry of alkynyliodonium salts was exhaustively covered in several previous reviews. ${ }^{29,42,810}$ Therefore, this section will only summarize the important recent developments in the preparation and synthetic application of alkynyliodonium salts.

3.9.4.1 Preparation of alkynyliodonium salts: The most common approach to alkynyl (phenyl)iodonium tetrafluoroborates employs the reaction of iodosylbenzene with alkynylsilanes in the presence of boron trifluoride etherate followed by treatment with aqueous $\mathrm{NaBF}_{4} \cdot{ }^{811,812}$ Varvoglis, Koumbis and co-workers have recently used this procedure for the preparation of several ortho-substituted arylethynyl(phenyl)iodonium terafluoroborates $\mathbf{4 2 0}$ from alkynylsilanes $\mathbf{4 1 9}$ (Scheme 140). ${ }^{813}$

A modified procedure for the synthesis of alkynyl(phenyl)iodonium tetrafluoroborates $\mathbf{4 2 2}$ reported by Hara and co-workers consists of the direct reaction of terminal alkynes $\mathbf{4 2 1}$ with iodosylbenzene, $42 \%$ aqueous solution of tetrafluoroboric acid, and a catalytic amount of mercury oxide (Scheme 141). ${ }^{814}$

Yoshida and coauthors have reported a facile preparation of iodonium salts 424 by the reaction of potassium organotrifluoroborates $\mathbf{4 2 3}$ with (difluoroiodo)arenes under mild conditions (Scheme 142). ${ }^{205}$

Alkynyl(phenyl)iodonium tosylates are commonly prepared by gentle heating of [hydroxy (tosyloxy)iodo]benzene with terminal alkynes in chloroform or dichloromethane. ${ }^{812,815,816}$ This method is also applicable to the synthesis of alkynyliodonium mesylates and 4nitrobenzenesulfonates by the reaction of the appropriate [hydroxy(organosulfonyloxy)iodo] benzenes with terminal alkynes under similar conditions. ${ }^{815}$ 
The most versatile method of preparation of alkynyl(phenyl)iodonium triflates 427 employs the iodonium transfer reaction between cyano(phenyl)iodonium triflate $\mathbf{4 2 6}$ and alkynylstannanes $\mathbf{4 2 5}$ under very mild conditions (Scheme 143). ${ }^{817}$ This procedure is particularly useful for the preparation of various complex, functionalized alkynyliodonium derivatives, such as compounds $\mathbf{4 2 8}, \mathbf{4 2 9}, 818 \mathbf{4 3 0}, 819 \mathbf{4 3 1}, 820$ and $\mathbf{4 3 2} .^{821}$ Compounds $\mathbf{4 2 8}-$ 432 are formed under these very mild conditions in high yields (80-90\%) and can be used in subsequent transformations without additional purification.

An alternative general procedure for the selective preparation of alkynyl(phenyl)iodonium triflates in moderate yields employs the reaction of alkynylsilanes or alkynylstannanes with Zefirov's reagent (see Section 3.5.1). ${ }^{813,822}$ This method is also applicable to the synthesis of the parent ethynyl(phenyl)iodonium triflate. ${ }^{823}$

3.9.3.2. Reactions of alkynyliodonium salts: Reactions of alkynyliodonium salts with nucleophiles proceed via an addition-elimination mechanism involving alkylidene carbenes as key intermediates. Depending on the structure of the alkynyliodonium salt, specific reaction conditions, and the nucleophile employed, this process can lead to a substituted alkyne due to the carbene rearrangement, or to a cyclic product via intramolecular 1,5-carbene insertion. ${ }^{42}$ Both of these reaction pathways have been widely utilized in organic synthesis.

Alkynyl(phenyl)iodonium salts have found synthetic application for the preparation of various substituted alkynes by the reaction with the appropriate nucleophiles, such as: enolate anions, 822,824 selenide and telluride anions, ${ }^{825-827}$ dialkylphosphonate anions, ${ }^{828}$ benzotriazolate anion, ${ }^{829}$ imidazolate anion, ${ }^{830} \mathrm{~N}$-functionalized amide anions, ${ }^{831-833}$ and transition metal complexes. ${ }^{834-838}$ Specific recent examples are represented by the preparation of $N$-alkynyl carbamates 435 by alkynylation of carbamates 433 using alkynyliodonium triflates 434 (Scheme 144), ${ }^{832}$ synthesis of ynamides $\mathbf{4 3 7}$ by the alkynylation/desilylation of tosylanilides 436 using trimethylsilylethynyl(phenyl)iodonium triflate (Scheme 145), ${ }^{833}$ and the preparation of $\operatorname{Ir}(\mathrm{III}) \sigma$-acetylide complex 439 by the alkynylation of Vaska's complex 438 (Scheme 146). 834

Alkynyl(phenyl)iodonium salts can be efficiently coupled with organocopper reagents, ${ }^{839}$ or with organoboronic acids or organostannanes in the presence of $\mathrm{Cu}(\mathrm{I})$ catalysts. ${ }^{840,841}$ Specifically, the copper iodide-catalyzed cross- and carbonylative coupling reactions of alkynyliodonium salts 441 with arylboronic acids 440 or organostannanes 443 under mild conditions afford arylacetylenes 442 and aryl alkynyl ketones 444 in high yields (Scheme 147). ${ }^{841}$ Interestingly, alkynyliodonium tetrafluoroborates $\mathbf{4 4 1}$ are more efficient in these coupling reactions than the corresponding iodonium triflates and tosylates.

A variety of five-membered heterocycles can be prepared efficiently by inter- or intramolecular addition/cyclizations of appropriate nucleophiles with alkynyliodonium salts via alkylidene carbene intermediates. ${ }^{29,42,810}$ The intermolecular variant of this cyclization has recently been utilized in the synthesis of 3-substituted-5,6-dihydroimidazo[2,1-b]thiazoles, ${ }^{842} 2$-substituted imidazo[1,2-a]pyrimidines, ${ }^{843}$ and 2-substituted-imidazo[1,2-a]pyridines. ${ }^{844}$ In a specific example, 2-substituted-imidazo[1,2-a]pyridines 447 were synthesized in good yield by cyclocondensation of alkynyl(phenyl)iodonium tosylates 445 with 2-aminopyridine 447 under mild conditions (Scheme 148). The mechanism of this cyclization involves initial nucleophilic addition of the amino group of 2-aminopyridine to the triple bond of the alkynyliodonium salt followed by generation and subsequent cyclization of the intermediate alkylidene carbene. ${ }^{844}$

Ochiai and co-workers have investigated the mechanism for the one-pot synthesis of 2,4disubstituted thiazoles 450 by cyclocondensation of alkynyliodonium salts 448 with thioureas or thioamides 449 (Scheme 149). ${ }^{845}$ This reaction was originally reported by Wipf and 
Venkatraman in $1996 .{ }^{846}$ Ochiai and co-workers have isolated and identified by X-ray analysis intermediate products $\mathbf{4 5 3}$ (as mesylate or tetrafluoroborate salts), which suggests the mechanism involving Michael addition of sulfur nucleophile $\mathbf{4 4 9}$ to alkynyliodonium salt $\mathbf{4 4 8}$ giving intermediate ylide $\mathbf{4 5 1}$ followed by the 1,2-rearrangement of sulfenyl groups in the resulting alkylidene carbene $\mathbf{4 5 2}$ (Scheme 149). ${ }^{845}$

The intramolecular variant of the alkylidene carbene cyclization is achieved by the treatment of functionalized alkynyliodonium salts with the appropriate nucleophile. Recent examples are represented by the preparation of various functionalized 2,5-dihydrofurans by treatment of 3alkoxy-1-alkynyl(phenyl)iodonium triflates with sodium benzenesulfinate, ${ }^{821}$ by the utilization of the alkylidene carbene cyclization in the total syntheses of natural products agelastatin $\mathrm{A}$ and agelastatin $\mathrm{B},{ }^{819}$ and by the preparation of the tricyclic core of $( \pm)$ halichlorine through the use of an alkynyliodonium salt/alkylidenecarbene/1,5 C-H insertion sequence. ${ }^{820}$ In particular, Wardrop and Fritz have utilized the sodium benzenesulfinate induced cyclization of the generated in situ alkynyliodonium triflate $\mathbf{4 5 4}$ leading to dihydrofuran $\mathbf{4 5 5}$ (Scheme 15), which is a key intermediate product in the total synthesis of $( \pm)$-magnofargesin. 821

Feldman and co-workers have applied the sodium $p$-toluenesulfinate induced cyclizations of alkynyliodonium salts $\mathbf{4 5 6}$ and $\mathbf{4 3 1}$ for the preparation of compounds $\mathbf{4 5 7}$ and $\mathbf{4 5 8}$ (Scheme $151)$, the key intermediates in the total syntheses of agelastatins ${ }^{819}$ and ( \pm )-halichlorine, respectively. ${ }^{820}$

\subsection{Iodonium Ylides}

The first preparation of an iodonium ylide by the reaction of dimedone and (difluoroiodo) benzene was reported by Neiland and co-workers in $1957 .{ }^{847}$ Since then a large number of stable iodonium ylides have been prepared, and many synthetic applications have emerged. The chemistry of iodonium ylides was overviewed in several reviews devoted to the reactions of carbenes. ${ }^{56-58}$ This section will summarize the preparation and structural studies of iodonium ylides and important recent developments in their synthetic applications.

3.10.1. Preparation and Structure-The most common and relatively stable structural types of iodonium ylides, namely phenyliodonium bis(organosulfonyl)methides, $\mathrm{PhIC}$ $\left(\mathrm{SO}_{2} \mathrm{R}\right)_{2}$ and the dicarbonyl derivatives $\mathrm{PhIC}(\mathrm{COR})_{2}$, are generally prepared by a reaction of (diacetoxyiodo)benzene with the appropriate disulfone or dicarbonyl compound under basic conditions. ${ }^{848-850}$ The vast majority of iodonium ylides have low thermal stability and can be handled only at low temperature or generated and used in situ. Several structural types of ylides, however, are sufficiently stable for X-ray structural analysis. Single crystal X-ray structural parameters have been reported for 3-phenyliodonio-1,2,4-trioxo-1,2,3,4tetrahydronaphthalenide 459,851 3-phenyliodonio-2,4-dioxo-1,2,3,4-tetrahydro-1oxanaphthalenide $\mathbf{4 6 0}, 851$ mixed phosphonium iodonium ylides $\mathbf{4 6 1} 1^{852}$ and $\mathbf{4 6 2}, 853$ mixed arsonium iodonium ylides $\mathbf{4 6 3}, 854$ cyclic iodonium ylide $\mathbf{4 6 4},{ }^{, 85}$ and phenyliodonium bis (trifluoromethanesulfonyl)methide $\mathbf{4 6 5} .{ }^{856}$ In particular, the X-ray structural analysis for phenyliodonium bis(trifluoromethanesulfonyl)methide $\mathbf{4 6 5}$ shows a geometry typical for an iodonium ylide with the I-C ylide bond length of about $1.9 \AA$ and an C-I-C bond angle of $98^{\circ}$. 856 


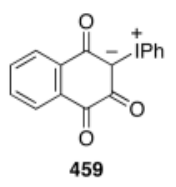

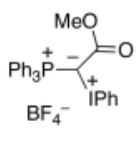

462

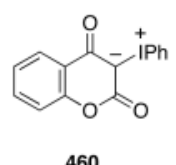

460

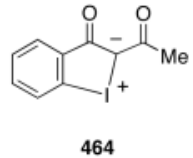

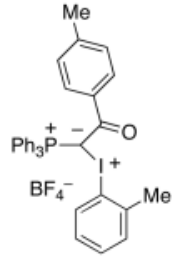

461<smiles>[3H][Te][Te]</smiles>

$\mathrm{Ph}$

465

Ochiai and coworkers have recently reported the intermolecular transylidation reactions between halonium ylides under thermal or catalytic conditions, which allow to synthesize a variety of iodonium ylides 467 (Scheme 152). The transylidations of bromonium 466 to iodonium 467 ylides proceed under thermal conditions and probably involve generation of a reactive carbene intermediate. ${ }^{857}$ The heating of phenyliodonium bis(trifluoromethylsulfonyl) methylide $\mathbf{4 6 5}$ in a large amount of an iodoarene in the presence of 5 mol\% of rhodium(II) acetate as a catalyst results in the transfer of the bis(trifluoromethylsulfonyl)methylidene group to the iodine(I) atom to afford a substituted aryliodonium ylide 467 in a good yield. Reversible nature of the catalytic intermolecular transylidation makes it possible to evaluate the thermodinamic stability of aryliodonium ylides. ${ }^{858}$

A mechanistic study of 1,4 alkyl group migration in hypervalent halonium ylides was recently reported by Moriarty and co-authors. In particular, it was found that the rhodium(II)-acetatecatalyzed decomposion of either 1,3-cyclohexanedione phenyliodonium ylide or 5,5-

dimethyl-1,3-cyclohexanedione phenyliodonium ylide in the presence of alkyl halides yields the corresponding 3-alkoxy-2-halocyclohex-2-enones via a 1,4 alkyl group migration shown to be concerted and intramolecular. ${ }^{859}$

The monocarbonyl iodonium ylides $\mathbf{4 6 9}$ can be quantitatively generated in situ from the (Z)(2-acetoxyvinyl)iodonium salts $\mathbf{4 6 8}$ via an ester exchange reaction with ethoxylithium in THF at $-78{ }^{\circ} \mathrm{C}$ (Scheme 153). ${ }^{1} \mathrm{H}$ NMR measurements indicate that ylides $\mathbf{4 6 9}$ are stable up to -30 ${ }^{\circ} \mathrm{C}$, and they can be conveniently used in the subsequent transformations without isolation. $860-862$

The unstable ylides $\mathrm{PhIC}(\mathrm{H}) \mathrm{NO}_{2}{ }^{863,864}$ and $\mathrm{PhIC}\left(\mathrm{CO}_{2} \mathrm{Me}\right) \mathrm{NO}_{2}{ }^{865,866}$ can be generated in situ from nitromethane and methyl nitroacetate, respectively, and used in the rhodium(II) carbenoid reactions without isolation.

3.10.2. Reactions-Iodonium ylides can serve as convenient precursors to the respective carbene intermediates under thermal, photochemical, or catalytic conditions. A detailed discussion of the reaction mechanisms and synthetic applications of iodonium ylides as carbene precursors can be found in the 2004 review of Muller. ${ }^{58}$

Several new uncatalyzed reactions of iodonium ylides have recently been reported. ${ }^{867-873}$ Koser and co-workers have found that the treatment of electron-rich aromatic substrates, such as anthracene, pyrene, 2-alkylthiophenes, and 1,4-dimethoxybenzene with phenyliodonium bis (carbonyl)methylides in the presence of $\mathrm{BF}_{3} \cdot \mathrm{Et}_{2} \mathrm{O}$ leads to bis(carbonyl)alkylation of the aromatic nucleus ${ }^{867}$ For example, the reactions of 2-alkylthiophenes $\mathbf{4 7 0}$ with ylides $\mathbf{4 7 1}$ afford products $\mathbf{4 7 2}$ in 15-39\% isolated yield (Scheme 154).

The reaction of disulfonyl iodonium ylide $\mathbf{4 7 3}$ with alkyl iodides $\mathbf{4 7 4}$ affords functionalized iodides $\mathbf{4 7 5}$ in moderate yield (Scheme 155). The mechanism of this reaction most likely 
involves the initial transylidation with the formation of unstable alkyliodonium ylides, $\mathrm{RCH}_{2} \mathrm{I}=\mathrm{C}\left(\mathrm{SO}_{2} \mathrm{Ph}\right)_{2}$, which then undergo the intramolecular Stevens rearrangement forming iodides $\mathbf{4 7 5} .868$

Spyroudis and co-workers have reported the reaction of the phenyliodonium ylide of 2hydroxy-1,4-naphthoquinone $\mathbf{4 5 9}$ with amines $\mathbf{4 7 6}$ in refluxing dichloromethane to afford good yields of the indanedione 2-carboxamides 477 (Scheme 156). This reaction proceeds through initial carbene formation, followed by a ring-contraction leading to an intermediate $\alpha, \alpha^{\prime}$ dioxoketene, ${ }^{874}$ which reacts with amines 476 to afford the final amides 477.869 The analogous products are formed when ylide $\mathbf{4 5 9}$ is reacted with amino esters, ureas, amino alcohols, aminophenols, and indole derivatives under thermal conditions. ${ }^{870,871}$

$\mathrm{Li}$ and co-workers have developed a mild and general synthesis of substituted benzofurans by the cycloaddition of iodonium ylides with arynes generated from 2-(trimethylsilyl)aryl triflates and CsF. In a specific example, 2-(trimethylsilyl)aryl triflates $\mathbf{4 7 8}$ smoothly react with iodonium ylides 479 in the presence of $\mathrm{CsF}$ at room temperature giving benzofurans 480 in moderate to good yields (Scheme 157). ${ }^{872}$

Ochiai and co-workers have found that the interaction of monocarbonyl iodonium ylides 482, generated by the ester exchange of (Z)-(2-acetoxyvinyl)iodonium salts 481 with EtOLi, with organoboranes results in the formation of ketones $\mathbf{4 8 4}$, probably via the intermediate formation of the hitherto unknown $\alpha$-boryl ketones $\mathbf{4 8 3}$ (Scheme 158). ${ }^{861}$

The mixed phosphonium-iodonium ylides, such as the tosylate $\mathbf{4 8 5}$, represent a potentially useful class of reagents that combine in one molecule synthetic advantages of a phosphonium ylide and an iodonium salt. ${ }^{854,875-878}$ Specifically, phosphorane-derived phenyliodonium tosylate $\mathbf{4 8 5}$ can react with soft nucleophiles, such as iodide, bromide, benzenesulfinate, and thiophenolate anions, with a selective formation of the respective $\alpha$-functionalized phosphonium ylides 486 (Scheme 159), which can be further converted to alkenes by the Wittig reaction with aldehydes. ${ }^{875,876}$ The analogous arsonium-iodonium ylides (e.g. 463) have a similar reactivity toward nucleophiles. $854,877,879$

The carbenoid reactions of iodonium ylides can be effectively catalyzed by rhodium(II) or copper complexes. ${ }^{56-58}$ The product composition in the rhodium(II) catalyzed reactions of iodonium ylides was found to be identical to that of the corresponding diazo compounds, which indicates that the mechanism of both processes is similar and involves metallocarbenes as key intermediates as it has been unequivocally established for the diazo decomposition. ${ }^{849}$ Recent examples of the transition metal catalyzed carbenoid reactions of iodonium ylides are represented by the following publications: $\mathrm{Rh}(\mathrm{II})$ - or $\mathrm{Cu}(\mathrm{I})$-catalyzed cyclopropanation reactions using the unstable ylides $\mathrm{PhIC}(\mathrm{H}) \mathrm{NO}_{2}{ }^{863}$ and $\mathrm{PhIC}\left(\mathrm{CO}_{2} \mathrm{Me}\right) \mathrm{NO}_{2}{ }^{865,866}$ generated in situ from nitromethane and methyl nitroacetate; $\mathrm{Rh}$ (II)-catalyzed three-component coupling of an ether with a nitromethane-derived carbenoid generated from $\mathrm{PhIC}(\mathrm{H}) \mathrm{NO}_{2} ; 864 \mathrm{Rh}(\mathrm{II})$ - or $\mathrm{Cu}$ (II)-catalyzed insertion of carbene into alkenyl C-H bond in pyrroles, ${ }^{880}$ flavones, ${ }^{881}$ and highly phenylated ethylenes; ${ }^{882} \mathrm{Rh}(\mathrm{II})$-catalyzed reaction of iodonium ylides with conjugated compounds leading to efficient synthesis of dihydrofurans, oxazoles, and dihydrooxepines; 883 synthesis of various heterocycles by $\mathrm{Rh}(\mathrm{II})$-catalyzed reactions of iodonium ylides with vinyl ethers, carbon disulfide, alkynes, and nitriles; $; 84 \mathrm{Rh}(\mathrm{II})$-catalyzed reaction of iodonium ylides with electron-deficient and conjugated alkynes leading to substituted furans, ${ }^{885}$ efficient synthesis of $\beta$-substituted $\alpha$-haloenones by $\mathrm{Rh}$ (II)-catalyzed reactions of iodonium ylides with benzyl halides and acid halides; $886 \mathrm{Rh}(\mathrm{II})$ - or $\mathrm{Cu}(\mathrm{II})$-catalyzed generation/rearrangement of onium ylides of allyl and benzyl ethers via iodonium ylides; $; 87$ and $\mathrm{Rh}$ (II)- or $\mathrm{Cu}$ (II)-catalyzed stereoselective cycloaddition of disulfonyl iodonium ylides with alkenes leading to 1,2,3trisubstituted benzocyclopentenes ${ }^{888}$ or functionalized indanes. ${ }^{889-891}$ 
The metal-catalyzed carbenoid decomposition of iodonium ylides can be applied in asymmetric reactions. ${ }^{865,892-894}$ For example, the copper(II)-catalyzed intramolecular C-H insertion of phenyliodonium ylide $\mathbf{4 8 7}$ in the presence of chiral ligands followed by hydrolysis and decarboxylation affords product $\mathbf{4 8 8}$ in moderate yield with up to $72 \%$ ee (Scheme 160). ${ }^{894}$

A palladium-catalyzed coupling reaction of iodonium ylides $\mathbf{4 8 9}$ with aryl boronic acids $\mathbf{4 9 0}$ was reported. The mild reaction conditions and convenient synthetic accessibility of iodonium ylides $\mathbf{4 8 9}$ make this method a valuable tool for the preparation of diversified 3-aryl-4hydroxycoumarins 491 (Scheme 161). ${ }^{895}$

\subsection{Iodonium Imides}

The chemistry of iodonium imides (also known as iminoiodanes) has been reviewed by Dauban and Dodd in 2003. ${ }^{28}$ Aryliodonium imides $\mathbf{4 9 4}$ are best prepared by the reaction of (diacetoxyiodo)arenes $\mathbf{4 9 2}$ with the respective amides $\mathbf{4 9 3}$ under basic conditions (Scheme 162). ${ }^{28,73,222,896-900}$ Most iodonium imides are stable at room temperature but their storage under an inert atmosphere at low temperature is recommended. They are thermally sensitive and some of them are even claimed to be explosive. Violent decomposition frequently occurs at the melting point. ${ }^{28}$

Single-crystal X-ray structural data have been reported for several $\mathrm{N}$-tosyliminoiodanes, namely, $\mathrm{PhI}=\mathrm{NTs},{ }^{222,901} 2,4,6-\mathrm{Me}_{3} \mathrm{C}_{6} \mathrm{H}_{2} \mathrm{I}=\mathrm{NTs},{ }^{222}$ and $2-\mathrm{MeC}_{6} \mathrm{H}_{4} \mathrm{I}=\mathrm{NTs} .{ }^{898}$ Similar to iodosylarenes (see Section 3.1.2), iminoiodanes have a linear polymeric, asymmetrically bridged structure with the T-shaped geometry around the iodine centers. In the case of $\mathrm{PhI}=\mathrm{NTs}$, the monomeric units are bridged by I-N interactions, while in the more sterically hindered 2,4,6- $\mathrm{Me}_{3} \mathrm{C}_{6} \mathrm{H}_{2} \mathrm{I}=\mathrm{NTs}$ the bridging atom is the oxygen of the tosyl group. ${ }^{22}$ Protasiewicz and coworkers have reported the preparation and X-ray structure of highly soluble, ortho-sulfonyl substituted aryliodonium imide $2-\mathrm{Bu}^{\mathrm{t}} \mathrm{SO}_{2} \mathrm{C}_{6} \mathrm{H}_{4} \mathrm{I}=\mathrm{NTs}$, in which the intramolecular secondary $\mathrm{I} \cdots \mathrm{O}$ bond replaces the intermolecular interactions that are typical of the other iminoiodanes. ${ }^{90}$

Aryliodonium imides have found synthetic applications as useful nitrene precursors under thermal or catalytic conditions in amidation and imidation reactions of various organic substrates and in the aziridination of alkenes. ${ }^{28}$ Only a few examples of the reactions of aryliodonium imides in the absence of transition metal catalysts have been published in the recent literature. Che and coworkers have reported the aziridination of alkenes with phenyliodonium imides generated in situ from $\mathrm{N}$-substituted hydrazines $\mathbf{4 9 5}$ and (diacetoxyiodo)benzene under mild conditions (Scheme 163). ${ }^{902}$ This reaction affords aziridines 496 in good to excellent yields (up to 99\%), and conversions. The practicality and simplicity of this C-N bond formation protocol was exemplified by its application to the aziridination of cholesteryl acetate $\mathbf{4 9 7}$ in a stereoselective manner (Scheme 164) ${ }^{902} \mathrm{~A}$ similar reaction of the $\mathrm{PhI}(\mathrm{OAc})_{2} / \mathrm{N}$-substituted hydrazine $\mathbf{4 9 5}$ system has been used in the nitrene mediated metal-free ring expansions of alkylidenecyclopropanes and alkylidenecyclobutanes. 903

Wirth, Desaize and Richardson have published a detailed study of the aziridination of alkenes with the $\mathrm{PhI}(\mathrm{OAc})_{2} / \mathrm{N}$-substituted hydrazine $\mathbf{4 9 5}$ system and, in particular, reported tentative evidence that this reaction (Scheme 163) proceeds through the formation of an aminoiodane that reacts directly with the alkene. ${ }^{904}$ Furthermore, the authors of this publication ${ }^{904}$ have analyzed the requirements to make this reaction catalytic in iodoarene. This reaction requires an oxidant that will oxidize iodoarenes but that does not oxidize alkenes, and it is possible that no such oxidant actually exists. However, a method in which the hypervalent iodine reagent can be recycled without the need for reisolation is possible. ${ }^{904}$ 
The transition metal catalyzed amidation of $\mathrm{C}-\mathrm{H}$ bonds in saturated or unsaturated substrates represents one of the most common reactions of aryliodonium imides. ${ }^{6,28}$ Recent examples of this reaction using $\mathrm{PhI}=\mathrm{NTs}$ as the nitrene precursor are represented by the following publications: the highly efficient $\mathrm{Ru}(\mathrm{II})$ porphyrin catalyzed $\mathrm{C}-\mathrm{H}$ bond amidation of aldehydes, 905 the aromatic $\mathrm{C}-\mathrm{H}$ amidation mediated by a diiron complex, ${ }^{906}$ the $\mathrm{AuCl}_{3}$-catalyzed nitrene insertion into aromatic and benzylic $\mathrm{C}$-H bonds, ${ }^{907}$ the silver-catalyzed intermolecular and intramolecular amidation of $\mathrm{C}-\mathrm{H}$ bond in saturated hydrocarbons, ${ }^{908,909}$ the $\alpha$-amidation of cyclic ethers catalyzed by $\mathrm{Cu}(\mathrm{OTf})_{2}, 910$ the mechanistic study of catalytic intermolecular amination of $\mathrm{C}-\mathrm{H}$ bonds, ${ }^{911}$ the nitrene insertion into the $\mathrm{sp}^{3} \mathrm{C}-\mathrm{H}$ bonds of alkylarenes and cyclic ethers or the $\mathrm{sp}^{2} \mathrm{C}-\mathrm{H}$ bonds of benzene using a copper-homoscorpionate complex,,${ }^{912}$ the $\mathrm{Co}(\mathrm{II})$-catalyzed allylic amidation reactions, ${ }^{913}$ the $\mathrm{Ru}(\mathrm{II})$ porphyrin-catalyzed amidation of aromatic heterocycles, ${ }^{914}$ and the non-heme iron-catalyzed amidation of aromatic substrates. 915 The enantioselective amidation of a $\mathrm{C}-\mathrm{H}$ bond can also be achieved in the presence of the chiral (salen)manganese(III) complexes. For example, the amidation of substrate $\mathbf{4 9 8}$ occurs at the benzylic C-H bond to afford product 499 with good enantioselectivity (Scheme 165). ${ }^{916}$

Aryliodonium imides are efficient nitrene precursors in the transition metal-catalyzed aziridination of alkenes. ${ }^{6,28}$ Particularly important is the application of PhINTs in the asymmetric aziridination of alkenes using copper catalysts with chiral dinitrogen ligands. ${ }^{917-}$

${ }^{924}$ In a specific example, the PhINTs promoted asymmetric aziridination of alkene $\mathbf{5 0 0}$ affords chiral aziridine $\mathbf{5 0 1}$ in over $99 \%$ ee (Scheme 166). ${ }^{921}$

The aziridination and amidation reactions of aryliodonium imides can be efficiently catalyzed by the $\mathrm{Rh}(\mathrm{II})$ complexes. ${ }^{925-930}$ Dirhodium(II) tetrakis[ $N$-tetrafluorophthaloyl-(S)-tertleucinate], $\mathrm{Rh}_{2}(S \text {-TFPTTL })_{4}$, is an exceptionally efficient catalyst for enantioselective aminations of silyl enol ethers $\mathbf{5 0 2}$ with iodonium imide $\mathbf{5 0 3}$ providing $\alpha$-amido ketones 504 in high yields and with enantioselectivities of up to $95 \%$ ee (Scheme 167). The effectiveness of this catalytic protocol has been demonstrated by an asymmetric formal synthesis of (-)metazocine. ${ }^{925}$ The same catalyst has also been used for the asymmetric synthesis of phenylglycine derivatives by enantioselective amidation of silylketene acetals with aryliodonium imides. ${ }^{926}$

Sanford and co-workers have recently reported the carbon-nitrogen bond-forming reactions of palladacycles with aryliodonium imides. ${ }^{931}$ In particular, palladium(II) complexes (e.g. 505) containing bidentate cyclometalated chelating ligands react with PhINTs at room temperature to insert the tosylimino group into the Pd-C bond (Scheme 168). This tosylimino insertion reaction has been applied to palladacyclic complexes of azobenzene, benzo[h]quinoline, and 8 -ethylquinoline. The newly aminated organic ligands can be liberated from the metal center by protonolysis with a strong acid..$^{931}$

The imido group can be efficiently transferred to the sulfur atom in organic sulfides or sulfoxides, ${ }^{932-935}$ or the nitrogen atom in aromatic nitrogen heterocycles using aryliodonium imides in the presence of copper, ruthenium, or iron complexes. ${ }^{936,937}$ Specific examples are represented by the selective $\mathrm{N}$-imidation of aromatic nitrogen heterocycles (e.g. 506) catalyzed by carbonyl $[$ meso-tetrakis(p-tolyl)porphyrinato]ruthenium(II) $[\mathrm{Ru}(\mathrm{II})(\mathrm{TPP})(\mathrm{CO})]$ (Scheme 169), ${ }^{936}$ and the iron-catalyzed imination of sulfoxides (e.g. 507) and sulfides (Scheme 170). 932

\section{4. lodine(V) Compounds}

The chemistry of organic iodine(V) compounds, or $\lambda^{5}$-iodanes according to the IUPAC nomenclature, in general has been less developed in comparison with the $\lambda^{3}$-iodanes. ${ }^{6}$ The first comprehensive review on the synthetic applications of hypervalent iodine $(\mathrm{V})$ reagents has appeared in $2006,{ }^{22}$ and a specialized review on iodoxybenzoic acid (IBX) was published by 
Wirth in $2001 .{ }^{938}$ There has been a very significant recent interest in the cyclic $\lambda^{5}$-iodanes, mainly IBX and Dess-Martin periodinane (DMP), which have found broad practical application as mild and selective reagents for the oxidation of alcohols and some other useful oxidative transformations. ${ }^{938}$ Despite their importance, IBX and DMP are not perfect reagents and have some disadvantages. IBX is potentially explosive and is insoluble in common organic solvents due to the strong intermolecular secondary bonding creating a three-dimensional polymeric structure, while DMP is highly sensitive to moisture. Several IBX derivatives and analogs with improved properties have been developed in the last 5-6 years and utilized in organic synthesis. In particular, the highly soluble and non-explosive pseudo-cyclic derivatives of IBX, as well as their polymer-supported analogs, have been introduced. This section of our review will summarize the preparation and structure of $\lambda^{5}$-iodanes and overview important recent developments in their synthetic applications.

\subsection{Non-Cyclic and Pseudocyclic lodylarenes}

Iodylarenes, $\mathrm{ArIO}_{2}$, which are also known as iodoxy compounds, are commonly prepared by direct oxidation of iodoarenes with strong oxidants or by disproportionation of iodosylarenes. It is assumed that the initial oxidation of ArI usually leads to iodosylarenes, ArIO, which then slowly disproportionate to $\mathrm{ArI}$ and $\mathrm{ArIO}_{2}$ upon gentle heating, or even at room temperature. 92,256,939 The most common oxidizing reagents that are used for the preparation of iodylarenes from iodoarenes include sodium hypochlorite, sodium periodate, dimethyldioxirane, and oxone. In particular, Skulski and Kraszkiewicz reported an improved method for the preparation of various iodylarenes $\mathbf{5 0 9}$ from the corresponding iodoarenes $\mathbf{5 0 8}$ using sodium periodate as the oxidant dissolved in boiling $30 \%$ aqueous acetic acid (Scheme 171). ${ }^{939}$ Iodylarenes $\mathbf{5 0 9}$ usually precipitate from the reaction mixture and can be additionally purified by recrystallization from hot water or other solvents. Dry iodylarenes are potentially hazardous compounds, which may explode upon impact, scratching with a spatula, or heating, and therefore should be handled with appropriate precautions.

A new facile methodolology for the preparation of noncyclic iodylarenes using peracetic acid as an oxidant in the presence of catalytic amounts of ruthenium trichloride has recently been reported. ${ }^{529,940}$ This new procedure allows the preparation of several previously unknown iodylarenes $\mathbf{5 0 9}$ bearing strongly electron-withdrawing $\mathrm{CF}_{3}$ groups in the aromatic ring. ${ }^{940}$

Iodylbenzene, $\mathrm{PhIO}_{2}$, has a polymeric structure, which makes it insoluble in the majority of organic solvents, with the exception of DMSO. X-ray crystal structural investigations of $\mathrm{PhIO}_{2}$ revealed infinite polymeric chains with strong $\mathrm{I} \bullet \bullet \mathrm{O}$ secondary intermolecular interactions. ${ }^{941}$ Iodylbenzene and other noncyclic iodylarenes in general have found only very limited practical application due to their low stability and explosive properties. ${ }^{22}$

Aryliodyl derivatives bearing an appropriate substituent in the ortho-position to the iodine are characterized by the presence of a pseudocyclic structural moiety due to a strong intramolecular secondary bonding between the hypervalent iodine center and the oxygen atom in the orthosubstituent. Compared to the non-cyclic aryliodyl derivatives, pseudocyclic iodine(V) compounds have much better solubility, which is explained by a partial disruption of their polymeric nature due to the redirection of secondary bonding. ${ }^{89,91}$

Protasiewicz and co-workers have recently reported the preparation of a soluble orthophosphoryl stabilized aryliodyl derivative 511, which was obtained by the hypochlorite oxidation of the appropriate aryliodide $\mathbf{5 1 0}$ (Scheme 173). ${ }^{92}$ Single crystal X-ray analysis of compound $\mathbf{5 1 1}$ has shown a close contact of the phosphoryl oxygen atom and the iodine $(\mathrm{V})$ atom with a distance of $2.612 \AA$, which is significantly shorter than the I $\cdots \bullet O$ distance of 3.291 $\AA$ determined for the unoxidized aryliodide 510. ${ }^{92}$ 
The previously unknown esters of 2-iodoxybenzoic acid (IBX-esters, 513) were prepared by the hypochlorite oxidation of the readily available esters of 2-iodobenzoic acid $\mathbf{5 1 2}$ (Scheme 174) and isolated in the form of stable microcrystalline solids. ${ }^{95,96}$ This procedure allows for the preparation of products $\mathbf{5 1 3}$ derived from various types of alcohols, such as primary, secondary, and tertiary alcohols, adamantanols, optically active menthols and borneol. X-Ray data on products 513 revealed a pseudo-benziodoxole structure in which the intramolecular $\mathrm{I} \cdots \mathrm{O}$ secondary bonds partially replace the intermolecular $\mathrm{I} \cdots \mathrm{O}$ secondary bonds disrupting the polymeric structure characteristic of $\mathrm{PhIO}_{2}{ }^{941}$ and other previously reported iodylarenes. 96 This structural feature substantially increases the solubility of these compounds in comparison to other iodine $(\mathrm{V})$ reagents and affects their oxidizing reactivity. IBX-esters can oxidize alcohols to the respective aldehydes or ketones in the presence of trifluoroacetic acid or boron trifluoride etherate. ${ }^{96}$ Isopropyl 2-iodoxybenzoate $513\left(\mathrm{R}=\mathrm{Pr}^{\mathrm{i}}\right)$ is a particularly useful reagent for the clean and selective oxidation of organic sulfides to sulfoxides. ${ }^{942}$ This reaction proceeds without over-oxidation to sulfones and is compatible with the presence of the hydroxy group, double bond, phenol ether, benzylic carbon, and various substituted phenyl rings in the molecule of organic sulfide.

Methyl 2-iodoxybenzoate $513(\mathrm{R}=\mathrm{Me})$ can be further converted to the diacetate $\mathbf{5 1 4}$ or a similar bis(trifluoroacetate) derivative by treatment with acetic anhydride or trifluoroacetic anhydride, respectively. Single crystal X-ray diffraction analysis of methyl 2-[(diacetoxy) iodosyl]benzoate 514 revealed a pseudo-benziodoxole structure with three relatively weak intramolecular I $\cdots O$ interactions. The dimethyl and diisopropyl esters of 2-iodoxyisophthalic acid were prepared by oxidation of the respective iodoarenes with dimethyldioxirane. Single crystal X-ray diffraction analysis of diisopropyl 2-iodoxyisophthalate $\mathbf{5 1 5}$ showed intramolecular I $\cdots \bullet \mathrm{O}$ interaction with the carbonyl oxygen of only one of the two carboxylic groups, while NMR spectra in solution indicated equivalency of both ester groups. ${ }^{96}$<smiles>COC(=O)c1ccccc1[Te](=O)(OC(C)=O)OC(C)=O</smiles>

514<smiles></smiles>

515

The amides of 2-iodoxybenzoic acid (IBX-amides, 517) were prepared by the dioxirane oxidation of the appropriate derivatives of 2-iodobenzoic acid $\mathbf{5 1 6}$ (Scheme 175) in the form of stable, microcrystalline solids moderately soluble in dichloromethane and chloroform. ${ }^{94}$ This procedure (Scheme 175) can be used for the preparation of products $\mathbf{5 1 7}$ derived from numerous types of amino compounds, such as esters of $\alpha$-amino acids, esters of $\beta$-amino acids, and $(R)-1$-phenylethylamine. Single crystal $\mathrm{X}$-ray analysis of the phenylalanine derivative (517, $\left.\mathrm{R}=(S)-\mathrm{CH}\left(\mathrm{CH}_{2} \mathrm{Ph}\right) \mathrm{CO}_{2} \mathrm{Me}\right)$ revealed a close intramolecular contact of 2.571 Å between the hypervalent iodine center with the oxygen atom of the amido group within each molecule enforcing a planar geometry of the resulting five-membered ring, a geometry that is analogous to that observed for IBX and other benziodoxoles. ${ }^{94}$

2-Iodoxybenzamides $\mathbf{5 1 7}$ are useful oxidizing reagents towards alcohols with a reactivity pattern similar to IBX. A wide range of primary and secondary alcohols can be oxidized by these reagents to the respective carbonyl compounds in excellent yields under mild conditions 
in chloroform. ${ }^{94,943}$ Oxidative kinetic resolution of racemic sec-phenethyl alcohol using reagents $\mathbf{5 1 7}$ has showed very low enantioselectivity (1-6\% ee). ${ }^{943}$

Lee and co-workers have synthesized the polymer-supported IBX-ester $\mathbf{5 1 8}$ and IBX-amides $\mathbf{5 1 9 , 5 2 0}$ starting from the commercially available hydroxy or amino polystyrene in two steps. 944 The oxidant resins 518-520 were prepared with loadings of $0.65-1.08 \mathrm{mmol} / \mathrm{g}$, and were evaluated with a series of alcohol substrates. The polymer supported IBX-amide 520, exhibited particularly fast and efficient oxidative activities toward a series of alcohols under mild reaction conditions. ${ }^{944}$ IBX-amide resin $\mathbf{5 2 0}$ is also an efficient oxidant for oxidative bromination of activated aromatic compounds using tetraethylammonium bromide. ${ }^{945}$ Linclau and co-workers reported an improved synthesis of a solid-supported IBX-amide resins $\mathbf{5 2 1}$ and $\mathbf{5 2 2}$ using inexpensive and commercially available 2-iodobenzoic acid chloride and Merrifield resin. ${ }^{946}$ Oxidation of a range of alcohols to the corresponding carbonyl compounds can be accomplished using 1.2 equivalents of the resins $\mathbf{5 2 1}$ and $\mathbf{5 2 2}$. Recycling of the resin was also possible with minimal loss of activity after two reoxidations. ${ }^{946}$<smiles>O=C(Oc1ccccc1)c1ccccc1I(=O)=O</smiles>

518<smiles>CC(CNC(=O)c1ccccc1P(=O)([O-])O)C1CCCCC1</smiles><smiles>O=C(Nc1ccccc1)c1ccccc1I(=O)=O</smiles>

519

Amides of 2-iodoxybenzenesulfonic acid $\mathbf{5 2 4}$ were prepared by the dioxirane oxidation of the corresponding 2-iodobenzenesulfamides $\mathbf{5 2 3}$ and isolated as stable, microcrystalline products (Scheme 176) ${ }^{947}$ Single crystal X-ray structures of 2-iodylbenzenesulfonamides 524 reveal a combination of intra- and intermolecular $\mathrm{I} \bullet \bullet \mathrm{O}$ interactions leading to a unique heptacoordinated iodine $(\mathrm{V})$ center in the alanine derivative $524\left(\mathrm{R}=(S)-\mathrm{CH}\left(\mathrm{CH}_{3}\right) \mathrm{CO}_{2} \mathrm{Me}\right) .{ }^{93}$

Likewise, esters of 2-iodoxybenzenesulfonic acid $\mathbf{5 2 6}$ were prepared by the dioxirane oxidation in dichloromethane of the respective monovalent iodine derivatives $\mathbf{5 2 5}$ (Scheme 177). These new pseudocyclic hypervalent iodine reagents can selectively oxidize benzyl alcohols to aldehydes, secondary amines to imines, and sulfides to sulfoxides. ${ }^{948}$

The soluble and stable IBX analogs having pseudo-benziodoxazine structure, $N$-(2iodylphenyl)acylamides (NIPA) $\mathbf{5 2 8}$, were prepared in good yields by the oxidation of 2- 
iodoaniline derivatives $\mathbf{5 2 7}$ with 3,3-dimethyldioxirane under mild conditions (Scheme 178). $\mathrm{X}$-Ray data on compounds $\mathbf{5 2 8}$ revealed a unique pseudo-benziodoxazine structure with intramolecular secondary $\mathrm{I} \cdots \bullet \mathrm{O}(2.647 \AA)$ bonding, which is the first reported example of a six-membered pseudo-cyclic scaffold for iodine(V). NIPA reagents 528 are able to selectively oxidize either alcohols or sulfides, with the reactivity depending largely on the substitution pattern on the amide group adjacent to the iodyl moiety. ${ }^{97}$ The synthesis of chiral NIPA reagents $\mathbf{5 2 9}$ and $\mathbf{5 3 0}$ has been carried out based on inexpensive and readily available $(S)$ proline. ${ }^{949}$ The evaluation of these compounds as stereoselective oxidizing reagents toward a racemic alcohol, meso-diol, and a sulfide was performed and moderate enantioselectivities of $29-41 \%$ were achieved. These preliminary results indicate that the NIPA scaffold is a promising structure for further elaboration of chiral iodine(V) oxidants. ${ }^{949}$

As a further expansion of this work, a polymer-supported version of $N$-(2-iodylphenyl) acylamides (NIPA resin) $\mathbf{5 3 1}$ has been prepared in three simple steps. The synthesis employs commercially available aminomethylated polystyrene and affords resin $\mathbf{5 3 1}$ with good loading of $0.70-0.80 \mathrm{mmol} \mathrm{g}^{-1}$. This convenient, recyclable reagent was shown to effect smooth and efficient oxidation of a broad variety of alcohols. ${ }^{950}$

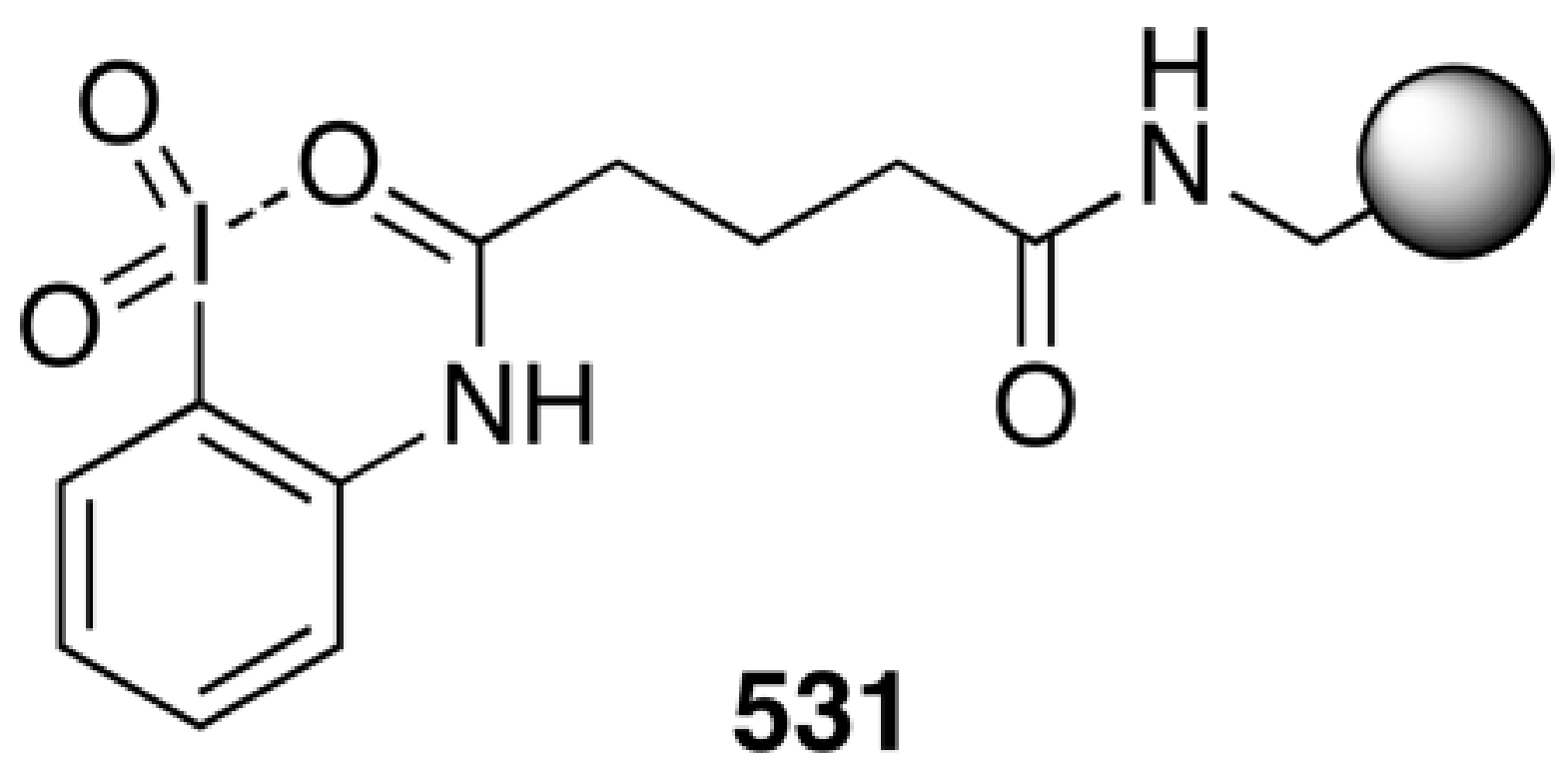

2-Iodylphenol ethers $\mathbf{5 3 3}$ were prepared by the dioxirane oxidation of the corresponding 2iodophenol ethers 532 (Scheme 179) and isolated as chemically stable, microcrystalline products. ${ }^{98}$ Single-crystal X-ray diffraction analysis of 1-iodyl-2-isopropoxybenzene and 1iodyl-2-butoxybenzene revealed pseudo-polymeric arrangements in the solid state formed by intermolecular interactions between the $\mathrm{IO}_{2}$ groups of different molecules. 2-Iodylphenol ethers $\mathbf{5 3 3}$ can selectively oxidize sulfides to sulfoxides and alcohols to the respective aldehydes or ketones. ${ }^{98}$

The polymer-supported analogs of 2-iodylphenol ethers $\mathbf{5 3 4}$ and $\mathbf{5 3 5}$ based on the commercially available aminomethylated polystyrene or Merrifield resin have also been reported. These polymer-supported reagents effect clean and efficient conversion of a wide range of alcohols, including heteroatomic and unsaturated structures, to the corresponding carbonyl compounds. Recycling of the resins is possible with minimal loss of activity after several reoxidations. ${ }^{951}$ 

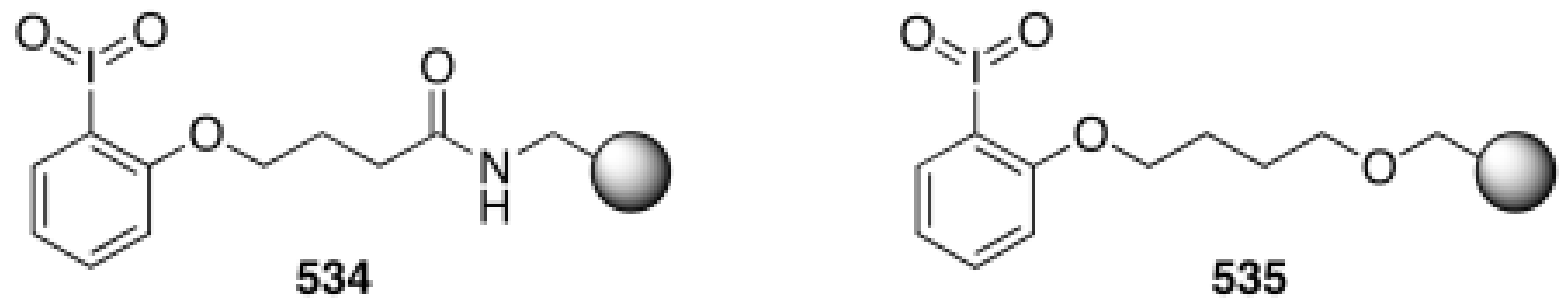

\section{2. lodine(V) Heterocycles}

\subsubsection{2-lodoxybenzoic Acid (IBX) and Analogs}

4.2.1.1. Preparation, structure, and properties: The most important representative of pentavalent iodine heterocycles, 2-iodoxybenzoic acid (IBX, 537), was first prepared in 1893 by Hartman and Mayer. ${ }^{952}$ IBX has the structure of the cyclic benziodoxole oxide (1hydroxy-1-oxo- $1 H$ - $1 \lambda^{5}$-benzo[d][1,2]iodoxol-3-one according to the IUPAC nomenclature), as determined by X-ray structural analysis. ${ }^{107,953,954}$ Most commonly IBX is prepared by the oxidation of 2-iodobenzoic acid with potassium bromate in an aqueous solution of sulfuric acid. ${ }^{955}$ IBX was reported to be explosive under excessive heating or impact, and Dess and Martin attributed the explosive properties of some samples to the presence of bromate impurities. ${ }^{106}$ A convenient procedure for the preparation of IBX $\mathbf{5 3 7}$ which involves oxidation of 2-iodobenzoic acid $\mathbf{5 3 6}$ with oxone (Scheme 180) was reported by Santagostino and coworkers. ${ }^{956}$ This protocol substantially reduced the amount of explosive impurities in the prepared IBX samples.

IBX samples, prepared by the oxidation of 2-iodobenzoic acid with potassium bromate, usually contain a mixture of the powder and the macrocrystalline forms. A detailed X-ray diffraction study of both forms of IBX was published by Stevenson and co-workers. ${ }^{107}$ It was also noticed that the powder form of IBX is more reactive in the reaction with acetic anhydride than the macrocrystalline form and thus is more useful as the Dess-Martin periodinane precursor. Treatment of the macrocrystalline IBX with aqueous sodium hydroxide and then with $\mathrm{HCl}$ can be used to convert it to the more reactive powder form. ${ }^{107}$

The theoretical and experimental study of the $\mathrm{pK}_{\mathrm{a}}$ value and proton affinity of IBX has been published by Williams and co-workers. ${ }^{957}$ Solution-phase acidity determinations were performed in both aqueous media and DMSO. In particular, the aqueous $\mathrm{pK}_{\mathrm{a}}$ value of 2.40 for IBX was obtained by using standard potentiometric titration methods. The relatively high acidity of IBX should be taken in consideration while using this important reagent in the oxidation of complex organic molecules. Very recently, O'Hair and co-authors reported the gas phase proton affinities of the anions of IBX $\left(1300 \pm 25 \mathrm{~mol}^{-1}\right)$ and 2-iodosylbenzoic acid $\left(1390 \pm 10 \mathrm{~kJ} \mathrm{~mol}^{-1}\right)$ using mass spectrometry-based experiments. ${ }^{958}$ The experimental results were supported by theoretical calculations, which yielded proton affinities of 1336 and 1392 $\mathrm{kJ} \mathrm{mol}^{-1}$ for IBX ${ }^{-}$and IBA ${ }^{-}$respectively, at the B3LYP/aug-cc-PVDZ level of theory.

A nonexplosive formulation of IBX (SIBX), consisting of IBX, benzoic acid, and isophthalic acid, has been introduced by Quideau and co-workers. ${ }^{959}$ The synthetic utility of SIBX has been demonstrated on the reactions of hydroxylative phenol dearomatization, ${ }^{418,960,961}$ oxidation of sulfides into sulfoxides, ${ }^{962}$ oxidative demethylation of phenolic methyl aryl ethers, 959 and other useful oxidative transformations. ${ }^{959}$

Several analogs of IBX have been reported in the literature. Vinod and co-workers have developed the water-soluble analogs of IBX, $m$-iodoxyphthalic acid (mIBX) $\mathbf{5 3 8}^{963}$ and a similar derivative of terephthalic acid,,${ }^{964}$ which can oxidize benzylic and allylic alcohols to carbonyl compounds in water. Martin and co-workers first introduced bis(trifluoromethyl) 
benziodoxole oxides 539 and $\mathbf{5 4 0}$, which are stable and non-explosive oxidizing reagents soluble in a wide range of organic solvents. ${ }^{106,965}$ Wirth and co-workers have recently reported the preparation of the tetrafluoro IBX derivative (FIBX, 541), which is more soluble and has higher reactivity than its nonfluorinated counterpart. ${ }^{966}$ Moorthy and co-workers have developed $o$-methyl-substituted IBX (Me-IBX, 542), which is the first modified analog of IBX that oxidizes alcohols in common organic solvents at room temperature due to the hypervalent twisting-promoted rate enhancement. ${ }^{967}$<smiles>O=C(O)c1cccc2c1C(=O)O[Te]2(=O)(O)O</smiles>

538<smiles>O=P1(O)OC(C(F)(F)F)(C(F)(F)F)c2ccccc21</smiles>

539<smiles>CC(C)(C)c1ccc2c(c1)C(O)(C(F)(F)F)OP2(=O)O</smiles>

540<smiles>O=C1OP(O)(O)(O)c2c(F)c(F)c(F)c(F)c21</smiles>

541<smiles>COc1cc2c(c([N+](=O)[O-])c1)P(=O)(O)OC2=O</smiles>

542

2-Iodoxybenzenesulfonic acid $\mathbf{5 4 5}$ (in a cyclic tautomeric form of 1-hydroxy-1H-1,2,3benziodoxathiole 1,3,3-trioxide), a thia-analog of IBX and a powerful oxidizing reagent, was prepared by two different pathways: hydrolysis of the methyl ester of 2-iodylbenzenesulfonic acid 543 or direct oxidation of 2-iodobenzenesulfonic acid 544 (Scheme 181). ${ }^{104}$ The resulting 1-hydroxy-1H-1,2,3-benziodoxathiole 1,3,3-trioxide $\mathbf{5 4 5}$ was found to be thermally unstable and highly reactive towards organic solvents. The structure of its reductive decomposition product, 1-hydroxy-1H-1,2,3-benziodoxathiole 3,3-dioxide (the cyclic tautomeric form of 2iodosylbenzenesulfonic acid), was established by single-crystal X-ray diffraction. ${ }^{104}$

Kawashima and co-workers reported the preparation and oxidative properties of aliphatic iodoxole oxide 547, which is the first example of this class of iodine $(\mathrm{V})$ compounds. The tetracoordinate 1,2-iodoxetane $\mathbf{5 4 7}$ was prepared by the fluorination of a tricoordinate 1,2iodoxetane $\mathbf{5 4 6}$ with xenon difluoride followed by hydrolysis (Scheme 182). ${ }^{968}$ Compound $\mathbf{5 4 7}$ oxidizes alcohols and sulfides to the corresponding carbonyl compounds and sulfoxides, respectively, in good yields under mild conditions. ${ }^{968}$

The preparation and oxidative reactivity of several polymer-supported analogs of IBX have been reported. Giannis and Mülbaier have developed the aminopropylsilica gel based reagent 548, which can oxidize various primary and secondary alcohols to the respective carbonyl compounds in excellent yields at room temperature in THF under heterogeneous conditions and can be regenerated by oxidation with oxone without any loss of activity. ${ }^{969}$ Rademann and coworkers prepared the polystyrene based polymeric analog of IBX 549, which was characterized by IR spectroscopy, elemental analysis, and MAS-NMR spectroscopy. ${ }^{970}$ Reagent 549 oxidizes various primary, secondary, benzylic, allylic, terpene alcohols, and the carbamate-protected aminoalcohols to afford the respective aldehydes or ketones in excellent yields, and it can be recycled by repeated oxidation after extensive washings. Lei and coworkers have developed a polymer-supported IBX derivative 550, which has the advantages of a simplified preparation method and a high oxidation activity of $1.5 \mathrm{mmol} \mathrm{g}{ }^{-1} \cdot{ }^{971} \mathrm{~A}$ conceptually different approach was used by Sutherland and co-workers for the preparation of the polystyrene based reagent $\mathbf{5 5 1}$; in this procedure the iodobenzoic acid moiety was introduced directly to the resin backbone by the iodination/oxidation sequence. ${ }^{972}$ Very 
recently, the preparation of functional organic-inorganic colloids modified by IBX $\mathbf{5 5 2}$ has been reported by Hatton and co-workers. ${ }^{973}$

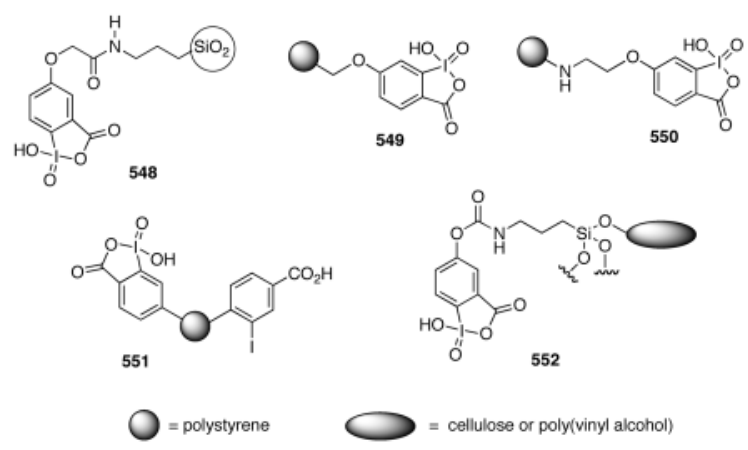

4.2.1.2. Synthetic applications of IBX: IBX has attracted significant interest as a mild and selective oxidizing reagent. IBX is a particularly useful oxidant for the selective oxidation of alcohols to carbonyl compounds, even in complex molecules in the presence of other functional groups. ${ }^{974-976}$ Recently this oxidative methodology has been utilized in numerous syntheses, such as: the total synthesis of (+)-wailupemycin $\mathrm{B},{ }^{977}$ the total synthesis of (-)decarbamoyloxysaxitoxin, ${ }^{978}$ the total synthesis of abyssomicin C and atrop-abyssomicin C, 979 the stereoselective synthesis of pachastrissamine (jaspine B), ${ }^{980}$ the syntheses of $( \pm$ )pterocarpans and isoflavones, ${ }^{981}$ the total synthesis of $( \pm)$-nitidanin, ${ }^{982}$ the total synthesis of lagunamycin, ${ }^{983}$ the synthesis of (-)-agelastatin, ${ }^{984}$ the syntheses of heliannuols B and D, ${ }^{985}$ the synthesis of the C1-C15 fragment of dolabelide C, ${ }^{986}$ the total syntheses of (-)subincanadines $\mathrm{A}$ and $\mathrm{B},{ }^{987}$ the synthesis of the spiro fused $\beta$-lactone- $\gamma$-lactam segment of oxazolomycin, ${ }^{988}$ the synthesis of marine sponge metabolite spiculoic acid A, ${ }^{989}$ the synthesis of optically pure highly functionalized tetrahydro-isoquinolines, ${ }^{990}$ the preparation of Fmocprotected amino aldehydes from the corresponding alcohols, ${ }^{991}$ and the selective oxidation of hydroxyl-substituted organotrifluoroborates to the respective carbonyl compounds. ${ }^{992}$

The synthetic usefulness of IBX in general is significantly restricted by its low solubility in most organic solvents with the exception of DMSO. However, in several recent reports it has been shown that IBX can be used as an effective oxidant in other than DMSO solvents. ${ }^{993-}$ 996 More and Finney have found that primary and secondary alcohols can be oxidized into the corresponding aldehydes or ketones in excellent yields (90-100\%) by heating a mixture of the alcohol and IBX in common organic solvents. ${ }^{993}$ All reaction by-products can be completely removed by filtration. This method was used for the efficient preparation of the ribosyl aldehyde $\mathbf{5 5 3}$ (Scheme 183), the key intermediate in the stereoselective synthesis of the core structure of the polyoxin and nikkomycin antibiotics. ${ }^{994}$

Kuhakarn and co-workers have recently found that IBX can be used for the oxidation of alcohols in a water/dichloromethane 1:1 mixture in the presence of tetrabutylammonium bromide. ${ }^{996}$

IBX is especially useful for the oxidation of 1,2-diols. Moorthy and co-workers have investigated the reactions of IBX with various vicinal diols and found that the oxidative cleavage of the $\mathrm{C}$-C bond, as well as the previously known oxidation to $\alpha$-ketols or $\alpha$-diketones, can occur in these reactions. ${ }^{997}$ In DMSO solutions, IBX oxidatively cleaves strained and sterically hindered syn 1,2-diols, while the non-hindered secondary glycols are oxidized to $\alpha$ ketols or $\alpha$-diketones. The use of trifluoroacetic acid as a solvent leads to efficient oxidative fragmentation of 1,2-diols of all types. ${ }^{997}$ The oxidation of 1,2-diols using IBX in DMSO has been utilized for the synthesis of $\alpha$-ketols ${ }^{977,998,999}$ or $\alpha$-diketones. ${ }^{1000}$ For example, in the 
key step of the total synthesis of the streptomyces maritimus metabolite - wailupemycin B, IBX oxidation led to the desired hydroxyketone $\mathbf{5 5 4}$ without any cleavage of the glycol C-C bond (Scheme 184). 977

An interesting IBX-mediated oxidation of primary alcohols or aldehydes to $\mathrm{N}$ hydroxysuccinimide esters $\mathbf{5 5 5}$ was developed by Giannis and Schulze. ${ }^{1001}$ The generality of this procedure was demonstrated on a variety of aliphatic, allylic, and benzylic alcohols (Scheme 185).

Chen and co-workers reported a mild, efficient, and environmentally benign protocol for the oxidation of alcohols with IBX in the ionic liquid 1-butyl-3-methylimidazolium chloride and water. ${ }^{995}$ Stirring a solution of the alcohol and IBX in 1-butyl-3-methyl-imidazolium chloride followed by removal of water at room temperature and subsequent extraction with ether or ethyl acetate gives excellent yields (88-99\%) of the corresponding carbonyl compounds. No overoxidation to acids was observed in the case of aldehyde products, and various functionalities such as methoxy and nitro groups, double bonds, and a furan ring could be tolerated. The oxidation of glycols under these conditions, depending of the amount of IBX used, affords $\alpha$-ketols or $\alpha$-diketones. ${ }^{995}$

Catalytic IBX-based procedures for the oxidation of alcohols have been reported by Giannis and Schulze, ${ }^{1002}$ Vinod and co-workers, ${ }^{1003}$ and by Page et al. ${ }^{1004}$ In particular, the oxidation of primary or secondary alcohols using catalytic amounts (20-30 mol\%) of IBX or 2iodobenzoic acid (IBA) in the presence of oxone as a stoichiometric oxidant in aqueous acetonitlile at $70{ }^{\circ} \mathrm{C}$ affords the corresponding carboxylic acids or ketones in $74-97 \%$ yield. 1003 A further modification of this procedure employs tetraphenylphosphonium monoperoxysulfate as the oxidant in the presence of catalytic 2-iodobenzoic acid; in this case primary alcohols are oxidized to aldehydes without overoxidation to carboxylic acids. ${ }^{1004}$

IBX in DMF has been shown to be an excellent reagent for the oxidation of various phenols to $o$-quinones. ${ }^{1005}$ This procedure was used for the oxidation of phenol $\mathbf{5 5 6}$ to quinone $\mathbf{5 5 7}$ (Scheme 186), the key intermediate in the total synthesis of a novel cyclooxygenase inhibitor $\left( \pm\right.$ )-aiphanol. ${ }^{1006}$ The same protocol was recently utilized in the synthesis of $( \pm)$-brazilin, a tinctorial compound found in the alcoholic extracts of trees collectively referred to as Brazil wood, by Pettus et al. ${ }^{1007}$

Quideau and co-workers have recently utilized the non-explosive formulation of IBX (SIBX) in the total synthesis of the bissesquiterpene (+)-aquaticol by biomimetic oxidative dearomatization of the appropriate phenolic substrate via an orthoquinol intermediate. ${ }^{961}$

The practical value of IBX as a reagent was recently extended to a variety of other synthetically useful oxidative transformations, such as: the one-step synthesis of $\alpha, \beta$-unsaturated carbonyl systems from saturated alcohols and carbonyl compounds, ${ }^{1008}$ the selective oxidation of the benzylic carbon, ${ }^{1009,1010}$ the oxidation of amines to imines ${ }^{1011,1012}$ and nitriles, ${ }^{1013-1017}$ the oxidative deprotection of dithianes ${ }^{1011}$ and 1,3-oxathiolanes, ${ }^{1018}$ the oxidation of indoles into 3-hydroxyoxindoles and isatins in the presence of $\mathrm{InCl}_{3}$ or $\mathrm{CeCl}_{3}, 1019,1020$ the aromatization of 1,4-dihydropyridines, ${ }^{1021}$ the $\alpha$-hydroxylation of the $\alpha$-alkynyl carbonyl systems leading to the corresponding tertiary alcohols ${ }^{1022}$ or $(Z)$-enediones, ${ }^{1023}$ the synthesis of $\beta$-(hetero)aryl$\alpha$-nitro- $\alpha, \beta$-enals, ${ }^{1024}$ the synthesis of quinoxaline derivatives from 1,2-diketones and $o$ phenylenediamines, ${ }^{1025}$ the oxidative cyclization of anilides and related compounds leading to various heterocyclic systems, ${ }^{1026}$ the generation of alkoxyamidyl radicals from the corresponding acylated alkoxyamines, ${ }^{1027}$ the preparation of nitrile oxides from aldoximes, 1028 and various multicomponent oxidative transformations. ${ }^{1029-1032}$ Several specific examples of these reactions are discussed below. 
Nicolaou and co-workers reported a one-pot procedure for the oxidation of alcohols, ketones, and aldehydes to the corresponding $\alpha, \beta$-unsaturated species using IBX under mild conditions. For example, cycloalkanols $\mathbf{5 5 8}$ react with two equivalents of IBX in a 2:1 mixture of either fluorobenzene or toluene and DMSO at gentle heating to afford the corresponding $\alpha, \beta$ unsaturated ketones $\mathbf{5 5 9}$ in good yields (Scheme 187). ${ }^{1008}$

IBX is an efficient and selective reagent for the oxidation of alkyl substituted aromatic compounds 560 at the benzylic position to the corresponding carbonyl derivatives 561 (Scheme 188). This reaction is quite general and can tolerate a variety of substituents within the aromatic ring. Overoxidation to the corresponding carboxylic acids is not observed even in the presence of electron-rich substituents. ${ }^{1009}$

Similar to the oxidation of alcohols, secondary amines $\mathbf{5 6 2}$ can be oxidized with IBX in DMSO to yield the corresponding imines $\mathbf{5 6 3}$ in good to excellent yields (Scheme 189). ${ }^{1011}$

A variety of new heterocycles $\mathbf{5 6 5}$ can be synthesized by the treatment of unsaturated aryl amides, carbamates, thiocarbamates, and ureas $\mathbf{5 6 4}$ with IBX (Scheme 190). ${ }^{1026,1033}$ The mechanism of this reaction has been investigated in detail. ${ }^{1034}$ On the basis of solvent effects and D-labeling studies, it was proposed that the IBX-mediated cyclization of anilides in THF involves an initial single electron transfer (SET) to a THF-IBX complex followed by deprotonation, radical cyclization, and concluding termination by hydrogen abstraction from THF. ${ }^{1034}$ A similar IBX-mediated cyclization was applied in the synthetic protocol for the stereoselective preparation of amino sugars. ${ }^{1035}$

Studer and Janza reported a method for the generation of alkoxyamidyl radicals starting from the corresponding acylated alkoxyamines using IBX as a single electron transfer (SET) oxidant. Stereoselective 5-exo and 6-exo reactions with these N-heteroatom-centered radicals lead to isoxazolidines and [1,2]oxazinanes (e.g. 566) (Scheme 191). ${ }^{1027}$

IBX has also been used for the preparation of the 3,5-disubstituted isoxazolines 567. SET oxidation of substituted aldoximes with IBX in dichloromethane produces the respective nitrile oxide which then undergoes 1,3-dipolar addition with an alkene component (Scheme 192). ${ }^{1028}$

A one-pot three-component synthesis of $\alpha$-iminonitriles 568 by IBX/tetrabutylammonium bromide-mediated oxidative Strecker reaction (Scheme 193) was reported by Zhu, Masson and co-workers. ${ }^{1032}$ This methodology was applied to a two-step synthesis of indolizidine via a microwave-assisted intramolecular cycloaddition of $\alpha$-iminonitrile.

The IBX-mediated oxidative Ugi-type multicomponent reaction of tetrahydroisoquinoline with isocyanides and carboxylic acids affords the $\mathrm{N}$ and $\mathrm{C} 1$ functionalized tetrahydroisoquinolines 569 in good to excellent yields. ${ }^{1031}$ Likewise, the three-component Passerini reaction of an alcohol, carboxylic acid, and an isonitrile in the presence of IBX affords the corresponding $\alpha$ acyloxy carboxamides $\mathbf{5 7 0}$ in generally high yields (Scheme 194). ${ }^{1030}$

4.2.2. Dess-Martin Periodinane (DMP)—Dess-Martin periodinane (DMP, 572) was originally introduced in $1984^{1036}$ and since then has emerged as the reagent of choice for the oxidation of primary and secondary alcohols to aldehydes and ketones, respectively. ${ }^{22,59} \mathrm{DMP}$ is best prepared by the reaction of IBX $\mathbf{5 7 1}$ with acetic anhydride in the presence of $p$ toluenesulfonic acid (Scheme 195). ${ }^{1037}$

Due to the mild reaction conditions (room temperature, absence of acidic or basic additives) and high chemoselectivity, DMP is especially suitable for the oxidation of alcohols containing sensitive functional groups, such as unsaturated moieties, amino groups, silyl ethers, phosphine oxides, sulfides, selenides, etc. In case of epimerization sensitive substrates, DMP allows clean 
oxidation with virtually no loss of enantiomeric excess. Thus, the oxidation of $\mathrm{N}$-protected $\beta$ amino alcohols with DMP afforded the respective aldehydes with 99\% ee and excellent chemical yields, while Swern oxidation gave unsatisfactory results (50-68\% ee). ${ }^{1038}$ The DMP oxidation is accelerated by the addition of water to the reaction mixture immediately before or during the reaction. ${ }^{1039}$ Silyl ethers can be effectively used instead of alcohols in the DMP oxidations affording the corresponding carbonyl compounds in excellent yields. ${ }^{1040}$ The DMP oxidation of 1,2-diols generally cleaves the glycol C-C bond as illustrated by the synthesis of tricyclic enol ether $\mathbf{5 7 4}$ from diol $\mathbf{5 7 3}$ via tandem 1,2-diol cleavage-intramolecular cycloaddition (Scheme 196). ${ }^{1041}$

Because of the unique oxidizing properties and convenience of use, DMP is widely employed in the synthesis of biologically important natural products. Recently DMP has been used in the key oxidation steps of the following synthetic works: the preparation of 2-alkynyl acroleins, 1042 the oxidation of $\alpha$-diazo- $\beta$-hydroxyesters to $\alpha$-diazo- $\beta$-ketoesters, ${ }^{1043}$ the scale-up syntheses of (-)-epicatechin- $(4 \beta, 8)-(+)$-catechin and (-)-epicatechin-3- $O$-galloyl-(4 $\beta, 8)-(-)$ epicatechin-3-O-gallate, ${ }^{1044}$ the synthesis of a potent anti-tumor therapeutic 7-Epi (+)FR900482, 1045 the formal total synthesis of $( \pm)$-platensimycin, ${ }^{1046}$ the total synthesis of several members of the vinca and tacaman classes of indole alkaloids, ${ }^{1047}$ the oxidation of the appropriately functionalized hydroxyporphyrins to chlorin- $\alpha$-diones and bacteriochlorintetraones, ${ }^{1048}$ the synthesis of an $N$-mesityl substituted chiral imidazolium salt, the $\mathrm{N}$ heterocyclic carbene precursor, ${ }^{1049}$ the synthesis of new lavendamycin analogues, ${ }^{1050}$ the synthetic studies towards the total synthesis of providencin, ${ }^{1051}$ the stereo-controlled synthesis of prelasalocid, ${ }^{1052}$ the total synthesis of $(\mathrm{R}, \mathrm{R}, \mathrm{R})-\alpha$-tocopherol, ${ }^{1053}$ the stereoselective total syntheses of lycopodium alkaloids, ${ }^{1054}$ the synthetic studies towards bridgehead diprenylsubstituted bicyclol[3.3.1]nonane-2,9-diones, ${ }^{1055}$ the total synthesis of (-)-pseudolaric acid B, 1056 the synthesis of azadirachtin, ${ }^{1057}$ the total synthesis of ( \pm )-phomactin B2, ${ }^{1058}$ the stereoselective total synthesis of arenastatin $\mathrm{A},{ }^{1059}$ the stereoselective formal total synthesis of (+)-hyperaspine, ${ }^{1060}$ the asymmetric synthesis of salvinorin $\mathrm{A}, 1061$ the asymmetric syntheses of heliannuols B and D, ${ }^{985}$ the total synthesis of C16 analogs of (-)-dictyostatin, 1062 the total synthesis of racemic clusianone and a formal synthesis of racemic garsubellin A, 1063 the synthesis of 2,6-disubstituted dihydropyranones, ${ }^{1064}$ the enantioselective synthesis of hydrobenzofuranones, ${ }^{1065}$ the synthesis of di- and trisaccharide mimetics with non-glycosidic amino bridges, ${ }^{1066}$ the total synthesis of $(4 R, 5 S)$-melithiazole $\mathrm{C}$ and $(3 R, 4 S)$-cystothiazole $\mathrm{E}$, 1067 the synthesis of trifluoromethylated cyclodextrin derivatives, ${ }^{1068}$ the asymmetric total syntheses of ecteinascidin 597 and ecteinascidin 583,1069 the enantioselective total synthesis of (-)-erinacine $\mathrm{B},{ }^{1070}$ the synthesis of the C31-C67 fragment of amphidinol 3, ${ }^{1071}$ the total synthesis of (-)-himgaline, ${ }^{1072}$ the total synthesis of pseudolaric acid A, ${ }^{1073}$ and the total synthesis of (-)-sarain A. ${ }^{1074}$

The unique oxidizing properties of DMP can be illustrated by its application in the total synthesis of the CP-molecules, lead structures for cardiovascular and anticancer drugs, published by Nicolaou and co-workers. ${ }^{1075-1077}$ In this synthetic investigation, a hindered secondary alcohol $\mathbf{5 7 5}$ was oxidized with DMP to the stable diol $\mathbf{5 7 7}$ through intermediate hemiketal 576 (Scheme 197).

The practical value of DMP as a reagent was recently extended to a variety of other synthetically useful oxidative transformations, such as: the synthesis of various polycyclic heterocycles via the oxidative cascade cyclization of anilides with pendant double bonds, ${ }^{1078}$ the oxidative aromatization of 1,4-dihydropyridines, ${ }^{1079}$ the one-pot oxidative allylation of Morita-BaylisHillman adducts with allyltrimethylsilane promoted by $\mathrm{DMP} / \mathrm{BF}_{3} \cdot \mathrm{OEt}_{2}, 1080$ the DMP promoted oxidative coupling of Baylis-Hillman adducts with silyl enol ethers, ${ }^{1081}$ the synthesis of 2-amino-1,4-benzoquinone-4-phenylimides from anilines via DMP oxidation, ${ }^{1082}$ the $\alpha$ bromination of 1,3-dicarbonyl compounds using DMP and tetraethylammonium bromide, 
1083 the decarboxylative bromination of $\alpha, \beta$-unsaturated carboxylic acids with DMP and tetraethylammonium bromide, ${ }^{1084}$ the $\alpha$-tosyloxylation of ketones using DMP and $p$ toluenesulfonic acid, ${ }^{1085}$ the solvent-free synthesis of 1-( $p$-toluenesulfonyloxy)-1,2benziodoxol-3(1H)-one from DMP and $p$-toluenesulfonic acid and its subsequent utilitization for $\alpha$-tosyloxylation of ketones, ${ }^{1086}$ the synthesis of 2 -substituted benzothiazoles $\mathbf{5 7 9}$ via oxidative cyclization of thioformanilides $\mathbf{5 7 8}$ (Scheme 198), ${ }^{381}$ the synthesis of thioesters $\mathbf{5 8 2}$ from the corresponding aldehydes $\mathbf{5 8 0}$ and thiols $\mathbf{5 8 1}$ under mild conditions (Scheme 199), 1087 and the synthesis of imides (e.g. 583), N-acyl vinylogous carbamates and ureas, and nitriles by the oxidation of amides and amines with DMP (Scheme 200). ${ }^{1088}$

\section{Conclusions}

The preceding survey of the recent developments in the chemistry of polyvalent iodine compounds reflects an active current interest in this highly versatile class of valuable reagents. From the practical point of view, especially important are the simplest, traditional reagents, such as (diacetoxyiodo)benzene and iodosylbenzene, which have been increasingly employed in organic synthesis. This growing interest in iodine(III) compounds is mainly due to their very useful oxidizing properties, combined with their benign environmental character and commercial availability.

There has been a major surge of activity in several areas of organic polyvalent iodine chemistry. These areas include the synthetic applications of IBX and similar oxidizing reagents based on the iodine $(\mathrm{V})$ derivatives, the development and synthetic use of polymer-supported and recyclable polyvalent iodine reagents, structural studies of complexes and supramolecular assemblies of polyvalent iodine compounds, the catalytic applications of organoiodine compounds, and the transition metal catalyzed reactions of various hypervalent iodine reagents.

We hope and anticipate that this review will provide additional stimulus for the further development of the chemistry of polyvalent iodine compounds.

\section{Acknowledgments}

Our own work described here was supported by the National Science Foundation (NSF/CHE-0702734) at Minnesota and by the National Institute of Health [GM-57052] at Utah.

\section{References}

1. Wirth, T., editor. Top Curr Chem. Vol. 224. 2003. Hypervalent Iodine Chemistry: Modern Developments in Organic Synthesis; p. 2003

2. Varvoglis, A. The Organic Chemistry of Polycoordinated Iodine. VCH Publishers, Inc.; New York: 1992.

3. Varvoglis, A. Hypervalent Iodine in Organic Synthesis. Academic Press; London: 1997.

4. Moriarty, RM.; Prakash, O. Hypervalent Iodine in Organic Chemistry: Chemical Transformations. Wiley-Interscience; 2008.

5. Stang PJ, Zhdankin VV. Chem Rev 1996;96:1123. [PubMed: 11848783]

6. Zhdankin VV, Stang PJ. Chem Rev 2002;102:2523. [PubMed: 12105935]

7. Wirth T, Hirt UH. Synthesis 1999:1271.

8. Stang PJ. J Org Chem 2003;68:2997. [PubMed: 12688766]

9. Moriarty RM. J Org Chem 2005;70:2893. [PubMed: 15822947]

10. Zhdankin VV. Science of Synthesis 2007;31a:161.

11. Wirth T. Angew Chem, Int Ed 2005;44:3656.

12. Matveeva ED, Proskurnina MV, Zefirov NS. Heteroatom Chem 2006;17:595.

13. Kitamura T, Fujiwara Y. Org Prep Proced Int 1997;29:409. 
14. Varvoglis A. Tetrahedron 1997;53:1179.

15. Zhdankin, VV.; Stang, PJ. Chemistry of Hypervalent Compounds. Akiba, Ky, editor. VCH Publishers; New York: 1999.

16. Koser, GF. Chemistry of Halides, Pseudo-Halides and Azides, Suppl D2. Patai, S.; Rappoport, Z., editors. Wiley-Interscience; Chichester: 1995.

17. Zhdankin VV. Speciality Chemicals Magazine 2002;22:38.

18. Deprez NR, Sanford MS. Inorg Chem 2007;46:1924. [PubMed: 17348723]

19. Ochiai M. Coord Chem Rev 2006;250:2771.

20. Richardson RD, Wirth T. Angew Chem, Int Ed 2006;45:4402.

21. Silva LF Jr. Molecules 2006;11:421. [PubMed: 17962775]

22. Ladziata U, Zhdankin VV. ARKIVOC 2006;ix:26.

23. Ladziata U, Zhdankin VV. Synlett 2007:527.

24. Zhdankin VV. Curr Org Synth 2005;2:121.

25. Koser GF. Adv Heterocycl Chem 2004;86:225.

26. Tohma H, Kita Y. Adv Synth Catal 2004;346:111.

27. Yoneda N. J Fluorine Chem 2004;125:7.

28. Dauban P, Dodd RH. Synlett 2003:1571.

29. Feldman KS. ARKIVOC 2003;vi:179.

30. Togo H, Sakuratani K. Synlett 2002:1966.

31. Moreno I, Tellitu I, Herrero MT, SanMartin R, Dominguez E. Curr Org Chem 2002;6:1433.

32. Moriarty RM, Prakash O. Org React 2001;57:327.

33. Morales-Rojas H, Moss RA. Chem Rev 2002;102:2497. [PubMed: 12105934]

34. Moore JD, Hanson PR. Chemtracts 2002;15:74.

35. Togo H, Katohgi M. Synlett 2001:565.

36. Ochiai M. J Organomet Chem 2000;611:494.

37. Moriarty RM, Prakash O. Org React 1999;54:273.

38. Ochiai, M. Chemistry of Hypervalent Compounds. Akiba, Ky, editor. VCH Publishers; New York: 1999.

39. Muraki T, Togo H, Yokoyama M. Rev Heteroatom Chem 1997;17:213.

40. Moriarty RM, Prakash O. Adv Heterocycl Chem 1998;69:1.

41. Koser GF. Aldrichimica Acta 2001;34:89.

42. Zhdankin VV, Stang PJ. Tetrahedron 1998;54:10927.

43. Grushin VV. Chem Soc Rev 2000;29:315.

44. Umemoto T. Chem Rev 1996;96:1757. [PubMed: 11848810]

45. Zhdankin VV. Rev Heteroat Chem 1997;17:133.

46. Pirkuliev NS, Brel VK, Zefirov NS. Russ Chem Rev 2000;69:105.

47. Okuyama T. Acc Chem Res 2002;35:12. [PubMed: 11790084]

48. French AN, Bissmire S, Wirth T. Chem Soc Rev 2004;33:354. [PubMed: 15280968]

49. Ochiai M. Chem Rec 2007;7:12. [PubMed: 17304588]

50. Quideau S, Pouysegu L, Deffieux D. Curr Org Chem 2004;8:113.

51. Ciufolini MA, Braun NA, Canesi S, Ousmer M, Chang J, Chai D. Synthesis 2007:3759.

52. Kita Y, Fujioka H. Pure Appl Chem 2007;79:701.

53. Rodriguez S, Wipf P. Synthesis 2004:2767.

54. Okuyama T, Fujita M. Russ J Org Chem 2005;41:1245.

55. Okuyama T, Fujita M. ACS Symp Ser 2007;965:68.

56. Kirmse W. Eur J Org Chem 2005:237.

57. Muller P, Allenbach YF, Chappellet S, Ghanem A. Synthesis 2006:1689.

58. Muller P. Acc Chem Res 2004;37:243. [PubMed: 15096061]

Chem Rev. Author manuscript; available in PMC 2009 December 10. 
59. Holsworth, DD. Name Reactions for Functional Group Transformations. Li, JJ.; Corey, EJ., editors. John Wiley \& Sons, Inc.; Hoboken, N. J.: 2007.

60. Quideau S, Pouysegu L, Deffieux D. Synlett 2008:467.

61. Frohn H-J, Hirschberg ME, Wenda A, Bardin VV. J Fluorine Chem 2008;129:459.

62. Akiba, Ky. Chemistry of Hypervalent Compounds. Akiba, Ky, editor. VCH Publishers; New York: 1999.

63. Landrum GA, Goldberg N, Hoffmann R. J Chem Soc, Dalton Trans 1997:3605.

64. Landrum GA, Goldberg N, Hoffmann R, Minyaev RM. New J Chem 1998;22:883.

65. Kiprof P, Zhdankin V. ARKIVOC 2003;vi:170.

66. Kiprof P. ARKIVOC 2005;iv:19.

67. Ochiai M, Sueda T, Miyamoto K, Kiprof P, Zhdankin VV. Angew Chem, Int Ed 2006;45:8203.

68. Okuyama T, Yamataka H. Can J Chem 1999;77:577.

69. Carroll MA, Martin-Santamaria S, Pike VW, Rzepa HS, Widdowson DA. J Chem Soc, Perkin Trans 2 1999:2707.

70. Martin-Santamaria S, Carroll MA, Carroll CM, Carter CD, Rzepa HS, Widdowson DA, Pike VW. Chem Commun 2000:649.

71. Martin-Santamaria S, Carroll MA, Pike VW, Rzepa HS, Widdowson DA. J Chem Soc, Perkin Trans 2 2000:2158

72. Su JT, Goddard WA III. J Am Chem Soc 2005;127:14146. [PubMed: 16218584]

73. Boucher M, Macikenas D, Ren T, Protasiewicz JD. J Am Chem Soc 1997;119:9366.

74. Zhdankin VV, Arbit RM, Lynch BJ, Kiprof P, Young VG. J Org Chem 1998;63:6590.

75. Hirt UH, Schuster MFH, French AN, Wiest OG, Wirth T. Eur J Org Chem 2001:1569.

76. Pouysegu L, Chassaing S, Dejugnac D, Lamidey A-M, Miqueu K, Sotiropoulos J-M, Quideau S. Angew Chem, Int Ed 2008;47:3552.

77. Bakalbassis EG, Spyroudis S, Tsiotra E. J Org Chem 2006;71:7060. [PubMed: 16930064]

78. Ochiai M, Miyamoto K, Shiro M, Ozawa T, Yamaguchi K. J Am Chem Soc 2003;125:13006. [PubMed: 14570456]

79. Ochiai M, Miyamoto K, Suefuji T, Sakamoto S, Yamaguchi K, Shiro M. Angew Chem, Int Ed 2003;42:2191.

80. Ochiai M, Miyamoto K, Suefuji T, Shiro M, Sakamoto S, Yamaguchi K. Tetrahedron 2003;59:10153.

81. Ochiai M, Miyamoto K, Yokota Y, Suefuji T, Shiro M. Angew Chem, Int Ed 2004;44:75.

82. Ochiai M, Suefuji T, Miyamoto K, Tada N, Goto S, Shiro M, Sakamoto S, Yamaguchi K. J Am Chem Soc 2003;125:769. [PubMed: 12526677]

83. Suefuji T, Shiro M, Yamaguchi K, Ochiai M. Heterocycles 2006;67:391.

84. Ochiai M, Suefuji T, Miyamoto K, Shiro M. Chem Commun 2003:1438.

85. Zhdankin VV, Koposov AY, Yashin NV. Tetrahedron Lett 2002;43:5735.

86. Zhdankin VV, Koposov AE, Smart JT, Tykwinski RR, McDonald R, Morales-Izquierdo A. J Am Chem Soc 2001;123:4095. [PubMed: 11457167]

87. Richter HW, Koser GF, Incarvito CD, Rheingold AL. Inorg Chem 2007;46:5555. [PubMed: 17569525]

88. Koposov AY, Netzel BC, Yusubov MS, Nemykin VN, Nazarenko AY, Zhdankin VV. Eur J Org Chem 2007:4475.

89. Macikenas D, Skrzypczak-Jankun E, Protasiewicz JD. Angew Chem, Int Ed 2000;39:2007.

90. Macikenas D, Skrzypczak-Jankun E, Protasiewicz JD. J Am Chem Soc 1999;121:7164.

91. Meprathu BV, Protasiewicz JD. ARKIVOC 2003;vi:83.

92. Meprathu BV, Justik MW, Protasiewicz JD. Tetrahedron Lett 2005;46:5187.

93. Koposov AY, Nemykin VN, Zhdankin VV. New J Chem 2005;29:998.

94. Zhdankin VV, Koposov AY, Netzel BC, Yashin NV, Rempel BP, Ferguson MJ, Tykwinski RR. Angew Chem, Int Ed 2003;42:2194.

95. Zhdankin VV, Litvinov DN, Koposov AY, Luu T, Ferguson MJ, McDonald R, Tykwinski RR. Chem Commun 2004:106. 
96. Zhdankin VV, Koposov AY, Litvinov DN, Ferguson MJ, McDonald R, Luu T, Tykwinski RR. J Org Chem 2005;70:6484. [PubMed: 16050713]

97. Ladziata U, Koposov AY, Lo KY, Willging J, Nemykin VN, Zhdankin VV. Angew Chem, Int Ed 2005;44:7127.

98. Koposov AY, Karimov RR, Geraskin IM, Nemykin VN, Zhdankin VV. J Org Chem 2006;71:8452. [PubMed: 17064019]

99. Nikiforov VA, Karavan VS, Miltsov SA, Selivanov SI, Kolehmainen E, Wegelius E, Nissine M. ARKIVOC 2003;vi:191.

100. Batchelor RJ, Birchall T, Sawyer JF. Inorg Chem 1986;25:1415.

101. Zhdankin VV, Maydanovych O, Herschbach J, McDonald R, Tykwinski RR. J Am Chem Soc 2002;124:11614. [PubMed: 12296724]

102. Zhdankin VV, Koposov AY, Su LS, Boyarskikh VV, Netzel BC, Young VG. Org Lett 2003;5:1583. [PubMed: 12713329]

103. Yusubov MS, Funk TV, Chi K-W, Cha E-H, Kim GH, Kirschning A, Zhdankin VV. J Org Chem 2008;73:295. [PubMed: 18062703]

104. Koposov AY, Litvinov DN, Zhdankin VV, Ferguson MJ, McDonald R, Tykwinski RR. Eur J Org Chem 2006:4791.

105. Dess DB, Wilson SR, Martin JC. J Am Chem Soc 1993;115:2488.

106. Dess DB, Martin JC. J Am Chem Soc 1991;113:7277.

107. Stevenson PJ, Treacy AB, Nieuwenhuyzen M. J Chem Soc, Perkin Trans 2 1997:589.

108. Hiller A, Patt JT, Steinbach J. Magn Reson Chem 2006;44:955. [PubMed: 16900572]

109. Cerioni G, Uccheddu G. Tetrahedron Lett 2004;45:505.

110. Mocci F, Uccheddu G, Frongia A, Cerioni G. J Org Chem 2007;72:4163. [PubMed: 17465567]

111. Richter HW, Cherry BR, Zook TD, Koser GF. J Am Chem Soc 1997;119:9614.

112. Silva LF, Lopes NP. Tetrahedron Lett 2005;46:6023.

113. Saltzman H, Sharefkin JG. Org Synth Coll Vol V 1973:658.

114. Gao Y, Jiao X, Fan W, Shen J-K, Shi Q, Basolo F. J Coord Chem 1993;29:349.

115. Lucas HJ, Kennedy ER, Formo MW. Org Synth Coll Vol III 1955:483.

116. Sawaguchi M, Ayuba S, Hara S. Synthesis 2002:1802.

117. Schardt BC, Hill CL. Inorg Chem 1983;22:1563.

118. McQuaid KM, Pettus TRR. Synlett 2004:2403. [PubMed: 19079634]

119. Carmalt CJ, Crossley JG, Knight JG, Lightfoot P, Martin A, Muldowney MP, Norman NC, Orpen AG. J Chem Soc, Chem Commun 1994:2367.

120. Barea G, Maseras F, Lledos A. New J Chem 2003;27:811.

121. Koposov AY, Netzel BC, Yusubov Mekhman S, Nemykin VN, Nazarenko AY, Zhdankin VV. Eur J Org Chem 2007:4475.

122. Ochiai M, Miyamoto K, Yokota Y, Suefuji T, Shiro M. Angew Chem, Int Ed 2005;44:75.

123. Tohma H, Takizawa S, Maegawa T, Kita Y. Angew Chem, Int Ed 2000;39:1306.

124. Tohma H, Maegawa T, Takizawa S, Kita Y. Adv Synth Catal 2002;344:328.

125. Tohma H, Takizawa S, Watanabe H, Kita Y. Tetrahedron Lett 1998;39:4547.

126. Moriarty RM, Duncan MP, Prakash O. J Chem Soc, Perkin Trans 1 1987:1781.

127. Moriarty RM, Rani N, Condeiu C, Duncan MP, Prakash O. Synth Commun 1997;27:3273.

128. Francisco CG, Herrera AJ, Suarez E. J Org Chem 2002;67:7439. [PubMed: 12375977]

129. Francisco CG, Freire R, Gonzalez CC, Leon EI, Riesco-Fagundo C, Suarez E. J Org Chem 2001;66:1861. [PubMed: 11262138]

130. Francisco CG, Herrera AJ, Suarez E. J Org Chem 2003;68:1012. [PubMed: 12558429]

131. Huang W-J, Singh OV, Chen C-H, Chiou S-Y, Lee S-S. Helv Chim Acta 2002;85:1069.

132. Tada N, Miyamoto K, Ochiai M. Chem Pharm Bull 2004;52:1143. [PubMed: 15340208]

133. Ueno M, Nabana T, Togo H. J Org Chem 2003;68:6424. [PubMed: 12895080]

134. Fujita M, Lee HJ, Sugimura T, Okuyama T. Chem Commun 2007:1139. 
135. Miyamoto K, Tada N, Ochiai M. J Am Chem Soc 2007;129:2772. [PubMed: 17309268]

136. Miyamoto K, Hirobe M, Saito M, Shiro M, Ochiai M. Org Lett 2007;9:1995. [PubMed: 17417863]

137. Ono T, Henderson P. Tetrahedron Lett 2002;43:7961.

138. Lee S, MacMillan DWC. Tetrahedron 2006;62:11413.

139. Yusubov MS, Gilmkhanova MP, Zhdankin VV, Kirschning A. Synlett 2007:563.

140. Kirschning A, Yusubov MS, Yusubova RY, Chi K-W, Park JY. Beilstein J Org Chem 2007;3:19. [PubMed: 17543133]

141. Simonneaux G, Tagliatesta P. J Porphyrins Phthalocyanines 2004;8:1166.

142. Groves JT. J Porphyrins Phthalocyanines 2000;4:350.

143. Rose E, Andrioletti B, Zrig S, Quelquejeu-Etheve M. Chem Soc Rev 2005;34:573. [PubMed: 15965539]

144. Bernadou J, Meunier B. Adv Synth Catal 2004;346:171.

145. Vinhado FS, Martins PR, Iamamoto Y. Curr Top Catal 2002;3:199.

146. Kang M-J, Song WJ, Han A-R, Choi YS, Jang HG, Nam W. J Org Chem 2007;72:6301. [PubMed: 17622172]

147. de Visser SP, Oh K, Han A-R, Nam W. Inorg Chem 2007;46:4632. [PubMed: 17444641]

148. Silva, GdF; Carvalho da Silva, D.; Guimaraes, AS.; do Nascimento, E.; Reboucas, JS.; Peres de Araujo, M.; Dai de Carvalho, MEM.; Idemori, YM. J Mol Cat A 2007;266:274.

149. Song WJ, Seo MS, DeBeer George S, Ohta T, Song R, Kang M-J, Tosha T, Kitagawa T, Solomon EI, Nam W. J Am Chem Soc 2007;129:1268. [PubMed: 17263410]

150. Lindsay Smith JR, Iamamoto Y, Vinhado FS. J Mol Cat A 2006;252:23.

151. Miyachi H, Nagatsu Y. Chem Pharm Bull 2002;50:1137. [PubMed: 12192156]

152. Murakami Y, Konishi K. J Am Chem Soc 2007;129:14401. [PubMed: 17967018]

153. Santos MMC, Silva AMS, Cavaleiro JAS, Levai A, Ptonay T. Eur J Org Chem 2007:2877.

154. Babakhania R, Bahadoran F, Safari N. J Porphyrins Phthalocyanines 2007;11:95.

155. Pouralimardan O, Chamayou A-C, Janiak C, Hosseini-Monfared H. Inorg Chim Acta 2007;360:1599.

156. Santos CMM, Silva AMS, Cavaleiro JAS, Patonay T, Levai A. J Heterocycl Chem 2006;43:1319.

157. Collman JP, Zeng L, Wang HJH, Lei A, Brauman JI. Eur J Org Chem 2006:2707.

158. Suh Y, Seo MS, Kim KM, Kim YS, Jang HG, Tosha T, Kitagawa T, Kim J, Nam W. J Inorg Biochem 2006;100:627. [PubMed: 16458358]

159. Das P, Kuzniarska-Biernacka I, Silva AR, Carvalho AP, Pires J, Freire C. J Mol Cat A 2006;248:135.

160. Park S-E, Song WJ, Ryu YO, Lim MH, Song R, Kim KM, Nam W. J Inorg Biochem 2005;99:424. [PubMed: 15621274]

161. Collman JP, Zeng L, Brauman JI. Inorg Chem 2004;43:2672. [PubMed: 15074985]

162. Rosa Silva A, Freire C, de Castro B. New J Chem 2004;28:253.

163. Wang SH, Mandimutsira BS, Todd R, Ramdhanie B, Fox JP, Goldberg DP. J Am Chem Soc 2004;126:18. [PubMed: 14709038]

164. Silva AR, Vital J, Figueiredo JL, Freire C, de Castro B. New J Chem 2003;27:1511.

165. Zolezzi S, Spodine E, Decinti A. Polyhedron 2003;22:1653.

166. Davoras EM, Coutsolelos AG. J Inorg Biochem 2003;94:161. [PubMed: 12620687]

167. Poriel C, Ferrand Y, Le Maux P, Rault-Berthelot J, Simonneaux G. Tetrahedron Lett 2003;44:1759.

168. Liu H-Y, Lai T-S, Yeung L-L, Chang CK. Org Lett 2003;5:617. [PubMed: 12605473]

169. Jitsukawa K, Shiozaki H, Masuda H. Tetrahedron Lett 2002;43:1491.

170. Yang ZW, Kang QX, Quan F, Lei ZQ. J Mol Cat A 2007;261:190.

171. Vatele J-M. Synlett 2006:2055.

172. Murahashi S-I, Noji S, Hirabayashi T, Komiya N. Synlett 2004:1739.

173. Wolckenhauer SA, Devlin AS, Du Bois J. Org Lett 2007;9:4363. [PubMed: 17887696]

174. Ferrand Y, Daviaud R, Le Maux P, Simonneaux G. Tetrahedron: Asymmetry 2006;17:952.

175. Bryliakov KP, Talsi EP. Chem Eur J 2007;13:8045. 
176. Zupan M, Pollak A. J Fluorine Chem 1976;7:445.

177. Gregorcic A, Zupan M. Bull Chem Soc Jpn 1977;50:517.

178. Abo-Amer A, Frohn H-J, Steinberg C, Westphal U. J Fluorine Chem 2006;127:1311.

179. Sheremetev AB, Dmitriev DE, Konkina SM. Russ Chem Bull 2004;53:1130.

180. Bailly E, Barthen P, Breuer W, Frohn HJ, Giesen M, Helber J, Henkel G, Priwitzer A. Z Anorg Allg Chem 2000;626:1406.

181. Padelidakis V, Tyrra W, Naumann D. J Fluorine Chem 1999;99:9.

182. Frohn HJ, Bardin VV. J Fluorine Chem 2005; 126:1036.

183. Ye C, Twamley B, Shreeve JM. Org Lett 2005;7:3961. [PubMed: 16119942]

184. Kasumov TM, Pirguliyev NS, Brel VK, Grishin YK, Zefirov NS, Stang PJ. Tetrahedron 1997;53:13139.

185. Pirkuliyev NS, Brel’ VK, Zhdankin VV, Zefirov NS. Russ J Org Chem 2002;38:1224.

186. Fuchigami T, Fujita T. J Org Chem 1994;59:7190.

187. Hara S, Hatakeyama T, Chen S-Q, Ishi-i K, Yoshida M, Sawaguchi M, Fukuhara T, Yoneda N. J Fluorine Chem 1998;87:189.

188. Carpenter WR. J Org Chem 1966;31:2688.

189. Arrica MA, Wirth T. Eur J Org Chem 2005:395.

190. Minkwitz R, Berkei M. Inorg Chem 1998;37:5247.

191. Choo J, Kim S, Joo H, Kwon Y. THEOCHEM 2002;587:1.

192. Porter, CW. PhD Thesis. The University of Akron; 1996. Structural analyses using x-ray crystallography ((difluoroiodo)toluene, hydroxymethylbenziodoxathiole).

193. Hara S, Sekiguchi M, Ohmori A, Fukuhara T, Yoneda N. J Chem Soc, Chem Commun 1996:1899. 194. Yoshida M, Fujikawa K, Sato S, Hara S. ARKIVOC 2003;vi:36.

195. Sato S, Yoshida M, Hara S. Synthesis 2005:2602.

196. Motherwell WB, Greaney MF, Tocher DA. J Chem Soc, Perkin Trans 1 2002:2809.

197. Motherwell WB, Greaney MF, Edmunds JJ, Steed JW. J Chem Soc, Perkin Trans 1 2002:2816.

198. Inagaki T, Nakamura Y, Sawaguchi M, Yoneda N, Ayuba S, Hara S. Tetrahedron Lett 2003;44:4117.

199. Furrow ME, Myers AG. J Am Chem Soc 2004;126:12222. [PubMed: 15453728]

200. Hara S, Nakahigashi J, Ishi-i K, Sawaguchi M, Sakai H, Fukuhara T, Yoneda N. Synlett 1998:495.

201. Hara S, Nagahigashi J, Ishi-i K, Fukuhara T, Yoneda N. Tetrahedron Lett 1998;39:2589.

202. Conte P, Panunzi B, Tingoli M. Tetrahedron Lett 2005;47:273.

203. Panunzi B, Picardi A, Tingoli M. Synlett 2004:2339.

204. Ochiai M, Hirobe M, Yoshimura A, Nishi Y, Miyamoto K, Shiro M. Org Lett 2007;9:3335.

[PubMed: 17658841]

205. Yoshida M, Osafune K, Hara S. Synthesis 2007:1542.

206. Yoshida M, Kawakami K, Hara S. Synthesis 2004:2821.

207. Frohn H-J, Bardin VV. Z Anorg Allg Chem 2008;634:82.

208. Frohn H-J, Wenda A, Floerke U. Z Anorg Allg Chem 2008;634:764.

209. Lucas HJ, Kennedy ER. Org Synth Coll Vol III 1955:482.

210. Zanka A, Takeuchi H, Kubota A. Org Process Res Dev 1998;2:270.

211. Yusubov MS, Drygunova LA, Zhdankin VV. Synthesis 2004:2289.

212. Obeid N, Skulski L. Molecules 2001;6:869.

213. Baranowski A, Plachta D, Skulski L, Klimaszewska M. J Chem Res, Synop 2000:435.

214. Zielinska A, Skulski L. Tetrahedron Lett 2004;45:1087.

215. Zhao X-F, Zhang C. Synthesis 2007:551.

216. Lulinski P, Obeid N, Skulski L. Bull Chem Soc Jpn 2001;74:2433.

217. Zefirov NS, Safronov SO, Kaznacheev AA, Zhdankin VV. Zh Org Khim 1989;25:1807.

218. Salamant W, Hulme C. Tetrahedron Lett 2006;47:605.

219. Archer EM, van Schalkwyk TGD. Acta Cryst 1953;6:88.

Chem Rev. Author manuscript; available in PMC 2009 December 10. 
220. Bekoe DA, Hulme R. Nature 1956;177:1230.

221. Carey JV, Chaloner PA, Hitchcock PB, Neugebauer T, Seddon KR. J Chem Res, Synop 1996:358. 222. Mishra AK, Olmstead MM, Ellison JJ, Power PP. Inorg Chem 1995;34:3210.

223. Protasiewicz JD. J Chem Soc, Chem Commun 1995:1115.

224. Grebe J, Geiseler G, Harms K, Dehnicke K. Z Naturforsch, B: Chem Sci 1999;54:140.

225. Montanari V, DesMarteau DD, Pennington WT. J Mol Str 2000;550-551:337.

226. Masson S, Thuillier A. Bull Soc Chim Fr 1969:4368.

227. Yusubov MS, Yusubova RJ, Filimonov VD, Chi K-W. Synth Commun 2004;34:443.

228. Yusubov MS, Drygunova LA, Tkachev AV, Zhdankin VV. ARKIVOC 2005;iv:179.

229. Ibrahim H, Kleinbeck F, Togni A. Helv Chim Acta 2004;87:605.

230. Jin L-M, Yin J-J, Chen L, Guo C-C, Chen Q-Y. Synlett 2005:2893.

231. Telvekar VN. Synth Commun 2007;37:2647.

232. Benjahad A, Guillemont J, Andries K, Nguyen CH, Grierson DS. Bioorg Med Chem Lett 2003;13:4309. [PubMed: 14643315]

233. Benjahad A, Oumouch S, Guillemont J, Pasquier E, Mabire D, Andries K, Nguyen CH, Grierson DS. Bioorg Med Chem Lett 2007;17:712. [PubMed: 17157017]

234. Prakash O, Kaur H, Batra H, Rani N, Singh SP, Moriarty RM. J Org Chem 2001;66:2019. [PubMed: 11300895]

235. Prakash O, Sharma V, Batra H, Moriarty RM. Tetrahedron Lett 2001;42:553.

236. Kita Y, Takeda Y, Okuno T, Egi M, Iio K, Kawaguchi K-I, Akai S. Chem Pharm Bull 1997;45:1887.

237. Prakash O, Kaur H, Pundeer R, Dhillon RS, Singh SP. Synth Commun 2003;33:4037.

238. Li X-Q, Zhao X-F, Zhang C. Synthesis 2008:2589.

239. Cook TR, Esswein AJ, Nocera DG. J Am Chem Soc 2007;129:10094. [PubMed: 17655239]

240. Cotton FA, Koshevoy IO, Lahuerta P, Murillo CA, Sanau M, Ubeda MA, Zhao Q. J Am Chem Soc 2006;128:13674. [PubMed: 17044680]

241. Whitfield SR, Sanford MS. J Am Chem Soc 2007;129:15142. [PubMed: 18004863]

242. Khusniyarov MM, Harms K, Sundermeyer J. J Fluorine Chem 2006;127:200.

243. Hayton TW, Legzdins P, Patrick BO. Inorg Chem 2002;41:5388. [PubMed: 12377032]

244. Bastian M, Morales D, Poli R, Richard P, Sitzmann H. J Organomet Chem 2002;654:109.

245. Kalyani D, Sanford MS. J Am Chem Soc 2008;130:2150. [PubMed: 18229926]

246. Kalyani D, Dick AR, Anani WQ, Sanford MS. Tetrahedron 2006;62:11483.

247. Sharefkin JG, Saltzman H. Org Synth Coll Vol V 1973:660.

248. Ficht S, Mulbaier M, Giannis A. Tetrahedron 2001;57:4863.

249. Chen F-E, Xie B, Zhang P, Zhao J-F, Wang H, Zhao L. Synlett 2007:619.

250. Shang Y, But TYS, Togo H, Toy PH. Synlett 2007:67.

251. Qian W, Jin E, Bao W, Zhang Y. Angew Chem, Int Ed 2005;44:952.

252. Handy ST, Okello M. J Org Chem 2005;70:2874. [PubMed: 15787592]

253. Zhdankin VV, Scheuller MC, Stang PJ. Tetrahedron Lett 1993;34:6853.

254. Stang PJ, Zhdankin VV. J Am Chem Soc 1993;115:9808.

255. Gallop PM, Paz MA, Fluckiger R, Stang PJ, Zhdankin VV, Tykwinski RR. J Am Chem Soc 1993;115:11702.

256. Kazmierczak P, Skulski L, Kraszkiewicz L. Molecules 2001;6:881.

257. Lee BC, Lee KC, Lee H, Mach RH, Katzenellenbogen JA. Bioconjugate Chem 2007;18:514.

258. Ross TL, Ermert J, Hocke C, Coenen HH. J Am Chem Soc 2007;129:8018. [PubMed: 17536798]

259. Zielinska A, Skulski L. Molecules 2002;7:806.

260. Tohma H, Maruyama A, Maeda A, Maegawa T, Dohi T, Shiro M, Morita T, Kita Y. Angew Chem, Int Ed 2004;43:3595.

261. Dohi T, Maruyama A, Yoshimura M, Morimoto K, Tohma H, Shiro M, Kita Y. Chem Commun 2005:2205. 
262. Dohi T, Morimoto K, Takenaga N, Maruyama A, Kita Y. Chem Pharm Bull 2006;54:1608. [PubMed: 17077564]

263. Dohi T, Morimoto K, Takenaga N, Goto A, Maruyama A, Kiyono Y, Tohma H, Kita Y. J Org Chem 2007;72:109. [PubMed: 17194088]

264. Moroda A, Togo H. Tetrahedron 2006;62:12408.

265. Hossain MD, Kitamura T. Bull Chem Soc Jpn 2006;79:142.

266. Hossain D, Kitamura T. Synthesis 2005:1932.

267. Page TK, Wirth T. Synthesis 2006:3153.

268. McKillop A, Kemp D. Tetrahedron 1989;45:3299.

269. Ochiai M, Takaoka Y, Masaki Y, Nagao Y, Shiro M. J Am Chem Soc 1990;112:5677.

270. Ochiai M, Oshima K, Ito T, Masaki Y, Shiro M. Tetrahedron Lett 1991;32:1327.

271. Togo H, Nabana T, Yamaguchi K. J Org Chem 2000;65:8391. [PubMed: 11101405]

272. Daub KS, Habermann B, Hahn T, Teich L, Eger K. Eur J Org Chem 2004:894.

273. Rocaboy C, Gladysz JA. Chem Eur J 2003;9:88.

274. Fujita M, Okuno S, Lee HJ, Sugimura T, Okuyama T. Tetrahedron Lett 2007;48:8691.

275. Hossain MD, Kitamura T. J Org Chem 2005;70:6984. [PubMed: 16095332]

276. Hossain MD, Kitamura T. Tetrahedron Lett 2006;47:7889.

277. Stang PJ, Boehshar M, Wingert H, Kitamura T. J Am Chem Soc 1988;110:3272.

278. Sutherland A, Vederas JC. Chem Commun 2002:224.

279. Ray DG III, Koser GF. J Org Chem 1992;57:1607.

280. Koposov AY, Boyarskikh VV, Zhdankin VV. Org Lett 2004;6:3613. [PubMed: 15387561]

281. Merkushev EB, Novikov AN, Makarchenko SS, Moskal'chuk AS, Glushkova VV, Kogai TI,

Polyakova LG. J Org Chem USSR (Engl Trans) 1975;11:1246.

282. Das JP, Roy UK, Roy S. Organometallics 2005;24:6136.

283. Sheremetev AB, Konkina SM. Mendeleev Commun 2003:277.

284. Spyroudis S, Varvoglis A. Synthesis 1975:445.

285. Togo H, Aoki M, Yokoyama M. Tetrahedron 1993;49:8241.

286. Alcock NW, Harrison WD, Howes C. J Chem Soc, Dalton Trans 1984:1709.

287. De Mico A, Margarita R, Parlanti L, Vescovi A, Piancatelli G. J Org Chem 1997;62:6974.

288. Piancatelli G, Leonelli F, Do N, Ragan J. Org Synth 2006;83:18.

289. Pozzi G, Quici S, Shepperson I. Tetrahedron Lett 2002;43:6141.

290. Vatele J-M. Tetrahedron Lett 2006;47:715.

291. Vugts DJ, Veum L, al-Mafraji K, Lemmens R, Schmitz RF, de Kanter FJJ, Groen MB, Hanefeld U, Orru RVA. Eur J Org Chem 2006:1672.

292. Herrerias CI, Zhang TY, Li C-J. Tetrahedron Lett 2006;47:13.

293. But TYS, Tashino Y, Togo H, Toy PH. Org Biomol Chem 2005;3:970. [PubMed: 15750636]

294. Pozzi G, Cavazzini M, Quici S, Benaglia M, Dell'Anna G. Org Lett 2004;6:441. [PubMed: 14748613]

295. Holczknecht O, Cavazzini M, Quici S, Shepperson I, Pozzi G. Adv Synth Catal 2005;347:677.

296. Pozzi G, Cavazzini M, Holczknecht O, Quici S, Shepperson I. Tetrahedron Lett 2004;45:4249.

297. Qian W, Jin E, Bao W, Zhang Y. Tetrahedron 2006;62:556.

298. Hansen TM, Florence GJ, Lugo-Mas P, Chen J, Abrams JN, Forsyth CJ. Tetrahedron Lett 2002;44:57.

299. Li Y, Hale KJ. Org Lett 2007;9:1267. [PubMed: 17326647]

300. Tohma H, Maegawa T, Kita Y. Synlett 2003:723.

301. Karade NN, Shirodkar SG, Dhoot BM, Waghmare PB. J Chem Res 2005:274.

302. Karade NN, Tiwari GB, Huple DB. Synlett 2005:2039.

303. Karade NN, Budhewar VH, Katkar AN, Tiwari GB. ARKIVOC 2006;xi:162.

304. Kansara A, Sharma PK, Banerji KK. J Chem Res 2004:581.

305. Huang S, Wang F, Gan L, Yuan G, Zhou J, Zhang S. Org Lett 2006;8:277. [PubMed: 16408894]

Chem Rev. Author manuscript; available in PMC 2009 December 10. 
306. Moriarty RM, Hu H. Tetrahedron Lett 1981;22:2747.

307. Moriarty RM, Prakash O, Karalis P, Prakash I. Tetrahedron Lett 1984;25:4745.

308. Moriarty RM, Prakash O. J Org Chem 1985;50:151.

309. Moriarty RM, Prakash O, Thachet CT, Musallam HA. Heterocycles 1985;23:633.

310. Prakash O, Saini N, Tanwar MP, Moriarty RM. Contemp Org Synth 1995;2:121.

311. Ochiai M, Takeuchi Y, Katayama T, Sueda T, Miyamoto K. J Am Chem Soc 2005;127:12244.

[PubMed: 16131201]

312. Rao W, Chan PWH. Tetrahedron Lett 2007;48:3789.

313. Celik M, Alp C, Coskun B, Gueltekin MS, Balci M. Tetrahedron Lett 2006;47:3659.

314. Yusubov MS, Zholobova GA, Filimonova IL, Chi K-W. Russ Chem Bull 2004;53:1735.

315. Alvarez HM, Barbosa DP, Fricks AT, Aranda DAG, Valdes RH, Antunes OAC. Org Process Res Dev 2006;10:941.

316. Yu L, Chen B, Huang X. Tetrahedron Lett 2007;48:925.

317. Mironov YV, Sherman AA, Nifantiev NE. Tetrahedron Lett 2004;45:9107.

318. Shi M, Wang B-Y, Li J. Eur J Org Chem 2005:759.

319. Tellitu I, Dominguez E. Tetrahedron 2008;64:2465.

320. Tingoli M, Tiecco M, Chianelli D, Balducci R, Temperini A. J Org Chem 1991;56:6809.

321. Tingoli M, Tiecco M, Testaferri L, Balducci R. Synlett 1993:211.

322. Tingoli M, Tiecco M, Testaferri L, Temperini A. J Chem Soc, Chem Commun 1994:1883.

323. Tingoli M, Tiecco M, Testaferri L, Temperini A. Synth Commun 1998;28:1769.

324. Kirschning A, Plumeier C, Rose L. J Chem Soc, Chem Commun 1998:33.

325. Hashem A, Jung A, Ries M, Kirschning A. Synlett 1998:195.

326. Kirschning A, Kunst E, Ries M, Rose L, Schoenberger A, Wartchow R. ARKIVOC 2003;vi:145.

327. Sourkouni-Argirusi G, Kirschning A. Org Lett 2000;2:3781. [PubMed: 11101418]

328. Kirschning A, Monenschein H, Wittenberg R. Angew Chem, Int Ed 2001;40:650.

329. Merkushev EB. Synthesis 1988:923.

330. Panunzi B, Rotiroti L, Tingoli M. Tetrahedron Lett 2003;44:8753.

331. Yan J, Li J, Cheng D. Synlett 2007:2442.

332. Karade NN, Tiwari GB, Huple DB, Siddiqui TAJ. J Chem Res 2006:366.

333. Yusubov MS, Yusubova RY, Kirschning A, Park JY, Chi K-W. Tetrahedron Lett 2008;49:1506.

334. Cheng D-P, Chen Z-C, Zheng Q-G. Synth Commun 2003;33:2671.

335. Karade NN, Tiwari GB, Shirodkar SG, Dhoot BM. Synth Commun 2005;35:1197.

336. Catir M, Kilic H. Synlett 2004:2151.

337. Itoh N, Sakamoto T, Miyazawa E, Kikugawa Y. J Org Chem 2002;67:7424. [PubMed: 12375974]

338. Prakash O, Pundeer R, Kaur H. Synthesis 2003:2768.

339. Prakash O, Kaur H, Sharma V, Bhardwaj V, Pundeer R. Tetrahedron Lett 2004;45:9065.

340. Tellitu I, Urrejola A, Serna S, Moreno I, Herrero MT, Dominguez E, SanMartin R, Correa A. Eur J Org Chem 2007:437.

341. Herrero MT, Tellitu I, Dominguez E, Hernandez S, Moreno I, SanMartin R. Tetrahedron 2002;58:8581.

342. Herrero MT, Tellitu I, Dominguez E, Moreno I, SanMartin R. Tetrahedron Lett 2002;43:8273.

343. Correa A, Tellitu I, Dominguez E, Moreno I, SanMartin R. J Org Chem 2005;70:2256. [PubMed: 15760213]

344. Churruca F, SanMartin R, Tellitu I, Dominguez E. Eur J Org Chem 2005:2481.

345. Serna S, Tellitu I, Dominguez E, Moreno I, SanMartin R. Tetrahedron 2004;60:6533.

346. Churruca F, SanMartin R, Carril M, Urtiaga MK, Solans X, Tellitu I, Dominguez E. J Org Chem 2005;70:3178. [PubMed: 15822980]

347. Herrero MT, Tellitu I, Hernandez S, Dominguez E, Moreno I, SanMartin R. ARKIVOC 2002; v:31.

348. Serna S, Tellitu I, Dominguez E, Moreno I, SanMartin R. Tetrahedron Lett 2003;44:3483.

349. Correa A, Tellitu I, Dominguez E, SanMartin R. J Org Chem 2006;71:8316. [PubMed: 17025336]

Chem Rev. Author manuscript; available in PMC 2009 December 10. 
350. Serna S, Tellitu I, Dominguez E, Moreno I, SanMartin R. Org Lett 2005;7:3073. [PubMed: 15987208]

351. Tellitu I, Serna S, Herrero MT, Moreno I, Dominguez E, SanMartin R. J Org Chem 2007;72:1526. [PubMed: 17288399]

352. Correa A, Tellitu I, Dominguez E, SanMartin R. J Org Chem 2006;71:3501. [PubMed: 16626131]

353. Correa A, Tellitu I, Dominguez E, SanMartin R. Tetrahedron 2006;62:11100.

354. Huang J, Liang Y, Pan W, Yang Y, Dong D. Org Lett 2007;9:5345. [PubMed: 18047359]

355. Fan R, Wen F, Qin L, Pu D, Wang B. Tetrahedron Lett 2007;48:7444.

356. Huang X, Shao N, Palani A, Aslanian R. Tetrahedron Lett 2007;48:1967.

357. Mamaeva EA, Bakibaev AA. Tetrahedron 2003;59:7521.

358. Cheng D-P, Chen Z-C. Synth Commun 2002;32:2155.

359. Wardrop DJ, Zhang W, Landrie CL. Tetrahedron Lett 2004;45:4229.

360. Wardrop DJ, Burge MS. Chem Commun 2004:1230.

361. Wardrop DJ, Landrie CL, Ortiz JA. Synlett 2003:1352.

362. Wardrop DJ, Burge MS, Zhang W, Ortiz JA. Tetrahedron Lett 2003;44:2587.

363. Wardrop DJ, Zhang W. Org Lett 2001;3:2353. [PubMed: 11463314]

364. Wardrop DJ, Basak A. Org Lett 2001;3:1053. [PubMed: 11277793]

365. Wardrop DJ, Burge MS. J Org Chem 2005;70:10271. [PubMed: 16323835]

366. Kikugawa Y, Nagashima A, Sakamoto T, Miyazawa E, Shiiya M. J Org Chem 2003;68:6739. [PubMed: 12919042]

367. Mogilaiah K, Babu HR, Reddy NV. Synth Commun 2002;32:2377.

368. Prakash O, Bhardwaj V, Kumar R, Tyagi P, Aneja KR. Eur J Med Chem 2004;39:1073. [PubMed: 15571869]

369. Prakash O, Kumar R, Sharma D, Naithani R, Kumar R. Heteroatom Chem 2006;17:653.

370. Prakash O, Kumar R, Kumar R, Tyagi P, Kuhad RC. Eur J Med Chem 2007;42:868. [PubMed: 17222483]

371. Liu, S-j; Zhang, J-z; Tian, G-r; Liu, P. Synth Commun 2005;35:1753.

372. Zhang X, Gan L, Huang S, Shi Y. J Org Chem 2004;69:5800. [PubMed: 15307764]

373. Rao VS, Sekhar KC. Synth Commun 2004;34:2153.

374. Shang Z. Synth Commun 2006;36:2927.

375. Somogyi L. J Heterocycl Chem 2007;44:1235.

376. Kumar D, Chandra Sekhar KVG, Dhillon H, Rao VS, Varma RS. Green Chem 2004;6:156.

377. Aggarwal R, Sumran G. Synth Commun 2006;36:1873.

378. Huang H-Y, Hou R-S, Wang H-M, Chen L-C. Heterocycles 2005;65:1881.

379. Das B, Holla H, Mahender G, Venkateswarlu K, Bandgar BP. Synthesis 2005:1572.

380. Du Y, Liu R, Linn G, Zhao K. Org Lett 2006;8:5919. [PubMed: 17165894]

381. Bose DS, Idrees M. J Org Chem 2006;71:8261. [PubMed: 17025321]

382. Aggarwal R, Sumran G, Saini A, Singh SP. Tetrahedron Lett 2006;47:4969.

383. Mogilaiah K, Rani JU, Sakram B, Reddy NV. J Heterocycl Chem 2006;43:485.

384. Shang Z, Reiner J, Chang J, Zhao K. Tetrahedron Lett 2005;46:2701.

385. Karade NN, Tiwari GB, Gampawar SV. Synlett 2007:1921.

386. Prakash O, Pannu K. ARKIVOC 2007;xiii:28.

387. Du L-H, Wang Y-G. Synthesis 2007:675.

388. Das B, Srinivas Y, Holla H, Krishnaiah M, Narender R. Chem Lett 2007;36:1270.

389. Liu L-P, Lu J-M, Shi M. Org Lett 2007;9:1303. [PubMed: 17348667]

390. Huang X, Shao N, Palani A, Aslanian R, Buevich A, Seidel-Dugan C, Huryk R. Tetrahedron Lett 2008;49:3592.

391. Moroda A, Togo H. Synthesis 2008:1257.

392. Karade NN, Shirodkar SG, Patil MN, Potrekar RA, Karade HN. Tetrahedron Lett 2003;44:6729.

393. Boye AC, Meyer D, Ingison CK, French AN, Wirth T. Org Lett 2003;5:2157. [PubMed: 12790553] 
394. Biland AS, Altermann S, Wirth T. ARKIVOC 2003;vi:164.

395. Fujioka H, Matsuda S, Horai M, Fujii E, Morishita M, Nishiguchi N, Hata K, Kita Y. Chem Eur J 2007;13:5238.

396. Kita Y, Matsuda S, Fujii E, Horai M, Hata K, Fujioka H. Angew Chem, Int Ed 2005;44:5857.

397. Kita Y, Matsuda S, Fujii E, Kitagaki S, Inoguchi R, Hata K, Fujioka H. Heterocycles 2005;66:309.

398. Chanu A, Safir I, Basak R, Chiaroni A, Arseniyadis S. Eur J Org Chem 2007:4305.

399. Iglesias-Arteaga MA, Velazquez-Huerta GA. Tetrahedron Lett 2005;46:6897.

400. Iglesias-Arteaga MA, Arcos-Ramos RO. Tetrahedron Lett 2006;47:8029.

401. Iglesias-Arteaga MA, Arcos-Ramos RO, Mendez-Stivalet JM. Tetrahedron Lett 2007;48:7485.

402. Liu H, Tan C-H. Tetrahedron Lett 2007;48:8220.

403. Harayama Y, Yoshida M, Kamimura D, Kita Y. Chem Commun 2005:1764.

404. Harayama Y, Yoshida M, Kamimura D, Wada Y, Kita Y. Chem Eur J 2006;12:4893.

405. Li X, Xu Z, DiMauro EF, Kozlowski MC. Tetrahedron Lett 2002;43:3747.

406. Abo T, Sawaguchi M, Senboku H, Hara S. Molecules 2005;10:183. [PubMed: 18007285]

407. Zhang, L-h; Kauffman, GS.; Pesti, JA.; Yin, J. J Org Chem 1997;62:6918.

408. Zhang, L-h; Chung, JC.; Costello, TD.; Valvis, I.; Ma, P.; Kauffman, S.; Ward, R. J Org Chem 1997;62:2466. [PubMed: 11671583]

409. Prakash O, Batra H, Kaur H, Sharma PK, Sharma V, Singh SP, Moriarty RM. Synthesis 2001:541.

410. Song H, Chen W, Wang Y, Qin Y. Synth Commun 2005;35:2735.

411. Davis MC, Stasko D, Chapman RD. Synth Commun 2003;33:2677.

412. Berkessel A, Glaubitz K, Lex J. Eur J Org Chem 2002:2948.

413. Pelter A, Elgendy SMA. J Chem Soc, Perkin Trans 1 1993:1891.

414. Kurti L, Herczegh P, Visy J, Simonyi M, Antus S, Pelter A. J Chem Soc, Perkin Trans 1 1999:379.

415. McKillop A, McLaren L, Taylor RJK. J Chem Soc, Perkin Trans 1 1994:2047.

416. Mitchell AS, Russell RA. Tetrahedron Lett 1993;34:545.

417. Lion CJ, Vasselin DA, Schwalbe CH, Matthews CS, Stevens MFG, Westwell AD. Org Biomol Chem 2005;3:3996. [PubMed: 16240020]

418. Quideau S, Pouysegu L, Deffieux D, Ozanne A, Gagnepain J, Fabre I, Oxoby M. ARKIVOC 2003;vi: 106.

419. Karam O, Martin A, Jouannetaud M-P, Jacquesy J-C. Tetrahedron Lett 1999;40:4183.

420. Wipf P, Kim Y, Fritch PC. J Org Chem 1993;58:7195.

421. Quideau S, Looney MA, Pouysegu L, Ham S, Birney DM. Tetrahedron Lett 1999;40:615.

422. Kita Y, Tohma H, Kikuchi K, Inagaki M, Yakura T. J Org Chem 1991;56:435.

423. Murakata M, Yamada K, Hoshino O. Chem Commun 1994:443.

424. Kita Y, Tohma H, Inagaki M, Hatanaka K, Yakura T. J Am Chem Soc 1992;114:2175.

425. Berard D, Giroux M-A, Racicot L, Sabot C, Canesi S. Tetrahedron 2008;64:7537.

426. Basset L, Martin-Mingot A, Jouannetaud M-P, Jacquesy J-C. Tetrahedron Lett 2008;49:1551.

427. Karam O, Martin-Mingot A, Jouannetaud M-P, Jacquesy J-C, Cousson A. Tetrahedron 2004;60:6629.

428. Roy H, Sarkar M, Mal D. Synth Commun 2005;35:2183.

429. Dey S, Mal D. Tetrahedron Lett 2005;46:5483.

430. Venkateswarlu R, Kamakshi C, Subhash PV, Moinuddin SGA, Reddy DRS, Ward RS, Pelter A, Gelbrich T, Hursthouse MB, Coles SJ, Light ME. Tetrahedron 2006;62:4463.

431. Felpin F-X. Tetrahedron Lett 2007;48:409.

432. Quideau S, Looney MA, Pouysegu L. Org Lett 1999;1:1651.

433. Quideau S, Pouysegu L, Oxoby M, Looney MA. Tetrahedron 2001;57:319.

434. Lebrasseur N, Fan G-J, Quideau S. ARKIVOC 2004;xiii:5.

435. Lebrasseur N, Fan G-J, Oxoby M, Looney MA, Quideau S. Tetrahedron 2005;61:1551.

436. Braun NA, Ousmer M, Bray JD, Bouchu D, Peters K, Peters E-M, Ciufolini MA. J Org Chem 2000;65:4397. [PubMed: 10891144] 
437. Shigehisa H, Takayama J, Honda T. Tetrahedron Lett 2006;47:7301.

438. Quideau S, Lebon M, Lamidey A-M. Org Lett 2002;4:3975. [PubMed: 12599506]

439. Wipf P, Spencer SR. J Am Chem Soc 2005;127:225. [PubMed: 15631472]

440. Canesi S, Bouchu D, Ciufolini MA. Angew Chem, Int Ed 2004;43:4336.

441. Canesi S, Belmont P, Bouchu D, Rousset L, Ciufolini MA. Tetrahedron Lett 2002;43:5193.

442. Nicolaou KC, Edmonds DJ, Li A, Tria GS. Angew Chem, Int Ed 2007;46:3942.

443. Tohma H, Harayama Y, Hashizume M, Iwata M, Kiyono Y, Egi M, Kita Y. J Am Chem Soc 2003;125:11235. [PubMed: 16220942]

444. Baxendale IR, Ley SV, Nessi M, Piutti C. Tetrahedron 2002;58:6285.

445. Pouysegu L, Avellan A-V, Quideau S. J Org Chem 2002;67:3425. [PubMed: 12003555]

446. Quideau S, Pouysegu L, Avellan AV, Whelligan DK, Looney MA. Tetrahedron Lett 2001;42:7393.

447. Van De Water RW, Hoarau C, Pettus TRR. Tetrahedron Lett 2003;44:5109. [PubMed: 16957784]

448. Vo NT, Pace RDM, O’Hara F, Gaunt MJ. J Am Chem Soc 2008;130:404. [PubMed: 18081291]

449. Baxendale IR, Deeley J, Griffiths-Jones CM, Ley SV, Saaby S, Tranmer GK. Chem Commun 2006:2566.

450. Baxendale IR, Lee A-L, Ley SV. J Chem Soc, Perkin Trans 1 2002:1850.

451. Moisan L, Wagner M, Comesse S, Doris E. Tetrahedron Lett 2006;47:9093.

452. Dohi T, Maruyama A, Yoshimura M, Morimoto K, Tohma H, Kita Y. Angew Chem, Int Ed 2005; 44:6193.

453. Dohi T, Maruyama A, Minamitsuji Y, Takenaga N, Kita Y. Chem Commun 2007:1224.

454. Yakura T, Konishi T. Synlett 2007:765.

455. Dohi T, Maruyama A, Takenage N, Senami K, Minamitsuji Y, Fujioka H, Caemmerer S, Kita Y. Angew Chem, Int Ed 2008;47:3787.

456. Gagnepain J, Mereau R, Dejugnac D, Leger J-M, Castet F, Deffieux D, Pouysegu L, Quideau S. Tetrahedron 2007;63:6493.

457. Lai C-H, Lin P-Y, Peddinti RK, Liao C-C. Synlett 2002:1520.

458. Cook SP, Danishefsky SJ. Org Lett 2006;8:5693. [PubMed: 17134249]

459. Berube A, Drutu I, Wood JL. Org Lett 2006;8:5421. [PubMed: 17107037]

460. Chittimalla SK, Liao C-C. Synlett 2002:565.

461. Hou H-F, Peddinti RK, Liao C-C. Org Lett 2002;4:2477. [PubMed: 12123355]

462. Boldron C, Aromi G, Challa G, Gamez P, Reedijk J. Chem Commun 2005:5808.

463. Hata K, Hamamoto H, Shiozaki Y, Caemmerer SB, Kita Y. Tetrahedron 2007;63:4052.

464. Kita Y, Tohma H, Hatanaka K, Takada T, Fujita S, Mitoh S, Sakurai H, Oka S. J Am Chem Soc 1994;116:3684.

465. Takada T, Arisawa M, Gyoten M, Hamada R, Tohma H, Kita Y. J Org Chem 1998;63:7698.

466. Hamamoto H, Anilkumar G, Tohma H, Kita Y. Chem Commun 2002:450.

467. Hamamoto H, Anilkumar G, Tohma H, Kita Y. Chem Eur J 2002;8:5377.

468. Pingaew R, Ruchirawat S. Synlett 2007:2363.

469. Taylor SR, Ung AT, Pyne SG, Skelton BW, White AH. Tetrahedron 2007;63:11377.

470. Moreno I, Tellitu I, Dominguez E, SanMartin R. Eur J Org Chem 2002:2126.

471. Dohi T, Ito M, Morimoto K, Iwata M, Kita Y. Angew Chem, Int Ed 2008;47:1301.

472. Besong G, Jarowicki K, Kocienski PJ, Sliwinski E, Boyle FT. Org Biomol Chem 2006;4:2193. [PubMed: 16729129]

473. Hata K, Hamamoto H, Shiozaki Y, Kita Y. Chem Commun 2005:2465.

474. Hamamoto H, Shiozaki Y, Hata K, Tohma H, Kita Y. Chem Pharm Bull 2004;52:1231. [PubMed: 15467242]

475. Huang W-J, Singh OV, Chen C-H, Lee S-S. Helv Chim Acta 2004;87:167.

476. Kita Y, Egi M, Okajima A, Ohtsubo M, Takada T, Tohma H. J Chem Soc, Chem Commun 1996:1491.

477. Kita Y, Egi M, Ohtsubo M, Saiki T, Takada T, Tohma H. J Chem Soc, Chem Commun 1996:2225. 
478. Hamamoto H, Hata K, Nambu H, Shiozaki Y, Tohma H, Kita Y. Tetrahedron Lett 2004;45:2293. 479. Hamamoto H, Shiozaki Y, Nambu H, Hata K, Tohma H, Kita Y. Chem Eur J 2004;10:4977.

480. Dohi T, Minamitsuji Y, Maruyama A, Hirose S, Kita Y. Org Lett 2008;10:3559. [PubMed: 18616338]

481. Tohma H, Iwata M, Maegawa T, Kita Y. Tetrahedron Lett 2002;43:9241.

482. Mirk D, Willner A, Froehlich R, Waldvogel SR. Adv Synth Catal 2004;346:675.

483. Jean A, Cantat J, Berard D, Bouchu D, Canesi S. Org Lett 2007;9:2553. [PubMed: 17530765]

484. Dohi T, Morimoto K, Kiyono Y, Maruyama A, Tohma H, Kita Y. Chem Commun 2005:2930.

485. Tohma H, Iwata M, Maegawa T, Kiyono Y, Maruyama A, Kita Y. Org Biomol Chem 2003;1:1647. [PubMed: 12926350]

486. Dohi T, Morimoto K, Maruyama A, Kita Y. Org Lett 2006;8:2007. [PubMed: 16671768]

487. Francisco CG, Gonzalez Martin C, Suarez E. J Org Chem 1998;63:2099.

488. Dorta RL, Martin A, Salazar JA, Suarez E, Prange T. J Org Chem 1998;63:2251.

489. Francisco CG, Gonzalez Martin C, Suarez E. J Org Chem 1998;63:8092.

490. Francisco CG, Gonzalez CC, Paz NR, Suarez E. Org Lett 2003;5:4171. [PubMed: 14572277]

491. Alonso-Cruz CR, Kennedy AR, Rodriguez MS, Suarez E. Org Lett 2003;5:3729. [PubMed: 14507216]

492. Alonso-Cruz CR, Kennedy AR, Rodriguez MS, Suarez E. Tetrahedron Lett 2007;48:7207.

493. Alonso-Cruz CR, Leon EI, Ortiz-Lopez FJ, Rodriguez MS, Suarez E. Tetrahedron Lett 2005;46:5265.

494. Francisco CG, Herrera AJ, Kennedy AR, Melian D, Suarez E. Ang Chem, Int Ed 2002;41:856.

495. Francisco CG, Freire R, Herrera AJ, Perez-Martin I, Suarez E. Tetrahedron 2007;63:8910.

496. Francisco CG, Freire R, Herrera AJ, Perez-Martin I, Suarez E. Org Lett 2002;4:1959. [PubMed: 12027657]

497. Martin A, Quintanal LM, Suarez E. Tetrahedron Lett 2007;48:5507.

498. Boto A, Hernandez D, Hernandez R. Org Lett 2007;9:1721. [PubMed: 17397175]

499. Boto A, Hernandez D, Hernandez R, Suarez E. Org Lett 2004;6:3785. [PubMed: 15469349]

500. Chen J-M, Huang X. Synthesis 2004:2459.

501. Antunes CSA, Bietti M, Lanzalunga O, Salamone M. J Org Chem 2004;69:5281. [PubMed: 15287771]

502. Boto A, Hernandez R, Montoya A, Suarez E. Tetrahedron Lett 2002;43:8269.

503. Boto A, Gallardo JA, Hernandez R, Saavedra CJ. Tetrahedron Lett 2005;46:7807.

504. Boto A, Hernandez R, De Leon Y, Murguia JR, Rodriguez-Afonso A. Tetrahedron Lett 2004;45:6841.

505. Iglesias-Arteaga MA, Avila-Ortiz CG, Juaristi E. Tetrahedron Lett 2002;43:5297.

506. Dohi T, Takenaga N, Goto A, Maruyama A, Kita Y. Org Lett 2007;9:3129. [PubMed: 17616204]

507. Barluenga J, Gonzalez-Bobes F, Gonzalez JM. Angew Chem, Int Ed 2002;41:2556.

508. Pradhan TK, Hassner A. Synthesis 2007:3361.

509. Martin A, Perez-Martin I, Suarez E. Org Lett 2005;7:2027. [PubMed: 15876046]

510. Francisco CG, Herrera AJ, Martin A, Perez-Martin I, Suarez E. Tetrahedron Lett 2007;48:6384.

511. Fan R, Pu D, Wen F, Wu J. J Org Chem 2007;72:8994. [PubMed: 17929871]

512. Momiyama N, Yamamoto Y, Yamamoto H. J Am Chem Soc 2007;129:1190. [PubMed: 17263400]

513. Singh CB, Ghosh H, Murru S, Patel BK. J Org Chem 2008;73:2924. [PubMed: 18318545]

514. But TYS, Toy PH. J Am Chem Soc 2006;128:9636. [PubMed: 16866510]

515. Kansara A, Sharma PK, Banerji KK. J Chem Res 2004:315.

516. ter Wiel MKJ, Vicario J, Davey SG, Meetsma A, Feringa BL. Org Biomol Chem 2005;3:28. [PubMed: 15602594]

517. Shang Z, Reiner J, Zhao K. Synth Commun 2006;36:1529.

518. Zhutov EV, Skornyakov YV, Proskurina MV, Zefirov NS. Russ J Org Chem 2003;39:1672.

519. Tohma H, Maegawa T, Kita Y. ARKIVOC 2003;vi:62. 
520. Burghardt TE. J Sulfur Chem 2005;26:411.

521. Wu Y, Shen X, Yang Y-Q, Hu Q, Huang J-H. J Org Chem 2004;69:3857. [PubMed: 15153019]

522. Wu Y, Shen X, Yang Y-Q, Hu Q, Huang J-H. Tetrahedron Lett 2004;45:199.

523. Fleming FF, Funk L, Altundas R, Tu Y. J Org Chem 2001;66:6502. [PubMed: 11559208]

524. Shi X-X, Wu Q-Q. Synth Commun 2000;30:4081.

525. Makowiec S, Rachon J. Heteroatom Chem 2003;14:352.

526. Young KJH, Mironov OA, Periana RA. Organometallics 2007;26:2137.

527. Li Z, Xia C-G. J Mol Catal A 2004;214:95.

528. In J-H, Park S-E, Song R, Nam W. Inorg Chim Acta 2003;343:373.

529. Yusubov MS, Chi K-W, Park JY, Karimov R, Zhdankin VV. Tetrahedron Lett 2006;47:6305.

530. Iwasa S, Morita K, Tajima K, Fakhruddin A, Nishiyama H. Chem Lett 2002:284.

531. Miyamura H, Akiyama R, Ishida T, Matsubara R, Takeuchi M, Kobayashi S. Tetrahedron 2005;61:12177.

532. Sun W, Wang H, Xia C, Li J, Zhao P. Angew Chem, Int Ed 2003;42:1042.

533. Li Z, Tang ZH, Hu XX, Xia CG. Chem Eur J 2005;11:1210.

534. Karimipour GR, Shadegan HA, Ahmadpour R. J Chem Res 2007:252.

535. Adam W, Hajra S, Herderich M, Saha-Moeller CR. Org Lett 2000;2:2773. [PubMed: 10964362]

536. Kunst E, Gallier F, Dujardin G, Yusubov MS, Kirschning A. Org Lett 2007;9:5199. [PubMed: 17999512]

537. Provins L, Murahashi S-I. ARKIVOC 2007;x:107.

538. Dick AR, Hull KL, Sanford MS. J Am Chem Soc 2004;126:2300. [PubMed: 14982422]

539. Alexanian EJ, Lee C, Sorensen EJ. J Am Chem Soc 2005;127:7690. [PubMed: 15913354]

540. Desai LV, Hull KL, Sanford MS. J Am Chem Soc 2004;126:9542. [PubMed: 15291549]

541. Kalyani D, Sanford MS. Org Lett 2005;7:4149. [PubMed: 16146374]

542. Kalyani D, Dick AR, Anani WQ, Sanford MS. Org Lett 2006;8:2523. [PubMed: 16737304]

543. Wang D-H, Hao X-S, Wu D-F, Yu J-Q. Org Lett 2006;8:3387. [PubMed: 16836412]

544. Welbes LL, Lyons TW, Cychosz KA, Sanford MS. J Am Chem Soc 2007;129:5836. [PubMed: 17432861]

545. Desai LV, Sanford MS. Angew Chem, Int Ed 2007;46:5737.

546. Liu G, Stahl SS. J Am Chem Soc 2006;128:7179. [PubMed: 16734468]

547. Streuff J, Hoevelmann CH, Nieger M, Muniz K. J Am Chem Soc 2005;127:14586. [PubMed: 16231907]

548. Kalberer EW, Whitfield SR, Sanford MS. J Mol Catal A 2006;251:108.

549. Dick AR, Kampf JW, Sanford MS. Organometallics 2005;24:482.

550. Muniz K, Hoevelmann CH, Streuff J. J Am Chem Soc 2008;130:763. [PubMed: 18081279]

551. Giri R, Chen X, Yu J-Q. Angew Chem, Int Ed 2005;44:2112.

552. Daugulis O, Zaitsev VG. Angew Chem, Int Ed 2005;44:4046.

553. Dick AR, Kampf JW, Sanford MS. J Am Chem Soc 2005;127:12790. [PubMed: 16159259]

554. Yan J, Wu J, Jin H. J Organomet Chem 2007;692:3636.

555. Yan J, Lin F, Yang Z. Synthesis 2007:1301.

556. Zefirov NS, Sorokin VD, Zhdankin VV, Koz'min AS. Russ J Org Chem 1986;22:450.

557. Bassindale AR, Katampe I, Taylor PG. Can J Chem 2000;78:1479.

558. Robinson RI, Woodward S. Tetrahedron Lett 2003;44:1655.

559. Koser GF, Wettach RH. J Org Chem 1977;42:1476.

560. Nabana T, Togo H. J Org Chem 2002;67:4362. [PubMed: 12054975]

561. Yamamoto Y, Togo H. Synlett 2005:2486.

562. Hatzigrigoriou E, Varvoglis A, Bakola-Christianopoulou M. J Org Chem 1990;55:315.

563. Yusubov MS, Wirth T. Org Lett 2005;7:519. [PubMed: 15673279]

564. Abe S, Sakuratani K, Togo H. Synlett 2001:22.

Chem Rev. Author manuscript; available in PMC 2009 December 10. 
565. Abe S, Sakuratani K, Togo H. J Org Chem 2001;66:6174. [PubMed: 11529748]

566. Zefirov NS, Zhdankin VV, Dan'kov YV, Sorokin VD, Semerikov VN, Koz'min AS, Caple R, Berglund BA. Tetrahedron Lett 1986;27:3971.

567. Hembre RT, Scott CP, Norton JR. J Org Chem 1987;52:3650.

568. Zhdankin VV, Crittell CM, Stang PJ. Tetrahedron Lett 1990;31:4821.

569. Kitamura T, Inoue D, Wakimoto I, Nakamura T, Katsuno R, Fujiwara Y. Tetrahedron 2004;60:8855.

570. Koser GF, Wettach RH, Troup JM, Frenz BA. J Org Chem 1976;41:3609.

571. Wessig P, Muhling O. Helv Chim Acta 2003;86:865.

572. Kumar D, Sundaree S, Rao VS. Synth Commun 2006;36:1893.

573. Prakash O, Pannu K, Prakash R, Batra A. Molecules 2006;11:523. [PubMed: 17971723]

574. Kumar D, Sundaree S, Rao VS, Varma RS. Tetrahedron Lett 2006;47:4197.

575. Kumar D, Sundaree MS, Patel G, Rao VS, Varma RS. Tetrahedron Lett 2006;47:8239.

576. Xie Y-Y, Chen Z-C. Synth Commun 2002;32:1875.

577. Wan D-B, Chen J-M. J Chem Res 2006:32.

578. Prakash O, Batra A, Chaudhri V, Prakash R. Tetrahedron Lett 2005;46:2877.

579. Lee JC, Ku CH. Synlett 2002:1679.

580. Xie Y-Y. Synth Commun 2005;35:1741.

581. Handy ST, Okello M. Synlett 2002:489.

582. Aggarwal R, Sumran G. Synth Commun 2006;36:875.

583. Aggarwal R, Pundeer R, Kumar V, Chaudhri V, Singh SP, Prakash O. Synth Commun 2004;34:2659.

584. Lee JC, Seo J-W, Baek JW. Synth Commun 2007;37:2159.

585. Lee JC, Choi HJ, Lee YC. Tetrahedron Lett 2003;44:123.

586. Kawano Y, Togo H. Synlett 2008:217.

587. Muthukrishnan M, Patil PS, More SV, Joshi RA. Mendeleev Commun 2005:100.

588. Kawamura Y, Maruyama M, Tokuoka T, Tsukayama M. Synthesis 2002:2490.

589. Patonay T, Levai A, Riman E, Varma RS. ARKIVOC 2004;vii:183.

590. Karade NN, Gampawar SV, Kondre JM, Shinde SV. Tetrahedron Lett 2008;49:4402.

591. Li M, Zhao G, Wen L, Cao W, Zhang S, Yang H. J Heterocycl Chem 2005;42:209.

592. Li M, Zhao G, Wen L, Yang H. Synth Commun 2005;35:493.

593. Hou RS, Wang HM, Huang HY, Chen LC. Org Prep Proced Int 2004;36:491.

594. Lee JC, Park H-J, Park JY. Tetrahedron Lett 2002;43:5661.

595. Xie YY, Chen ZC, Zheng QG. J Chem Res (S) 2002:618.

596. Xie Y-Y, Chen Z-C, Zheng Q-G. Synthesis 2002:1505.

597. Hou R-S, Wang H-M, Lin Y-C, Chen L-C. Heterocycles 2005;65:649.

598. Richardson RD, Page TK, Altermann S, Paradine SM, French AN, Wirth T. Synlett 2007:538.

599. Yamamoto Y, Togo H. Synlett 2006:798.

600. Akiike J, Yamamoto Y, Togo H. Synlett 2007:2168.

601. Yamamoto Y, Kawano Y, Toy PH, Togo H. Tetrahedron 2007;63:4680.

602. Justik MW, Koser GF. Tetrahedron Lett 2004;45:6159.

603. Hossain MM, Tokuoka T, Yamashita K, Kawamura Y, Tsukayama M. Synth Commun 2006;36:1201.

604. Silva LF Jr, Siqueira FA, Pedrozo EC, Vieira FYM, Doriguetto AC. Org Lett 2007;9:1433. [PubMed: 17371034]

605. Prakash O, Kumar A, Sadana AK, Singh SP. Synthesis 2006:21.

606. Justik MW, Koser GF. Molecules 2005;10:217. [PubMed: 18007289]

607. Silva LF Jr, Vasconcelos RS, Nogueira MA. Org Lett 2008;10:1017. [PubMed: 18269290]

608. Koser GF, Telu S, Laali KK. Tetrahedron Lett 2006;47:7011.

609. Comins DL, Kuethe JT, Miller TM, Fevrier FC, Brooks CA. J Org Chem 2005;70:5221. [PubMed: 15960527] 
610. Chen J-M, Huang X. Synthesis 2004:1577.

611. Miyazawa E, Sakamoto T, Kikugawa Y. J Org Chem 2003;68:5429. [PubMed: 12816516]

612. Misu Y, Togo H. Org Biomol Chem 2003;1:1342. [PubMed: 12929664]

613. Walker SJ, Hart DJ. Tetrahedron Lett 2007;48:6214.

614. Lazbin IM, Koser GF. J Org Chem 1986;51:2669.

615. Vasudevan A, Koser GF. J Org Chem 1988;53:5158.

616. Moriarty RM, Khosrowshahi JS, Awasthi AK, Penmasta R. Synth Commun 1988;18:1179.

617. Liu SJ, Zhang JZ, Tian GR, Liu P. Synth Commun 2005;36:823.

618. Lee JC, Lee JY, Lee SJ. Tetrahedron Lett 2004;45:4939.

619. Sridhar PR, Prabhu KR, Chandrasekaran S. Eur J Org Chem 2004:4809.

620. Lee J, Lee J. Synth Commun 2006;36:1071.

621. Yan J, Zhou Z, Zhu M. Tetrahedron Lett 2005;46:8173.

622. Yan J. J Chem Res 2006:459.

623. Yan J, Zhu M, Zhou Z. Eur J Org Chem 2006:2060.

624. Hadjiarapoglou L, Spyroudis S, Varvoglis A. Synthesis 1983:207.

625. Papadopoulou M, Varvoglis A. J Chem Res (S) 1983:66.

626. Papadopoulou M, Varvoglis A. J Chem Res (S) 1984:166.

627. Zhdankin VV, Krasutsky AP, Kuehl CJ, Simonsen AJ, Woodward JK, Mismash B, Bolz JT. J Am Chem Soc 1996;118:5192.

628. Magnus P, Lacour J, Evans PA, Roe MB, Hulme C. J Am Chem Soc 1996;118:3406.

629. Magnus P, Lacour J, Weber W. J Am Chem Soc 1993;115:9347.

630. Magnus P, Lacour J, Weber W. Synthesis 1998:547.

631. Pedersen CM, Marinescu LG, Bols M. Org Biomol Chem 2005;3:816. [PubMed: 15731868]

632. Chung R, Yu E, Incarvito CD, Austin DJ. Org Lett 2004;6:3881. [PubMed: 15496054]

633. Kirschning A, Abul Hashem M, Monenschein H, Rose L, Schoening K-U. J Org Chem 1999;64:6522.

634. Chen J-M, Huang X. Synth Commun 2004;34:1745.

635. Umemoto T, Kuriu Y, Shuyama H, Miyano O, Nakayama S. J Fluorine Chem 1986;31:37.

636. Umemoto T, Kuriu Y, Shuyama H, Miyano O, Nakayama S. J Fluorine Chem 1982;20:695.

637. Tesevic V, Gladysz JA. Green Chem 2005;7:833.

638. Tesevic V, Gladysz JA. J Org Chem 2006;71:7433. [PubMed: 16958539]

639. Yagupol'skii LM, Maletina II, Kondratenko NV, Orda VV. Synthesis 1978:835.

640. Kuehl CJ, Bolz JT, Zhdankin VV. Synthesis 1995:312.

641. Zhdankin VV, Kuehl C. Tetrahedron Lett 1994;35:1809.

642. Umemoto T, Goto Y. Bull Chem Soc Jpn 1987;60:3307.

643. Zhdankin VV, Kuehl CJ, Simonsen AJ. Tetrahedron Lett 1995;36:2203.

644. Zhdankin VV, Kuehl CJ, Simonsen AJ. J Org Chem 1996;61:8272. [PubMed: 11667815]

645. Zhdankin VV, Erickson SA, Hanson KJ. J Am Chem Soc 1997;119:4775.

646. Minkwitz R, Berkei M. Inorg Chem 1999;38:5041. [PubMed: 11671248]

647. Minkwitz R, Berkei M. Inorg Chem 2001;40:36. [PubMed: 11195384]

648. Minkwitz R, Berkei M. Z Naturforsch, B: Chem Sci 2000;55:718.

649. Minkwitz R, Berkei M. Z Anorg Allg Chem 2000;626:2325.

650. Minkwitz R, Berkei M, Ludwig R. Eur J Inorg Chem 2000:2387.

651. DesMarteau DD, Montanari V. J Chem Soc, Chem Commun 1998:2241.

652. Meyer V, Wachter W. Ber 1892;25:2632.

653. Willgerodt C. J Prakt Chem 1894;49:466.

654. Wolf W, Steinberg L. J Chem Soc, Chem Commun 1965:449.

655. Zhdankin VV, Arbit RM, McSherry M, Mismash B, Young VG. J Am Chem Soc 1997;119:7408.

656. Amey RL, Martin JC. J Org Chem 1979;44:1779.

Chem Rev. Author manuscript; available in PMC 2009 December 10. 
657. Ochiai M, Masaki Y, Shiro M. J Org Chem 1991;56:5511.

658. Shefter E, Wolf W. J Pharm Sci 1965;54:104. [PubMed: 14288470]

659. Etter MC. J Am Chem Soc 1976;98:5326.

660. Etter MC. J Am Chem Soc 1976;98:5331.

661. Gougoutas JZ, Lessinger L. J Solid State Chem 1974;9:155.

662. Ochiai M, Ito T, Masaki Y, Shiro M. J Am Chem Soc 1992;114:6269.

663. Ochiai M, Ito T, Shiro M. J Chem Soc, Chem Commun 1993:218.

664. Zhdankin VV, Kuehl CJ, Arif AM, Stang PJ. Mendeleev Commun 1996:50.

665. Akai S, Okuno T, Takada T, Tohma H, Kita Y. Heterocycles 1996;42:47.

666. Braddock DC, Cansell G, Hermitage SA, White AJP. Chem Commun 2006:1442.

667. Eisenberger P, Gischig S, Togni A. Chem Eur J 2006;12:2579.

668. Kieltsch I, Eisenberger P, Togni A. Angew Chem, Int Ed 2007;46:754.

669. Koser, GF. The Chemistry of Functional Groups, Suppl D: Chem Halides, Pseudo-Halides, Azides. Patai, S.; Rappoport, Z., editors. Wiley-Interscience; Chichester: 1983.

670. Sueda T, Fukuda S, Ochiai M. Org Lett 2001;3:2387. [PubMed: 11463323]

671. Ochiai M, Ito T, Takahashi H, Nakanishi A, Toyonari M, Sueda T, Goto S, Shiro M. J Am Chem Soc 1996;118:7716.

672. Dolenc D, Plesnicar B. J Am Chem Soc 1997;119:2628.

673. Ochiai M, Nakanishi A, Ito T. J Org Chem 1997;62:4253. [PubMed: 11671744]

674. Ochiai M, Sueda T. Tetrahedron Lett 2004;45:3557.

675. Krasutsky AP, Kuehl CJ, Zhdankin VV. Synlett 1995:1081.

676. Zhdankin VV, Kuehl CJ, Krasutsky AP, Formaneck MS, Bolz JT. Tetrahedron Lett 1994;35:9677.

677. Zhdankin VV, Kuehl CJ, Krasutsky AP, Bolz JT, Mismash B, Woodward JK, Simonsen AJ.

Tetrahedron Lett 1995;36:7975.

678. Sueda T, Takeuchi Y, Suefuji T, Ochiai M. Molecules 2005;10:195. [PubMed: 18007287]

679. Eisenberger P, Kieltsch I, Armanino N, Togni A. Chem Commun 2008:1575.

680. Zhang W, Zhu J, Hu J. Tetrahedron Lett 2008;49:5006.

681. Okuyama T, Takino T, Sueda T, Ochiai M. J Am Chem Soc 1995;117:3360.

682. Shirai A, Kubo H, Takahashi E. J Photopolym Sci Technol 2002;15:29.

683. VanderHart DL, Prabhu VM, Lin EK. Chem Mater 2004;16:3074.

684. Slegt M, Minne F, Zuilhof H, Overkleeft HS, Lodder G. Eur J Org Chem 2007:5353.

685. Tasdelen MA, Kumbaraci V, Jockusch S, Turro NJ, Talinli N, Yagci Y. Macromolecules 2008;41:295.

686. Goldstein EJC, Citron DM, Warren Y, Merriam CV, Tyrrell K, Fernandez H, Radhakrishnan U, Stang PJ, Conrads G. Antimicrobial Agents and Chemotherapy 2004;48:2766. [PubMed: 15215147]

687. Montanari V, Kumar K. J Am Chem Soc 2004;126:9528. [PubMed: 15291542]

688. DesMarteau DD, Montanari V. Chem Lett 2000:1052.

689. DesMarteau DD, Montanari V. J Fluorine Chem 2001;109:19.

690. Montanari V, Kumar K. J Fluorine Chem 2006;127:565.

691. Montanari V, Kumar K. Eur J Org Chem 2006:874.

692. Liu CY, Li H, Meng AG. Acta Crystallogr, Sect E: Struct Rep 2007;E63:o3647.

693. DesMarteau DD, Pennington WT, Montanari V, Thomas BH. J Fluorine Chem 2003;122:57.

694. Kaafarani BR, Gu H, Pinkerton AA, Neckers DC. J Chem Soc, Dalton Trans 2002:2318.

695. Bykowski D, McDonald R, Hinkle RJ, Tykwinski RR. J Org Chem 2002;67:2798. [PubMed: 11975530]

696. Ochiai M, Toyonari M, Sueda T, Kitagawa Y. Tetrahedron Lett 1996;37:8421.

697. Chen D-W, Ochiai M. J Org Chem 1999;64:6804. [PubMed: 11674690]

698. Kalyani D, Deprez NR, Desai LV, Sanford MS. J Am Chem Soc 2005;127:7330. [PubMed: 15898779] 
699. Ochiai M, Ito T, Takaoka Y, Masaki Y. J Am Chem Soc 1991;113:1319.

700. Helber J, Frohn H-J, Klose A, Scholten T. ARKIVOC 2003;vi:71.

701. Dohi T, Ito M, Morimoto K, Minamitsuji Y, Takenaga N, Kita Y. Chem Commun 2007:4152.

702. Koser GF, Wettach RH. J Org Chem 1980;45:1542.

703. Carman CS, Koser GF. J Org Chem 1983;48:2534.

704. Pike VW, Butt F, Shah A, Widdowson DA. J Chem Soc, Perkin Trans 1 1999:245.

705. Zhang M-R, Kumata K, Suzuki K. Tetrahedron Lett 2007;48:8632.

706. Carroll MA, Pike VW, Widdowson DA. Tetrahedron Lett 2000;41:5393.

707. Kitamura T, Matsuyuki J, Nagata K, Furuki R, Taniguchi H. Synthesis 1992:945.

708. Shah A, Pike VW, Widdowson DA. J Chem Soc, Perkin Trans 1 1997:2463.

709. Hossain MD, Kitamura T. Tetrahedron 2006;62:6955.

710. Hossain MD, Ikegami Y, Kitamura T. J Org Chem 2006;71:9903. [PubMed: 17168620]

711. Hossain MD, Kitamura T. Bull Chem Soc Jpn 2007;80:2213.

712. Bielawski M, Zhu M, Olofsson B. Adv Synth Catal 2007;349:2610.

713. Bielawski M, Olofsson B. J Chem Soc, Chem Commun 2007:2521.

714. Zhu M, Jalalian N, Olofsson B. Synlett 2008:592.

715. Bielawski M, Aili D, Olofsson B. J Org Chem 2008;73:4602. [PubMed: 18505294]

716. Kraszkiewicz L, Skulski L. Synthesis 2008:2373.

717. Stang PJ, Tykwinski R, Zhdankin V. J Heterocycl Chem 1992;29:815.

718. Stang PJ, Zhdankin VV, Tykwinski R, Zefirov NS. Tetrahedron Lett 1992;33:1419.

719. Radhakrishnan U, Stang PJ. J Org Chem 2003;68:9209. [PubMed: 14629137]

720. Stang PJ, Olenyuk B, Chen K. Synthesis 1995:937.

721. Aggarwal VK, Olofsson B. Angew Chem, Int Ed 2005;44:5516.

722. Beringer FM, Nathan RA. J Org Chem 1970;35:2095.

723. Beringer FM, Nathan RA. J Org Chem 1969;34:685.

724. Stang PJ, Chen K. J Am Chem Soc 1995;117:1667.

725. Wang F-Y, Chen Z-C, Zheng Q-G. J Chem Res (S) 2003:620.

726. Wang F-Y, Chen Z-C, Zheng Q-G. J Chem Res (S) 2003:810.

727. Wang F-Y, Chen Z-C, Zheng Q-G. J Chem Res 2004:206.

728. Carroll MA, Wood RA. Tetrahedron 2007;63:11349.

729. Yan J, Hu W, Rao G. Synthesis 2006:943.

730. Xue Z, Yang D, Wang C. J Organomet Chem 2006;691:247.

731. Fujita M, Mishima E, Okuyama T. J Phys Org Chem 2007;20:241.

732. Ermert J, Hocke C, Ludwig T, Gail R, Coenen HH. J Labelled Compd Radiopharm 2004;47:429.

733. Oh CH, Kim JS, Jung HH. J Org Chem 1999;64:1338.

734. Ochiai M, Kitagawa Y, Takayama N, Takaoka Y, Shiro M. J Am Chem Soc 1999;121:9233.

735. Ozanne-Beaudenon A, Quideau S. Angew Chem, Int Ed 2005;44:7065.

736. Quideau S, Pouysegu L, Ozanne A, Gagnepain J. Molecules 2005;10:201. [PubMed: 18007288]

737. Marsh G, Stenutz R, Bergman A. Eur J Org Chem 2003:2566.

738. Couladouros EA, Moutsos VI, Pitsinos EN. ARKIVOC 2003;xv:92.

739. Ryan JH, Stang PJ. Tetrahedron Lett 1997;38:5061.

740. Zhang B-X, Nuka T, Fujiwara Y, Yamaji T, Hou Z, Kitamura T. Heterocycles 2004;64:199.

741. Zhou T, Chen Z-C. J Chem Res 2004:404.

742. Zhou T, Li T-C, Chen Z-C. Helv Chim Acta 2005;88:290.

743. Kang S-K, Lee H-W, Jang S-B, Ho P-S. J Org Chem 1996;61:4720. [PubMed: 11667403]

744. Bumagin NA, Tsarev DA. Tetrahedron Lett 1998;39:8155.

745. Al-Qahtani MH, Pike VW. J Chem Soc, Perkin Trans 1 2000:1033.

746. Kang S-K, Yamaguchi T, Ho P-S, Kim W-Y, Yoon S-K. Tetrahedron Lett 1997;38:1947.

747. Kang S-K, Choi S-C, Baik T-G. Synth Commun 1999;29:2493. 
748. Kang S-K, Ryu H-C, Kim J-W. Synth Commun 2001;31:1021.

749. Kang S-K, Yamaguchi T, Ho P-S, Kim W-Y, Ryu H-C. J Chem Soc, Perkin Trans 1 1998:841.

750. Kang S-K, Lee H-W, Jang S-B, Kim T-H, Pyun S-J. J Org Chem 1996;61:2604. [PubMed: 11667088]

751. Kang S-K, Baik T-G, Hur Y. Tetrahedron 1999;55:6863.

752. Kang S-K, Ha Y-H, Yang H-Y. J Chem Res (S) 2002:282.

753. Wang L, Chen Z-C. Synth Commun 2000;30:3607.

754. Liang Y, Luo S, Liu C, Wu X, Ma Y. Tetrahedron 2000;56:2961.

755. Zhu M, Song Y, Cao Y. Synthesis 2007:853.

756. Radhakrishnan U, Stang PJ. Org Lett 2001;3:859. [PubMed: 11263900]

757. Becht J-M, Le Drian C. Org Lett 2008;10:3161. [PubMed: 18553969]

758. Deprez NR, Kalyani D, Krause A, Sanford MS. J Am Chem Soc 2006;128:4972. [PubMed: 16608329]

759. Canty AJ, Patel J, Rodemann T, Ryan JH, Skelton BW, White AH. Organometallics 2004;23:3466.

760. Kitamura T, Yamane M, Inoue K, Todaka M, Fukatsu N, Meng Z, Fujiwara Y. J Am Chem Soc 1999;121:11674.

761. Kitamura T, Meng Z, Fujiwara Y. Tetrahedron Lett 2000;41:6611.

762. Kitamura T, Wasai K, Todaka M, Fujiwara Y. Synlett 1999:731.

763. Kitamura T, Aoki Y, Isshiki S, Wasai K, Fujiwara Y. Tetrahedron Lett 2006;47:1709.

764. Kitamura T, Abe T, Fujiwara Y, Yamaji T. Synthesis 2003:213.

765. Xue J, Huang X. Synth Commun 2007;37:2179.

766. Kamila S, Koh B, Biehl ER. Synth Commun 2006;36:3493.

767. Thiemann T, Fujii H, Ohira D, Arima K, Li Y, Mataka S. New J Chem 2003;27:1377.

768. Xue J, Yang Y, Huang X. Synlett 2007:1533.

769. Rao UN, Sathunuru R, Maguire JA, Biehl E. J Heterocycl Chem 2004;41:13.

770. Chen Y-L, Hau C-K, Wang H, He H, Wong M-S, Lee AWM. J Org Chem 2006;71:3512. [PubMed: 16626133]

771. Lee T, Jeon J, Song KH, Jung I, Baik C, Park K-M, Lee SS, Kang SO, Ko J. J Chem Soc, Dalton Trans 2004:933.

772. Ochiai M, Sumi K, Takaoka Y, Kunishima M, Nagao Y, Shiro M, Fujita E. Tetrahedron 1988;44:4095.

773. Ochiai M, Sueda T, Noda R, Shiro M. J Org Chem 1999;64:8563.

774. Ochiai M, Toyonari M, Nagaoka T, Chen D-W, Kida M. Tetrahedron Lett 1997;38:6709.

775. Fujita M, Lee HJ, Okuyama T. Org Lett 2006;8:1399. [PubMed: 16562901]

776. Hinkle RJ, Thomas DB. J Org Chem 1997;62:7534.

777. Pirguliyev NS, Brel VK, Akhmedov NG, Zefirov NS. Synthesis 2000:81.

778. Yoshida M, Hara S. Org Lett 2003;5:573. [PubMed: 12583772]

779. Yoshida M, Komata A, Hara S. Tetrahedron 2006;62:8636.

780. Hinkle RJ, Stang PJ. Synthesis 1994:313.

781. McNeil AJ, Hinkle RJ, Rouse EA, Thomas QA, Thomas DB. J Org Chem 2001;66:5556. [PubMed: 11485483]

782. Chen J-M, Huang X. Synlett 2004:552.

783. Zhang P, Chen Z. J Chem Res (S) 2003:570.

784. Okuyama T, Fujita M. Acc Chem Res 2005;38:679. [PubMed: 16104691]

785. Fujita M, Kim WH, Sakanishi Y, Fujiwara K, Hirayama S, Okuyama T, Ohki Y, Tatsumi K, Yoshioka Y. J Am Chem Soc 2004;126:7548. [PubMed: 15198602]

786. Fujita M, Sakanishi Y, Nishii M, Yamataka H, Okuyama T. J Org Chem 2002;67:8130. [PubMed: 12423142]

787. Fujita M, Kim WH, Fujiwara K, Okuyama T. J Org Chem 2005;70:480. [PubMed: 15651790]

788. Hinkle RJ, Mikowski AM. ARKIVOC 2003;vi:201.

789. Fujita M, Sakanishi Y, Nishii M, Okuyama T. J Org Chem 2002;67:8138. [PubMed: 12423143]

Chem Rev. Author manuscript; available in PMC 2009 December 10. 
790. Slegt M, Gronheid R, Van der Vlugt D, Ochiai M, Okuyama T, Zuilhof H, Overkleeft HS, Lodder G. J Org Chem 2006;71:2227. [PubMed: 16526767]

791. Okuyama T, Lodder G. Adv Phys Org Chem 2002;37:1.

792. Fujita M, Sakanishi Y, Kim WH, Okuyama T. Chem Lett 2002:908.

793. Fujita M, Ihara K, Kim WH, Okuyama T. Bull Chem Soc Jpn 2003;76:1849.

794. Yan J, Jin H, Chen Z. J Chem Res 2007:233.

795. Zhang P-F, Chen Z-C. J Chem Res (S) 2002:388.

796. Guan T, Yoshida M, Hara S. J Org Chem 2007;72:9617. [PubMed: 17979292]

797. Hara S, Guan T, Yoshida M. Org Lett 2006;8:2639. [PubMed: 16737333]

798. Ochiai M, Shu T, Nagaoka T, Kitagawa Y. J Org Chem 1997;62:2130. [PubMed: 11671518]

799. Kang S-K, Yamaguchi T, Kim T-H, Ho P-S. J Org Chem 1996;61:9082.

800. Huang X, Xu X-H. J Chem Soc, Perkin Trans 1 1998:3321.

801. Kang S-K, Yoon S-K, Kim Y-M. Org Lett 2001;3:2697. [PubMed: 11506612]

802. Thielges S, Bisseret P, Eustache J. Org Lett 2005;7:681. [PubMed: 15704924]

803. Moriarty RM, Epa WR, Awasthi AK. J Am Chem Soc 1991;113:6315.

804. Yoshida M, Hara S, Fukuhara T, Yoneda N. Tetrahedron Lett 2000;41:3887.

805. Yoshida M, Yoshikawa S, Fukuhara T, Yoneda N, Hara S. Tetrahedron 2001;57:7143.

806. Yoshida M, Komata A, Hara S. J Fluorine Chem 2004;125:527.

807. Guan T, Takemura K, Senboku H, Yoshida M, Hara S. Tetrahedron Lett 2007;49:76.

808. Ochiai M, Uemura K, Masaki Y. J Am Chem Soc 1993;115:2528.

809. Ochiai M, Kunishima M, Tani S, Nagao Y. J Am Chem Soc 1991;113:3135.

810. Feldman, KS. Strategies and Tactics in Organic Synthesis. Harmata, M., editor. Vol. 4. Elsevier; London: 2004.

811. Ochiai M, Kunishima M, Sumi K, Nagao Y, Fujita E, Arimoto M, Yamaguchi H. Tetrahedron Lett 1985;26:4501.

812. Rebrovic L, Koser GF. J Org Chem 1984;49:4700.

813. Koumbis AE, Kyzas CM, Savva A, Varvoglis A. Molecules 2005;10:1340. [PubMed: 18007528]

814. Yoshida M, Nishimura N, Hara S. Chem Commun 2002:1014.

815. Stang PJ, Surber BW, Chen ZC, Roberts KA, Anderson AG. J Am Chem Soc 1987;109:228.

816. Kitamura T, Lee CH, Taniguchi H, Matsumoto M, Sano Y. J Org Chem 1994;59:8053.

817. Stang PJ, Williamson BL, Zhdankin VV. J Am Chem Soc 1991;113:5870.

818. Feldman KS, Bruendl MM, Schildknegt K, Bohnstedt AC. J Org Chem 1996;61:5440.

819. Feldman KS, Saunders JC, Wrobleski ML. J Org Chem 2002;67:7096. [PubMed: 12354005]

820. Feldman KS, Perkins AL, Masters KM. J Org Chem 2004;69:7928. [PubMed: 15527272]

821. Wardrop DJ, Fritz J. Org Lett 2006;8:3659. [PubMed: 16898785]

822. Bachi MD, Bar-Ner N, Crittell CM, Stang PJ, Williamson BL. J Org Chem 1991;56:3912.

823. Stang PJ, Arif AM, Crittell CM. Angew Chem, Int Ed 1990;29:287.

824. Ochiai M, Ito T, Takaoka Y, Masaki Y, Kunishima M, Tani S, Nagao Y. J Chem Soc, Chem Commun 1990:118.

825. Stang PJ, Murch P. Synthesis 1997:1378.

826. Zhang J-L, Chen Z-C. Synth Commun 1997;27:3757.

827. Zhang J-L, Chen Z-C. Synth Commun 1997;27:3881.

828. Zhang J-L, Chen Z-C. Synth Commun 1998;28:175.

829. Kitamura T, Tashi N, Tsuda K, Chen H, Fujiwara Y. Heterocycles 2000;52:303.

830. Kerwin SM, Nadipuram A. Synlett 2004:1404.

831. Witulski B, Stengel T. Angew Chem, Int Ed 1999;38:2426.

832. Hashmi ASK, Salathe R, Frey W. Synlett 2007:1763.

833. Martinez-Esperon MF, Rodriguez D, Castedo L, Saa C. Org Lett 2005;7:2213. [PubMed: 15901172] 834. Bykowski D, McDonald R, Tykwinski RR. ARKIVOC 2003;vi:21. 
835. Canty AJ, Rodemann T, Skelton BW, White AH. Organometallics 2006;25:3996.

836. Chaudhuri PD, Guo R, Malinakova HC. J Organomet Chem 2008;693:567.

837. Canty AJ, Rodemann T. Inorg Chem Commun 2003;6:1382.

838. Canty AJ, Watson RP, Karpiniec SS, Rodemann T, Gardiner MG, Jones RC. Organometallics 2008;27:3203.

839. Kitamura T, Lee CH, Taniguchi Y, Fujiwara Y, Matsumoto M, Sano Y. J Am Chem Soc 1997;119:619.

840. Yang D-Y, He J, Miao S. Synth Commun 2003;33:2695.

841. Yu C-M, Kweon J-H, Ho P-S, Kang S-C, Lee GY. Synlett 2005:2631.

842. Liu Z, Chen Z-C, Zheng Q-G. J Chem Res (S) 2003:715.

843. Liu Z, Chen Z-C, Zheng Q-G. J Heteterocycl Chem 2003;40:909.

844. Liu Z, Chen Z-C, Zheng Q-G. Synth Commun 2004;34:361.

845. Miyamoto K, Nishi Y, Ochiai M. Angew Chem, Int Ed 2005;44:6896.

846. Wipf P, Venkatraman S. J Org Chem 1996;61:8004. [PubMed: 11667780]

847. Gudriniece E, Neiland O, Vanags G. Zh Obshch Khim 1957;27:2737.

848. Hatjiarapoglou L, Varvoglis A, Alcock NW, Pike GA. J Chem Soc, Perkin Trans 1 1988:2839.

849. Mueller P, Fernandez D. Helv Chim Acta 1995;78:947.

850. Hadjiarapoglou L, Spyroudis S, Varvoglis A. J Am Chem Soc 1985; 107:7178.

851. Alcock NW, Bozopoulos AP, Hatzigrigoriou E, Varvoglis A. Acta Crystallogr, Sect C: Cryst Struct Commun 1990;C46:1300.

852. Moriarty RM, Prakash I, Prakash O, Freeman WA. J Am Chem Soc 1984;106:6082.

853. Matveeva ED, Podrugina TA, Grishin YK, Tkachev VV, Zhdankin VV, Aldoshin SM, Zefirov NS. Russ J Org Chem 2003;39:536.

854. Huang Z, Yu X, Huang X. J Org Chem 2002;67:8261. [PubMed: 12423166]

855. Yang R, Dai L, Chen C. J Chem Soc, Chem Commun 1992:1487.

856. Zhu S-Z. Heteroatom Chem 1994;5:9.

857. Ochiai M, Tada N, Okada T, Sota A, Miyamoto K. J Am Chem Soc 2008;130:2118. [PubMed: 18217752]

858. Ochiai M, Okada T, Tada N, Yoshimura A. Org Lett 2008;10:1425. [PubMed: 18303908]

859. Moriarty RM, Tyagi S, Ivanov D, Constantinescu M. J Am Chem Soc 2008;130:7564. [PubMed: 18491909]

860. Ochiai M, Kitagawa Y, Yamamoto S. J Am Chem Soc 1997;119:11598.

861. Ochiai M, Tuchimoto Y, Higashiura N. Org Lett 2004;6:1505. [PubMed: 15101778]

862. Ochiai M, Nishitani J, Nishi Y. J Org Chem 2002;67:4407. [PubMed: 12076135]

863. Bonge HT, Hansen T. Synlett 2007:55.

864. Bonge HT, Hansen T. Tetrahedron Lett 2008;49:57.

865. Moreau B, Charette AB. J Am Chem Soc 2005;127:18014. [PubMed: 16366547]

866. Wurz RP, Charette AB. Org Lett 2003;5:2327. [PubMed: 12816440]

867. Telu S, Durmus S, Koser GF. Tetrahedron Lett 2007;48:1863.

868. Gogonas EP, Nyxas I, Hadjiarapoglou LP. Synlett 2004:2563.

869. Malamidou-Xenikaki E, Spyroudis S, Tsanakopoulou M. J Org Chem 2003;68:5627. [PubMed: 12839455]

870. Spagou K, Malamidou-Xenikaki E, Spyroudis S. Molecules 2005;10:226. [PubMed: 18007290]

871. Koulouri S, Malamidou-Xenikaki E, Spyroudis S, Tsanakopoulou M. J Org Chem 2005;70:8780. [PubMed: 16238309]

872. Huang X-C, Liu Y-L, Liang Y, Pi S-F, Wang F, Li J-H. Org Lett 2008;10:1525. [PubMed: 18338899]

873. Gololobov YG, Golding IR, Galkina MA, Lokshin BV, Garbuzova IA, Petrovskii PV, Starikova ZA, Averkiev BB. Russ Chem Bull 2006;55:883.

874. Bakalbassis EG, Spyroudis S, Tsipis CA. Eur J Org Chem 2008:1783. 
875. Zhdankin VV, Maydanovych O, Herschbach J, Bruno J, Matveeva ED, Zefirov NS. J Org Chem 2003;68:1018. [PubMed: 12558430]

876. Zhdankin VV, Maydanovych O, Herschbach J, Bruno J, Matveeva ED, Zefirov NS. Tetrahedron Lett 2002;43:2359.

877. Huang Z-Z, Yu X-C, Huang X. Tetrahedron Lett 2002;43:6823.

878. Matveeva ED, Podrugina TA, Grishin YK, Pavlova AS, Zefirov NS. Russ J Org Chem 2007;43:201. 879. Deng G. J Chem Res (S) 2002:558.

880. Batsila C, Gogonas EP, Kostakis G, Hadjiarapoglou LP. Org Lett 2003;5:1511. [PubMed: 12713311]

881. Adam W, Gogonas EP, Hadjiarapoglou LP. Tetrahedron 2003;59:7929.

882. Adam W, Gogonas EP, Hadjiarapoglou LP. Eur J Org Chem 2003:1064.

883. Lee YR, Yoon SH, Seo Y, Kim BS. Synthesis 2004:2787.

884. Batsila C, Kostakis G, Hadjiarapoglou LP. Tetrahedron Lett 2002;43:5997.

885. Lee YR, Yoon SH. Synth Commun 2006;36:1941.

886. Lee YR, Jung YU. J Chem Soc, Perkin Trans 1 2002:1309.

887. Murphy GK, West FG. Org Lett 2006;8:4359. [PubMed: 16956226]

888. Adam W, Gogonas EP, Hadjiarapoglou LP. J Org Chem 2003;68:9155. [PubMed: 14604402]

889. Adam W, Gogonas EP, Hadjiarapoglou LP. Synlett 2003:1165.

890. Adam W, Gogonas EP, Nyxas IA, Hadjiarapoglou LP. Synthesis 2007:3211.

891. Adam W, Bosio SG, Gogonas EP, Hadjiarapoglou LP. Synthesis 2002:2084.

892. Ghanem A, Lacrampe F, Schurig V. Helv Chim Acta 2005;88:216.

893. Mueller P, Allenbach YF, Ferri M, Bernardinelli G. ARKIVOC 2003;vii:80.

894. Muller P, Bolea C. Helv Chim Acta 2002;85:483.

895. Zhu Q, Wu J, Fathi R, Yang Z. Org Lett 2002;4:3333. [PubMed: 12227782]

896. Yamada Y, Yamamoto T, Okawara M. Chem Lett 1975:361.

897. Sodergren MJ, Alonso DA, Bedekar AV, Andersson PG. Tetrahedron Lett 1997;38:6897.

898. Cicero RL, Zhao D, Protasiewicz JD. Inorg Chem 1996;35:275. [PubMed: 11666203]

899. Abramovitch RA, Bailey TD, Takaya T, Uma V. J Org Chem 1974;39:340.

900. Mansuy D, Mahy JP, Dureault A, Bedi G, Battioni P. J Chem Soc, Chem Commun 1984:1161.

901. Protasiewicz JD. Acta Crystallogr, Sect C: Cryst Struct Commun 1996;C52:1570.

902. Li J, Liang J-L, Chan PWH, Che C-M. Tetrahedron Lett 2004;45:2685.

903. Liang Y, Jiao L, Wang Y, Chen Y, Ma L, Xu J, Zhang S, Yu Z-X. Org Lett 2006;8:5877. [PubMed: 17134295]

904. Richardson RD, Desaize M, Wirth T. Chem Eur J 2007;13:6745.

905. Chang JWW, Chan PWH. Angew Chem, Int Ed 2008;47:1138.

906. Avenier F, Goure E, Dubourdeaux P, Seneque O, Oddou J-L, Pecaut J, Chardon-Noblat S, Deronzier A, Latour J-M. Angew Chem, Int Ed 2008;47:715.

907. Li Z, Capretto DA, Rahaman RO, He C. J Am Chem Soc 2007;129:12058. [PubMed: 17880068]

908. Li Z, Capretto DA, Rahaman R, He C. Angew Chem, Int Ed 2007;46:5184.

909. Cui Y, He C. Angew Chem, Int Ed 2004;43:4210.

910. He L, Yu J, Zhang J, Yu X-Q. Org Lett 2007;9:2277. [PubMed: 17497794]

911. Fiori KW, Du Bois J. J Am Chem Soc 2007;129:562. [PubMed: 17227019]

912. Fructos MR, Trofimenko S, Diaz-Requejo MM, Perez PJ. J Am Chem Soc 2006;128:11784.

[PubMed: 16953617]

913. Caselli A, Gallo E, Ragaini F, Oppezzo A, Cenini S. J Organomet Chem 2005;690:2142.

914. He L, Chan PWH, Tsui W-M, Yu W-Y, Che C-M. Org Lett 2004;6:2405. [PubMed: 15228290]

915. Jensen MP, Mehn MP, Que L Jr. Angew Chem, Int Ed 2003;42:4357.

916. Kohmura Y, Katsuki T. Tetrahedron Lett 2001;42:3339.

917. Evans DA, Bilodeau MT, Faul MM. J Am Chem Soc 1994;116:2742.

918. Li Z, Quan RW, Jacobsen EN. J Am Chem Soc 1995;117:5889. 
919. Li Z, Conser KR, Jacobsen EN. J Am Chem Soc 1993;115:5326.

920. Ma L, Du D-M, Xu J. Chirality 2006;18:575. [PubMed: 16642498]

921. Wang X, Ding K. Chem Eur J 2006;12:4568.

922. Ma L, Du D-M, Xu J. J Org Chem 2005;70:10155. [PubMed: 16292861]

923. Gullick J, Taylor S, Ryan D, McMorn P, Coogan M, Bethell D, Bulman Page PC, Hancock FE, King F, Hutchings GJ. Chem Commun 2003:2808.

924. Gillespie KM, Sanders CJ, O’Shaughnessy P, Westmoreland I, Thickitt CP, Scott P. J Org Chem 2002;67:3450. [PubMed: 12003558]

925. Anada M, Tanaka M, Washio T, Yamawaki M, Abe T, Hashimoto S. Org Lett 2007;9:4559. [PubMed: 17894504]

926. Tanaka M, Kurosaki Y, Washio T, Anada M, Hashimoto S. Tetrahedron Lett 2007;48:8799.

927. Liang J-L, Yuan S-X, Chan PWH, Che C-M. Org Lett 2002;4:4507. [PubMed: 12465924]

928. Okamura H, Bolm C. Org Lett 2004;6:1305. [PubMed: 15070323]

929. Knapp S, Yu Y. Org Lett 2007;9:1359. [PubMed: 17338541]

930. Guthikonda K, Wehn PM, Caliando BJ, Du Bois J. Tetrahedron 2006;62:11331.

931. Dick AR, Remy MS, Kampf JW, Sanford MS. Organometallics 2007;26:1365.

932. Mancheno OG, Bolm C. Org Lett 2006;8:2349. [PubMed: 16706523]

933. Mancheno OG, Bolm C. Chem Eur J 2007;13:6674.

934. Leca D, Song K, Amatore M, Fensterbank L, Lacote E, Malacria M. Chem Eur J 2004;10:906. 935. Archibald SJ, Boa AN, Pesa N. Chem Commun 2003:1736.

936. Jiang Y, Zhou G-C, He G-L, He L, Li J-L, Zheng S-L. Synthesis 2007:1459.

937. Jain SL, Sharma VB, Sain B. Tetrahedron Lett 2003;44:4385.

938. Wirth T. Angew Chem, Int Ed 2001;40:2812.

939. Kraszkiewicz L, Skulski L. ARKIVOC 2003;vi:120.

940. Koposov AY, Karimov RR, Pronin AA, Skrupskaya T, Yusubov MS, Zhdankin VV. J Org Chem 2006;71:9912. [PubMed: 17168623]

941. Alcock NW, Sawyer JF. J Chem Soc, Dalton Trans 1980:115.

942. Koposov AY, Zhdankin VV. Synthesis 2005:22.

943. Kuhakarn C, Kittigowittana K, Pohmakotr M, Reutrakul V. ARKIVOC 2005;i:143.

944. Chung W-J, Kim D-K, Lee Y-S. Tetrahedron Lett 2003;44:9251.

945. Kim D-K, Chung W-J, Lee Y-S. Synlett 2005:279.

946. Lecarpentier P, Crosignani S, Linclau B. Molecular Diversity 2005;9:341. [PubMed: 16311811]

947. Koposov AY, Litvinov DN, Zhdankin VV. Tetrahedron Lett 2004;45:2719.

948. Zhdankin V, Goncharenko RN, Litvinov DN, Koposov AY. ARKIVOC 2005;iv:8.

949. Ladziata U, Carlson J, Zhdankin VV. Tetrahedron Lett 2006;47:6301.

950. Ladziata U, Willging J, Zhdankin VV. Org Lett 2006;8:167. [PubMed: 16381594]

951. Karimov RR, Kazhkenov Z-GM, Modjewski MJ, Peterson EM, Zhdankin VV. J Org Chem 2007;72:8149. [PubMed: 17880245]

952. Hartman C, Mayer V. Chem Ber 1893;26:1727.

953. Gougoutas JZ. Cryst Struct Comm 1981;10:489.

954. Katritzky AR, Savage GP, Palenik GJ, Qian K, Zhang Z, Durst HD. J Chem Soc, Perkin Trans 2 1990:1657.

955. Boeckman RK, Shao P, Mullins JJ. Org Synth 2000;77:141.

956. Frigerio M, Santagostino M, Sputore S. J Org Chem 1999;64:4537.

957. Gallen MJ, Goumont R, Clark T, Terrier F, Williams CM. Angew Chem, Int Ed 2006;45:2929.

958. Waters T, Boulton J, Clark T, Gallen MJ, Williams CM, O’Hair RAJ. Org Biomol Chem 2008;6:2530. [PubMed: 18600274]

959. Ozanne A, Pouysegu L, Depernet D, Francois B, Quideau S. Org Lett 2003;5:2903. [PubMed: 12889904] 
960. Lebrasseur N, Gagnepain J, Ozanne-Beaudenon A, Leger J-M, Quideau S. J Org Chem 2007;72:6280. [PubMed: 17628111]

961. Gagnepain J, Castet F, Quideau S. Ang Chem Int Ed 2007;46:1533.

962. Ozanne-Beaudenon A, Quideau S. Tetrahedron Lett 2006;47:5869.

963. Thottumkara AP, Vinod TK. Tetrahedron Lett 2002;43:569.

964. Kommreddy A, Bowsher MS, Gunna MR, Botha K, Vinod TK. Tetrahedron Lett 2008;49:4378. 965. Stickley SH, Martin JC. Tetrahedron Lett 1995;36:9117.

966. Richardson RD, Zayed JM, Altermann S, Smith D, Wirth T. Angew Chem, Int Ed 2007;46:6529.

967. Moorthy JN, Singhal N, Senapati K. Tetrahedron Lett 2008;49:80.

968. Kano N, Ohashi M, Hoshiba K, Kawashima T. Tetrahedron Lett 2004;45:8173.

969. Muelbaier M, Giannis A. Angew Chem, Int Ed 2001;40:4393.

970. Sorg G, Mengei A, Jung G, Rademann J. Angew Chem, Int Ed 2001;40:4395.

971. Lei ZQ, Ma HC, Zhang Z, Yang YX. React Funct Polym 2006;66:840.

972. Lei Z, Denecker C, Jegasothy S, Sherrington DC, Slater NKH, Sutherland AJ. Tetrahedron Lett 2003;44:1635.

973. Bromberg L, Zhang H, Hatton TA. Chem Mater 2008;20:2001.

974. Frigerio M, Santagostino M. Tetrahedron Lett 1994;35:8019.

975. Paintner FF, Allmendinger L, Bauschke G. Synthesis 2001:2113.

976. Martin C, Macintosh N, Lamb N, Fallis AG. Org Lett 2001;3:1021. [PubMed: 11277785]

977. Kirsch S, Bach T. Angew Chem, Int Ed 2003;42:4685.

978. Iwamoto O, Koshino H, Hashizume D, Nagasawa K. Angew Chem, Int Ed 2007;46:8625.

979. Nicolaou KC, Harrison ST. Angew Chem, Int Ed 2006;45:3256.

980. Venkatesan K, Srinivasan KV. Tetrahedron: Asymmetry 2008;19:209.

981. Skouta R, Li C-J. Tetrahedron Lett 2007;48:8343.

982. Kuboki A, Yamamoto T, Taira M, Arishige T, Ohira S. Tetrahedron Lett 2007;48:771.

983. Hosokawa S, Kuroda S, Imamura K, Tatsuta K. Tetrahedron Lett 2006;47:6183.

984. Ichikawa Y, Yamaoka T, Nakano K, Kotsuki H. Org Lett 2007;9:2989. [PubMed: 17602639]

985. Zhang J, Wang X, Wang W, Quan W, She X, Pan X. Tetrahedron 2007;63:6990.

986. Vincent A, Prunet J. Synlett 2006:2269.

987. Suzuki K, Takayama H. Org Lett 2006;8:4605. [PubMed: 16986961]

988. Mohapatra DK, Mondal D, Gonnade RG, Chorghade MS, Gurjar MK. Tetrahedron Lett 2006;47:6031.

989. Kirkham JED, Lee V, Baldwin JE. Chem Commun 2006:2863.

990. Kaluza Z, Mostowicz D, Dolega G, Wojcik R. Tetrahedron 2008;64:2321.

991. Chen JJ, Aduda V. Synth Commun 2007;37:3493.

992. Molander GA, Petrillo DE. J Am Chem Soc 2006;128:9634. [PubMed: 16866509]

993. More JD, Finney NS. Org Lett 2002;4:3001. [PubMed: 12182609]

994. More JD, Finney NS. Synlett 2003:1307.

995. Liu Z, Chen Z-C, Zheng Q-G. Org Lett 2003;5:3321. [PubMed: 12943417]

996. Kuhakarn C, Kittigowittana K, Ghabkham P, Pohmakotr M, Reutrakul V. Synth Commun 2006;36:2887.

997. Moorthy JN, Singhal N, Senapati K. Org Biomol Chem 2007;5:767. [PubMed: 17315062]

998. Corey EJ, Palani A. Tetrahedron Lett 1995;36:3485.

999. Corey EJ, Palani A. Tetrahedron Lett 1995;36:7945.

1000. De Munari S, Frigerio M, Santagostino M. J Org Chem 1996;61:9272.

1001. Schulze A, Giannis A. Adv Synth Catal 2004;346:252.

1002. Schulze A, Giannis A. Synthesis 2006:257.

1003. Thottumkara AP, Bowsher MS, Vinod TK. Org Lett 2005;7:2933. [PubMed: 15987173]

1004. Page PCB, Appleby LF, Buckley BR, Allin SM, McKenzie MJ. Synlett 2007:1565. 
1005. Magdziak D, Rodriguez AA, Van De Water RW, Pettus TRR. Org Lett 2002;4:285. [PubMed: 11796071]

1006. Kuboki A, Yamamoto T, Ohira S. Chem Lett 2003;32:420.

1007. Huang Y, Zhang J, Pettus TRR. Org Lett 2005;7:5841. [PubMed: 16354080]

1008. Nicolaou KC, Montagnon T, Baran PS, Zhong YL. J Am Chem Soc 2002;124:2245. [PubMed: 11878978]

1009. Nicolaou KC, Baran PS, Zhong Y-L. J Am Chem Soc 2001;123:3183. [PubMed: 11457049]

1010. Moorthy JN, Singhal N, Senapati K. Tetrahedron Lett 2006;47:1757.

1011. Nicolaou KC, Mathison CJN, Montagnon T. J Am Chem Soc 2004;126:5192. [PubMed: 15099102]

1012. Ma HC, Jiang XZ. Synthesis 2007:412.

1013. Chaudhari KH, Mahajan US, Bhalerao DS, Akamanchi KG. Synlett 2007:2815.

1014. Bhalerao DS, Mahajan US, Chaudhari KH, Akamanchi KG. J Org Chem 2007;72:662. [PubMed: 17221993]

1015. Arote ND, Bhalerao DS, Akamanchi KG. Tetrahedron Lett 2007;48:3651.

1016. Chiampanichayakul S, Pohmakotr M, Reutrakul V, Jaipetch T, Kuhakarn C. Synthesis 2008:2045.

1017. Anwar HF, Hansen TV. Tetrahedron Lett 2008;49:4443.

1018. Reddy MS, Narender M, Mahesh A, Nageswar YVD, Rao KR. Synth Commun 2006;36:3771.

1019. Yadav JS, Reddy BVS, Reddy CS, Krishna AD. Synthesis 2007:693.

1020. Yadav JS, Reddy BVS, Reddy CS, Krishna AD. Tetrahedron Lett 2007;48:2029.

1021. Yadav JS, Reddy BVS, Basak AK, Baishya G, Narsaiah AV. Synthesis 2006:451.

1022. Kirsch SF. J Org Chem 2005;70:10210. [PubMed: 16292876]

1023. Crone B, Kirsch SF. Chem Commun 2006:764.

1024. Martinez-Bescos P, Cagide-Fagin F, Roa LF, Ortiz-Lara JC, Kierus K, Ozores-Viturro L, Fernandez-Gonzalez M, Alonso R. J Org Chem 2008;73:3745. [PubMed: 18429636]

1025. Heravi MM, Bakhtiari K, Tehrani MH, Javadi NM, Oskooie HA. ARKIVOC 2006;xvi:16.

1026. Nicolaou KC, Baran PS, Zhong YL, Barluenga S, Hunt KW, Kranich R, Vega JA. J Am Chem Soc 2002;124:2233. [PubMed: 11878977]

1027. Janza B, Studer A. J Org Chem 2005;70:6991. [PubMed: 16095334]

1028. Das B, Holla H, Mahender G, Banerjee J, Reddy MR. Tetrahedron Lett 2004;45:7347.

1029. Yadav JS, Reddy BVS, Singh AP, Basak AK. Tetrahedron Lett 2007;48:4169.

1030. Ngouansavanh T, Zhu J. Angew Chem, Int Ed 2006;45:3495.

1031. Ngouansavanh T, Zhu J. Angew Chem, Int Ed 2007;46:5775.

1032. Fontaine P, Chiaroni A, Masson G, Zhu J. Org Lett 2008;10:1509. [PubMed: 18345680]

1033. Nicolaou KC, Zhong Y-L, Baran PS. Angew Chem, Int Ed 2000;39:622.

1034. Nicolaou KC, Baran PS, Kranich R, Zhong Y-L, Sugita K, Zou N. Angew Chem, Int Ed 2001;40:202.

1035. Nicolaou KC, Baran PS, Zhong Y-L, Vega JA. Angew Chem, Int Ed 2000;39:2525.

1036. Dess DB, Martin JC. J Org Chem 1983;48:4155.

1037. Ireland RE, Liu L. J Org Chem 1993;58:2899.

1038. Myers AG, Zhong B, Movassaghi M, Kung DW, Lanman BA, Kwon S. Tetrahedron Lett 2000;41:1359.

1039. Meyer SD, Schreiber SL. J Org Chem 1994;59:7549.

1040. Deng G, Xu B, Liu C. Tetrahedron 2005;61:5818.

1041. Candela Lena JI, Martin Hernando JI, Rico Ferreira MdR, Altinel E, Arseniyadis S. Synlett 2001:597.

1042. Thongsornkleeb C, Danheiser RL. J Org Chem 2005;70:2364. [PubMed: 15760233]

1043. Li P, Majireck MM, Korboukh I, Weinreb SM. Tetrahedron Lett 2008;49:3162. [PubMed: 19421308]

1044. Sharma PK, Kolchinski A, Shea HA, Nair JJ, Gou Y, Romanczyk LJ Jr, Schmitz HH. Org Process Res Dev 2007;11:422. 
1045. Trost BM, O’Boyle BM. Org Lett 2008;10:1369. [PubMed: 18318539]

1046. Nicolaou KC, Tang Y, Wang J. Chem Commun 2007:1922.

1047. England DB, Padwa A. J Org Chem 2008;73:2792. [PubMed: 18318547]

1048. Zhang W, Wicks MN, Burn PL. Org Biomol Chem 2008;6:879. [PubMed: 18292879]

1049. Struble JR, Kaeobamrung J, Bode JW. Org Lett 2008;10:957. [PubMed: 18266380]

1050. Nourry A, Legoupy S, Huet F. Tetrahedron 2008;64:2241.

1051. Schweizer E, Gaich T, Brecker L, Mulzer J. Synthesis 2007:3807.

1052. Migita A, Shichijo Y, Oguri H, Watanabe M, Tokiwano T, Oikawa H. Tetrahedron Lett 2008;49:1021.

1053. Rein C, Demel P, Outten RA, Netscher T, Breit B. Angew Chem, Int Ed 2007;46:8670.

1054. Kozaka T, Miyakoshi N, Mukai C. J Org Chem 2007;72:10147. [PubMed: 18044932]

1055. Pouplin T, Tolon B, Nuhant P, Delpech B, Marazano C. Eur J Org Chem 2007:5117.

1056. Trost BM, Waser J, Meyer A. J Am Chem Soc 2007;129:14556. [PubMed: 17985906]

1057. Veitch GE, Beckmann E, Burke BJ, Boyer A, Ayats C, Ley SV. Angew Chem, Int Ed 2007;46:7633.

1058. Huang J, Wu C, Wulff WD. J Am Chem Soc 2007;129:13366. [PubMed: 17929921]

1059. Kotoku N, Narumi F, Kato T, Yamaguchi M, Kobayashi M. Tetrahedron Lett 2007;48:7147.

1060. Krishna PR, Sreeshailam A. Tetrahedron Lett 2007;48:6924.

1061. Scheerer JR, Lawrence JF, Wang GC, Evans DA. J Am Chem Soc 2007;129:8968. [PubMed: 17602636]

1062. Jung W-H, Harrison C, Shin Y, Fournier J-H, Balachandran R, Raccor BS, Sikorski RP, Vogt A, Curran DP, Day BW. J Med Chem 2007;50:2951. [PubMed: 17542572]

1063. Ahmad NM, Rodeschini V, Simpkins NS, Ward SE, Blake AJ. J Org Chem 2007;72:4803. [PubMed: 17530804]

1064. Wang H, Shuhler BJ, Xian M. J Org Chem 2007;72:4280. [PubMed: 17447817]

1065. Liu Q, Rovis T. Org Process Res Dev 2007;11:598. [PubMed: 19603085]

1066. Neumann J, Weingarten S, Thiem J. Eur J Org Chem 2007:1130.

1067. Takayama H, Kato K, Kimura M, Akita H. Heterocycles 2007;71:75.

1068. Bjerre J, Fenger TH, Marinescu LG, Bols M. Eur J Org Chem 2007:704.

1069. Chen J, Chen X, Willot M, Zhu J. Angew Chem, Int Ed 2006;45:8028.

1070. Watanabe H, Takano M, Umino A, Ito T, Ishikawa H, Nakada M. Org Lett 2007;9:359. [PubMed: 17217304]

1071. de Vicente J, Huckins JR, Rychnovsky SD. Angew Chem, Int Ed 2006;45:7258.

1072. Shah U, Chackalamannil S, Ganguly AK, Chelliah M, Kolotuchin S, Buevich A, McPhail A. J Am Chem Soc 2006;128:12654. [PubMed: 17002352]

1073. Geng Z, Chen B, Chiu P. Angew Chem, Int Ed 2006;45:6197.

1074. Garg NK, Hiebert S, Overman LE. Angew Chem, Int Ed 2006;45:2912.

1075. Nicolaou KC, Zhong YL, Baran PS, Jung J, Choi HS, Yoon WH. J Am Chem Soc 2002;124:2202. [PubMed: 11878974]

1076. Nicolaou KC, Jung J, Yoon WH, Fong KC, Choi HS, He Y, Zhong YL, Baran PS. J Am Chem Soc 2002; 124:2183. [PubMed: 11878972]

1077. Nicolaou KC, Baran PS, Zhong YL, Fong KC, Choi HS. J Am Chem Soc 2002;124:2190. [PubMed: 11878973]

1078. Nicolaou KC, Baran PS, Zhong YL, Sugita K. J Am Chem Soc 2002;124:2212. [PubMed: 11878975]

1079. Karade NN, Gampawar SV, Kondre JM, Shinde SV. ARKIVOC 2008;xii:9.

1080. Yadav JS, Reddy BVS, Singh AP, Basak AK. Synthesis 2008:469.

1081. Yadav JS, Reddy BVS, Mandal SS, Basak AK, Madavi C, Kunwar AC. Synlett 2008:1175.

1082. Ma HC, Jiang XZ. Synlett 2007:1679.

1083. Salgaonkar PD, Shukla VG, Akamanchi KG. Synth Commun 2007;37:275.

1084. Telvekar VN, Arote ND, Herlekar OP. Synlett 2005:2495. 
1085. Mahajan US, Akamanchi KG. Synlett 2008:987.

1086. Karade NN, Tiwari GB, Shinde SV, Gampawar SV, Kondre JM. Tetrahedron Lett 2008;49:3441.

1087. Bandgar SB, Bandgar BP, Korbad BL, Sawant SS. Tetrahedron Lett 2007;48:1287.

1088. Nicolaou KC, Mathison CJN. Angew Chem, Int Ed 2005;44:5992.

\section{Biographies}

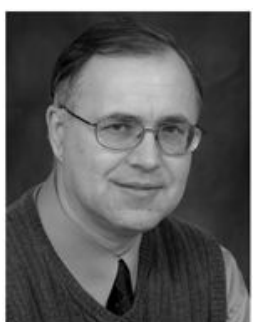

Viktor V. Zhdankin was born in Ekaterinburg, Russian Federation. His M.S. (1978), Ph.D. (1981), and Doctor of Chemical Sciences (1986) degrees were earned at Moscow State University in the research laboratories of Professor Nikolay S. Zefirov. He moved to the University of Utah in 1990, where he worked for three years as Instructor of organic chemistry and Research Associate. In 1993, he joined the faculty of the University of Minnesota Duluth, where he is currently a Professor of Chemistry. He has published over 200 scientific papers including 21 reviews and book chapters. His main research interests are in the fields of synthetic and mechanistic organic chemistry of hypervalent main-group elements (iodine, xenon, selenium, sulfur, and phosphorus) and organofluorine chemistry.

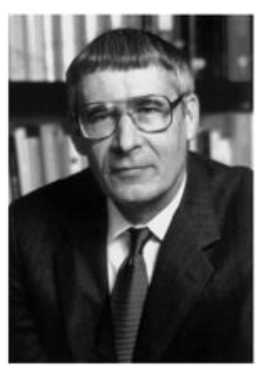

Peter J. Stang is a Distinguished Professor of Chemistry at Utah where he has been since 1969. He is a member of the US National Academy of Sciences and a Fellow of the American Academy of Arts and Sciences as well as a foreign member of the Chinese Academy of Sciences and the Hungarian Academy of Sciences. His current research interest is self-assembly and supramolecular chemistry. He is the author or co-author of 430 scientific publications including seven monographs and two dozen review articles. Since January 2002 he is the Editor of the Journal of the American Chemical Society (JACS). 


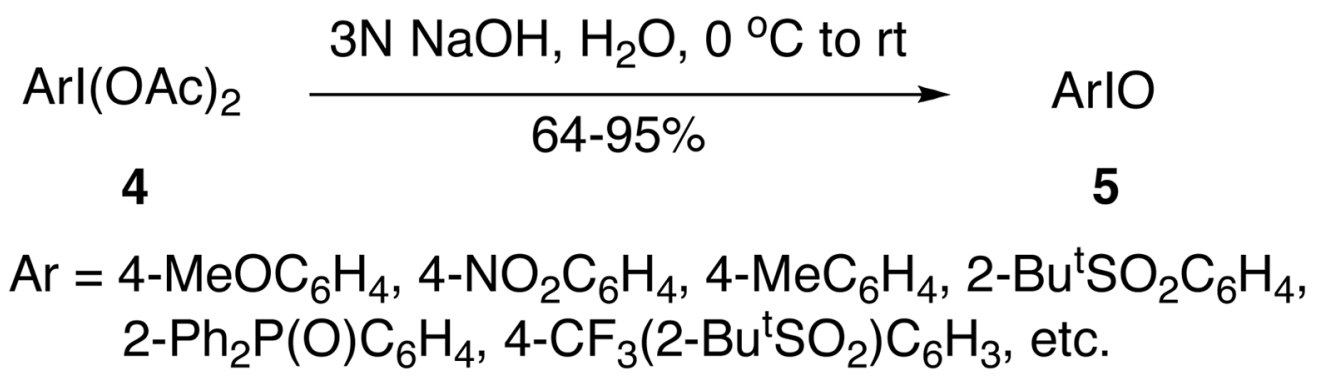

Scheme 1. 


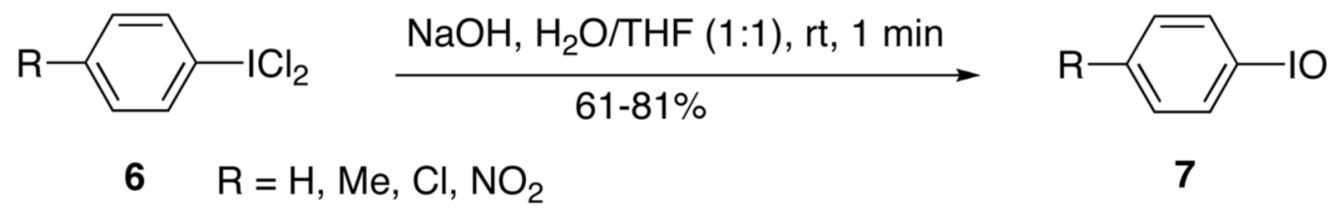

Scheme 2. 


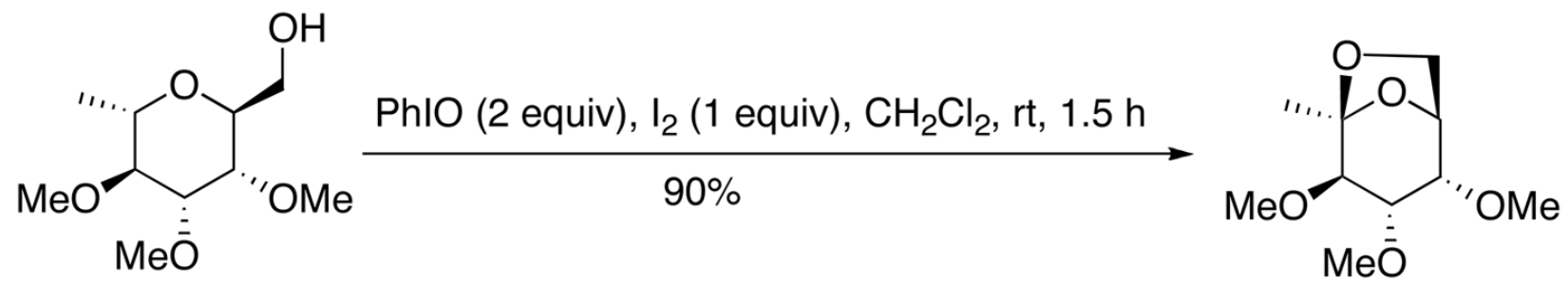

17

$(\mathrm{PhO})_{2}(\mathrm{O}) \mathrm{P}$

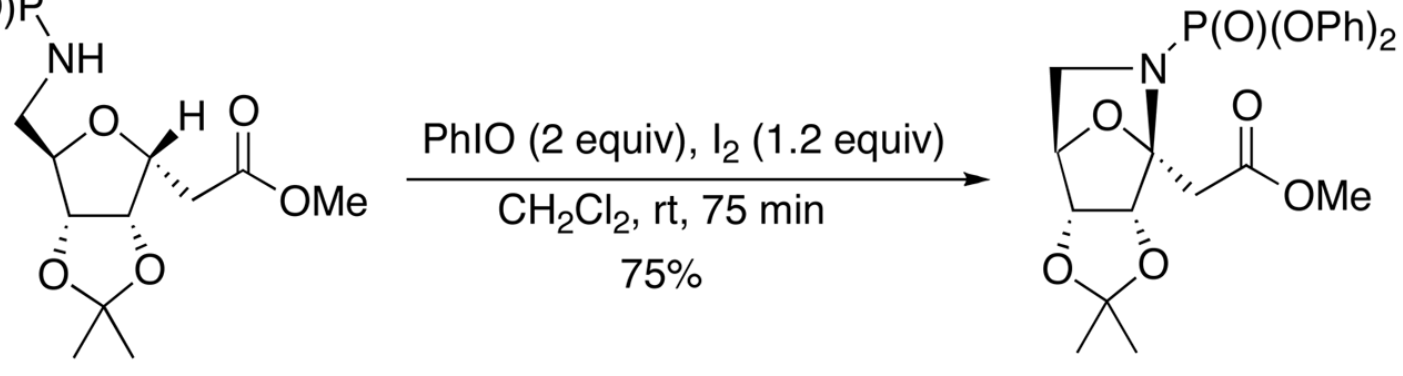

18

Scheme 3. 


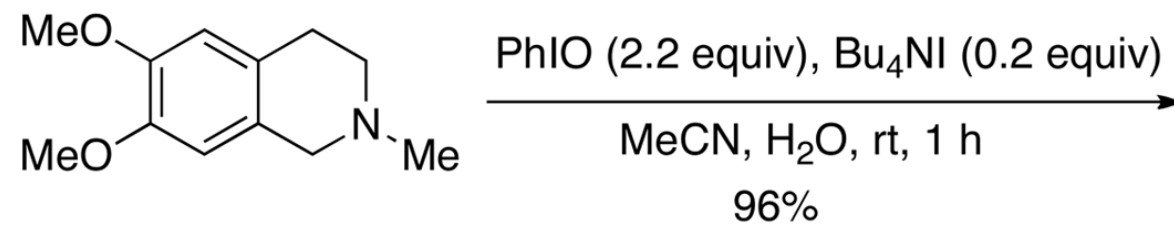

19<smiles>COc1cc2c(cc1OC)C(=O)N(C)CC2</smiles>

20

Scheme 4. 
$\mathrm{HO}$<smiles>CC1CCCNC1</smiles>

21

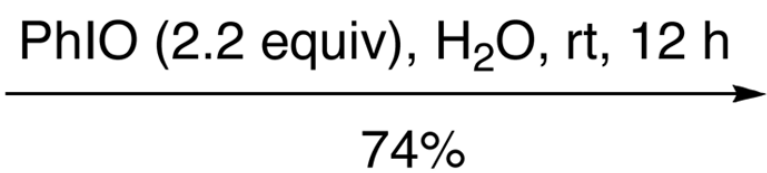

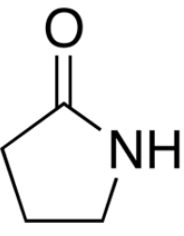

22

Scheme 5 . 
<smiles>[R]CC([R7])O</smiles>

23

$$
\begin{aligned}
& \text { PhIO (3 equiv), } \mathrm{TsOH} \cdot \mathrm{H}_{2} \mathrm{O} \text { (2.5 equiv) } \\
& \mathrm{MeCN}, \Delta, 1.5 \mathrm{~h} \\
& 80-96 \%
\end{aligned}
$$<smiles>[R]C(=O)C([R])[O-]</smiles>

24

$\mathrm{R}^{1}=\mathrm{H}, \mathrm{Ph}, 4-\mathrm{MeC}_{6} \mathrm{H}_{4}, 4-\mathrm{ClC}_{6} \mathrm{H}_{4}, 4-\mathrm{NO}_{2} \mathrm{C}_{6} \mathrm{H}_{4}, \mathrm{C}_{5} \mathrm{H}_{11}$, etc. $\mathrm{R}^{2}=\mathrm{H}, \mathrm{Me}, \mathrm{C}_{7} \mathrm{H}_{15}, \mathrm{C}_{4} \mathrm{H}_{9}$, etc.

1. $\mathrm{PhlO}$ (3 equiv), $\mathrm{TsOH} \cdot \mathrm{H}_{2} \mathrm{O}$ (2.5 equiv), $\mathrm{MeCN}$

2.<smiles>[R]C(N)=[V]C(=O)OC(C)(C)C</smiles><smiles>[R]c1[X]c([R])c([R])[nH]1</smiles>

$\mathrm{R}^{1}=\mathrm{Ph}, 4-\mathrm{MeC}_{6} \mathrm{H}_{4}, 4-\mathrm{ClC}_{6} \mathrm{H}_{4}, 4-\mathrm{NO}_{2} \mathrm{C}_{6} \mathrm{H}_{4}$ $\mathrm{R}^{2}=\mathrm{H} ; \mathrm{R}^{3}=\mathrm{Me}, \mathrm{Ph} ; \mathrm{X}=\mathrm{S}, \mathrm{NH}$

1. $\mathrm{PhIO}$ (3 equiv), $\mathrm{TsOH} \bullet \mathrm{H}_{2} \mathrm{O}$ (2.5 equiv), $\mathrm{MeCN}$

2.<smiles>CC(=O)C(C)(C)C(=O)Nc1ccccc1</smiles><smiles>[R]c1cc2ccccn2c1[R6]</smiles>

$\mathrm{R}^{1}=\mathrm{Ph}, 4-\mathrm{ClC}_{6} \mathrm{H}_{4} ; \mathrm{R}^{2}=\mathrm{H}$

Scheme 6. 

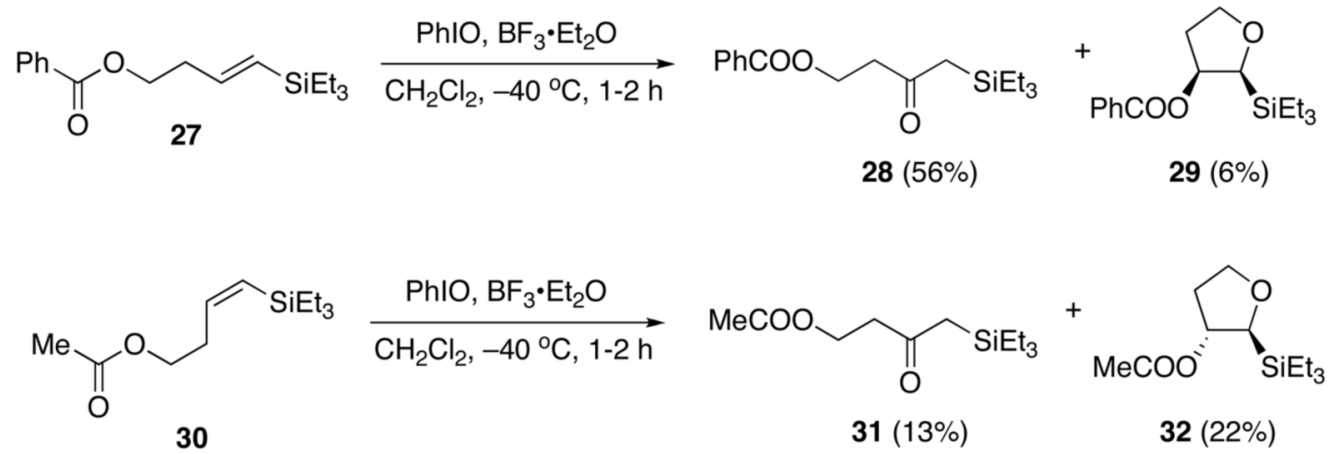

Scheme 7. 


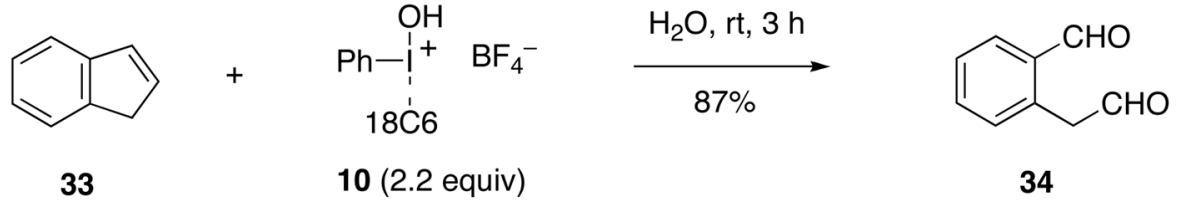

Scheme 8. 


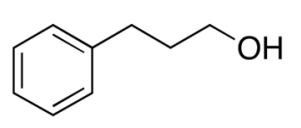

35

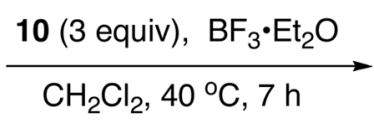

$$
\begin{aligned}
& 63 \%
\end{aligned}
$$

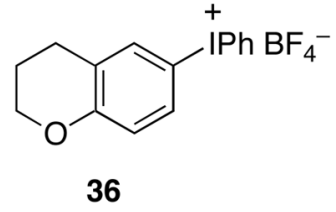

Scheme 9. 

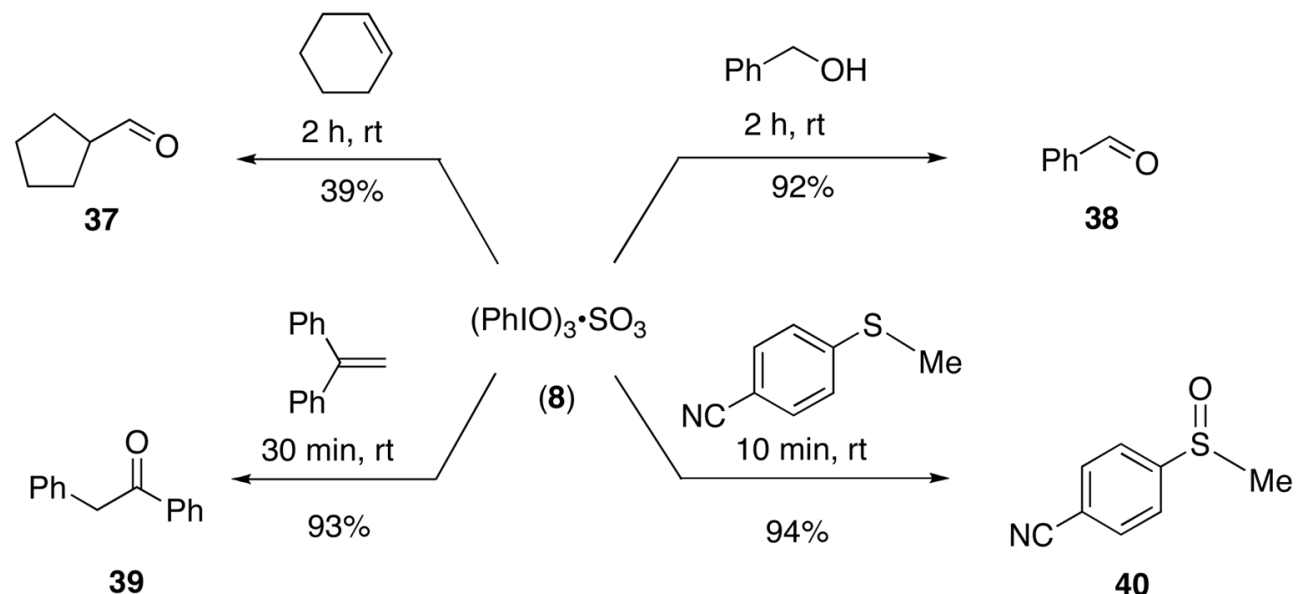

Scheme 10. 
<smiles>[R]C1=CCCCC1=O</smiles>

$\mathrm{PhIO}, \mathrm{CDCl}_{3}, \mathrm{rt}, 2-18 \mathrm{~h}$

$45-91 \%$<smiles>[R]C12OC1CCCC2=O</smiles>

42

Scheme 11. 


$$
\begin{array}{ccc}
\mathrm{Arl}+\mathrm{XeF}_{2} \stackrel{\mathrm{CH}_{2} \mathrm{Cl}_{2}, \mathrm{HF} \text { (anhyd), rt, 1-3 h }}{-\mathrm{Xe}} \underset{\mathrm{ArIF}}{2} & \begin{array}{c}
43 \\
\mathrm{Ar}=\mathrm{Ph}, 3-\mathrm{ClC}_{6} \mathrm{H}_{4}, 3-\mathrm{NO}_{2} \mathrm{C}_{6} \mathrm{H}_{4}, 4-\mathrm{MeOC}_{6} \mathrm{H}_{4}, 3-\mathrm{MeOC}_{6} \mathrm{H}_{4}
\end{array}
\end{array}
$$

Scheme 12. 

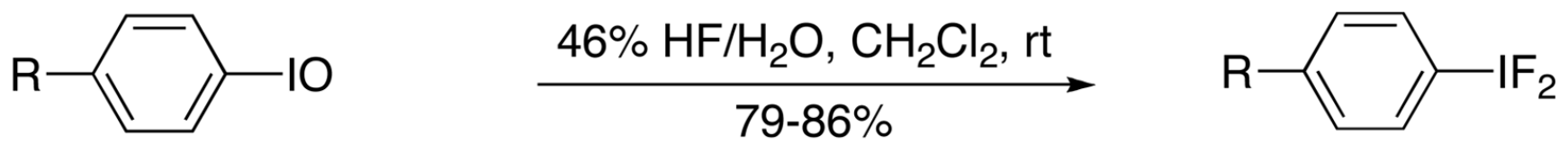

$44 \mathrm{R}=\mathrm{H}, \mathrm{Me}, \mathrm{Cl}, \mathrm{NO}_{2}$

45

Scheme 13. 
<smiles>[R]C(=O)CC([R])=O</smiles>

46

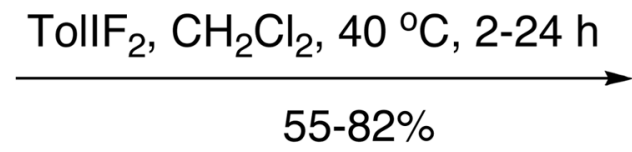

$\mathrm{R}^{1}=\mathrm{Me}, \mathrm{Pr}, \mathrm{Ph}$, etc. $\mathrm{R}^{2}=\mathrm{OC}_{5} \mathrm{H}_{11}, \mathrm{Ph}, \mathrm{Et}, \mathrm{Bu}^{\mathrm{t}}, \mathrm{NMe}_{2}, \mathrm{NPr}_{2}^{\mathrm{i}}$, etc.<smiles>[R]C(=O)C(F)C([R])=O</smiles>

47

Scheme 14. 
<smiles>O=C(CSc1ccccc1)O[Na]</smiles>

48
TollF ${ }_{2}$ (1 equiv), $\mathrm{CH}_{2} \mathrm{Cl}_{2}, 0^{\circ} \mathrm{C}, 12 \mathrm{~h}$ $64-72 \%$

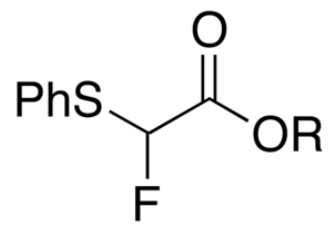

49

Scheme 15. 


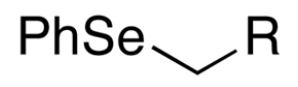

50

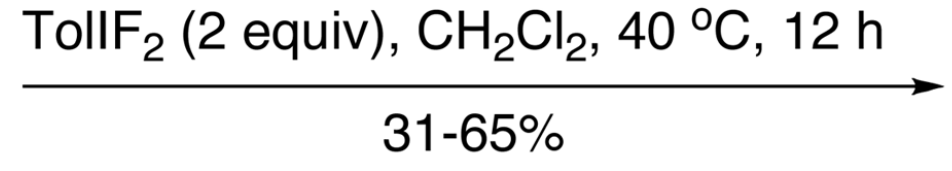

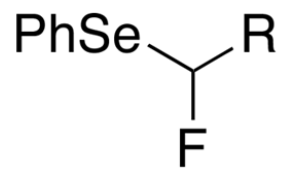

51

$\mathrm{R}=\mathrm{CO}_{2} \mathrm{Et}, \mathrm{CO}_{2} \mathrm{Ph}, \mathrm{CO}_{2} \mathrm{CH}_{2} \mathrm{CH}=\mathrm{CH}_{2}, \mathrm{CONHMe}, \mathrm{CONMe}_{2}$, CN, etc. 
<smiles>[R][C@]1(CI)C[C@@H]([15CH2])O1</smiles>

52 $\mathrm{R}=\mathrm{H}$ or $\mathrm{Me}$ $\underset{66-70 \%}{\stackrel{\text { TollF}_{2}\left(1.3 \text { equiv), } \mathrm{Et}_{3} \mathrm{~N} \cdot 5 \mathrm{HF}, \mathrm{CH}_{2} \mathrm{Cl}_{2}, \mathrm{rt}, 1 \mathrm{~h}\right.}{\longrightarrow}}$ $6-70 \%$

$53 \quad \mathrm{R}^{1}=\mathrm{C}_{5} \mathrm{H}_{11}, \mathrm{Ph}$ and $\mathrm{R}^{2}=\mathrm{H}$ or $\mathrm{R}^{1}=\mathrm{H}, \mathrm{R}^{2}=\mathrm{Ph}$<smiles>CCCC1CCCC(C)(CI)O1</smiles>

$\underset{50 \%}{\stackrel{\text { TollF }_{2}\left(1.3 \text { equiv), } \mathrm{Et}_{3} \mathrm{~N} \cdot 5 \mathrm{HF}, \mathrm{CH}_{2} \mathrm{Cl}_{2}, \mathrm{rt}, 1 \mathrm{~h}\right.}{\longrightarrow}}$

54<smiles>CCCC1CCCC(C)(F)CO1</smiles>

57

Scheme 17. 
<smiles>[R7]C([R7])=NN[AsH3-]</smiles>

$\mathrm{PhIF}_{2}$, 2-chloropyridine

58<smiles>[R]C([R7])=[N+]=[N-]</smiles><smiles>[R]C(=O)O</smiles>

59<smiles>[R]C(=O)OC([R])[R]</smiles>

$60(53-92 \%)$

$\mathrm{R}^{1}, \mathrm{R}^{2}=\mathrm{H}$, alkyl, aryl; $\mathrm{R}^{3}=$ alkyl, aryl, etc.

Scheme 18. 


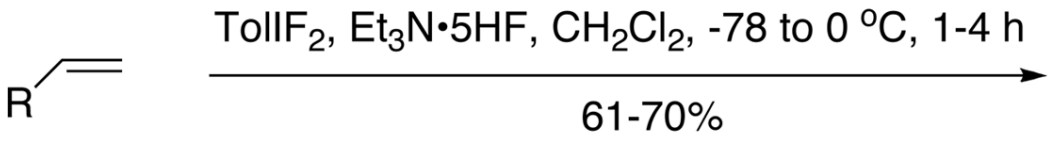

61<smiles>[R]C(F)CF</smiles>

62

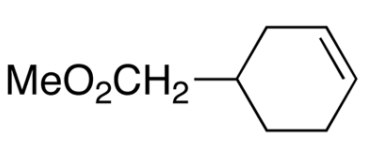

63<smiles>[R]C1CC=C(C)CC1</smiles>

65
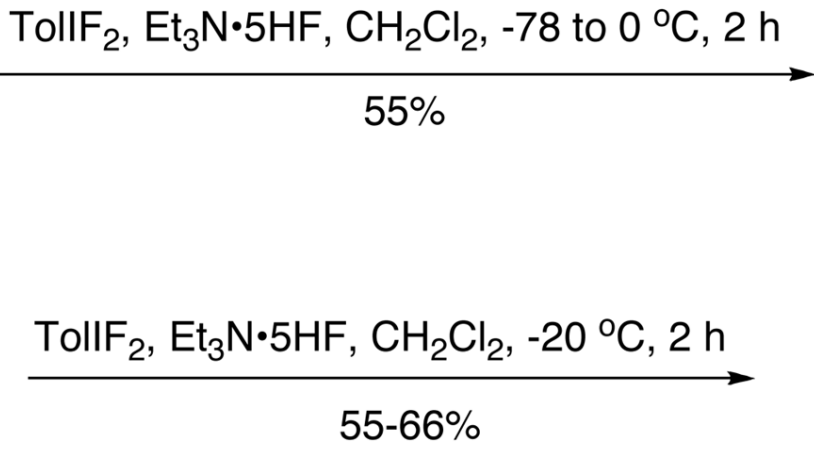

$$
\mathrm{R}=\mathrm{CO}_{2} \mathrm{Me}, \mathrm{C}(\mathrm{O}) \mathrm{Me}, \mathrm{Bu}^{\mathrm{t}}
$$

Scheme 19.

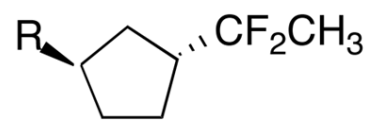

66 


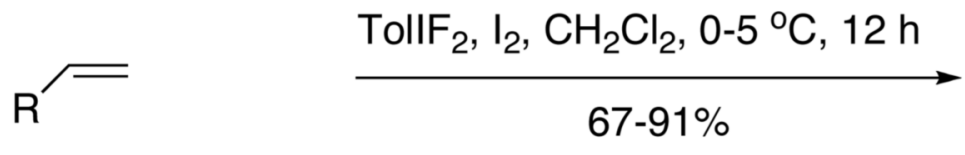

67 $\mathrm{R}=n-\mathrm{C}_{6} \mathrm{H}_{13}, \mathrm{Ph}, 4-\mathrm{Bu}^{\mathrm{t}} \mathrm{C}_{6} \mathrm{H}_{4}, \mathrm{PhCH}_{2}$, etc.

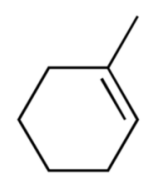

69
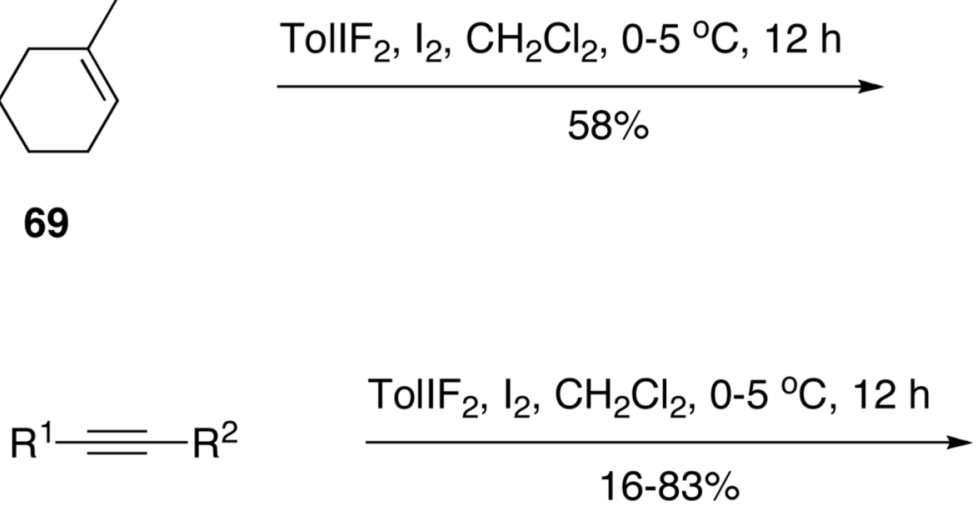

71

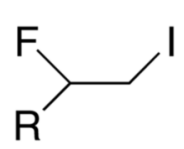

68

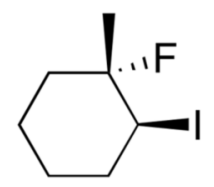

70

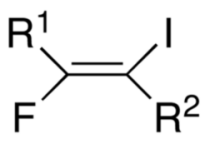

72

$$
R^{1}=R^{2}=\operatorname{Pr} ; R^{1}=R^{2}=P h ; R^{1}=P h, R^{2}=M e ; R^{1}=P h, R^{2}=H
$$

Scheme 20. 


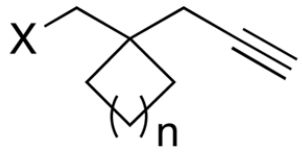

73
$\mathrm{TollF}_{2}, \mathrm{BF}_{3} \cdot \mathrm{Pr}_{2}{ }_{2} \mathrm{O},-60^{\circ} \mathrm{C}$ to rt

$84-92 \%$

$\mathrm{X}=\mathrm{Cl}, \mathrm{Br} ; \mathrm{n}=1-3$

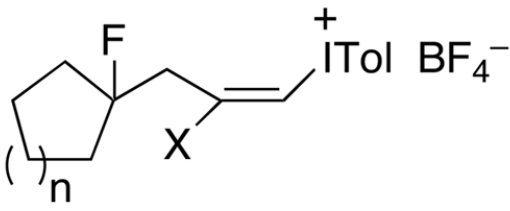

74

Scheme 21. 

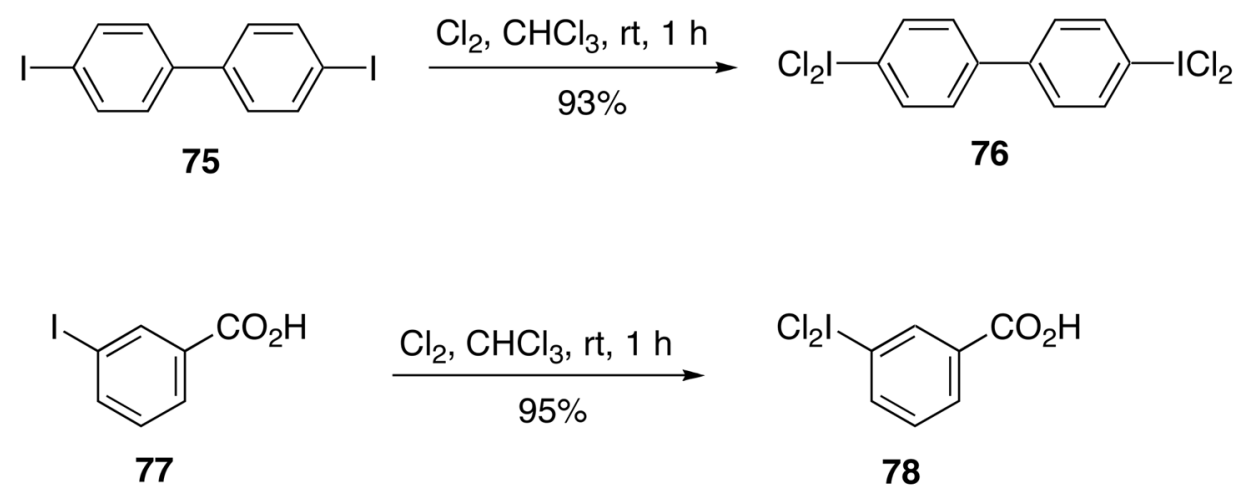

Scheme 22. 
$\mathrm{NaClO}(5.84 \%), \mathrm{HCl}, \mathrm{H}_{2} \mathrm{O}, 15^{\circ} \mathrm{C}, 5 \mathrm{~min}$

Arl

79 $94-100 \%$

$\mathrm{ArICl}_{2}$

80

\section{$\mathrm{Ar}=\mathrm{Ph}, 4-\mathrm{MeC}_{6} \mathrm{H}_{4}, 2-\mathrm{FC}_{6} \mathrm{H}_{4}, 2-\mathrm{BrC}_{6} \mathrm{H}_{4}, 3-\mathrm{BrC}_{6} \mathrm{H}_{4}, 4-\mathrm{BrC}_{6} \mathrm{H}_{4}$, $4-\mathrm{ClC}_{6} \mathrm{H}_{4}, 3-\mathrm{NO}_{2} \mathrm{C}_{6} \mathrm{H}_{4}, 4-\mathrm{NO}_{2} \mathrm{C}_{6} \mathrm{H}_{4}, 4-\mathrm{PhC}_{6} \mathrm{H}_{4}$}

Scheme 23. 


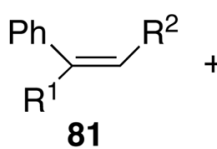

81

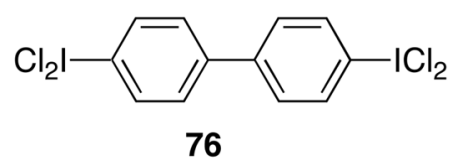

76

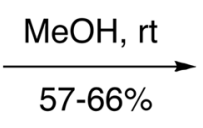

$R^{1}=H, R^{2}=H ; R^{1}=P h, R^{2}=H ; R^{1}=H, R^{2}=P h$

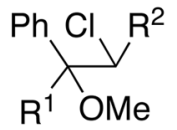

82

Scheme 24. 
$\mathrm{PhICl}_{2}$, THF, pyridine, 0 to $3^{\circ} \mathrm{C}$<smiles>CC(=O)c1ccc(N)cc1</smiles>

83

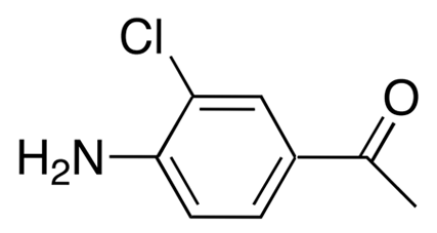

84

Scheme 25. 
<smiles>CC(=O)C(C)C(=O)O</smiles>

85

$\mathrm{R}=\mathrm{Et}, \mathrm{Bn}, \mathrm{Ph}$, or

9-(anthracenyl) $\mathrm{CH}_{2}$
$\mathrm{TollCl}_{2}, 86$ (5 mol\%),

pyridine $\left(1.2\right.$ equiv), toluene, $50^{\circ} \mathrm{C}, 20 \mathrm{~h}$

$37-82 \%$

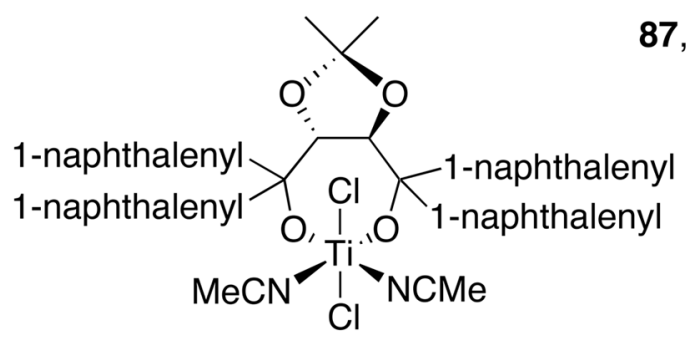

86

Scheme 26. 
<smiles>CC(C)OC(=O)N1CCCC1</smiles>

4- $\mathrm{NO}_{2} \mathrm{C}_{6} \mathrm{H}_{4} \mathrm{ICl}_{2}$ (4 equiv), $\mathrm{MeCN} / \mathrm{H}_{2} \mathrm{O}, 45^{\circ} \mathrm{C}$ $88 \%$

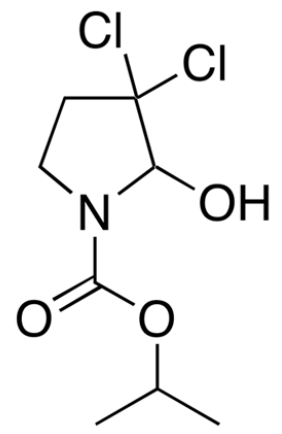

89

Scheme 27. 


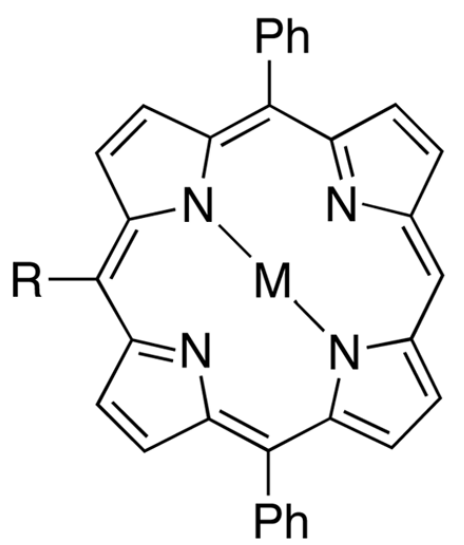<smiles>O=S(=O)(O[Na])C(c1ccccc1)c1ccccc1</smiles>

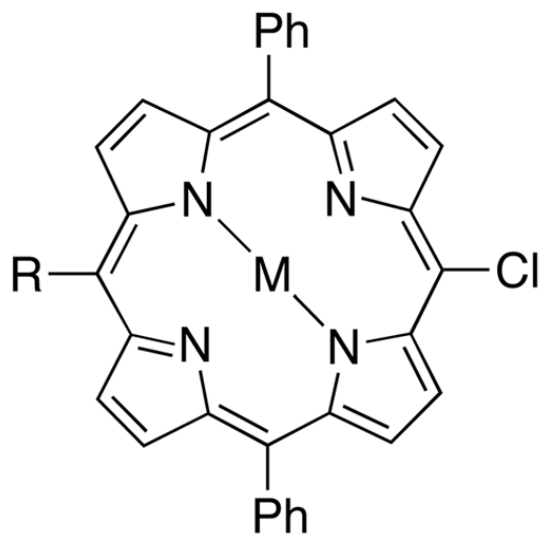

90

$\mathrm{M}=2 \mathrm{H}, \mathrm{Cu}, \mathrm{Ni}$

$\mathrm{R}=\mathrm{Ph}$ or $4-\mathrm{CF}_{3} \mathrm{C}_{6} \mathrm{H}_{4}$

Scheme 28. 

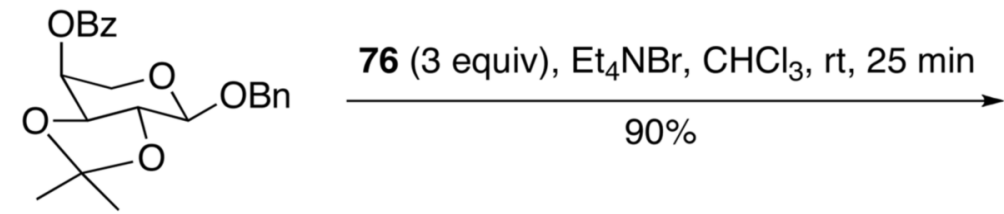

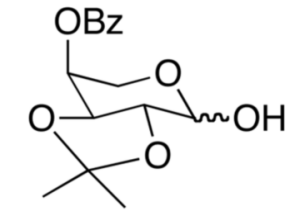

\section{2}

Scheme 29. 
<smiles>CCc1c(O)cc(=O)[nH]c1C</smiles>

93<smiles>[R]c1cc([R])cc(Cl)c1</smiles>

94

$$
R^{1}=R^{2}=H ; R^{1}=H, R^{2}=M e ; R^{1}=M e, R^{2}=M e
$$<smiles>[R2]c1cc([R])cc([I+]c2c([O-])c(CC)c(C)[nH]c2=O)c1</smiles>

DMF, reflux<smiles>[R7]c1ccc(Oc2c(CC)c(C)[nH]c(=O)c2I)c([R])c1</smiles>

$95(88-93 \%)$

Scheme 30. 
<smiles>[R]c1cccc([X])c1[R7]</smiles>

96 $\underset{69-86 \%}{\stackrel{\mathrm{PhICl}_{2}, \mathrm{KSCN}, \mathrm{CH}_{2} \mathrm{Cl}_{2}, 0^{\circ} \mathrm{C} \text { to rt, } 50 \mathrm{~min}}{\longrightarrow}}$

$\mathrm{X}=\mathrm{OH}$ or $\mathrm{NH}_{2}$

$\mathrm{R}^{1}=\mathrm{H}, \mathrm{OH}$ or $\mathrm{COOH}$ $\mathrm{R}^{2}=\mathrm{H}$ or $\mathrm{OH}$<smiles>[X]c1ccc(S(N)(=O)=O)c([R])c1[R]</smiles>

97

Scheme 31. 


$$
\begin{array}{lcc}
\mathrm{ArH}+\mathrm{I}_{2} \frac{\mathrm{K}_{2} \mathrm{~S}_{2} \mathrm{O}_{8}, \mathrm{AcOH}, \mathrm{H}_{2} \mathrm{SO}_{4}, \mathrm{ClCH}_{2} \mathrm{CH}_{2} \mathrm{Cl}, 40{ }^{\circ} \mathrm{C}, 12-30 \mathrm{~h}}{69-73 \%} & \operatorname{Arl}(\mathrm{OAc})_{2} \\
100 & 101 \\
\mathrm{Ar}=\mathrm{Ph}, 4-\mathrm{MeC}_{6} \mathrm{H}_{4}, 4-\mathrm{ClC}_{6} \mathrm{H}_{4}, 4-\mathrm{BrC}_{6} \mathrm{H}_{4}, 4-\mathrm{FC}_{6} \mathrm{H}_{4} & \\
\quad \text { Scheme 32. } &
\end{array}
$$




$$
\mathrm{Phl}(\mathrm{OAc})_{2}+2 \mathrm{RCO}_{2} \mathrm{H} \quad \stackrel{\mathrm{PhCl} \text {, heat }}{\stackrel{-2 \mathrm{HOAc}}{\longrightarrow}} \quad \begin{gathered}
\mathrm{Phl}(\mathrm{OCOR})_{2} \\
\mathbf{1 0 2}
\end{gathered}
$$<smiles>CC(=O)C(CCC(=O)OI(C)c1ccccc1)NP</smiles>

103

$$
\mathrm{P}=\mathrm{Cbz} \text { or } \mathrm{Boc}
$$<smiles>CC1(I)C=CC(=O)OC(=[Al])C=C1</smiles>

105

$$
\mathrm{Ar}=4-\mathrm{MeOC}_{6} \mathrm{H}_{4}
$$

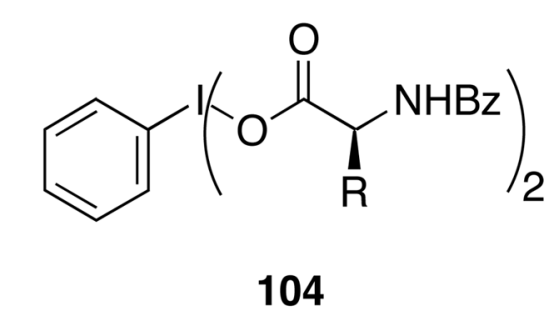

$\mathrm{R}=\mathrm{Me}, \mathrm{CH}_{2} \mathrm{Ph}, \mathrm{CH}\left(\mathrm{CH}_{3}\right)_{2}$, $\mathrm{CH}_{2} \mathrm{CH}\left(\mathrm{CH}_{3}\right)_{2}, \mathrm{CH}\left(\mathrm{CH}_{3}\right) \mathrm{CH}_{2} \mathrm{CH}_{3}$<smiles>Cc1nonc1C(=O)OI(C)c1ccccc1</smiles>

106

Scheme 33. 


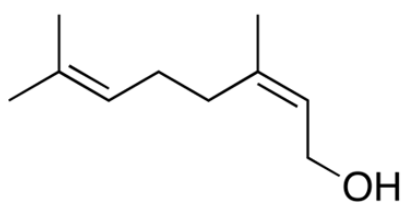

111
$\mathrm{Phl}(\mathrm{OAc})_{2}$ (1.1 equiv.), TEMPO (0.1 equiv.) $\mathrm{MeCN}, \mathrm{H}_{2} \mathrm{O}(\mathrm{pH} 7), 0^{\circ} \mathrm{C}, 20 \mathrm{~min}$

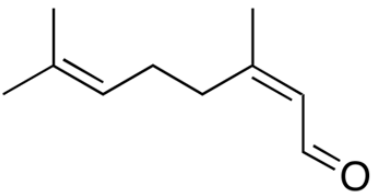

112

Scheme 34. 


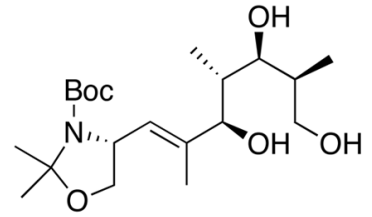

113
$\mathrm{Phl}(\mathrm{OAc})_{2}$ (3.2 equiv.), TEMPO ( 0.2 equiv.) $\mathrm{CH}_{2} \mathrm{Cl}_{2}, \mathrm{rt}, 3.5 \mathrm{~h}$

$95 \%$

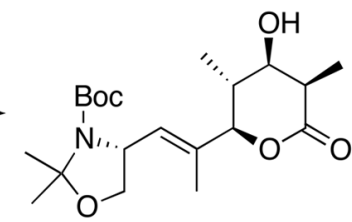

114

Scheme 35 . 


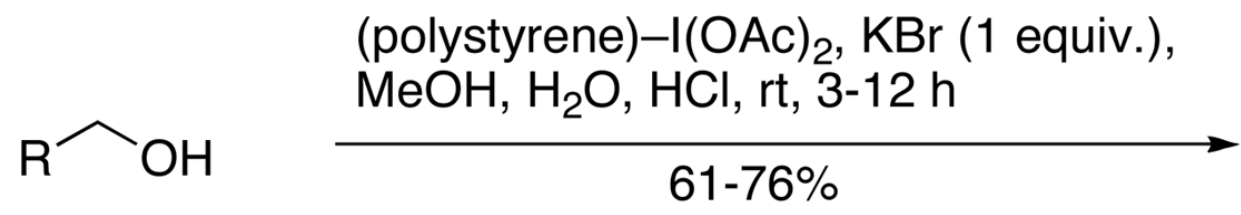

115<smiles>[R]C(=O)OC</smiles>

116

$$
\mathrm{R}=\mathrm{C}_{5} \mathrm{H}_{11} \text {, cylohexyl, Ph, } \mathrm{EtO}_{2} \mathrm{C}\left(\mathrm{CH}_{2}\right)_{4} \text {, etc. }
$$

Scheme 36. 


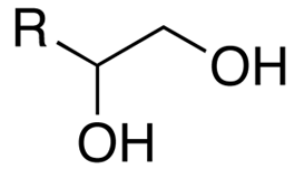

117

$$
\frac{\text { (polystyrene)-I(OAc })_{2}, \mathrm{CH}_{2} \mathrm{Cl}_{2}, \mathrm{rt}, 6-8 \mathrm{~h}}{82-98 \%}
$$

$\mathrm{R}$

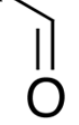

118

Scheme 37. 


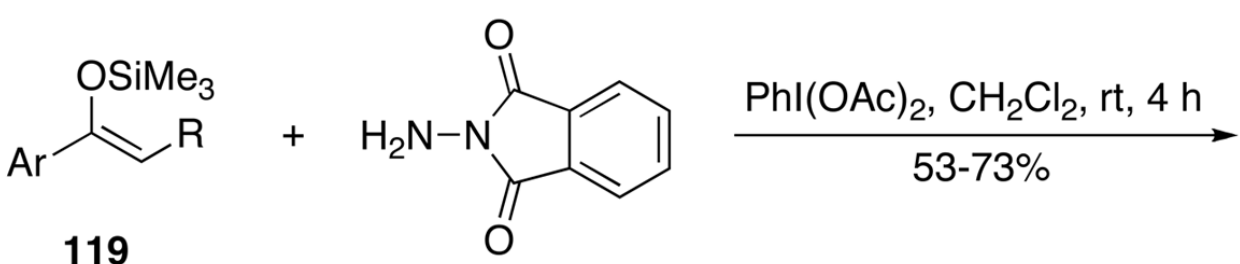

120<smiles>[R]/C(=N\N1C(=O)c2ccccc2C1=O)C(=O)[AlH]</smiles>

121

$\mathrm{Ar}=\mathrm{Ph}, 4-\mathrm{MeC}_{6} \mathrm{H}_{4}, 4-\mathrm{MeOC}_{6} \mathrm{H}_{4}, 4-\mathrm{ClC}_{6} \mathrm{H}_{4}, 4-\mathrm{FC}_{6} \mathrm{H}_{4}, 2,4-\mathrm{Me}_{2} \mathrm{C}_{6} \mathrm{H}_{3}, 2-\mathrm{ClC}_{6} \mathrm{H}_{4}$, etc. $\mathrm{R}=\mathrm{H}, \mathrm{Me}, \mathrm{COOEt}$

Scheme 38 . 


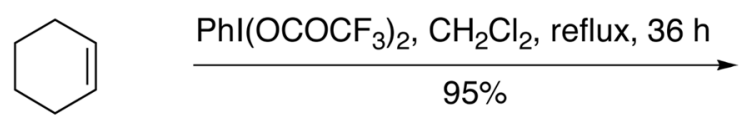<smiles>CCOC(=O)OC1CCCC[C@@H]1OC(F)(F)F</smiles>

122
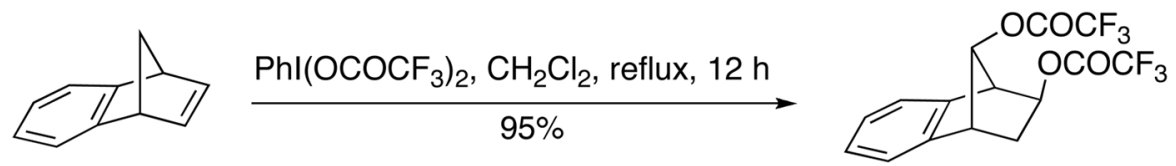

123

124

Scheme 39. 


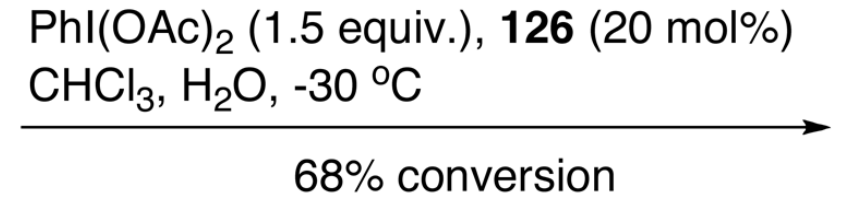

125<smiles>O=C/C=C/c1ccccc1</smiles><smiles>C1CCCCCC1</smiles><smiles>O=C[C@H]1O[C@H]1c1ccccc1</smiles>

127 (84\% ee)

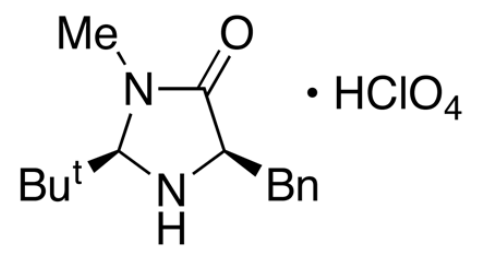

Scheme 40. 


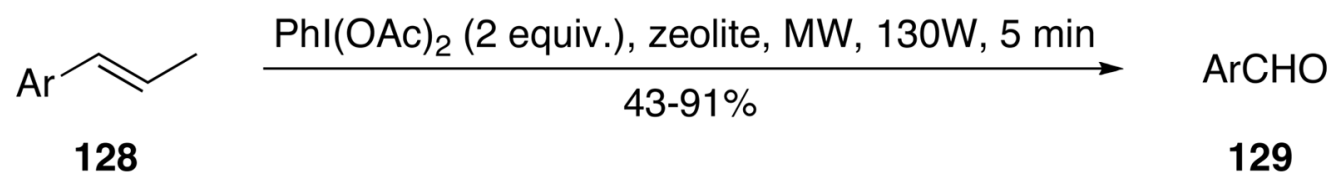

$\mathrm{Ar}=3,4-\left(\mathrm{OCH}_{2} \mathrm{O}\right) \mathrm{C}_{6} \mathrm{H}_{3}, 4-\mathrm{MeOC}_{6} \mathrm{H}_{4}, 3-\mathrm{Me}-4-\mathrm{HOC}_{6} \mathrm{H}_{3}$

Scheme 41. 

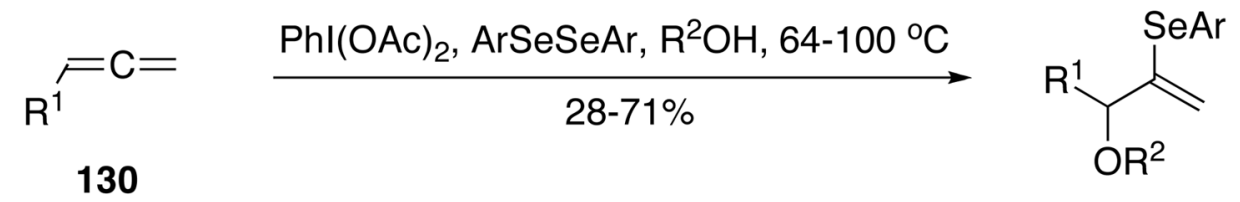

$$
\begin{aligned}
& \mathrm{R}^{1}=\mathrm{Ph}, 4-\mathrm{MeC}_{6} \mathrm{H}_{4}, 2-\mathrm{MeC}_{6} \mathrm{H}_{4}, 2,6-\mathrm{Me}_{2} \mathrm{C}_{6} \mathrm{H}_{3}, \alpha-\mathrm{C}_{10} \mathrm{H}_{7} \\
& \mathrm{Ar}=\mathrm{Ph} \text { or } 4-\mathrm{MeC}_{6} \mathrm{H}_{4} ; \mathrm{R}^{2}=\mathrm{Me}, \mathrm{Et}, \mathrm{Pr}^{\mathrm{i}}, \mathrm{Bu}^{\mathrm{t}}, \mathrm{Ac}, \mathrm{C}_{3} \mathrm{H}_{7} \mathrm{CO}
\end{aligned}
$$

Scheme 42. 


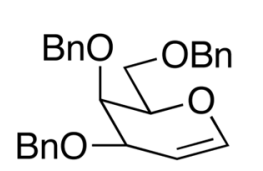

$\mathrm{Phl}(\mathrm{OAc})_{2}, \mathrm{PhSeSePh}, \mathrm{TMSN}_{3}$
$\underset{\mathrm{CH}_{2} \mathrm{Cl}_{2},-30 \text { to }-10^{\circ} \mathrm{C}, 2.5 \mathrm{~h}}{\longrightarrow}$

132

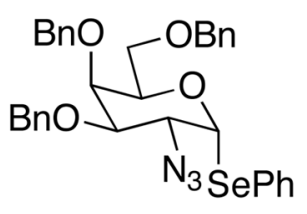

133

Scheme 43. 


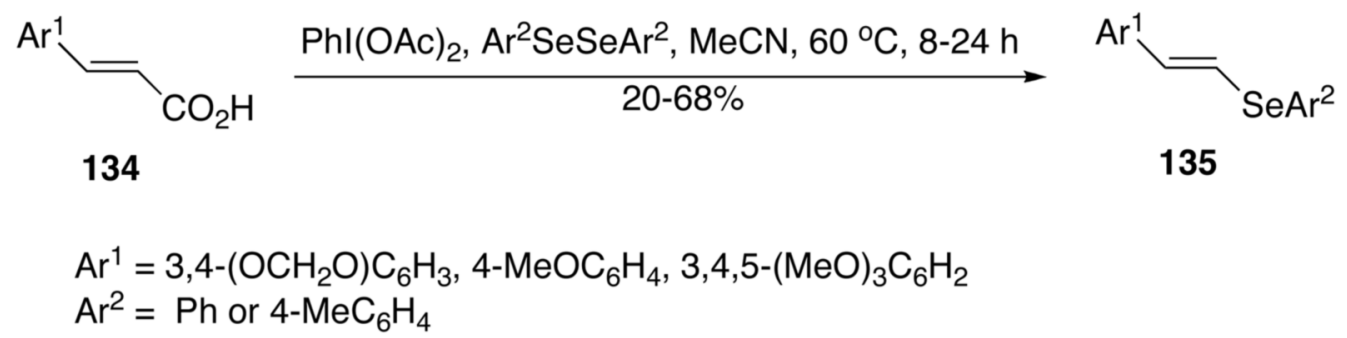

Scheme 44. 


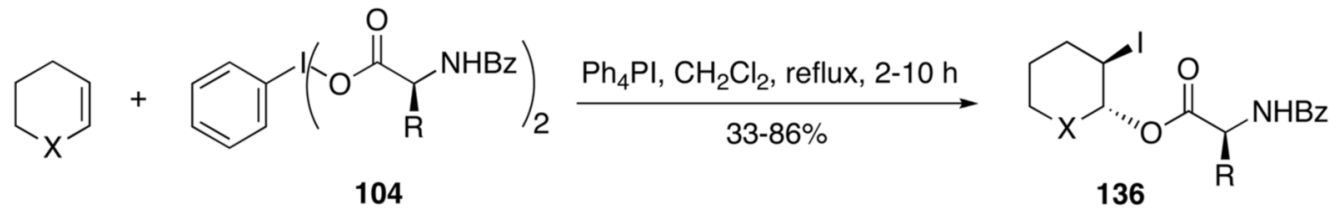
$\mathrm{X}=\mathrm{CH}_{2}$ or $\mathrm{O} ; \mathrm{R}=\mathrm{Me}, \mathrm{CH}_{2} \mathrm{Ph}, \mathrm{CH}\left(\mathrm{CH}_{3}\right)_{2}, \mathrm{CH}_{2} \mathrm{CH}\left(\mathrm{CH}_{3}\right)_{2}, \mathrm{CH}\left(\mathrm{CH}_{3}\right) \mathrm{CH}_{2} \mathrm{CH}_{3}$

Scheme 45. 

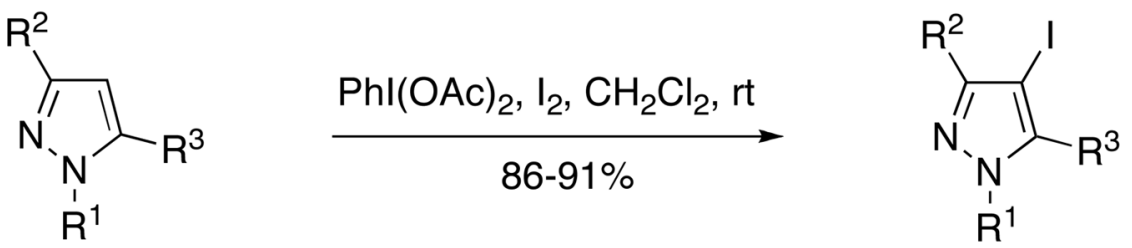
$137 \quad \mathrm{R}^{1}=\mathrm{H}, \mathrm{Ph}, 2,4-\left(\mathrm{NO}_{2}\right)_{2} \mathrm{C}_{6} \mathrm{H}_{3}, 4-\mathrm{ClC}_{6} \mathrm{H}_{4}$ $\mathrm{R}^{2}=\mathrm{Me}$ or $\mathrm{Ph} ; \mathrm{R}^{3}=\mathrm{Me}$ or $\mathrm{Ph}$

Scheme 46. 
<smiles>[R7]c1cn([3H])c([R7])c1[R9]</smiles>

139
$\mathrm{Phl}\left(\mathrm{OCOCF}_{3}\right)_{2}, \mathrm{TMSCN} \mathrm{BF}_{3} \cdot \mathrm{OEt}_{2}, \mathrm{CH}_{2} \mathrm{Cl}_{2}, \mathrm{rt}$ $86-91 \%$<smiles>[R7]c1c([R])c(C#N)n([Y5])c1[R]</smiles>

140

$\mathrm{R}^{1}=\mathrm{H}$ or Me; $\mathrm{R}^{2}=\mathrm{H}$ or $\mathrm{Et}$

$\mathrm{R}^{3}=\mathrm{H}, \mathrm{Me}, \mathrm{Et}, \mathrm{C}_{7} \mathrm{H}_{15}, \mathrm{Bu}^{\mathrm{t}},\left(\mathrm{CH}_{2}\right)_{3} \mathrm{CO}_{2} \mathrm{Et}, 4-\mathrm{BrC}_{6} \mathrm{H}_{4}, 2-\mathrm{BrC}_{6} \mathrm{H}_{4}, 4-\mathrm{MeOC}_{6} \mathrm{H}_{4}$ Scheme 47. 


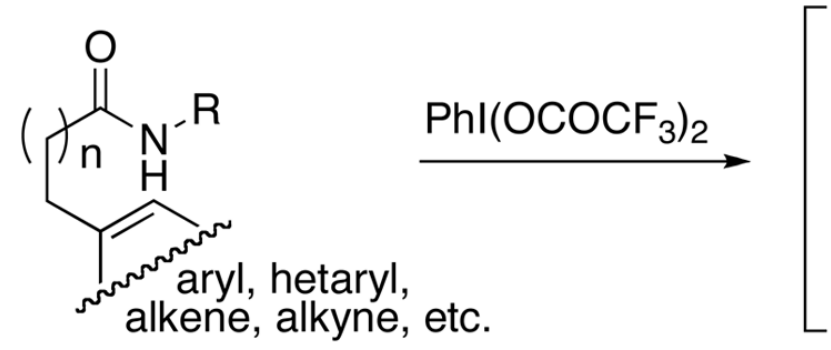

141

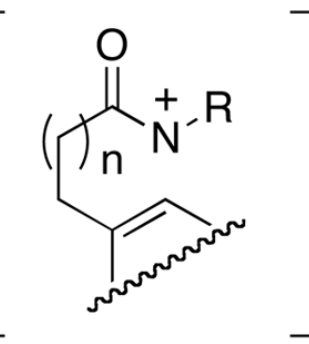

142

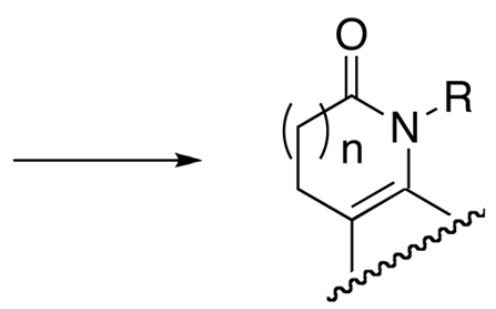

143

$\mathrm{R}=\mathrm{OMe}$, alkyl, Bn, Ph, Ts, Bz, etc.

$\mathrm{n}=0,1,2$

Scheme 48. 
<smiles>[R16]Nc1c([R7])cc([R7])cc1CC=C</smiles>

144<smiles>CC(C(=O)OCc1ccccc1)(C(=O)OCc1ccccc1)C(F)(F)F</smiles>

$\mathrm{R}^{1}=\mathrm{OMe}, \mathrm{Et}, \mathrm{Br}$, or $\mathrm{H} ; \mathrm{R}^{2}=\mathrm{H}$ or $\mathrm{Et}$<smiles>[R7]c1cc([R7])c2c(c1)CC(CO)N2C(=O)c1ccccc1</smiles>

145

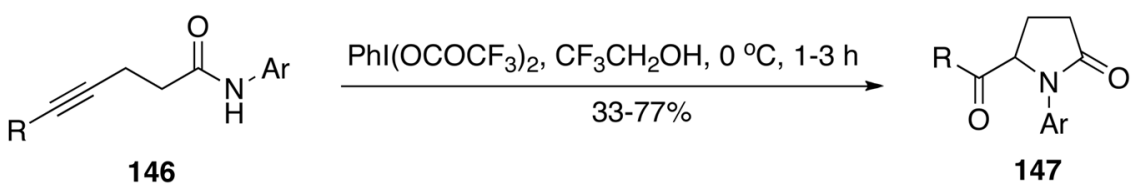

$\mathrm{R}=\mathrm{Ph}, 2-\mathrm{MeC}_{6} \mathrm{H}_{4}, 4-\mathrm{MeOC}_{6} \mathrm{H}_{4}, 4-\mathrm{ClC}_{6} \mathrm{H}_{4}$, 2-thienyl, $\mathrm{PhCH}=\mathrm{CH}_{2}$, etc $\mathrm{Ar}=4-\mathrm{MeOC}_{6} \mathrm{H}_{4}$

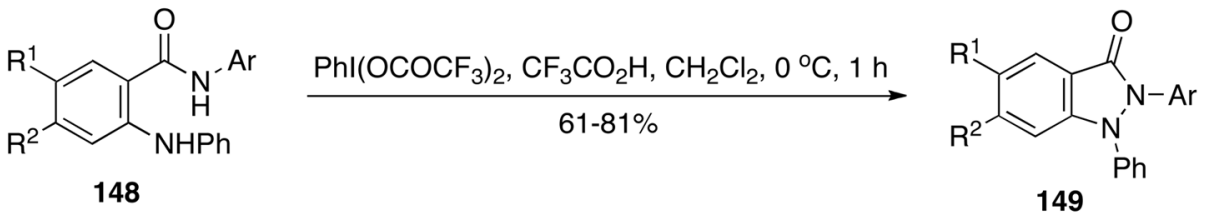

$\mathrm{R}^{1}=\mathrm{H}$ or $\mathrm{F} ; \mathrm{R}^{2}=\mathrm{H}$ or $\mathrm{Cl}$

$\mathrm{Ar}=4-\mathrm{MeOC}_{6} \mathrm{H}_{4}$

Scheme 49. 
<smiles>[R]C(=O)CC([R7])=O</smiles>

150

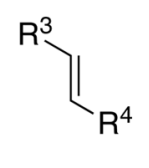

151
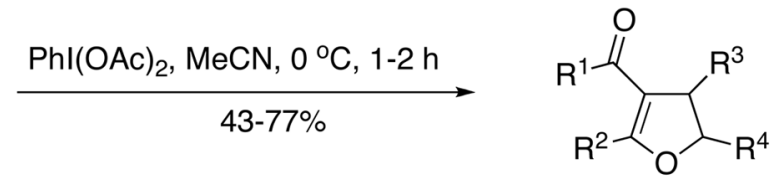

152

$R^{1}=M e, R^{2}=M e$

$\mathrm{R}^{1}=\mathrm{Me}, \mathrm{R}^{2}=\mathrm{OEt}$

$\mathrm{R}^{3}$ and $\mathrm{R}^{4}=\mathrm{H}$, alkyl, $\mathrm{Ph}, \mathrm{CH}_{2} \mathrm{OAc}, \mathrm{CH}_{2} \mathrm{Cl}$, etc.

\section{Scheme 50.}


<smiles>C=C(P)CCC(=O)O</smiles>

153

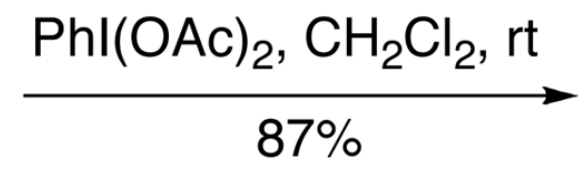

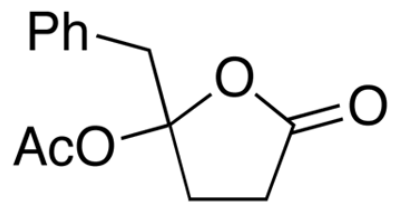

154

Scheme 51. 
<smiles>[R7]C1([R])C[C@@H](O)[C@H]2O[C@]2([R])C1</smiles>

$\underset{53-74 \%}{\stackrel{\mathrm{Phl}\left(\mathrm{OCOCF}_{3}\right)_{2}, \mathrm{MeCN} / \mathrm{H}_{2} \mathrm{O} 4: 1,0{ }^{\circ} \mathrm{C} \text { to rt }}{\longrightarrow}}$<smiles>[R]C1([R])C[C@@H]2C[C@@]([R])(O1)[C@H](O)O2</smiles>

156

$\mathrm{R}=\mathrm{Me}$, Et, $n-\mathrm{C}_{11} \mathrm{H}_{23}, \mathrm{CH}_{2} \mathrm{CH}\left(\mathrm{CH}_{3}\right)_{2}, \mathrm{CH}_{2} \mathrm{Ph} ; \mathrm{R}^{1}=\mathrm{H}$ or $\mathrm{Me}$

Scheme 52. 


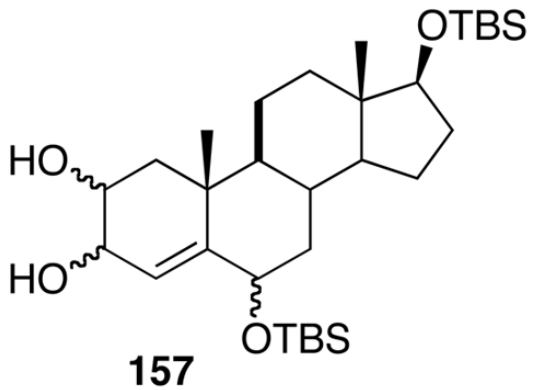

$\underset{90 \%}{\stackrel{\mathrm{Phl}(\mathrm{OAc})_{2 .} \mathrm{MeCN}, \mathrm{rt}, 14 \mathrm{~h}}{\longrightarrow}}$

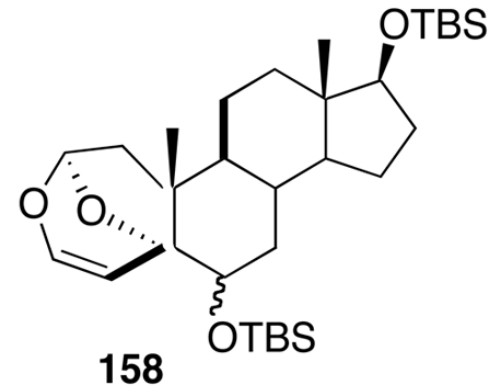

Scheme 53. 


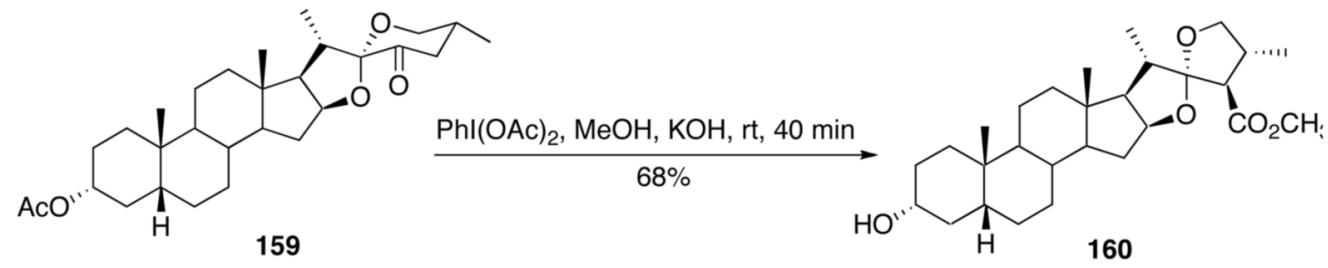

Scheme 54. 

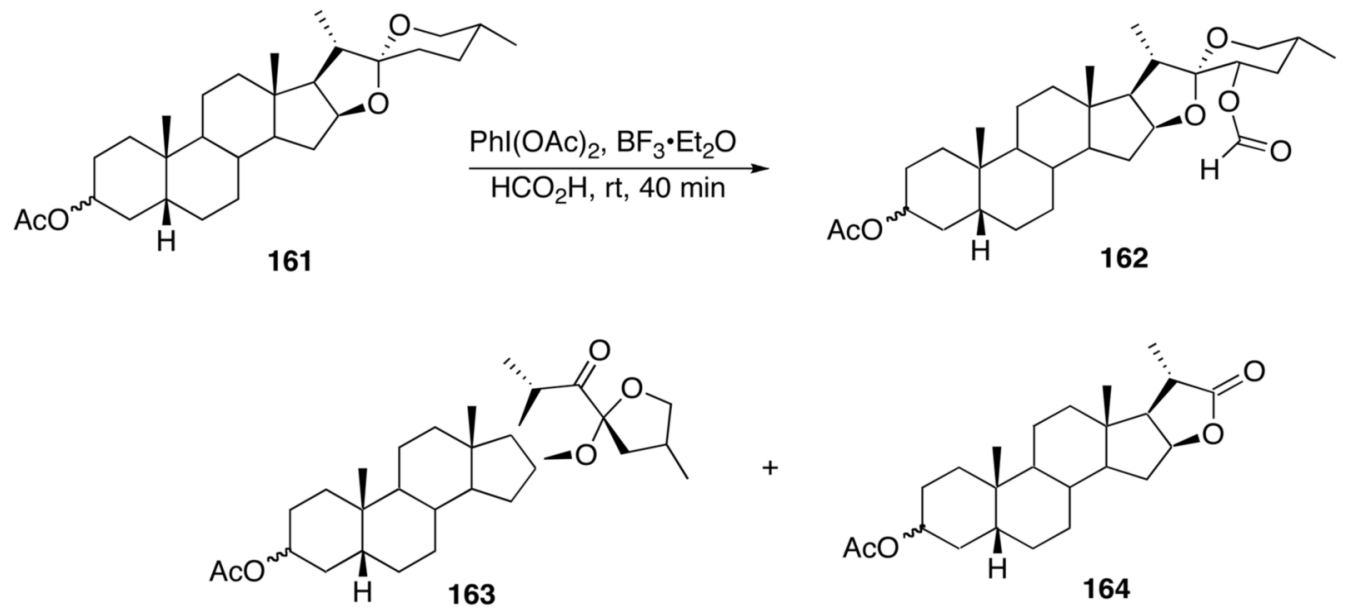

Scheme 55. 

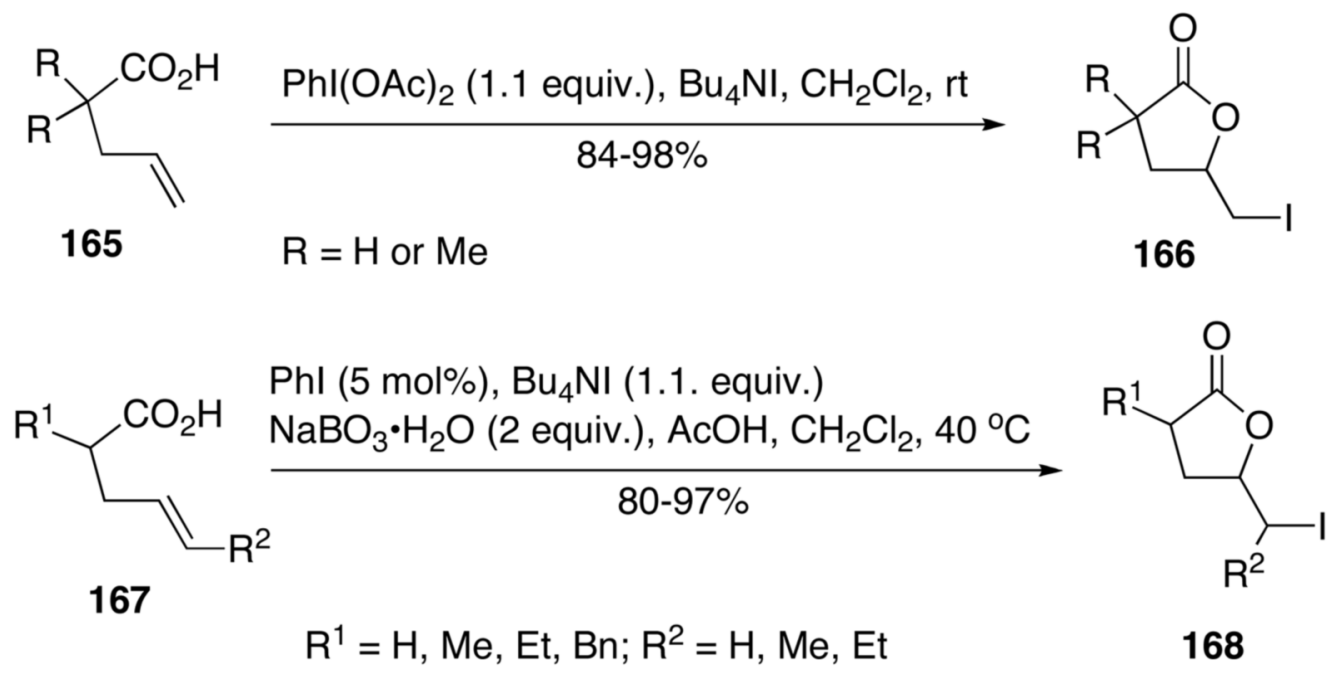

Scheme 56. 


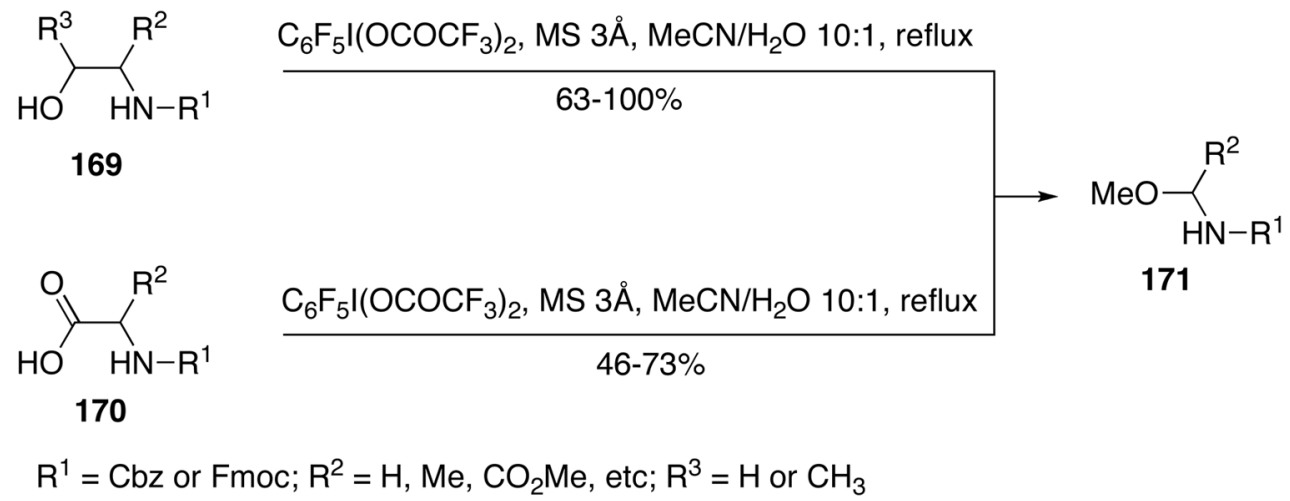

Scheme 57. 
<smiles>C1CNC2CCCNC2C1</smiles>

172

\section{$\mathrm{Phl}(\mathrm{OAc})_{2}, \mathrm{NaOH}, \mathrm{H}_{2} \mathrm{O}$, rt $50 \%$}

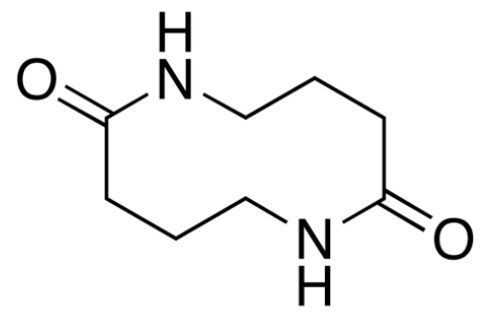

173

Scheme 58. 
$\underset{\sim}{\sim}>{ }^{\mathrm{O}} \mathrm{C}_{6} \mathrm{H}_{13} \frac{\text { Toll }(\mathrm{OAc})_{2}(1.1 \text { equiv. }), \mathrm{CH}_{2} \mathrm{Cl}_{2} / \mathrm{HFIP}, 0^{\circ} \mathrm{C}, 1 \mathrm{~h}}{76 \%}$

174<smiles>CCCCCC[C@@H]1CC[C@](C)(OC(C)=O)CO1</smiles>

175

Scheme 59. 
$\mathrm{H}_{2} \overbrace{\mathrm{CO}_{2} \mathrm{H}}^{\stackrel{\mathrm{Phl}(\mathrm{OAc})_{2}, \mathrm{PrOH}, \mathrm{H}_{2} \mathrm{O}, 50^{\circ} \mathrm{C}, 1.5 \mathrm{~h}}{\longrightarrow}}$ 176<smiles>[R]c1ccc([Y])c(C(N)=O)c1</smiles>

178

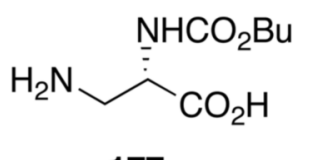

177

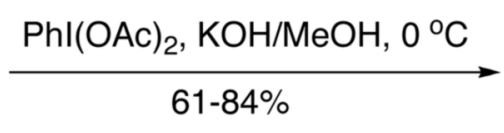<smiles>[R]c1ccc2[nH]c(=O)[nH]c2c1</smiles>

179 $\mathrm{R}=\mathrm{H}$ or $\mathrm{Cl} ; \mathrm{X}=\mathrm{NH}, \mathrm{NMe}, \mathrm{NEt}, \mathrm{NPr}, \mathrm{NPr} \dot{\mathrm{i}}, \mathrm{NBu}, \mathrm{NBn}, \mathrm{O}$<smiles>[R17]n1cc(CC(N)=O)c2ccccc21</smiles>

$\mathrm{Phl}(\mathrm{OAc})_{2}, \mathrm{R}^{2} \mathrm{OH}, \mathrm{rt}, 4-24 \mathrm{~h}$ $70-99 \%$

$\mathrm{R}^{1}=$ Boc or Ts; $\mathrm{R}^{2}=\mathrm{Me}, \mathrm{Et}, \mathrm{Pr}^{\mathrm{i}}, \mathrm{Bu}^{\mathrm{t}}, \mathrm{Bn}$<smiles>[R]C(=O)NCc1cn([Tl])c2ccccc12</smiles>

Scheme 60. 


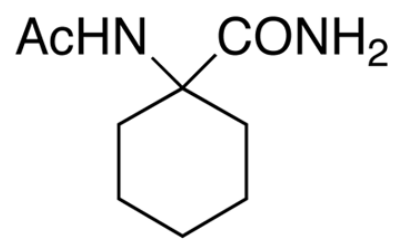

$\underset{83 \%}{\stackrel{\mathrm{Phl}\left(\mathrm{OCOCF}_{3}\right)_{2}, \mathrm{MeCN}, \mathrm{H}_{2} \mathrm{O}, \mathrm{rt}, 1 \mathrm{~h}}{\longrightarrow}}$

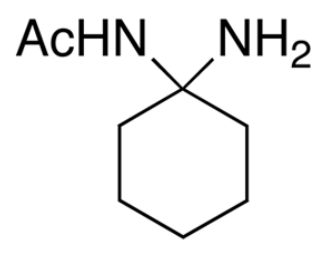

183

Scheme 61. 


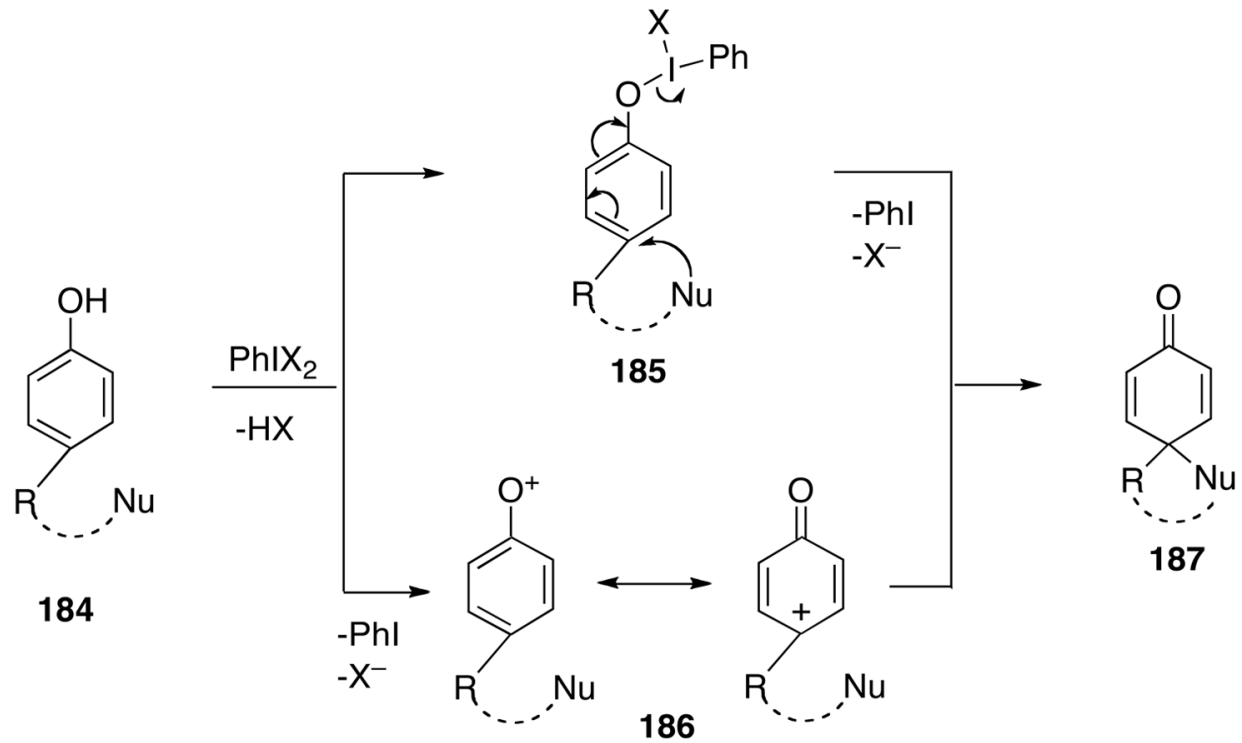<smiles>[R]c1ccccc1O</smiles><smiles></smiles><smiles>[R]C1([N])C=CC=CC1=O</smiles><smiles>[X]OC(=O)OOOC(F)(F)F</smiles>

Scheme 62. 
<smiles>[R]c1ccc(O)cc1</smiles>

$$
\frac{\mathrm{Phl}\left(\mathrm{OCOCF}_{3}\right)_{2} \text { or } \mathrm{Phl}(\mathrm{OAc})_{2}}{\mathrm{CH}_{2} \mathrm{Cl}_{2}, \mathrm{Py} \cdot(\mathrm{HF})_{x}, \mathrm{rt}, 30 \mathrm{~min}}
$$<smiles>[R]C1(F)C=CC(=O)C=C1</smiles>

\section{0}

$\mathrm{R}=\mathrm{Me}, \mathrm{Et}, \mathrm{CH}_{2} \mathrm{CH}_{2} \mathrm{Br}, \mathrm{F}, \mathrm{Cl}$, etc.<smiles>[R]C1CCc2ccc(O)cc2C1</smiles>

$\underset{\mathrm{Phl}(\mathrm{OAc})_{2} \text { (1equiv.), } \mathrm{MeOH}, 0{ }^{\circ} \mathrm{C}, 1-1.5 \mathrm{~h}}{\longrightarrow}$<smiles>[R]C1CCC2(OC)C=CC(=O)C=C2C1</smiles>

191

$$
\mathrm{n}=0 \text { or } 1 ; \mathrm{R}=\mathrm{H}, \mathrm{Me}, \mathrm{Et} \text {, etc. }
$$<smiles>[R6]c1ccc(OS(C)(=O)=O)c([R])c1</smiles>

192

$\mathrm{R}^{2}$

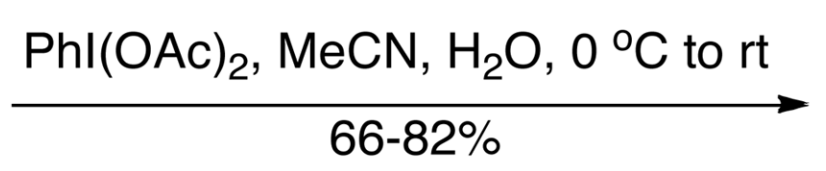

$$
\mathrm{R}^{1}=\mathrm{H} \text { or } \mathrm{Br} ; \mathrm{R}^{2}=\mathrm{Me}, \mathrm{Ph}, 2-\mathrm{FC}_{6} \mathrm{H}_{4}, 3-\mathrm{NO}_{2} \mathrm{C}_{6} \mathrm{H}_{4}
$$<smiles>[R7]C1=CC([R2])(O)C=CC1=O</smiles>

193 Scheme 63. 
<smiles>COc1ccc2ccccc2c1O</smiles>

194

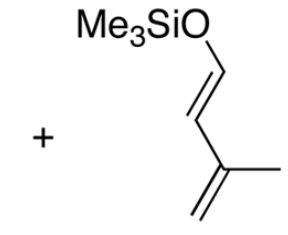

195

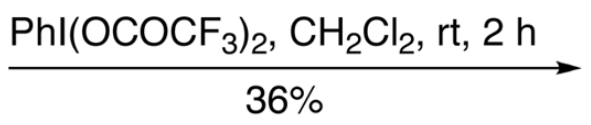

$196(E / Z 1.3: 1)$

Scheme 64. 

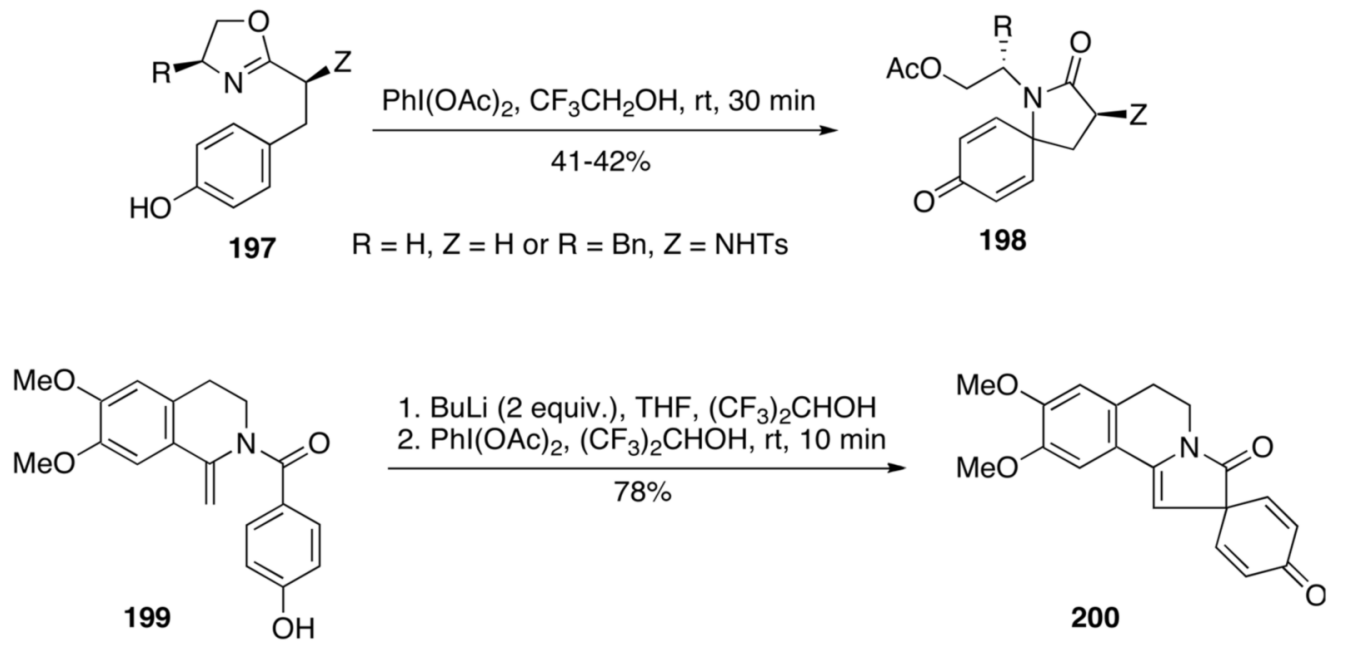<smiles>C[C@H]1CCCC2(C)[C@H](Cc3ccc(O)c(O)c3)[C@H](O)CC[C@H]12</smiles>

201

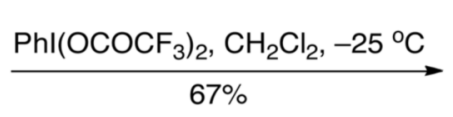<smiles>C[C@H]1CCCC2(C)[C@@H]1CC[C@]1(C)OC3(C=CC(=O)C(O)=C3)C[C@H]21</smiles>

202

Scheme 65. 

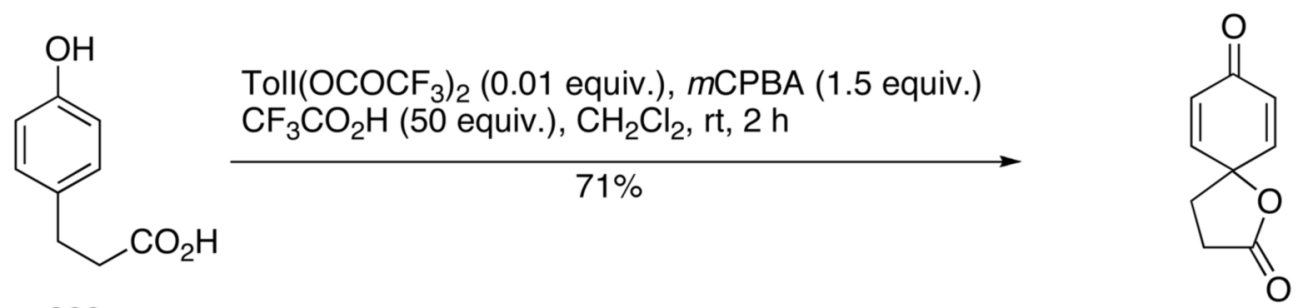

203

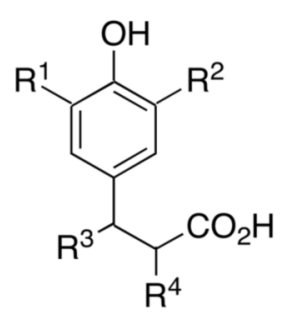

Toll ( 0.05 equiv.), $m$ CPBA ( 1.5 equiv.) $\mathrm{CF}_{3} \mathrm{CO}_{2} \mathrm{H}$ (1 equiv.), $\mathrm{CH}_{2} \mathrm{Cl}_{2}, \mathrm{rt}, 2 \mathrm{~h}$

$$
66-91 \%
$$

205

$\mathrm{R}^{1}, \mathrm{R}^{2}, \mathrm{R}^{3}, \mathrm{R}^{4}=\mathrm{H}, \mathrm{Me}, \mathrm{Br}$

$$
\text { or } \mathrm{R}^{1}, \mathrm{R}^{2}=\mathrm{H}, \mathrm{R}^{3}+\mathrm{R}^{4}=(=\mathrm{CH}-\mathrm{CH}=\mathrm{CH}-\mathrm{CH}=)
$$<smiles>[R]C1=CC2(C=C([R])C1=O)OC(=O)C([R8])C2[R]</smiles>

206

Scheme 66. 

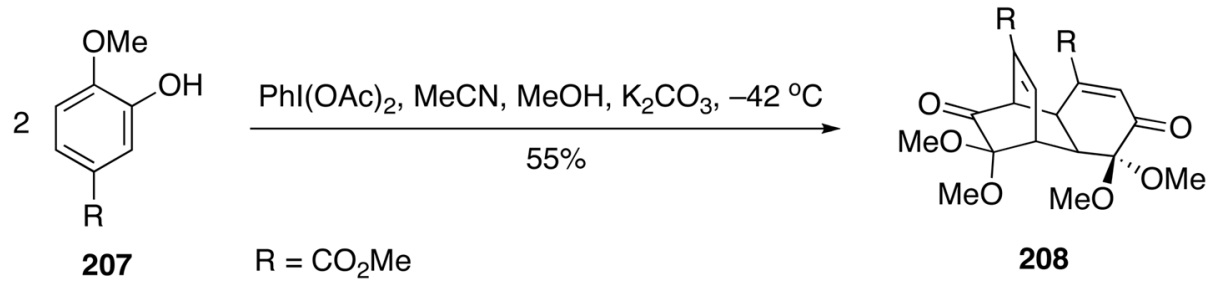

Scheme 67. 
TMS

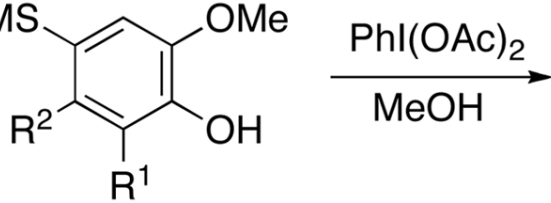

209<smiles>[R]C1=C([R])C(S(C)(=O)=O)=CC(OC)(OC)C1=O</smiles>

210

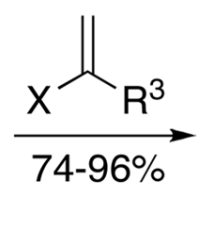

$\underset{41-58 \%}{\stackrel{\mathrm{Phl}\left(\mathrm{OCOCF}_{3}\right)_{2}, \mathrm{rt}}{\longrightarrow}}$

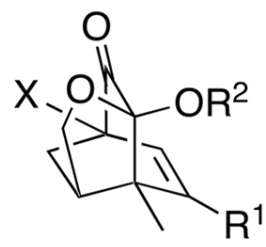

213

$$
\mathrm{X}=\mathrm{Br} \text { or } \mathrm{H} ; \mathrm{R}^{1}=\mathrm{CO}_{2} \mathrm{Me} \text { or OTs; } \mathrm{R}^{2}=\mathrm{Me} \text { or } \mathrm{Bn}
$$

Scheme 68. 


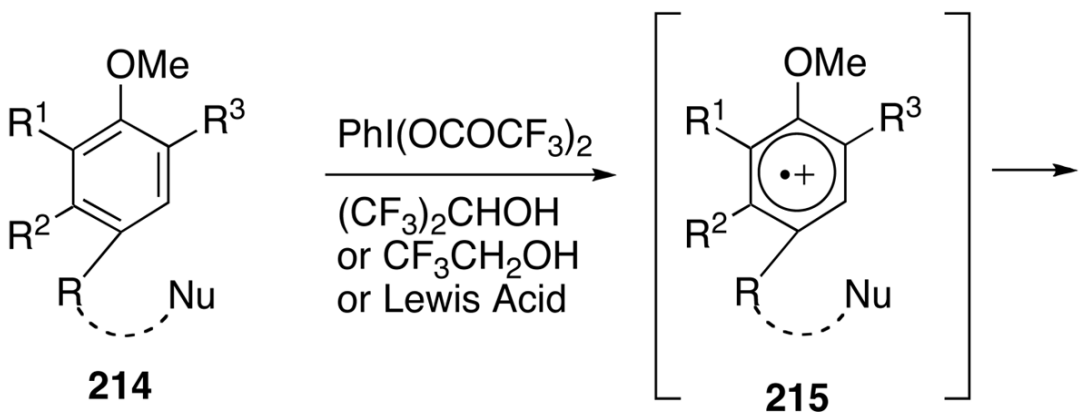<smiles>[R]CCC1([Y])C=C([R])C(=O)C([R])=C1[Hg]</smiles><smiles>[R]c1c([R])c(OC)c([10B])c([R])c1NCC</smiles>

Scheme 69. 


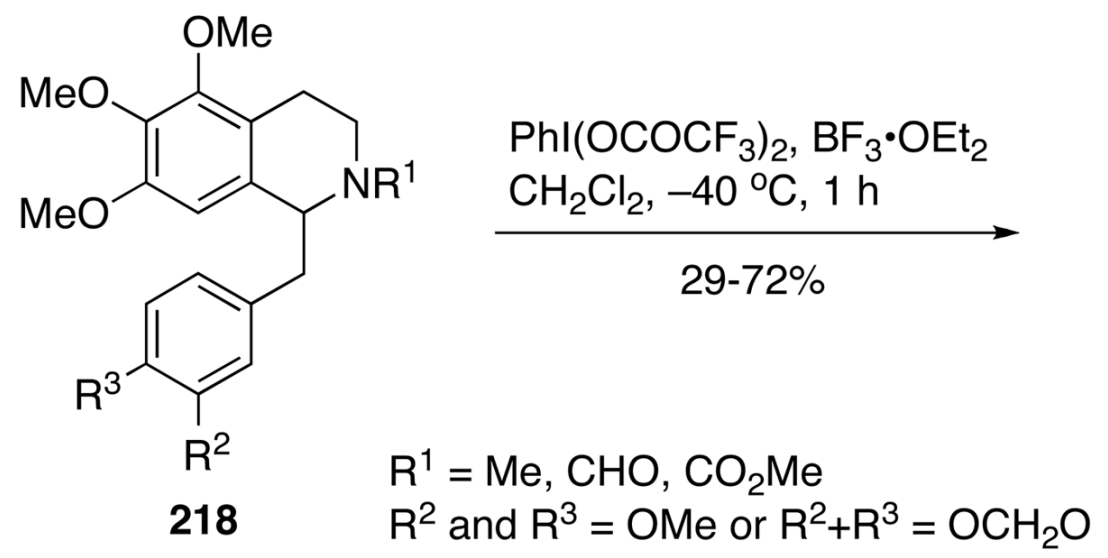<smiles>[R7]c1cc2c(cc1[2H])-c1c(OC)c(OC)c(OC)c3c1C(C2)NCC3</smiles>

219<smiles>COc1ccc(CC(=O)Oc2ccc(OC)c(OC)c2)cc1OC</smiles>

$\mathrm{Phl}\left(\mathrm{OCOCF}_{3}\right)_{2}, \mathrm{BF}_{3} \cdot \mathrm{OEt}_{2}$ $\mathrm{MeCN}, \mathrm{rt}, 10 \mathrm{~min}$
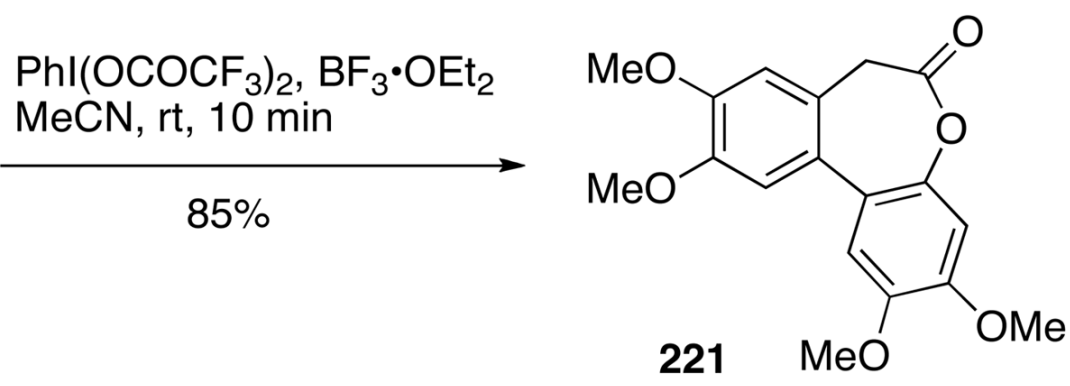<smiles>[Y]Cc1cc([R7])c([R7])c([Y])c1</smiles>

$\mathrm{Phl}\left(\mathrm{OCOCF}_{3}\right)_{2}, \mathrm{H}_{3}\left[\mathrm{PW}_{12} \mathrm{O}_{40}\right]$ $\mathrm{MeCN},-20^{\circ} \mathrm{C}$ to $\mathrm{rt}, 40 \mathrm{~min}$

$\mathrm{R}^{1}, \mathrm{R}^{2}, \mathrm{R}^{3}=\mathrm{H}$ or OMe or $\mathrm{R}^{1}+\mathrm{R}^{2}=\mathrm{OCH}_{2} \mathrm{O}$ and $\mathrm{R}^{3}=\mathrm{H}$ $\mathrm{X}=\mathrm{NCOCF}_{3}$ or $\mathrm{CH}_{2} ; \mathrm{n}=1$ or 2<smiles>[Y]Cc1cc([R1])c([R7])c([R])c1-c1cc(OC)c(OC)cc1I</smiles>

Scheme 70. 

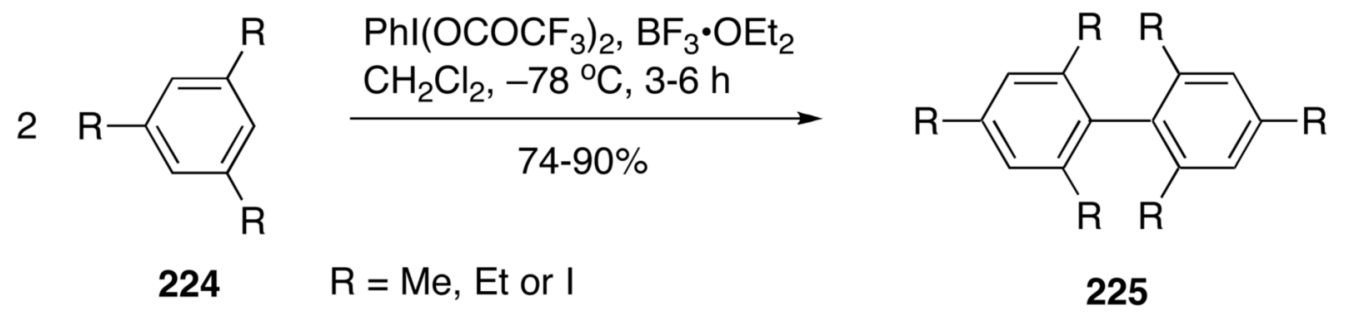

Scheme 71. 


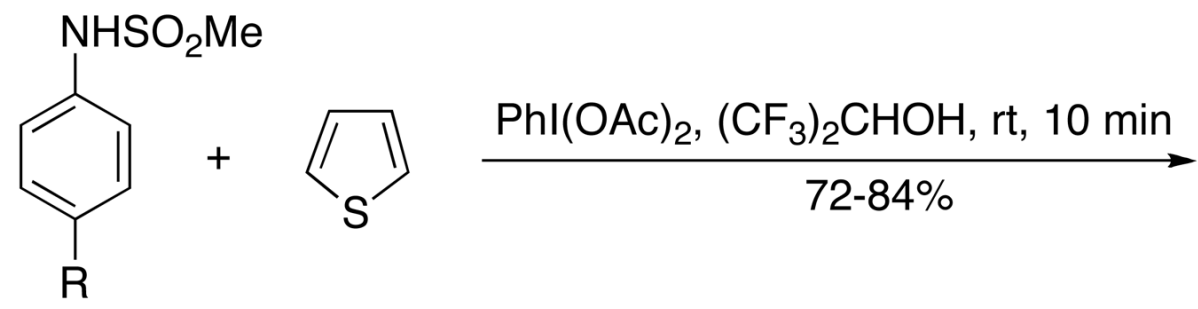

$226 \mathrm{R}=\mathrm{Me}, \mathrm{Pr}, \mathrm{Pr}^{\mathrm{i}}, \mathrm{CH}_{2} \mathrm{CH}_{2} \mathrm{OH}, \mathrm{Cl}$<smiles>[R]OS(=O)(=O)c1ccc([R])cc1-c1cccs1</smiles><smiles>[R]c1ccsc1</smiles>

2- $\mathrm{MeC}_{6} \mathrm{H}_{4} \mathrm{I}\left(\mathrm{OCOCF}_{3}\right)_{2}$, TMSOTf $\mathrm{CH}_{2} \mathrm{Cl}_{2},-78^{\circ} \mathrm{C}, 3 \mathrm{~h}$ $72-98 \%$

$228 \mathrm{R}=\mathrm{Me}, \mathrm{Bu}, \mathrm{Bu} \mathrm{i}^{\mathrm{i}}$, cyclohexyl, TMS, etc.<smiles>[R]c1c[nH]cc1[R]</smiles>

$\mathrm{Phl}\left(\mathrm{OCOCF}_{3}\right)_{2}, \mathrm{TMSBr}$ $\underset{60-75 \%}{\stackrel{\mathrm{CH}_{2} \mathrm{Cl}_{2},-78 \text { to }-40{ }^{\circ} \mathrm{C}, 1 \mathrm{~h}}{\longrightarrow}}$

$230 \mathrm{R}=\mathrm{H}, \mathrm{Et}, \mathrm{Bu}$, etc. Scheme 72.<smiles>[R]c1csc(-c2sccc2[R])c1</smiles><smiles>[R]c1c[nH]c(-c2[nH]cc([R])c2[R])c1[R]</smiles> 
<smiles>[R9]C[C@H]1O[C@H](O)C(F)(F)[C@H](O)[C@H]1[R9]</smiles>

232

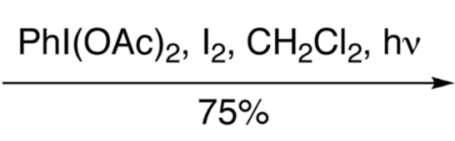

$\mathrm{R}=\mathrm{OAc}$ or $\mathrm{OMe}$<smiles>[R]OC[C@@H](O)[C@@H](O[2H])[C@H](O)C(F)(F)I</smiles>

233

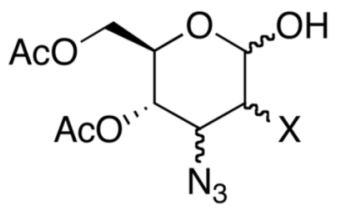

234
$\mathrm{Phl}(\mathrm{OAc})_{2}, \mathrm{I}_{2}, \mathrm{CH}_{2} \mathrm{Cl}_{2}$, hv $81-95 \%$

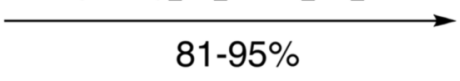

$\mathrm{X}=\mathrm{H}, \mathrm{F}, \mathrm{Cl}, \mathrm{Br}, \mathrm{IC}$

1. $\mathrm{Phl}(\mathrm{OAc})_{2}, \mathrm{I}_{2}, \mathrm{CH}_{2} \mathrm{Cl}_{2}$, hv 2. $\mathrm{Et}_{3} \mathrm{~N}, \mathrm{rt}$ $40-43 \%$

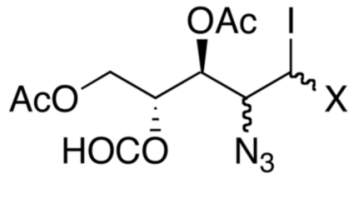

235

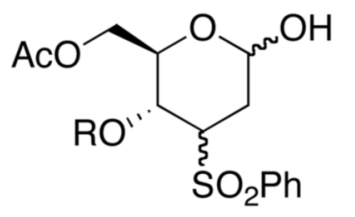

236 $\mathrm{R}=\mathrm{Ac}$ or $\beta-\mathrm{D}-\mathrm{Gal}$

Scheme 73. 


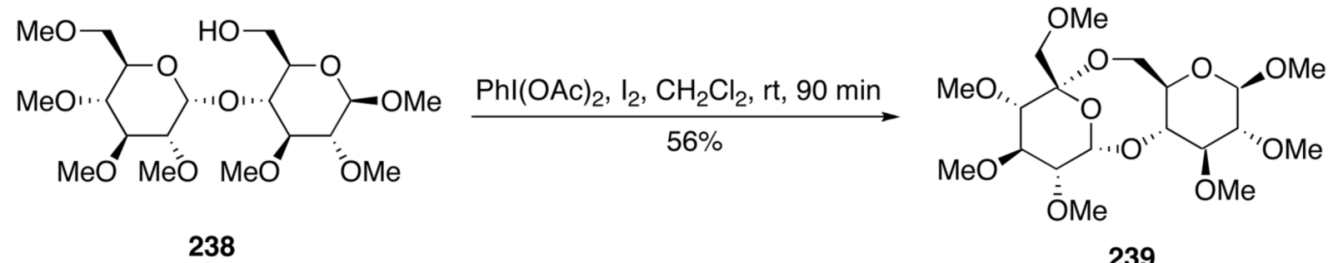

Scheme 74. 


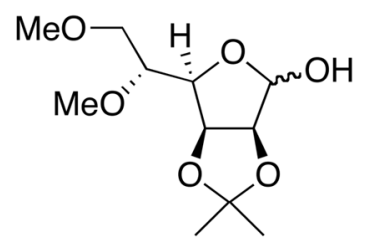

240 $\underset{82 \%}{\stackrel{\mathrm{Phl}(\mathrm{OAC})_{2}, \mathrm{I}_{2}, \mathrm{CH}_{2} \mathrm{Cl}_{2}, \mathrm{rt}, \mathrm{h} v}{\longrightarrow}}$

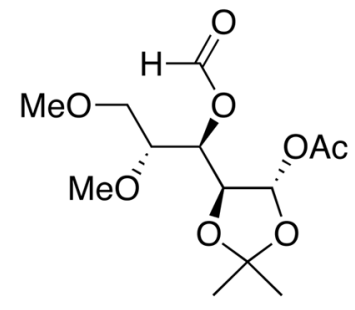

241

Scheme 75. 


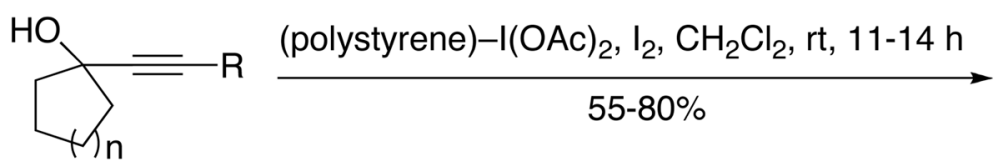

242

$$
\begin{aligned}
& \mathrm{n}=1 ; \mathrm{R}=\mathrm{Ph}, \mathrm{Bu}, \mathrm{TolOCH}_{2} \\
& \mathrm{n}=2 ; \mathrm{R}=\mathrm{Ph}, \mathrm{PhCH}_{2} \mathrm{OCH}_{2}, \mathrm{TolOCH}_{2} \\
& \mathrm{n}=3 ; \mathrm{R}=\mathrm{Ph}, \mathrm{Bu}, \mathrm{TolOCH}_{2}, \mathrm{PhCH}_{2} \mathrm{OCH}_{2}
\end{aligned}
$$

Scheme 76.<smiles>[R]C(I)=C1CCCCC1=O</smiles>

243 


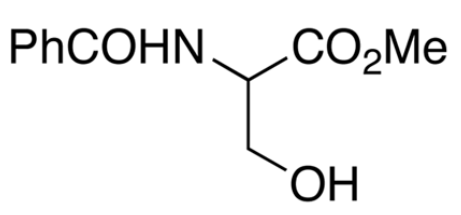

244

$\mathrm{Nu}=\mathrm{CH}_{2}=\mathrm{CHCH}_{2} \mathrm{TMS}$, furan, or elecron-rich aromatics

Scheme 77.

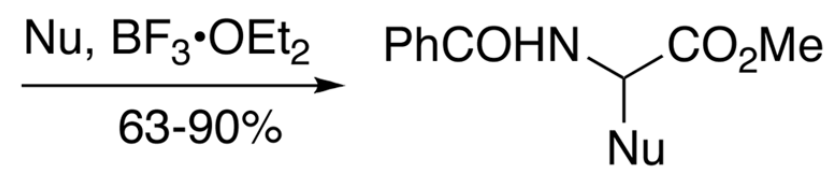

245 

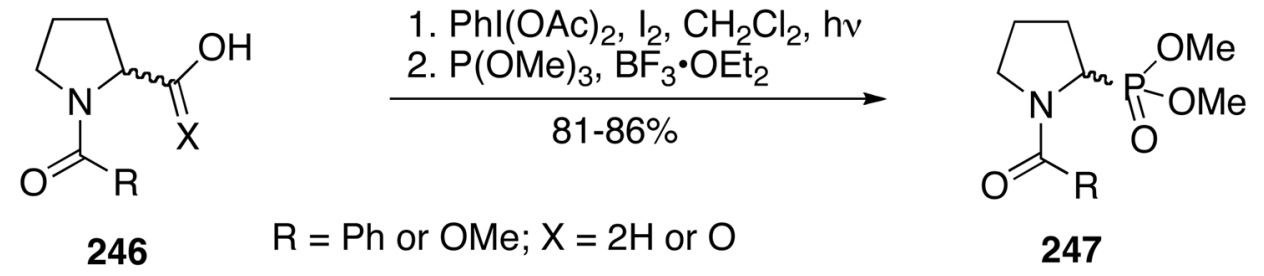

Scheme 78 . 

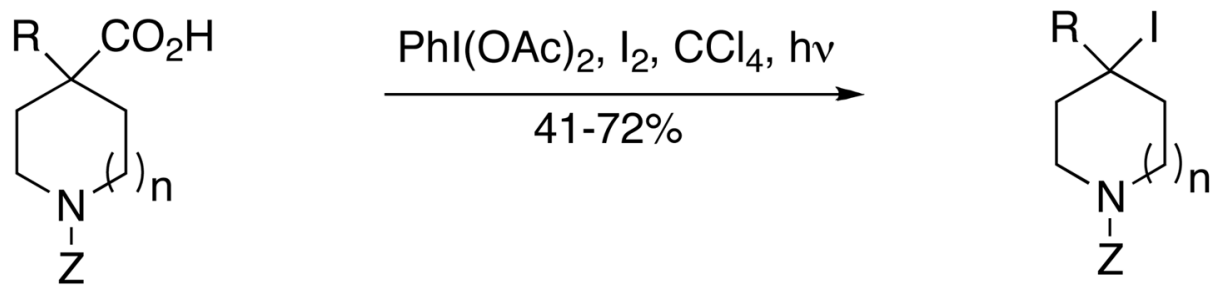

248

$$
\mathrm{n}=0 \text { or } 1 ; \mathrm{R}=\mathrm{H} \text { or } \mathrm{CO}_{2} \mathrm{Me} ; \mathrm{Z}=\mathrm{Boc} \text { or } \mathrm{CO}_{2} \mathrm{Me}
$$

Scheme 79. 

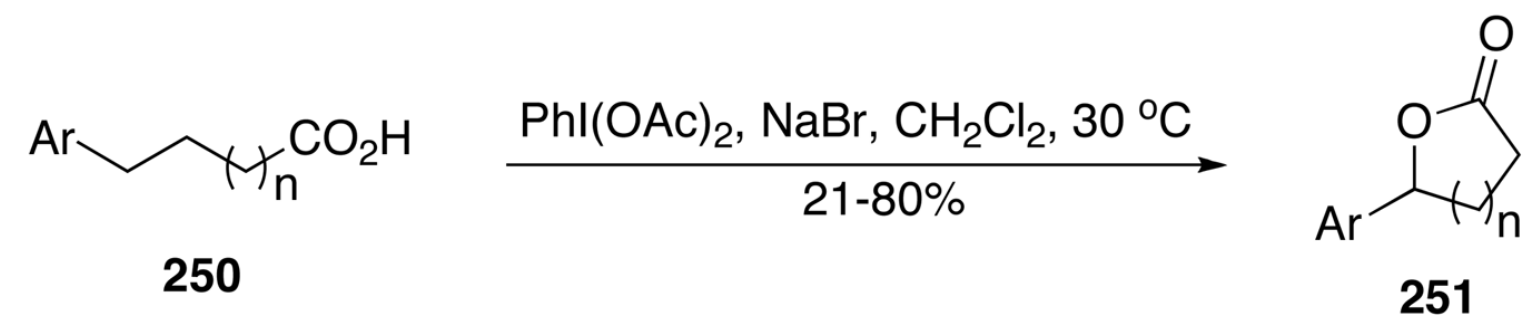

$$
\mathrm{n}=1-3 ; \mathrm{Ar}=\mathrm{Ph}, 4-\mathrm{MeOC}_{6} \mathrm{H}_{4}, 4-\mathrm{NO}_{2} \mathrm{C}_{6} \mathrm{H}_{4}, 4-\mathrm{FC}_{6} \mathrm{H}_{4} \text {, etc. }
$$

Scheme 80. 


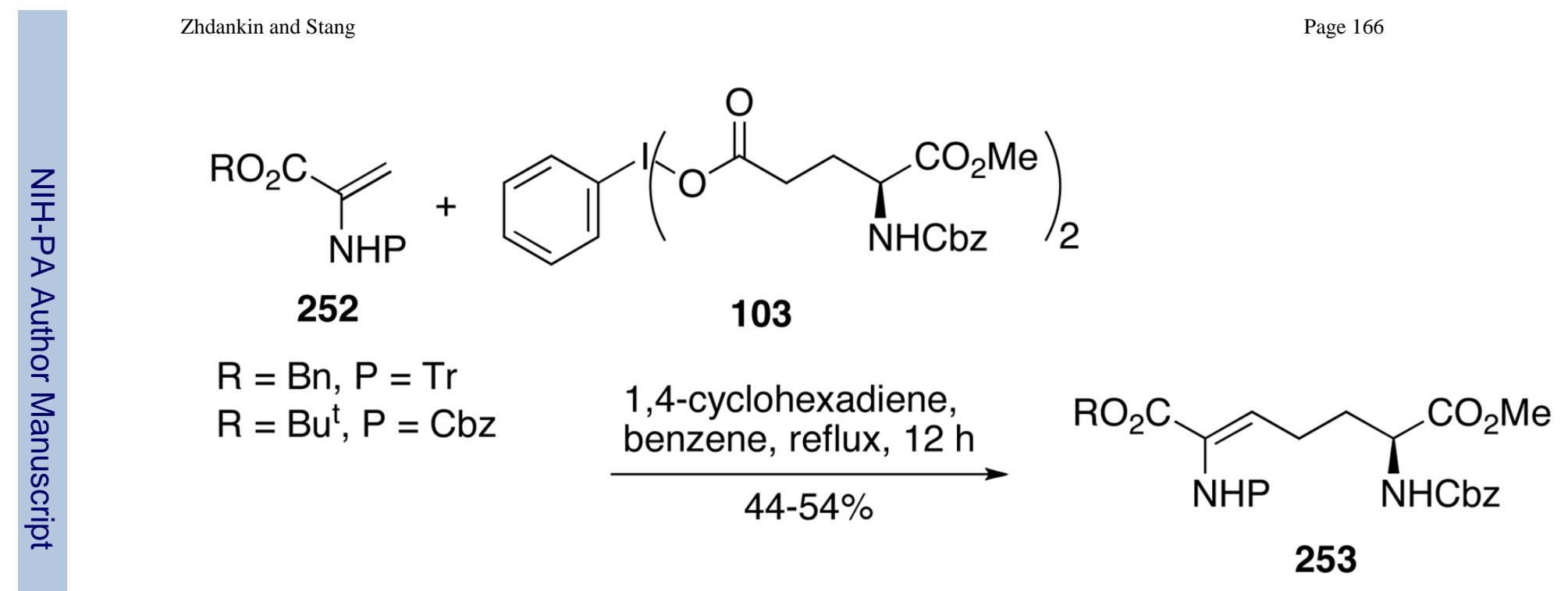

Scheme 81. 
$\mathrm{Phl}(\mathrm{OAc})_{2}$ (1 equiv.), $\mathrm{I}_{2}$ (1.1 equiv.)<smiles>CC1(C)CCCCC1</smiles>
$\mathrm{Bu}^{\mathrm{t}} \mathrm{OH}$ (1 equiv.), $40^{\circ} \mathrm{C}, 1-8 \mathrm{~h}$ $85-98 \%$

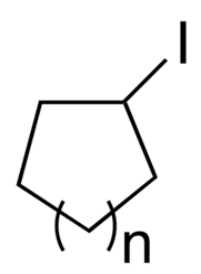

$254 n=1-4$

Scheme 82. 

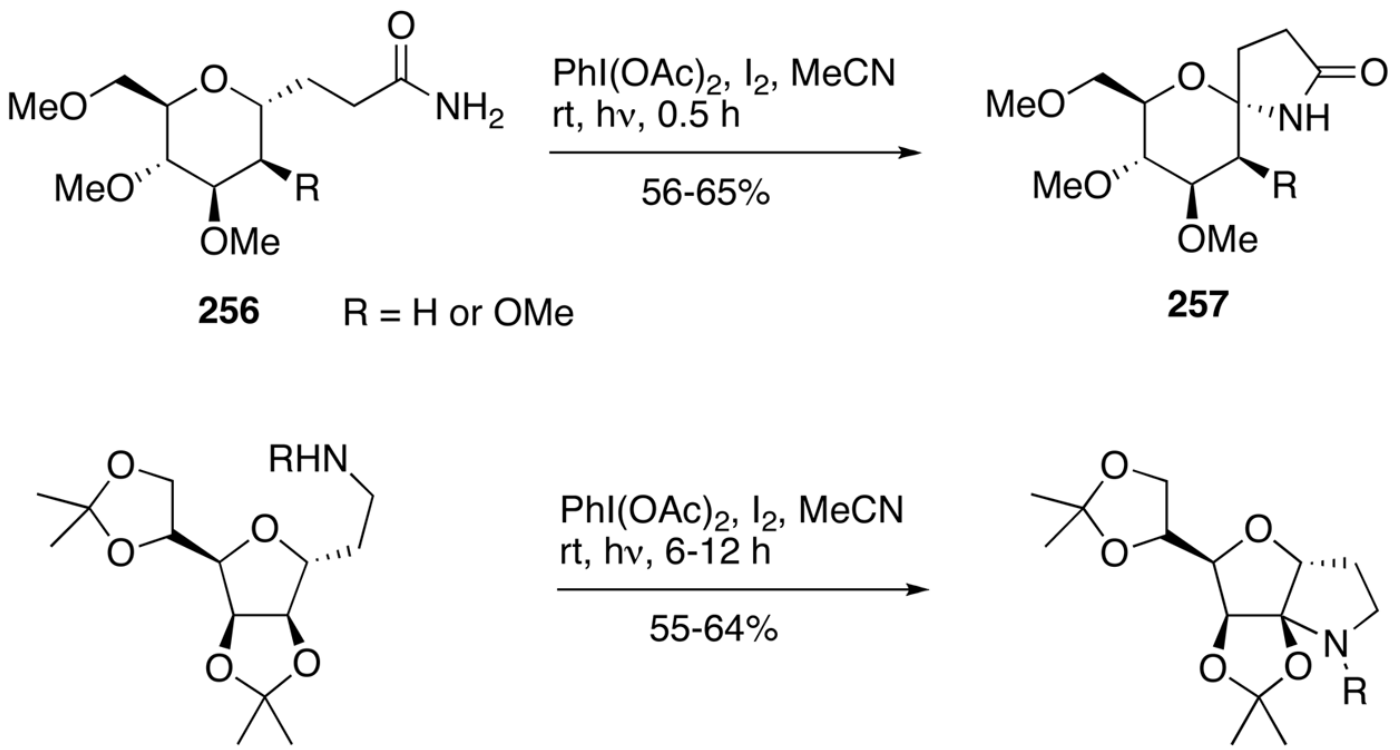

$\mathrm{Phl}(\mathrm{OAc})_{2}, \mathrm{I}_{2}, \mathrm{MeCN}$ $\mathrm{rt}, \mathrm{h} v, 6-12 \mathrm{~h}$ $55-64 \%$

258 $\mathrm{R}=\mathrm{Boc}$ or $\mathrm{PO}(\mathrm{OPh})_{2}$

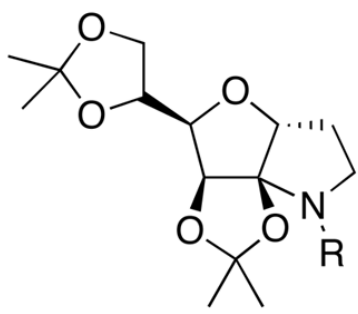

259

Scheme 83. 
<smiles>c1ccc(-c2ccccn2)cc1</smiles>

260<smiles>O=C(N[TeH])OC/C=C\c1ccccc1</smiles>

261
$\mathrm{Phl}(\mathrm{OAc})_{2}$ (1.1-1.6 equiv.), $\mathrm{Pd}(\mathrm{OAc})_{2}(1-6 \mathrm{~mol} \%)$ $\mathrm{MeCN}, 75-100^{\circ} \mathrm{C}, 12-20 \mathrm{~h}$

$$
52 \%
$$

$\mathrm{Phl}(\mathrm{OAc})_{2}$ (2 equiv.), $\mathrm{Pd}(\mathrm{OAc})_{2}(10 \mathrm{~mol} \%)$ $\mathrm{Bu}_{4} \mathrm{NOAc}, \mathrm{MeCN}, 25^{\circ} \mathrm{C}, 7 \mathrm{~h}$

$$
92 \%
$$<smiles>CC(=O)Oc1ccccc1-c1ccccn1</smiles><smiles>CC(=O)OC(c1ccccc1)C1COC(=O)N1[Te]</smiles>

9.5:1 $d r$

Scheme 84. 
$\mathrm{Phl}(\mathrm{OAc})_{2}, \mathrm{PdCl}_{2}$ (2 mol\%), Cul (2 mol\%) $\mathrm{Et}_{3} \mathrm{~N}, \mathrm{THF}, \mathrm{rt}, 0.5-3 \mathrm{~h}$

$2 \mathrm{R} \longrightarrow$

$62-90 \%$

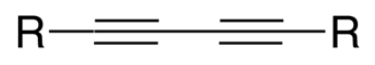

262

263

Scheme 85. 


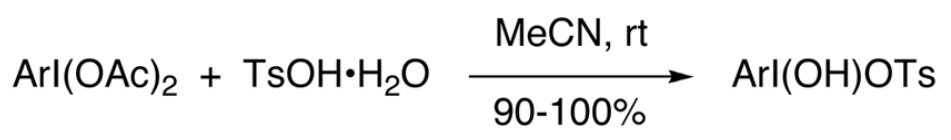
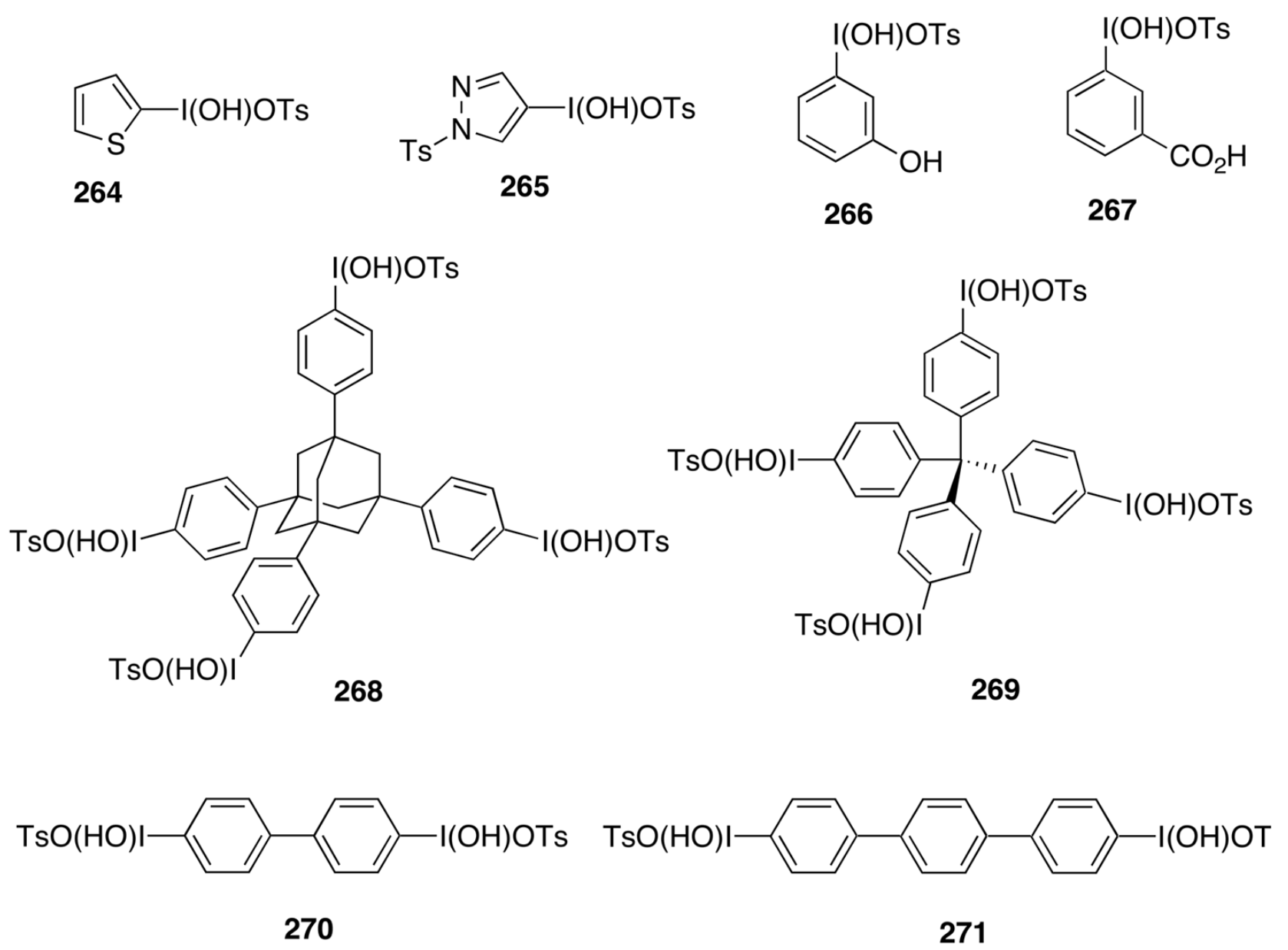

Scheme 86. 


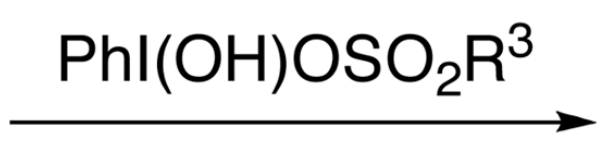<smiles>[R]CC([R7])=O</smiles><smiles>[R6]OC([R])C([R])=O</smiles>

$\mathrm{R}^{1}, \mathrm{R}^{2}=$ alkyl, aryl; $\mathrm{R}^{3}=\mathrm{Me}, p$-Tol, etc.

Scheme 87. 


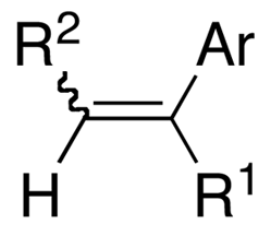

272

\section{$\mathrm{Phl}(\mathrm{OH}) \mathrm{OTs}, 95 \% \mathrm{MeOH}$, rt $70-92 \%$}<smiles>[R2]C(=O)C([R])[AlH2]</smiles>

273

$$
\mathrm{R}^{1}, \mathrm{R}^{2}=\text { alkyl, aryl }
$$

Scheme 88. 


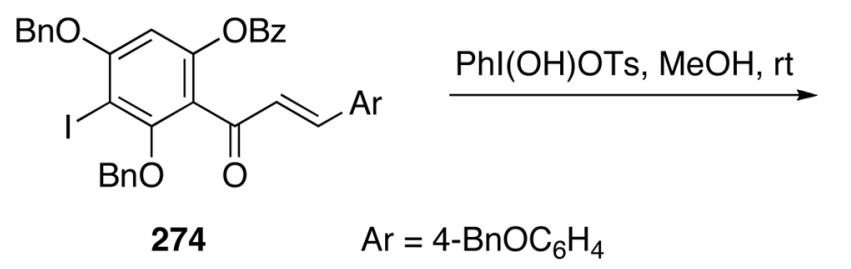<smiles>CC(C)C([Al])C(=O)c1c(OC(=O)c2ccccc2)cc(OCc2ccccc2)c(I)c1O</smiles><smiles>Clc1ccc(C2CC=Cc3ccccc32)cc1Cl</smiles>

276

\section{$\underset{62 \%}{\stackrel{\mathrm{Phl}(\mathrm{OH}) \mathrm{OTs}, \mathrm{MeOH}, \mathrm{rt}}{\longrightarrow}}$}

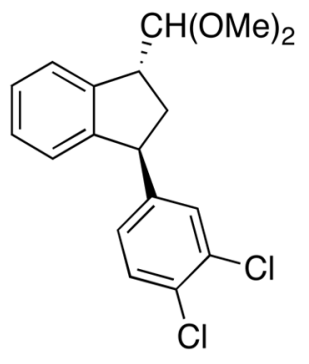

277

Scheme 89. 

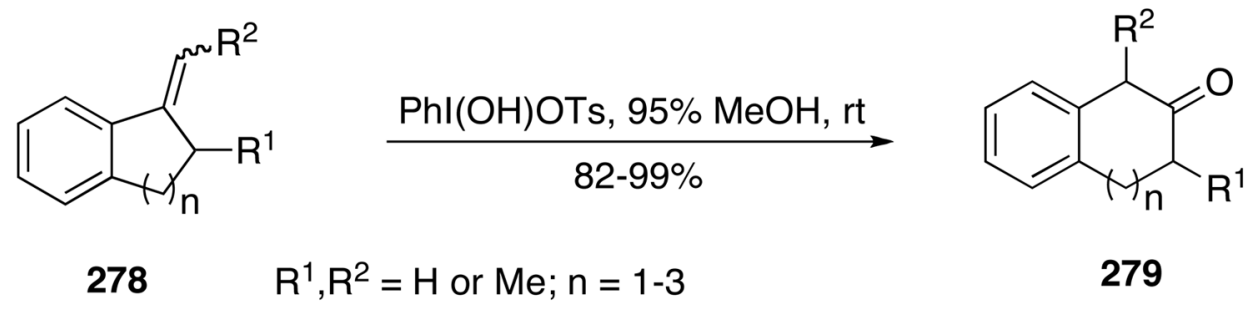

Scheme 90. 


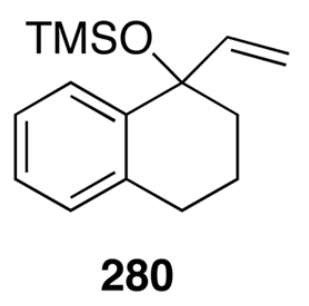

Phl(OH)OTs (2.5 equiv.), $\mathrm{MeOH}, \mathrm{rt}, 2 \mathrm{~h}$ $75 \%$

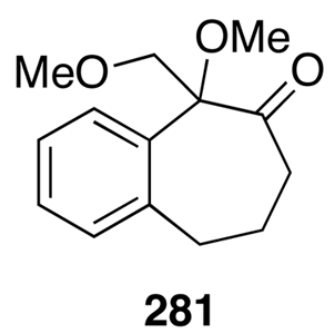

Scheme 91. 
<smiles>c1ccc2cc3ccccc3cc2c1</smiles>

Phl(OH)OTs, $\mathrm{Me}_{3}$ SiNCS, $\mathrm{CH}_{2} \mathrm{Cl}_{2}$, rt, $24 \mathrm{~h}$<smiles>N#Cc1c2ccccc2cc2ccccc12</smiles>

Scheme 92. 


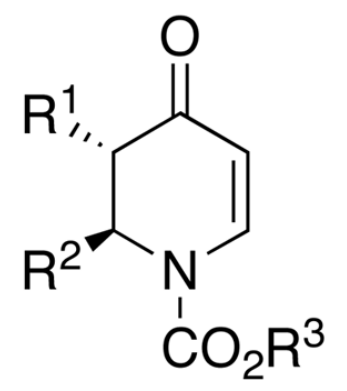

NIS, PhI $(\mathrm{OH}) \mathrm{OTs}$ (0.1 equiv.) $\mathrm{CH}_{2} \mathrm{Cl}_{2}$, rt, dark, $18 \mathrm{~h}$ $67-78 \%$<smiles>[R7]C(=O)N1C=C(I)C(=O)C([R2])[C@H]1[R7]</smiles>

283

Scheme 93. 
$\mathrm{R}^{1}=\mathrm{R}^{2} \frac{\text { (Polystyrene)I(OH)OTs, } \mathrm{I}_{2} \text { or NBS or NCS, } \mathrm{CH}_{2} \mathrm{Cl}_{2}, \mathrm{rt}}{60-97 \%}$

$\mathrm{R}^{1}=\mathrm{Ph}, \mathrm{Bu}, \mathrm{Bu}^{\mathrm{t}}, \mathrm{CH}_{3} \mathrm{OCH}_{2}, \mathrm{H}$

$\mathrm{R}^{2}=\mathrm{H}, \mathrm{Ph}, 4-\mathrm{MeC}_{6} \mathrm{H}_{4} \mathrm{C}(\mathrm{O}), 4-\mathrm{ClC}_{6} \mathrm{H}_{4} \mathrm{C}(\mathrm{O}), \mathrm{Ts}, \mathrm{P}(\mathrm{O}) \mathrm{Ph}_{2}, \mathrm{CO}_{2} \mathrm{Me}$, TMS

$\mathrm{X}=\mathrm{I}, \mathrm{Br}, \mathrm{Cl}$

Scheme 94. 


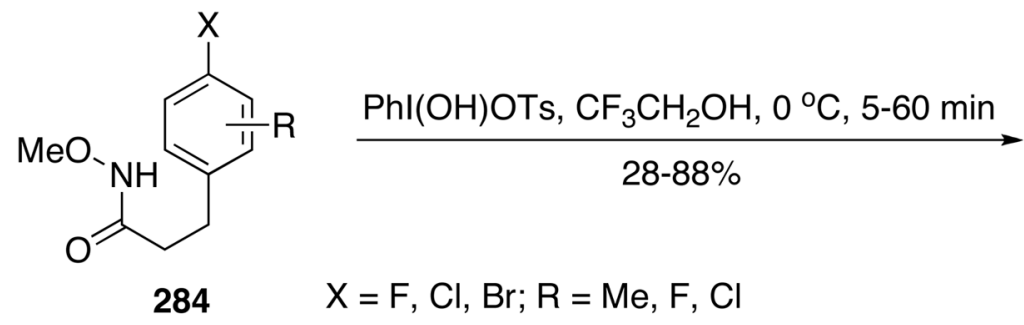

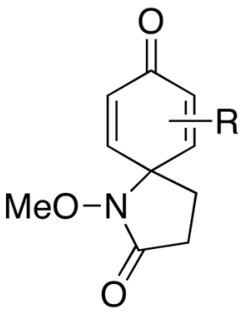

285

Scheme 95. 


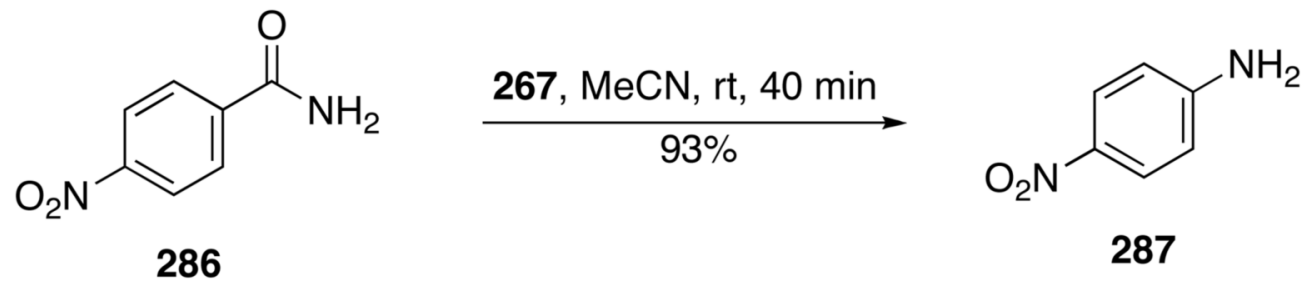

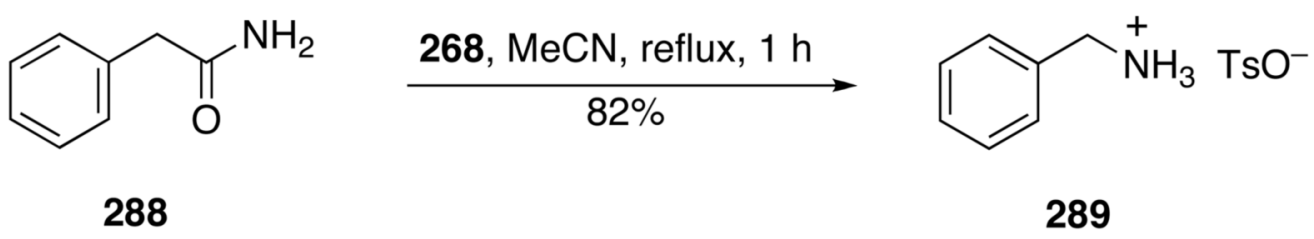

Scheme 96. 


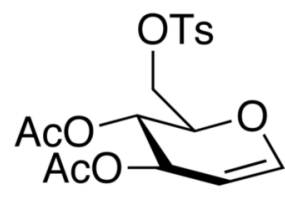

290
Phl(OH)OTs, mol. sieves $4 \AA$ $\mathrm{MeCN}, 0$ to $28^{\circ} \mathrm{C}, 2 \mathrm{~h}$

$45 \%$<smiles>CC(=O)OC[C@H]1OC=CC(=O)C1=O</smiles>

291

Scheme 97. 


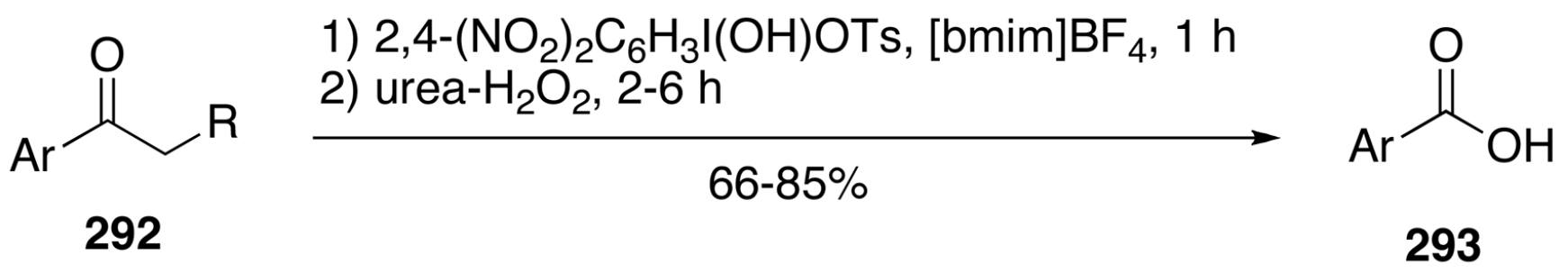

$$
\begin{aligned}
& \mathrm{Ar}=\mathrm{Ph}, 4-\mathrm{MeC}_{6} \mathrm{H}_{4}, 4-\mathrm{BrC}_{6} \mathrm{H}_{4}, 4-\mathrm{ClC}_{6} \mathrm{H}_{4}, 4-\mathrm{FC}_{6} \mathrm{H}_{4}, 4-\mathrm{NO}_{2} \mathrm{C}_{6} \mathrm{H}_{4} \\
& \mathrm{R}=\mathrm{Me}, \mathrm{Pr}, \mathrm{Bu}
\end{aligned}
$$

Scheme 98. 
<smiles>[R]C1=C([AsH2])CCC([R])C1</smiles>

294

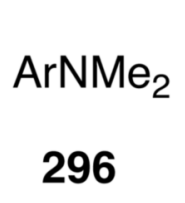

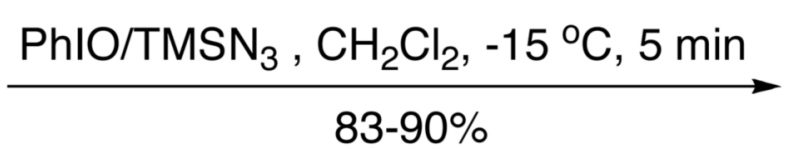

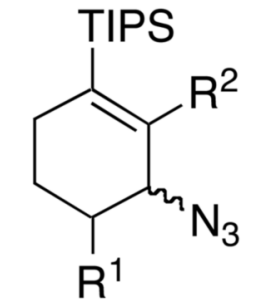

295

$\mathrm{Ar}=\mathrm{Ph}$, 4-pyridyl, 3-MeOC $\mathrm{H}_{6} \mathrm{H}_{4}$, Tol, Mes, etc.

Scheme 99. 

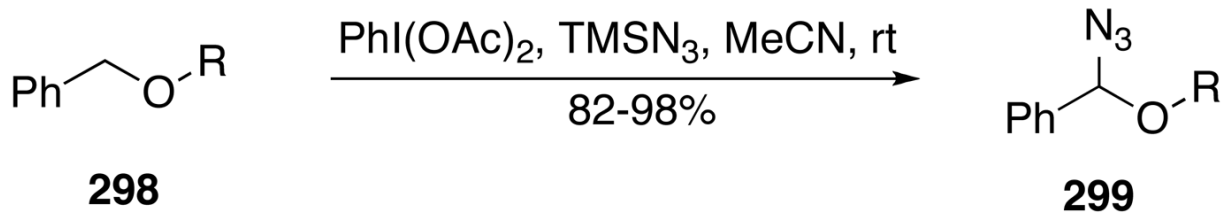

$\mathrm{R}=\mathrm{Me}, \mathrm{CH}_{2} \mathrm{Ph}, \mathrm{CH}_{2} \mathrm{CH}_{2} \mathrm{CH}_{2} \mathrm{OTMS}$, etc.

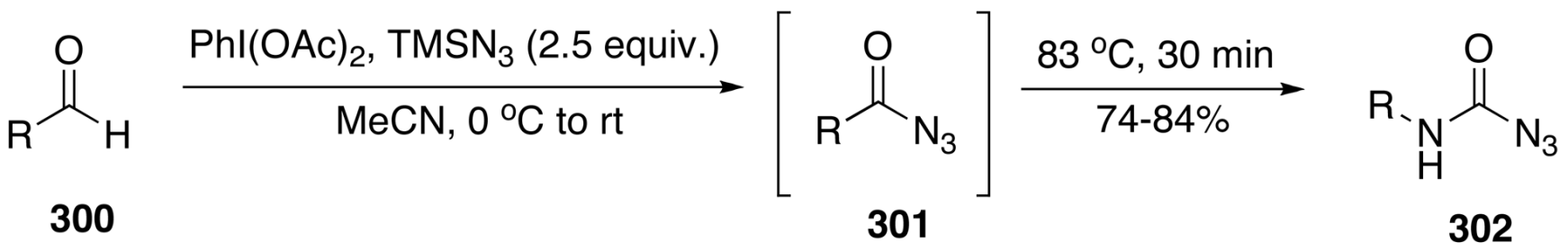

$\mathrm{R}=\mathrm{C}_{6} \mathrm{H}_{13}, \mathrm{PhCH}_{2} \mathrm{CH}_{2}, \mathrm{Ph}, 4-\mathrm{CH}_{3} \mathrm{C}_{6} \mathrm{H}_{4}, 4-\mathrm{MeOC}_{6} \mathrm{H}_{4}$, etc.

Scheme 100. 


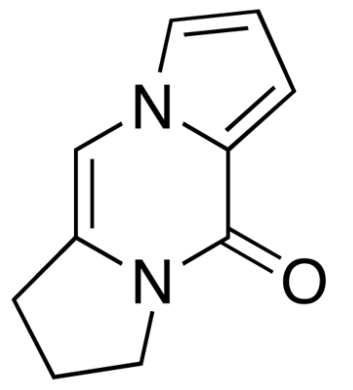

$\mathrm{Phl}(\mathrm{OAc})_{2}, \mathrm{TMSN}_{3}, \mathrm{Et}_{4} \mathrm{NI}$ $\mathrm{CH}_{2} \mathrm{Cl}_{2},-30^{\circ} \mathrm{C}$ to rt $41 \%$

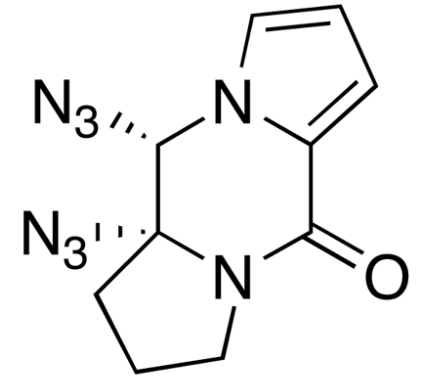

304

Scheme 101. 


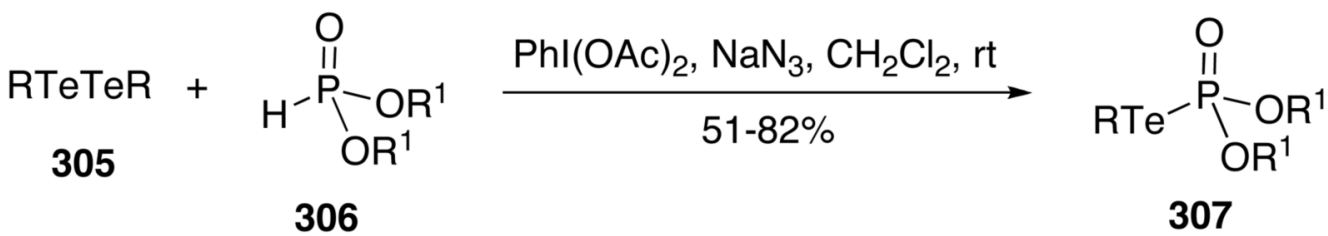

$\mathrm{R}=\mathrm{Ph}, \mathrm{Bu}, 4-\mathrm{ClC}_{6} \mathrm{H}_{4}, \alpha-\mathrm{C}_{10} \mathrm{H}_{7} ; \mathrm{R}^{1}=\mathrm{Me}, \mathrm{Et}, \mathrm{Pr}, \mathrm{Pr}^{\mathrm{i}}, \mathrm{Bu}, \mathrm{Ph}$

Scheme 102. 
<smiles>[R]C1=CC(=O)C([R7])=C([R])C1=O</smiles>

$\mathrm{R}^{1} / \mathrm{R}^{2}=\mathrm{H} / \mathrm{H}, \mathrm{Me} / \mathrm{Me}, \mathrm{Bu}^{\mathrm{t}} / \mathrm{H}, \mathrm{Cl} / \mathrm{H}$

Scheme 103. 

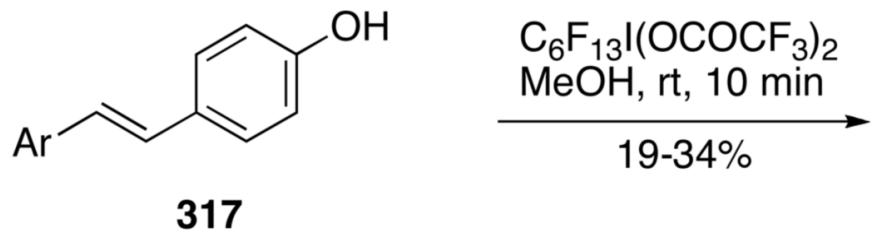

$$
\mathrm{Ar}=\mathrm{Ph}, 3-\mathrm{MeOC}_{6} \mathrm{H}_{4}, 3-\mathrm{FC}_{6} \mathrm{H}_{4}, 4-\mathrm{FC}_{6} \mathrm{H}_{4}
$$<smiles>COC(Br)C(OC)c1ccc(O)cc1</smiles>

Scheme 104. 


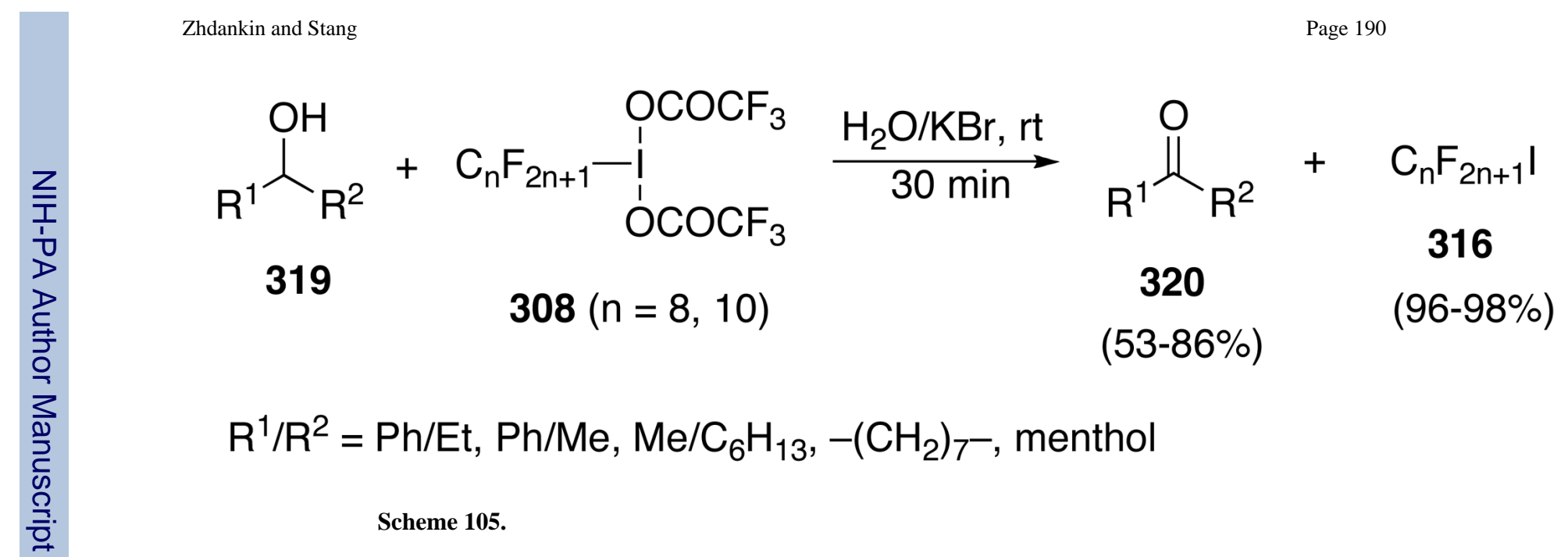


<smiles>COC(=O)C(P)NC(=O)c1ccccc1I</smiles>

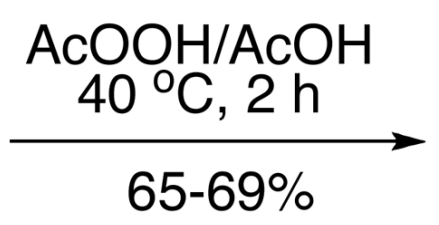<smiles>[R]C(C(=O)OC)N1C(=O)c2ccccc2I1OC(C)C</smiles>

\section{$326 \mathrm{R}=\mathrm{Me}$ or $\mathrm{Pr}^{\mathrm{i}}$}

Scheme 106. 


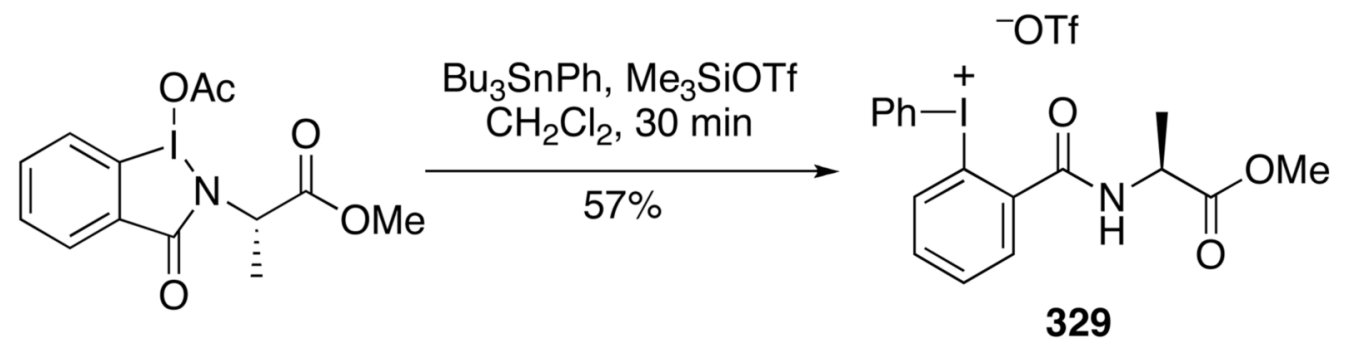

328

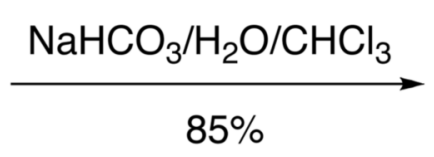<smiles>COC(=O)[C@H](C)N1C(=O)c2ccccc2I1c1ccccc1</smiles>

330

Scheme 107. 


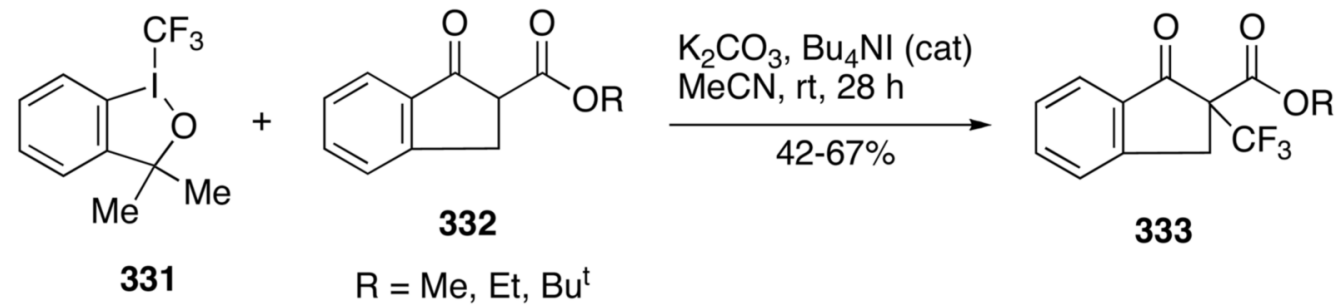

Scheme 108. 


$$
\begin{gathered}
\mathrm{C}_{\mathrm{n}} \mathrm{F}_{2 \mathrm{n}+1} \mathrm{CH}_{2}{\underset{\mathrm{OCOCF}}{\mathrm{O}}}_{\mathbf{O} \mathrm{n}=1,7,9}^{\mathrm{OCOCF}_{3}}+\mathrm{HN}\left(\mathrm{SO}_{2} \mathrm{CF}_{3}\right)_{2} \frac{\mathrm{PhH}, \mathrm{rt}, 18-22 \mathrm{~h}}{85-90 \%} \\
\mathrm{C}_{\mathrm{n}} \mathrm{F}_{2 \mathrm{n}+1} \mathrm{CH}_{2}-\mathrm{I}^{+} \\
\left(\mathrm{CF}_{3} \mathrm{SO}_{2}\right)_{2} \mathrm{~N}^{-} \\
\mathbf{3 3 7}
\end{gathered}
$$

Scheme 109. 
$\mathrm{HO}$

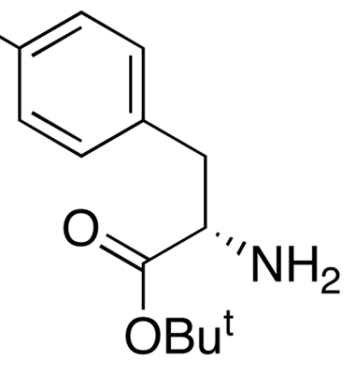

338 $337(n=7)$, collidine $\mathrm{CH}_{2} \mathrm{Cl}_{2}, \mathrm{rt}, 10 \mathrm{~min}$ quantitative

Scheme 110.

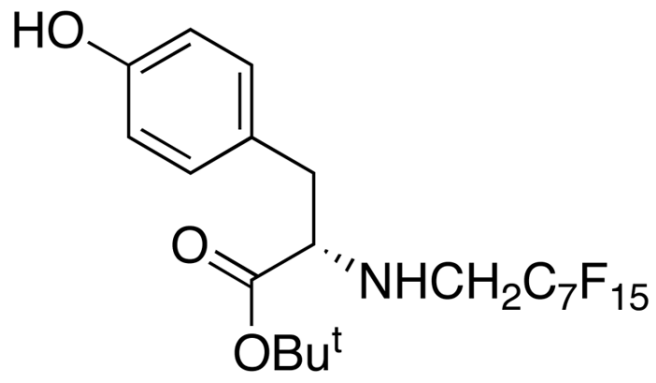

339 


$$
\begin{aligned}
& \text { 1. } \mathrm{AcOH}, \mathrm{rt} \\
& \begin{array}{cccc}
\operatorname{Arl}(\mathrm{OAc})_{2}+\left(4-\mathrm{MeOC}_{6} \mathrm{H}_{4}\right)_{4} \mathrm{~B}^{-} \mathrm{Na}^{+} & \stackrel{2 . \mathrm{NaBF}_{4}, \mathrm{H}_{2} \mathrm{O}}{\longrightarrow} & 4-\mathrm{MeOC}_{6} \mathrm{H}_{4} \mathrm{IAr}^{-}-\mathrm{BF}_{4} \\
\mathrm{Ar}=\mathrm{Ph} \text {, Tol } & \mathbf{3 4 0} & \mathbf{3 4 3 \%} & \mathbf{3 4 1}
\end{array} \\
& \text { 1. } \mathrm{BF}_{3} \cdot \mathrm{Et}_{2} \mathrm{O}, \mathrm{CH}_{2} \mathrm{Cl}_{2} \\
& \mathrm{Phl}(\mathrm{OAC})_{2}+\underset{\mathbf{3 4 2}}{\operatorname{ArB}(\mathrm{OH})_{2}} \stackrel{2 . \mathrm{NaBF}_{4}, \mathrm{H}_{2} \mathrm{O}}{73-83 \%} \underset{\mathbf{3 4 3}}{\stackrel{+}{\operatorname{PhIAr}}-\mathrm{BF}_{4}} \\
& \mathrm{Ar}=\mathrm{Ph}, 4-\mathrm{FC}_{6} \mathrm{H}_{4}, 4-\mathrm{ClC}_{6} \mathrm{H}_{4}, 4-\mathrm{MeOC}_{6} \mathrm{H}_{4} \text {, Tol }
\end{aligned}
$$




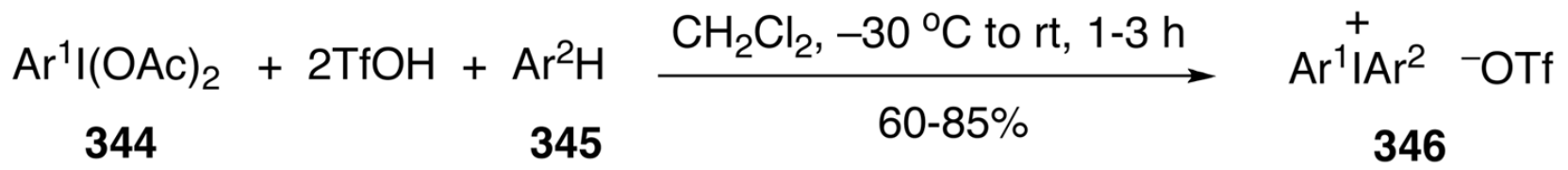

$\mathrm{Ar}^{1}=\mathrm{Ph}, 2-\mathrm{MeC}_{6} \mathrm{H}_{4}, 3-\mathrm{MeC}_{6} \mathrm{H}_{4}, 2,4,6-\mathrm{Me}_{3} \mathrm{C}_{6} \mathrm{H}_{2}, 3,5-\mathrm{Me}_{2} \mathrm{C}_{6} \mathrm{H}_{3}, 3-\mathrm{MeOC}_{6} \mathrm{H}_{4}$ $\mathrm{Ar}^{2}=4-\mathrm{MeC}_{6} \mathrm{H}_{4}, 4-\mathrm{MeOC}_{6} \mathrm{H}_{4}, 2,4,6-\mathrm{Me}_{3} \mathrm{C}_{6} \mathrm{H}_{2}, 4-\mathrm{Bu}^{\mathrm{t}} \mathrm{CH}_{2} \mathrm{C}_{6} \mathrm{H}_{4}$

Scheme 112. 


$$
\begin{array}{ll}
\text { ArH }+\mathrm{I}_{2} & \frac{\text { 1. } \mathrm{K}_{2} \mathrm{~S}_{2} \mathrm{O}_{8}, \mathrm{CF}_{3} \mathrm{CO}_{2} \mathrm{H}, 40{ }^{\circ} \mathrm{C}, 72 \mathrm{~h}}{11-69 \%} \\
\mathrm{Ar}=4-\mathrm{ClC}_{6} \mathrm{H}_{4}, 4-\mathrm{BrC}_{6} \mathrm{H}_{4}, 4-\mathrm{FC}_{6} \mathrm{H}_{4}, 4-\mathrm{IC}_{6} \mathrm{H}_{4}, 4-\mathrm{MeC}_{6} \mathrm{H}_{4}, 4-\mathrm{Bu}^{\mathrm{t}} \mathrm{C}_{6} \mathrm{H}_{4}
\end{array}
$$

Scheme 113. 


$$
\begin{aligned}
& m \mathrm{CPBA}, \mathrm{TfOH}, \mathrm{CH}_{2} \mathrm{Cl}_{2}
\end{aligned}
$$

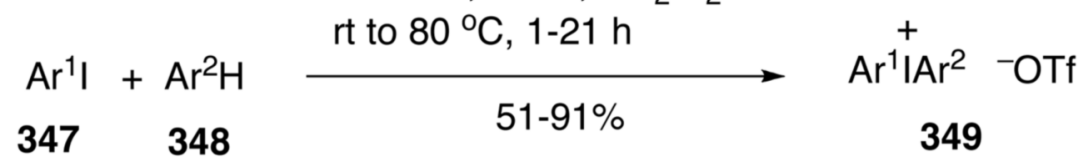

$$
\begin{aligned}
& \mathrm{Ar}^{1}=\mathrm{Ph}, 4-\mathrm{ClC}_{6} \mathrm{H}_{4}, 4-\mathrm{BrC}_{6} \mathrm{H}_{4}, 2-\mathrm{MeC}_{6} \mathrm{H}_{4}, 4-\mathrm{MeC}_{6} \mathrm{H}_{4}, 4-\mathrm{Bu}^{\mathrm{t}} \mathrm{C}_{6} \mathrm{H}_{4}, 4-\mathrm{NO}_{2} \mathrm{C}_{6} \mathrm{H}_{4} \text {, } \\
& \text { 4- } \mathrm{CF}_{3} \mathrm{C}_{6} \mathrm{H}_{4}, 4-\mathrm{HO}_{2} \mathrm{CC}_{6} \mathrm{H}_{4}, 3-\mathrm{CF}_{3} \mathrm{C}_{6} \mathrm{H}_{4} \text {, 2-chloro-5-pyridinyl } \\
& \mathrm{Ar}^{2}=\mathrm{Ph}, 4-\mathrm{ClC}_{6} \mathrm{H}_{4}, 4-\mathrm{BrC}_{6} \mathrm{H}_{4}, 4-\mathrm{FC}_{6} \mathrm{H}_{4}, 4-\mathrm{MeOC}_{6} \mathrm{H}_{4}, 4-\mathrm{Bu}^{t} \mathrm{C}_{6} \mathrm{H}_{4}, 4-\mathrm{MeC}_{6} \mathrm{H}_{4} \text {, } \\
& \text { 2,4,6- }-\mathrm{Me}_{3} \mathrm{C}_{6} \mathrm{H}_{2}, 2,5-\mathrm{Me}_{2} \mathrm{C}_{6} \mathrm{H}_{3}, 2,5-\mathrm{Bu}_{2}^{\mathrm{t}} \mathrm{C}_{6} \mathrm{H}_{3}
\end{aligned}
$$

Scheme 114. 


$$
(\mathrm{NC})_{2} \stackrel{+}{\mathrm{I}}-\mathrm{OTf}+2 \mathrm{ArSnBu}_{3} \quad \frac{\mathrm{CH}_{2} \mathrm{Cl}_{2},-40 \text { to } 20^{\circ} \mathrm{C}}{41-75 \%} \operatorname{Ar}_{2} \stackrel{+}{\mathrm{I}}-\mathrm{OTf}
$$

$\mathrm{Ar}=\mathrm{Ph}, 3-\mathrm{MeOC}_{6} \mathrm{H}_{4}, 4-\mathrm{MeOC}_{6} \mathrm{H}_{4}$, 2-furyl, 2-thienyl, 4-pyrazolyl, etc.

Scheme 115. 
<smiles>CC[SeH]c1ccc([AsH]C(C)C)s1</smiles>

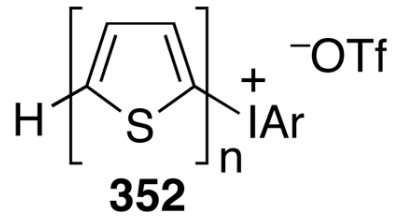

$$
\begin{aligned}
& \mathrm{Ar}=\mathrm{Ph}, \mathrm{n}=1 ; \mathrm{Ar}=\mathrm{Ph}, \mathrm{n}=2 \\
& \mathrm{Ar}=4-\mathrm{CF}_{3} \mathrm{C}_{6} \mathrm{H}_{4}, \mathrm{n}=2 ; \mathrm{Ar}=3-\mathrm{ClC}_{6} \mathrm{H}_{4}, \mathrm{n}=2
\end{aligned}
$$

Scheme 116. 

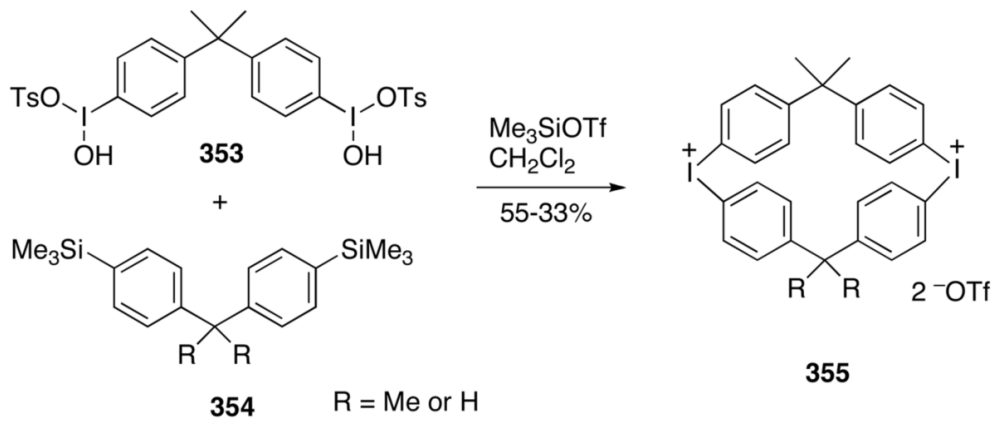

$354 \mathrm{R}=\mathrm{Me}$ or $\mathrm{H}$

355
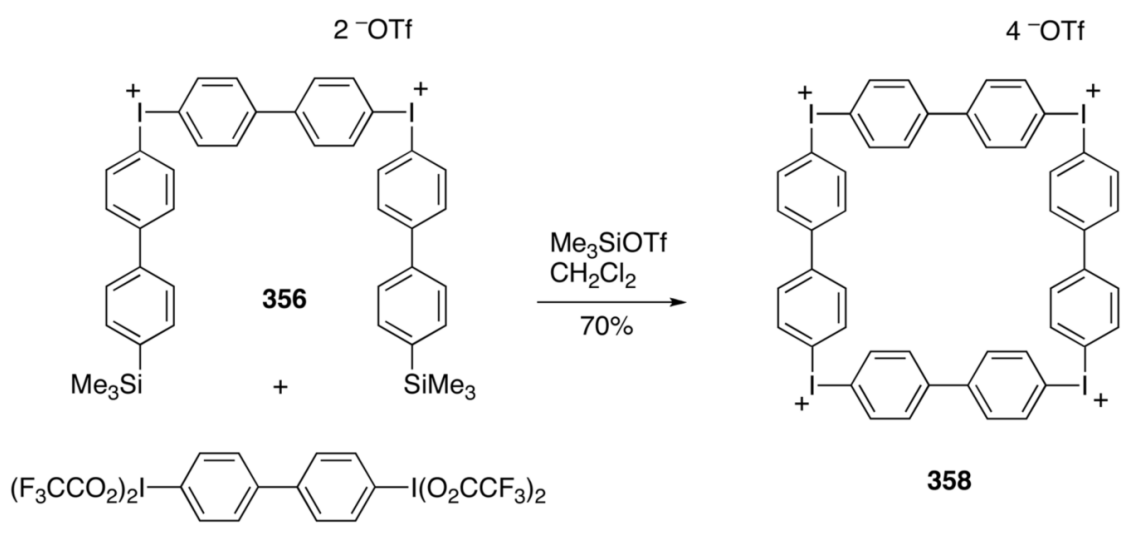

358

357
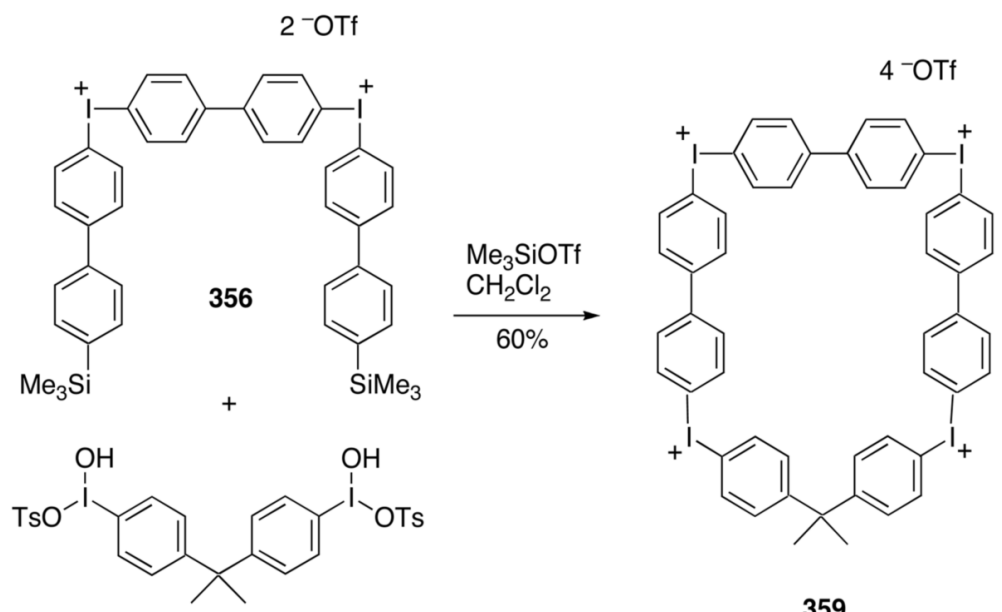

353

Scheme 117. 


$$
\underset{360}{\mathrm{ArLi}}+\underset{361}{\mathrm{Cl}} \mathrm{ICl}_{2} \stackrel{\mathrm{Et}_{2} \mathrm{O},-78^{\circ} \mathrm{C} \text { to rt }}{27-92 \%} \underset{362}{\mathrm{Ar}_{2}{ }^{+}-\mathrm{Cl}}
$$

$\mathrm{Ar}=\mathrm{Ph}, \mathrm{Tol}, 1$-naphthyl, 2-naphthyl, 2-thienyl, 2-furanyl, etc.

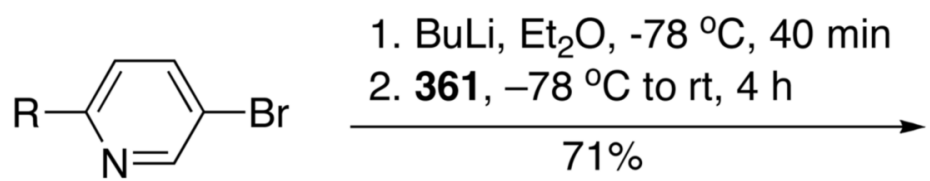

$363 \mathrm{R}=\mathrm{H}$ or $\mathrm{Cl}$<smiles>[R]c1ccc([C-](Cl)c2ccc(P)nc2)cn1</smiles>

Scheme 118. 
<smiles>COc1ccc(OC)c(CN(C(C)=O)c2cc([Al+]C)ccc2Oc2ccccc2)c1</smiles><smiles>COc1ccc(OC)c(CN(C(C)=O)c2cc(Br)ccc2Oc2ccccc2)c1</smiles>

Scheme 119. 
<smiles>O=C1CCC([N+](=O)[O-])CC1</smiles>

367

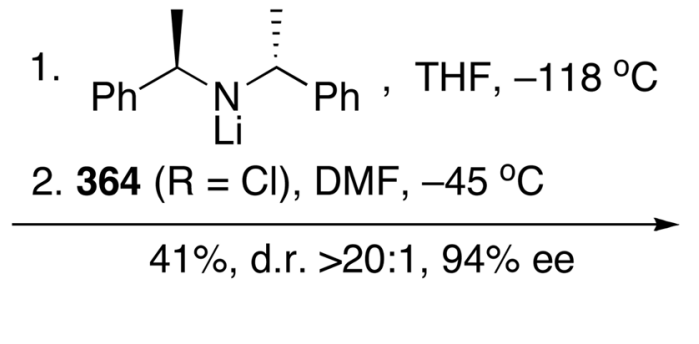

Scheme 120.<smiles></smiles>

368 

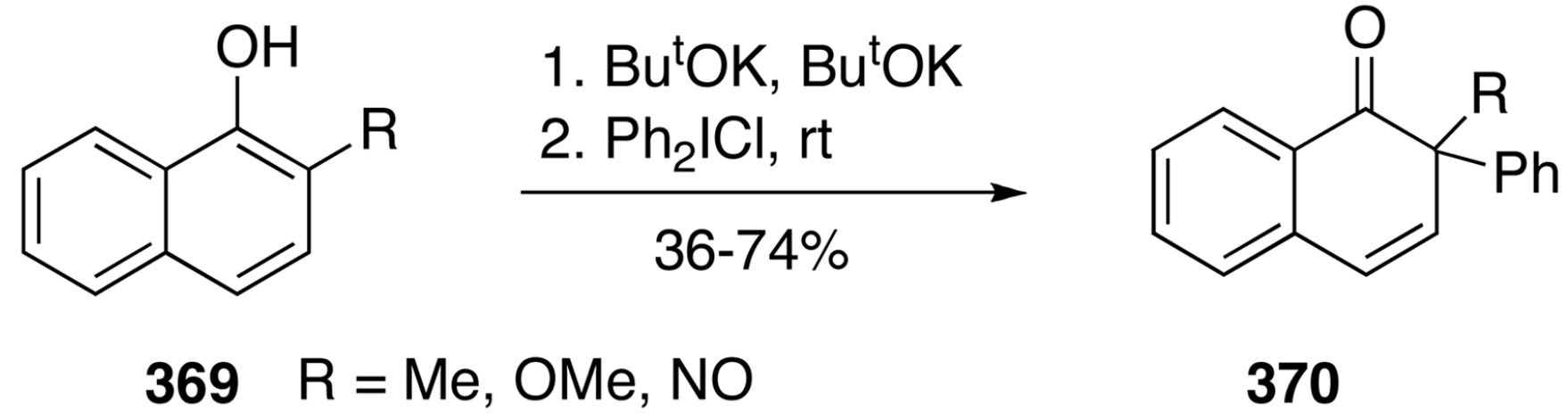

Scheme 121. 


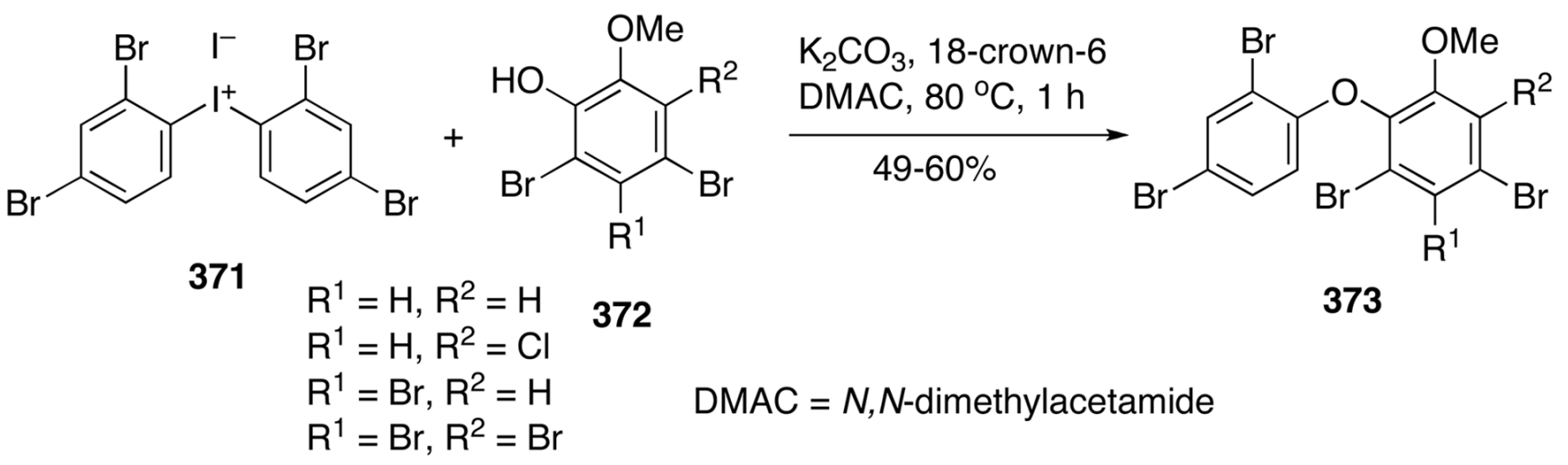

Scheme 122. 

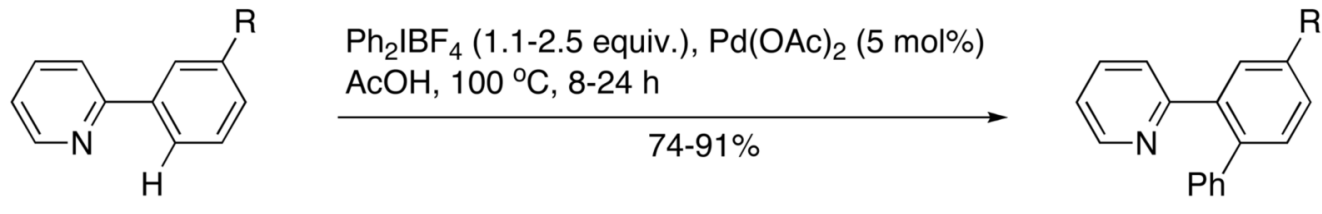

$374 \mathrm{R}=\mathrm{Me}, \mathrm{Ac}$, etc.

Scheme 123. 
<smiles>C[AsH2]c1ccccc1[In]O[Na]</smiles><smiles>c1ccccc1</smiles>
$\underset{68-97 \%}{\stackrel{\mathrm{RCO}_{2} \mathrm{H}}{\longrightarrow}} \begin{gathered}\mathrm{RCO}_{2} \mathrm{Ph} \\ 377\end{gathered}$ 376

$$
\mathrm{R}=\text { alkyl, aryl, alkenyl }
$$

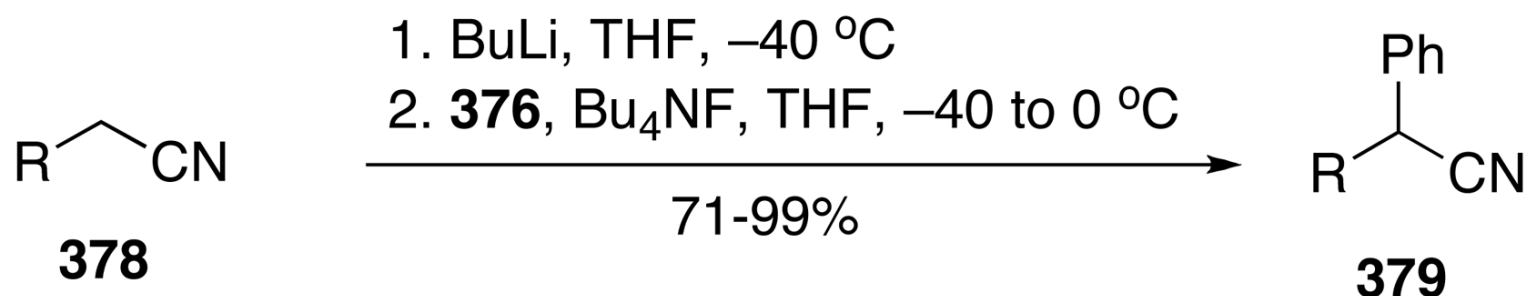

$\mathrm{R}=\mathrm{Me}, \mathrm{Et}, \mathrm{Pr}, \mathrm{Bu}, \mathrm{CN}, \mathrm{CH}_{2} \mathrm{CN}$, etc.<smiles>CC1=C([Al])C([Al])=C(C)S1=O</smiles>

$380 \quad \mathrm{Ar}=4-\mathrm{MeOC}_{6} \mathrm{H}_{4}$
376, $\mathrm{Bu}_{4} \mathrm{NF}, \mathrm{THF}, 0^{\circ} \mathrm{C}$ to rt

$$
55 \%
$$<smiles>Cc1c([Al])c(Br)c(C)c2ccccc12</smiles>

381

Scheme 124. 
<smiles>[R]C(=O)c1ccc(S(C)(C)C)c([P+]([O-])[O-])c1</smiles><smiles>[R]C(=O)c1ccccc1</smiles><smiles></smiles>

$382 \mathrm{R}=\mathrm{Ph}, \mathrm{Tol}, \mathrm{Bu}^{\mathrm{t}}, \mathrm{Me}$

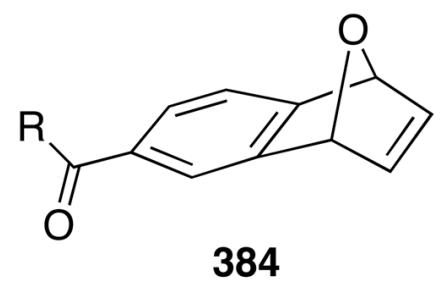

Scheme 125. 


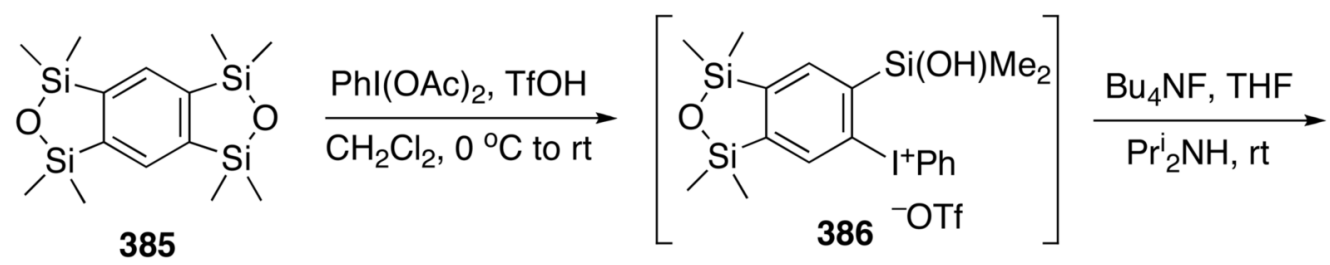<smiles>C[Si]1(C)O[Si](C)(C)c2ccccc21</smiles>

387
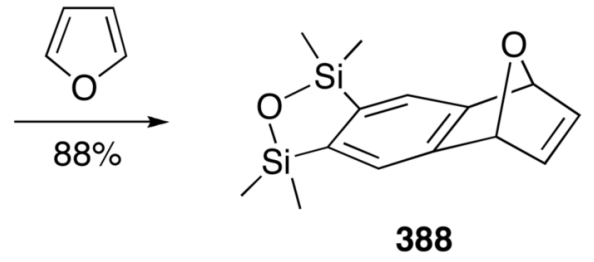

Scheme 126. 
1. $\mathrm{BF}_{3} \cdot \mathrm{Et}_{2} \mathrm{O}, \mathrm{CH}_{2} \mathrm{Cl}_{2}, \mathrm{O}^{\circ} \mathrm{C}$ to rt<smiles></smiles>

2. $\mathrm{NaBF}_{4} / \mathrm{H}_{2} \mathrm{O}$ $\mathrm{R}^{2} \quad \mathrm{TMS}$

$72-92 \%$

389

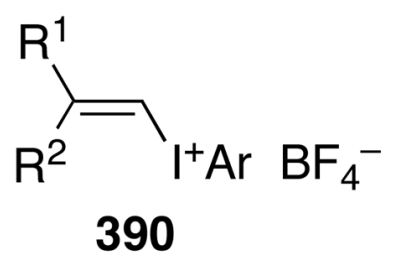

$\mathrm{R}^{1}=4-\mathrm{BrC}_{6} \mathrm{H}_{4} \mathrm{OCH}_{2}, \mathrm{PhCH}_{2} \mathrm{CH}_{2}, 4-\mathrm{ClC}_{6} \mathrm{H}_{4} \mathrm{OCH}_{2}, \mathrm{n}-\mathrm{C}_{8} \mathrm{H}_{17}$, etc. $\mathrm{R}^{2}=\mathrm{H}, \mathrm{Me} ; \mathrm{Ar}=\mathrm{Ph}, 2,4,6-\mathrm{Me}_{3} \mathrm{C}_{6} \mathrm{H}_{2}$, etc.

Scheme 127. 


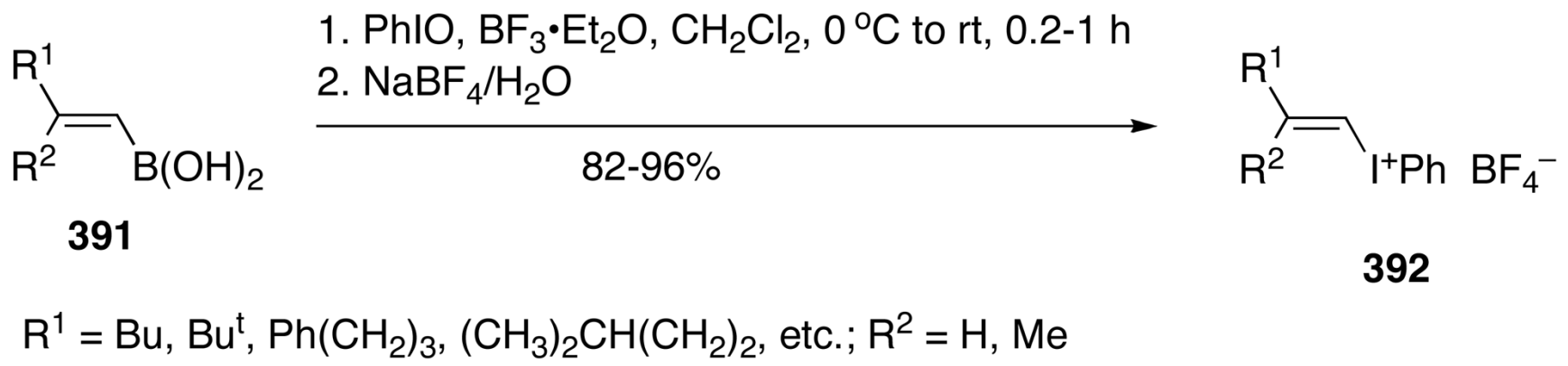

Scheme 128. 


$$
\begin{aligned}
& \mathrm{R}=+ \text { TollF}_{2} \frac{\text { 1. } \mathrm{BF}_{3} \cdot \mathrm{Et}_{2} \mathrm{O}, \mathrm{CH}_{2} \mathrm{Cl}_{2},-78^{\circ} \mathrm{C}, 5 \mathrm{~min}}{76-85 \%} \\
& \mathrm{R}=\mathrm{AcO}\left(\mathrm{CH}_{2}\right)_{9}, \mathrm{Cl}\left(\mathrm{CH}_{2}\right)_{9}, \mathrm{MeOOC}\left(\mathrm{CH}_{2}\right)_{8}, \mathrm{Bu}{ }^{\mathrm{t} C O}\left(\mathrm{CH}_{2}\right)_{8},\left(\text { cyclo }-\mathrm{C}_{6} \mathrm{H}_{11}\right) \mathrm{CH}_{2}
\end{aligned}
$$

Scheme 129. 


$$
\begin{aligned}
& \left.\mathrm{R}=\mathrm{I}_{394}=\mathrm{Ph} \mathrm{BF}_{4}^{-} \frac{20 \% \text { aq. } \mathrm{HF}, \mathrm{CHCl}_{3}, 60^{\circ} \mathrm{C}}{72-84 \%} \sum_{395}^{\mathrm{F}}\right\rangle_{\mathrm{I}^{+} \mathrm{Ph} \mathrm{BF}_{4}^{-}} \\
& \mathrm{R}=\mathrm{C}_{10} \mathrm{H}_{21}, \mathrm{Bu}^{\mathrm{t}},\left(\text { cyclo }-\mathrm{C}_{6} \mathrm{H}_{11}\right) \mathrm{CH}_{2}, \mathrm{Cl}\left(\mathrm{CH}_{2}\right)_{9}, \mathrm{Bu} \mathrm{CO}\left(\mathrm{CH}_{2}\right)_{8} \text {, PriOCO }\left(\mathrm{CH}_{2}\right)_{8}
\end{aligned}
$$

Scheme 130. 


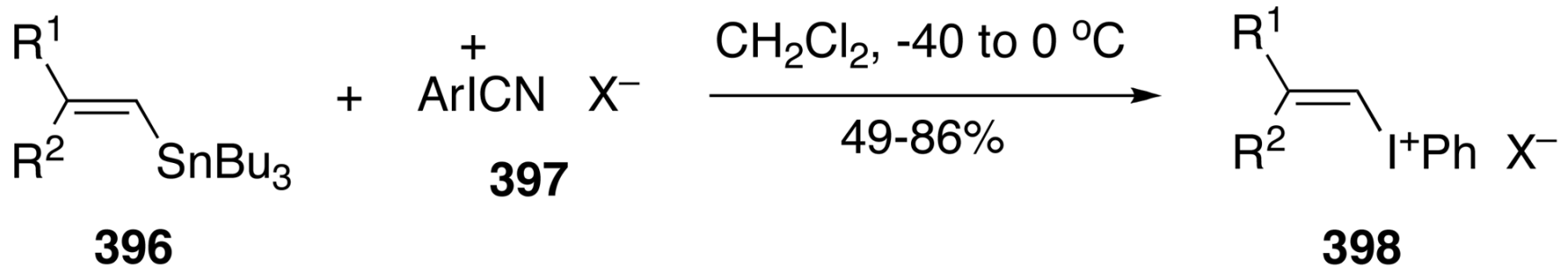

$\mathrm{R}^{1}=\mathrm{Me}, \mathrm{Et}, \mathrm{Bu}, \mathrm{Ph} ; \mathrm{R}^{2}=\mathrm{Me}, \mathrm{Et}, \mathrm{Bu}$

$\mathrm{Ar}=\mathrm{Ph}, 4-\mathrm{CF}_{3} \mathrm{C}_{6} \mathrm{H}_{4}, 3,5-\left(\mathrm{CF}_{3}\right)_{2} \mathrm{C}_{6} \mathrm{H}_{2} ; \mathrm{X}=\mathrm{OTf}$ or OTs

Scheme 131. 

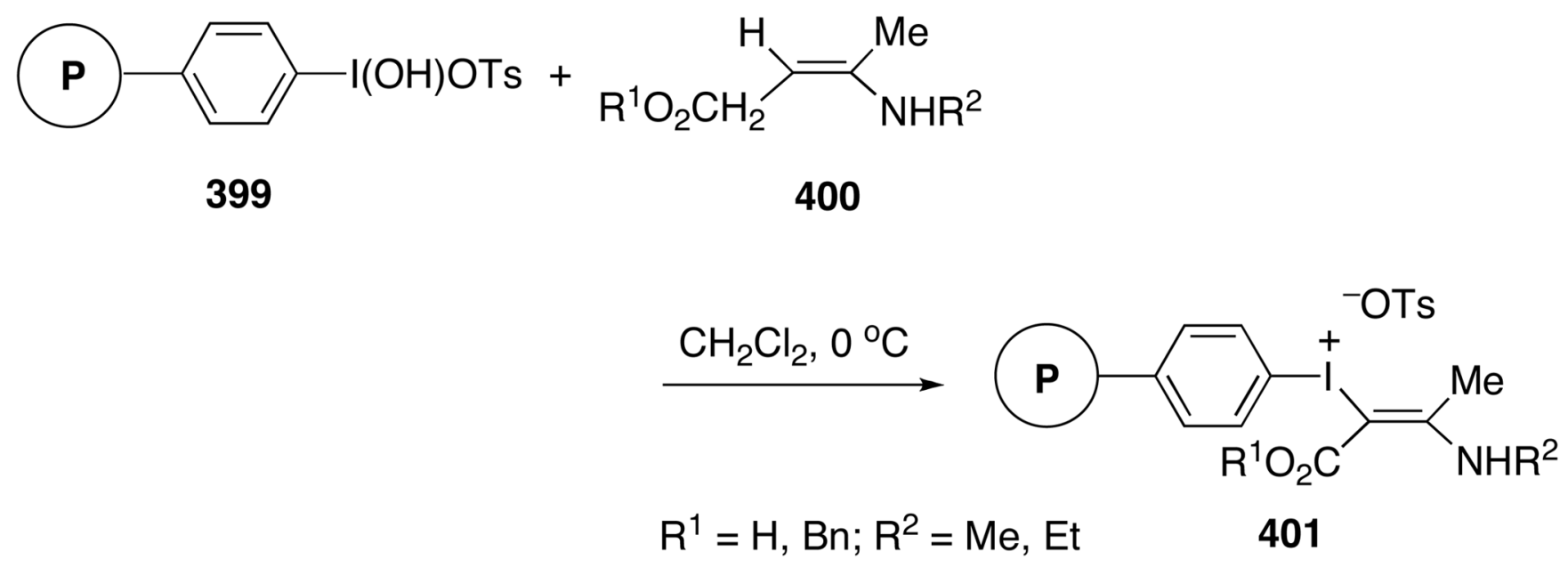

Scheme 132. 


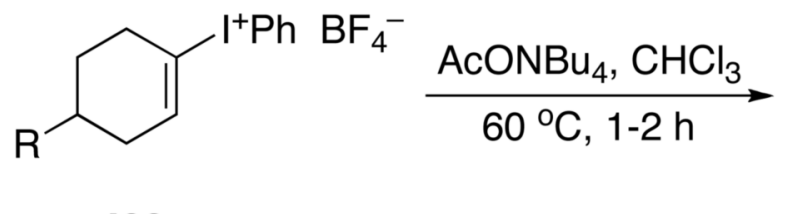

402

$\mathrm{R}=\mathrm{H}, \mathrm{Me}, \mathrm{Et}, \mathrm{Bu}^{\mathrm{t}}, \mathrm{Ph}, \mathrm{CN}$<smiles>CC(C)(C)O[R6](=O)(O)c1ccccc1</smiles><smiles>[R]C1CC#CCC1</smiles>
403<smiles>[R]C1CCc2c(c(-c3ccccc3)c(-c3ccccc3)c(-c3ccccc3)c2-c2ccccc2)C1</smiles>

Scheme 133. 


$$
\begin{aligned}
& \text { (P) } \\
& \begin{array}{l}
\mathrm{Nu}=\mathrm{KSCN}, \mathrm{HNEt}, \mathrm{Bu}_{4} \mathrm{NBr}, \mathrm{NaCN}, \mathrm{NaSO}_{2} \mathrm{Tol}, \text { morpholine, } \\
\mathrm{NaSC}(\mathrm{S}) \mathrm{N}\left(\mathrm{CH}_{3}\right) \mathrm{CH}_{2} \mathrm{Ph}, \mathrm{KSP}(\mathrm{O})(\mathrm{OEt})_{2}
\end{array}
\end{aligned}
$$

Scheme 134. 

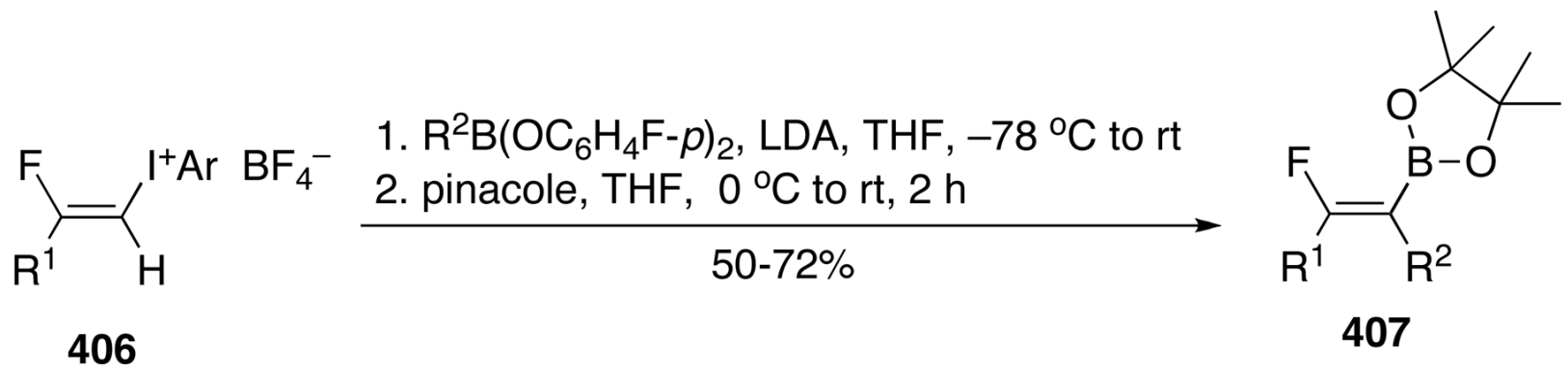

$\mathrm{R}^{1}=\mathrm{C}_{10} \mathrm{H}_{21}, \mathrm{AcO}\left(\mathrm{CH}_{2}\right)_{9}, \mathrm{BnO}\left(\mathrm{CH}_{2}\right)_{3}, \mathrm{BnO}_{2} \mathrm{C}\left(\mathrm{CH}_{2}\right)_{3}$, etc. $\mathrm{Ar}=\mathrm{Ph}$ or Tol; $\mathrm{R}^{2}=\mathrm{C}_{6} \mathrm{H}_{13}, \mathrm{BuCH}=\mathrm{CH}, \mathrm{Br}\left(\mathrm{CH}_{2}\right)_{3}$
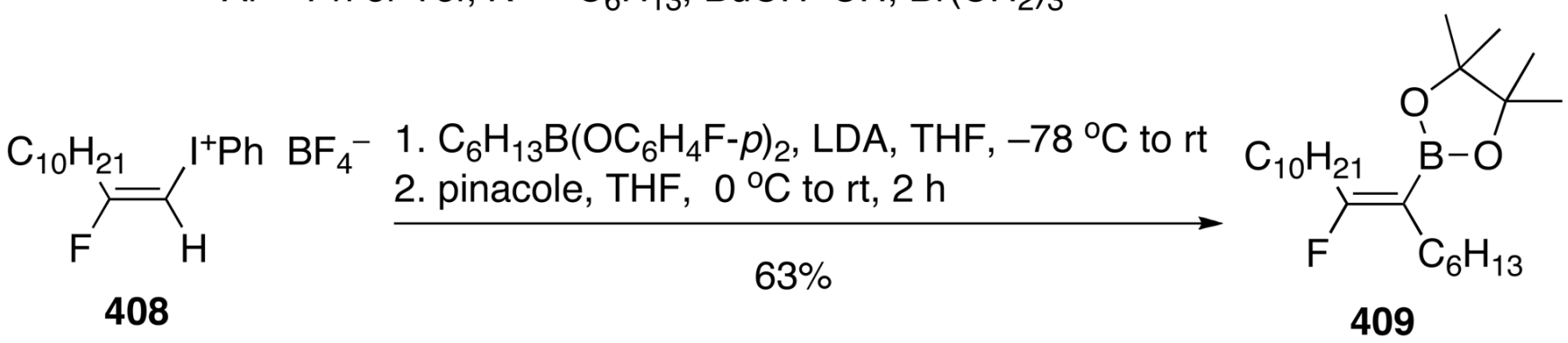

Scheme 135. 


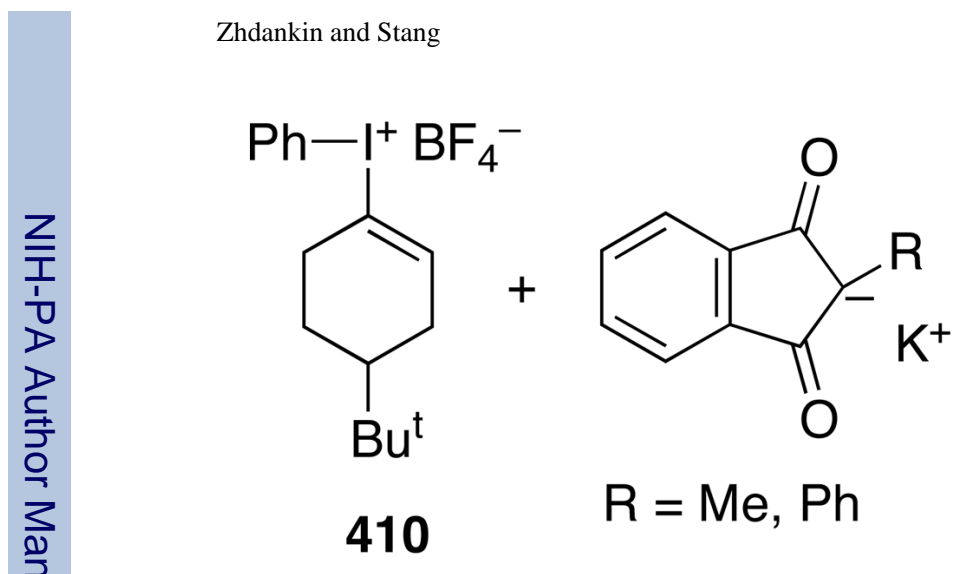

$\underset{86 \%}{\stackrel{\text { THF, rt, 1-4 h }}{\longrightarrow}}$

Scheme 136. 


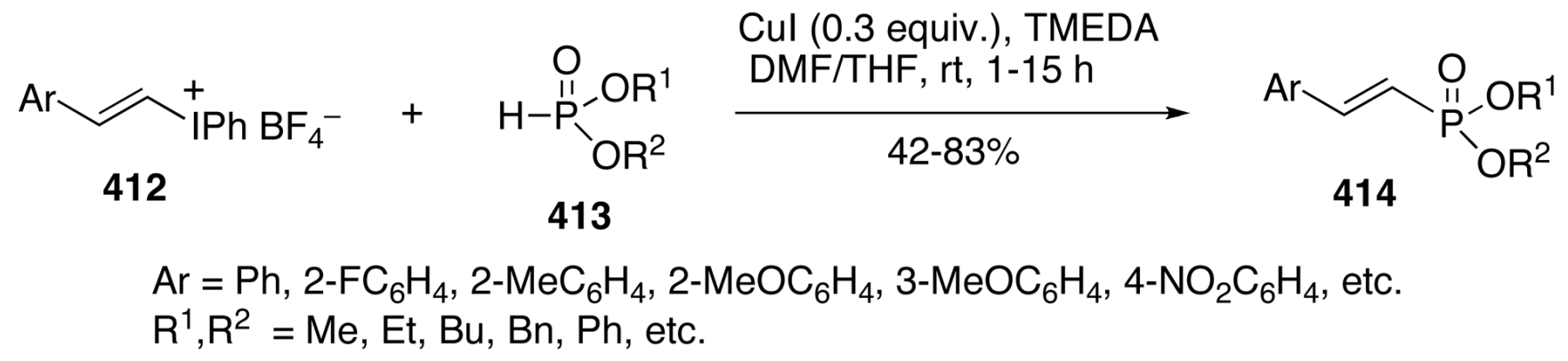

Scheme 137. 


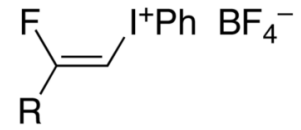

415

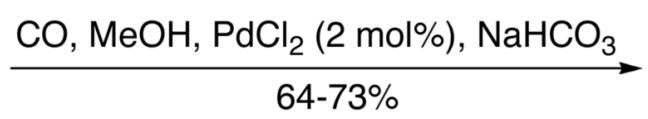

$64-73 \%$

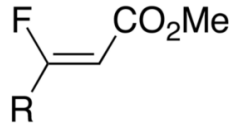

416

$$
\mathrm{R}=\mathrm{C}_{10} \mathrm{H}_{21},\left(\text { cyclo- } \mathrm{C}_{6} \mathrm{H}_{11}\right) \mathrm{CH}_{2}, \mathrm{Ph}, \mathrm{Cl}\left(\mathrm{CH}_{2}\right)_{9}, \mathrm{Pr}^{\mathrm{i}} \mathrm{O}_{2} \mathrm{C}\left(\mathrm{CH}_{2}\right)_{8}, \mathrm{Bu}{ }^{\mathrm{t}} \mathrm{CO}\left(\mathrm{CH}_{2}\right)_{8}
$$

Scheme 138. 

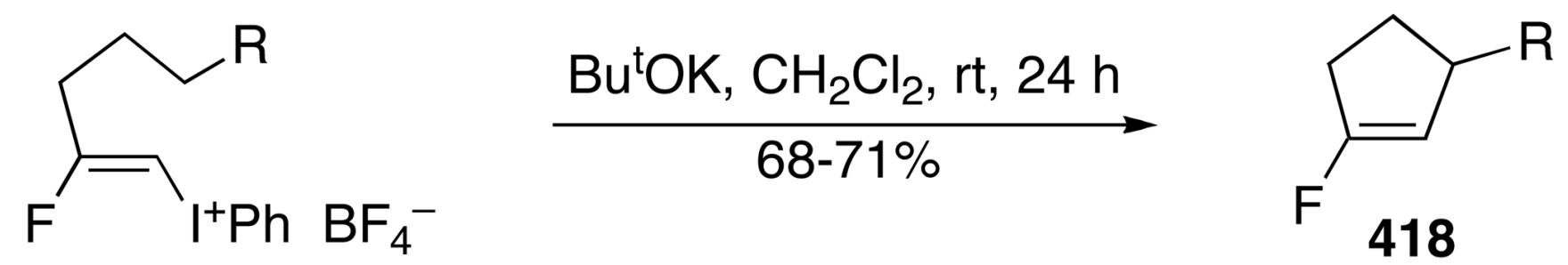

$417 \mathrm{R}=\mathrm{AcO}\left(\mathrm{CH}_{2}\right)_{6}, \mathrm{Cl}\left(\mathrm{CH}_{2}\right)_{6}, \mathrm{Bu}{ }^{\mathrm{t}} \mathrm{CO}\left(\mathrm{CH}_{2}\right)_{5}$, etc.

Scheme 139. 

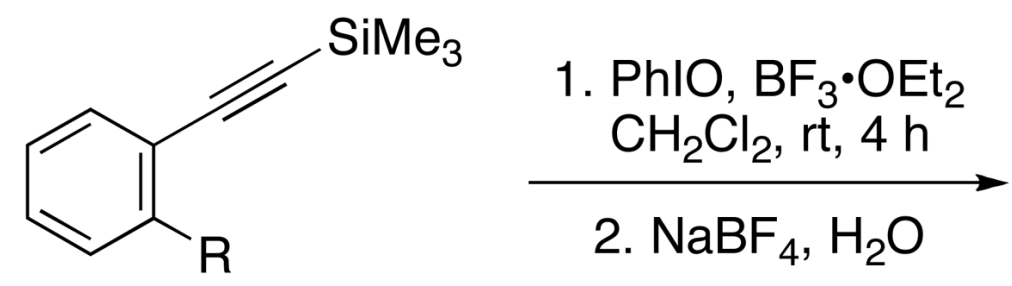

$419 \mathrm{R}=\mathrm{Me}, \mathrm{Et}, \mathrm{CH}_{2} \mathrm{OMe}$

Scheme 140.

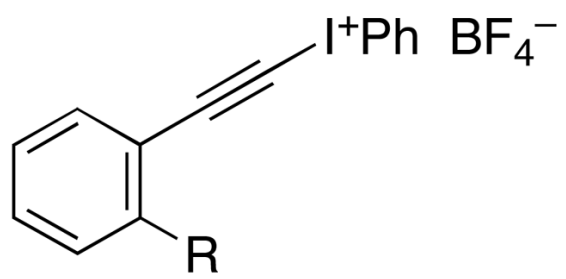

420 


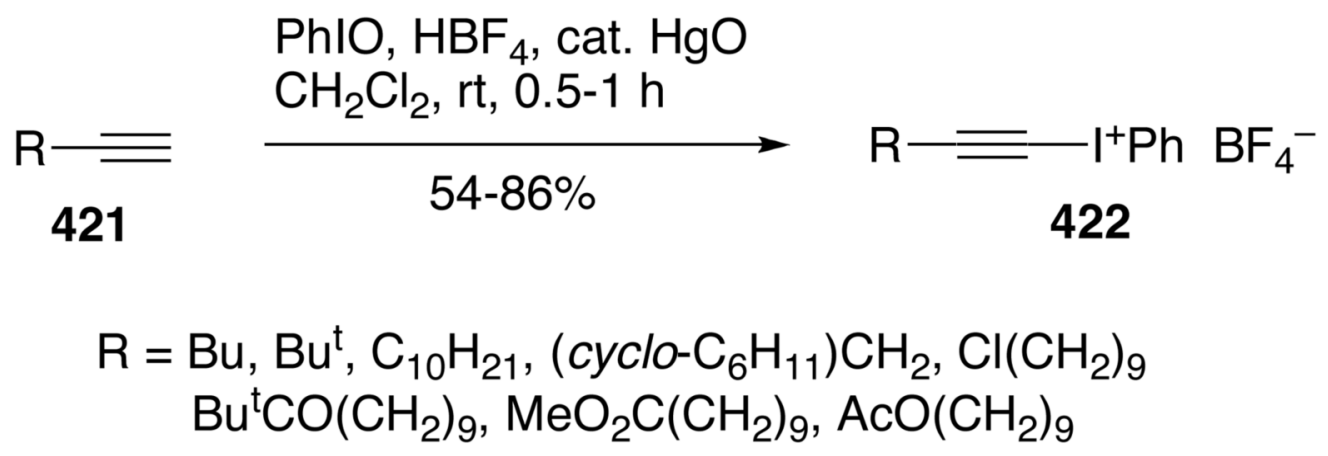

Scheme 141. 


$\mathrm{R} \underset{\mathbf{4 2 3}}{=} \mathrm{BF}_{3}{ }^{-} \mathrm{K}^{+} \stackrel{\mathrm{ArlF}_{2}, \mathrm{MeCN}, \mathrm{rt}, 15 \mathrm{~min}}{62-95 \%} \mathrm{R} \underset{\mathbf{4 2 4}}{=} \mathrm{I}^{+} \mathrm{Ar} \mathrm{BF}_{4}^{-}$
$\mathrm{R}=\mathrm{C}_{10} \mathrm{H}_{21}, \mathrm{BnOCH}_{2} ; \mathrm{Ar}=\mathrm{Tol}, \mathrm{Ph}, 4-\mathrm{ClC}_{6} \mathrm{H}_{4}$

Scheme 142. 

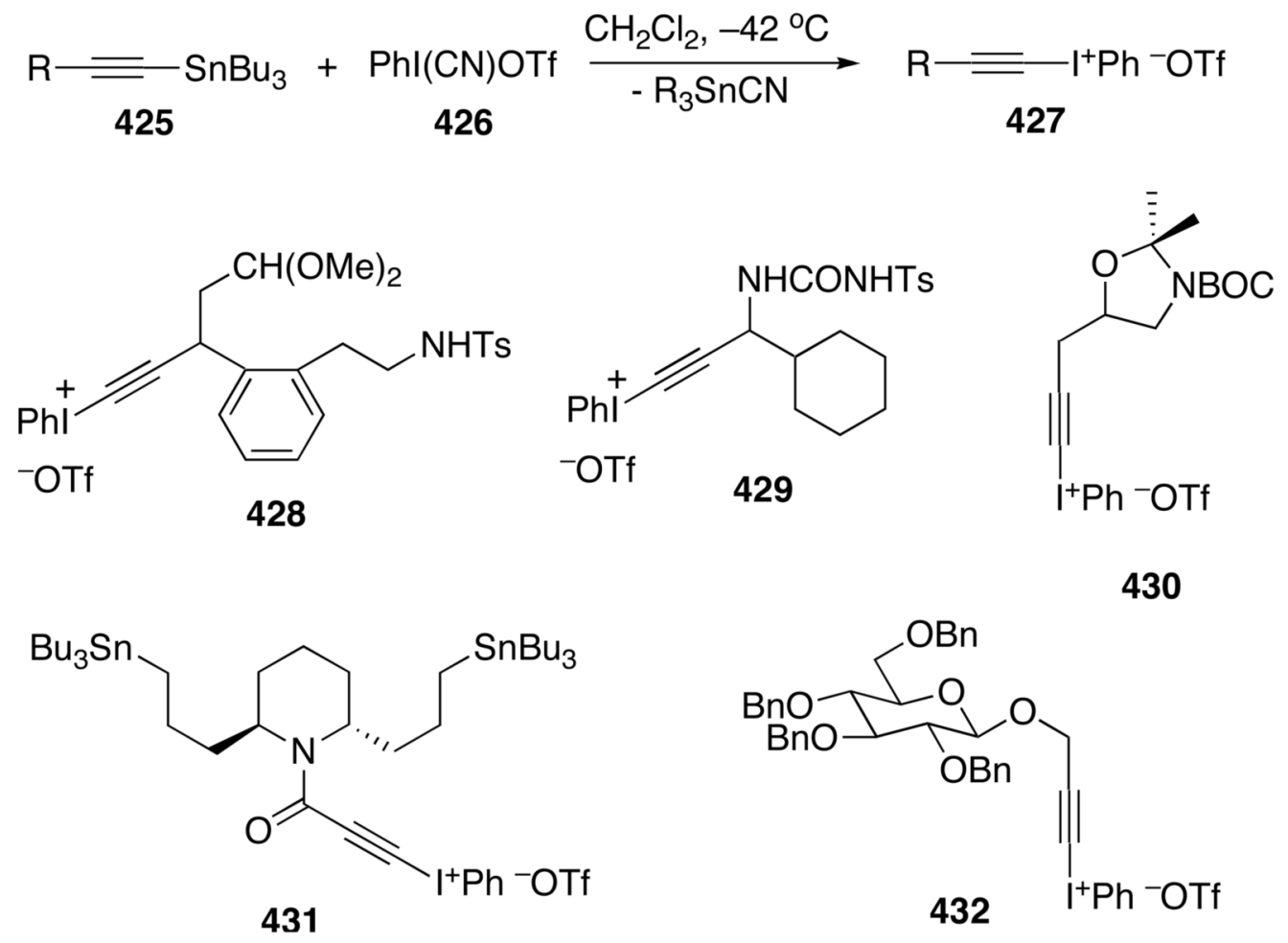

Scheme 143. 


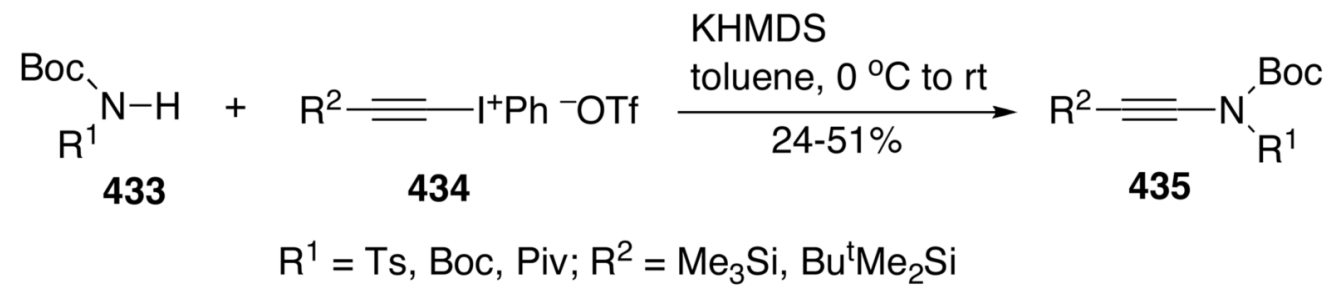

Scheme 144. 

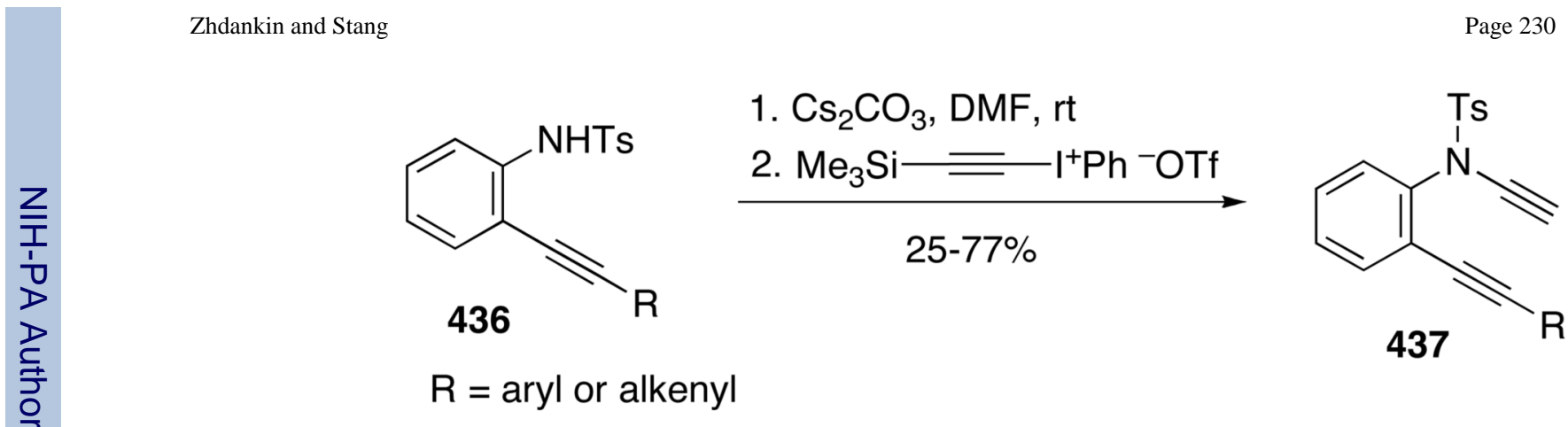

Scheme 145. 


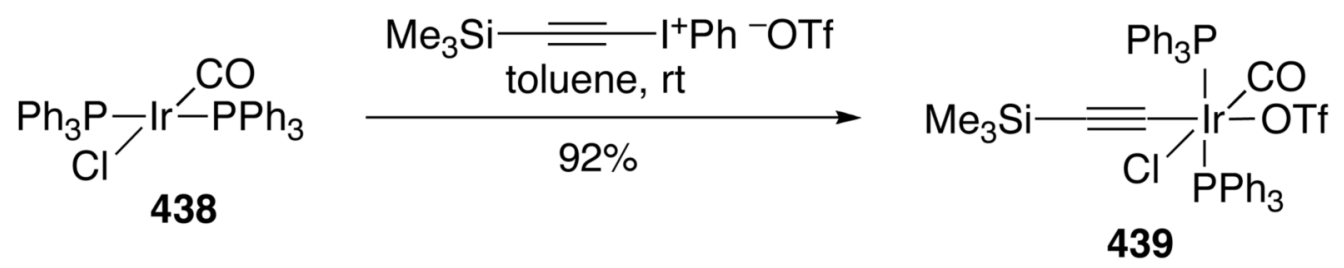

Scheme 146. 

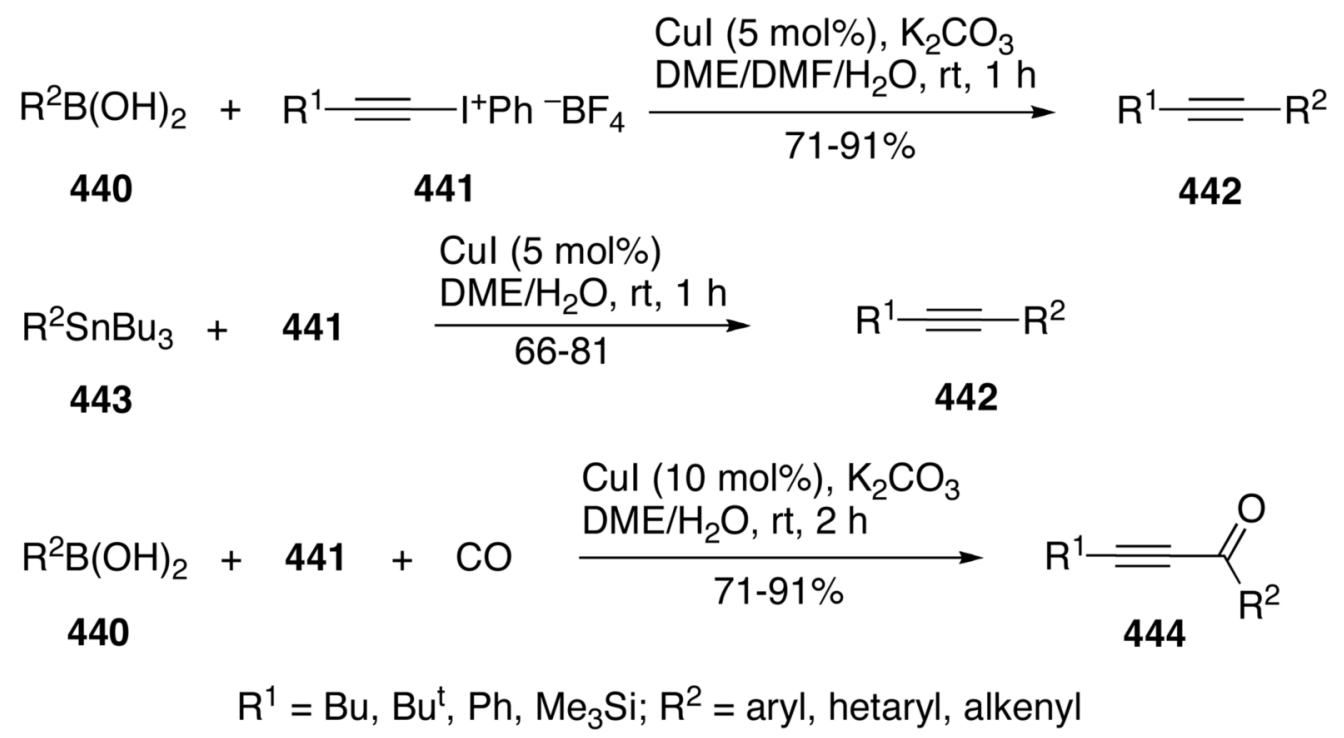

Scheme 147. 


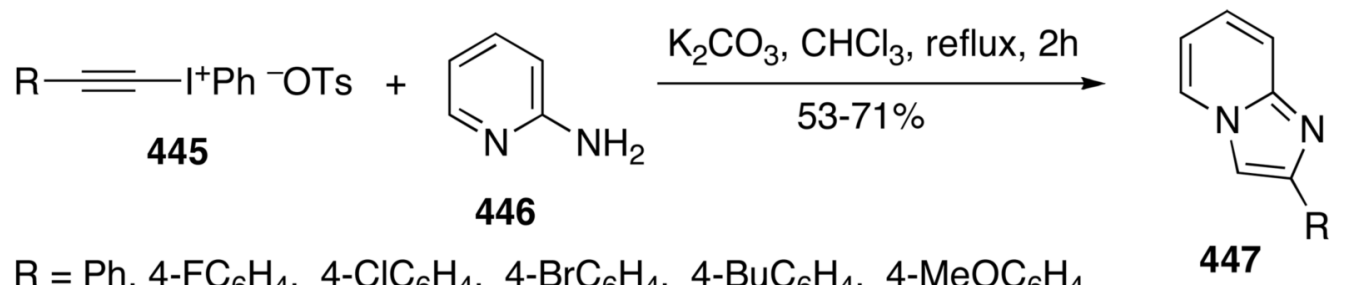

Scheme 148. 


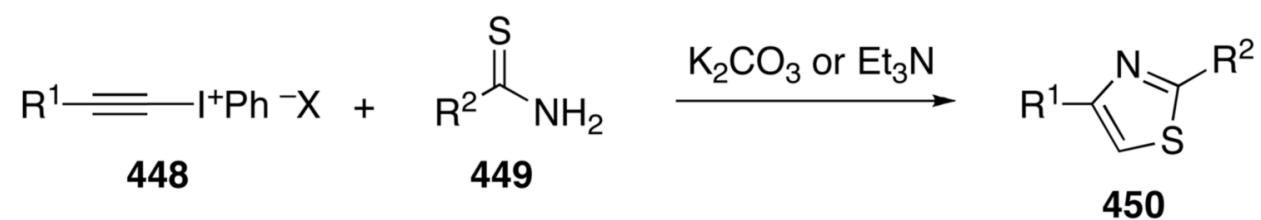<smiles>[R]C(=N)SC([R])=C[In+]</smiles>

451

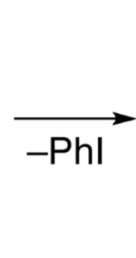<smiles>[R7]C(=C)SC([R])=N</smiles>

452

Scheme 149. 


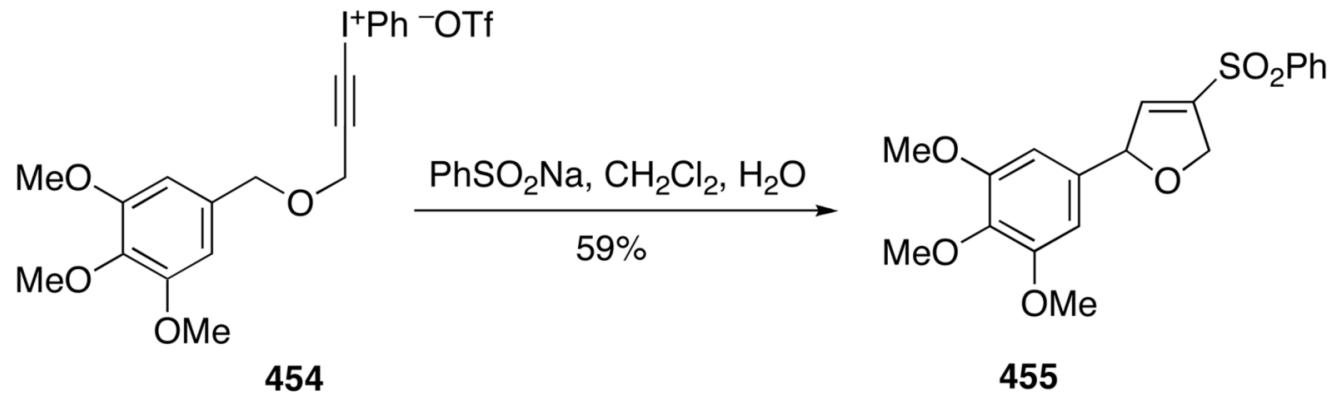

Scheme 150. 
<smiles>[R]N([CH])C(=O)N1C[C@H](CC#C[InH+])OC1=O</smiles>

$\underset{34 \%}{\stackrel{\mathrm{TolSO}_{2} \mathrm{Na}, \mathrm{DME}, 0^{\circ} \mathrm{C}}{\longrightarrow}}$

$456 \mathrm{R}=2-\mathrm{NO}_{2} \mathrm{C}_{6} \mathrm{H}_{4} \mathrm{CH}_{2}$<smiles>[R]N([Y19])C(=O)N1C(=O)O[C@@H]2CC(S(=O)(=O)[O-])=C[C@H]21</smiles>

457

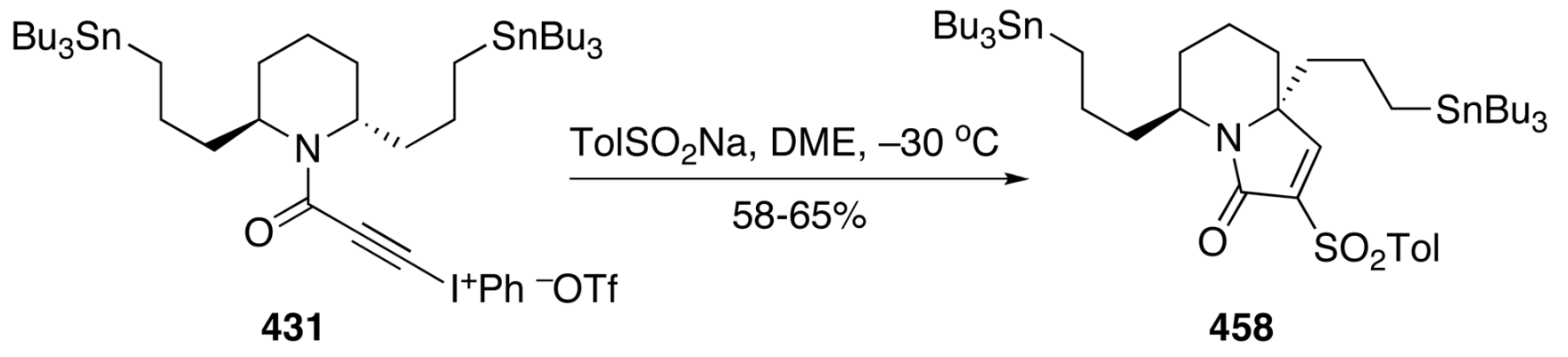

Scheme 151. 


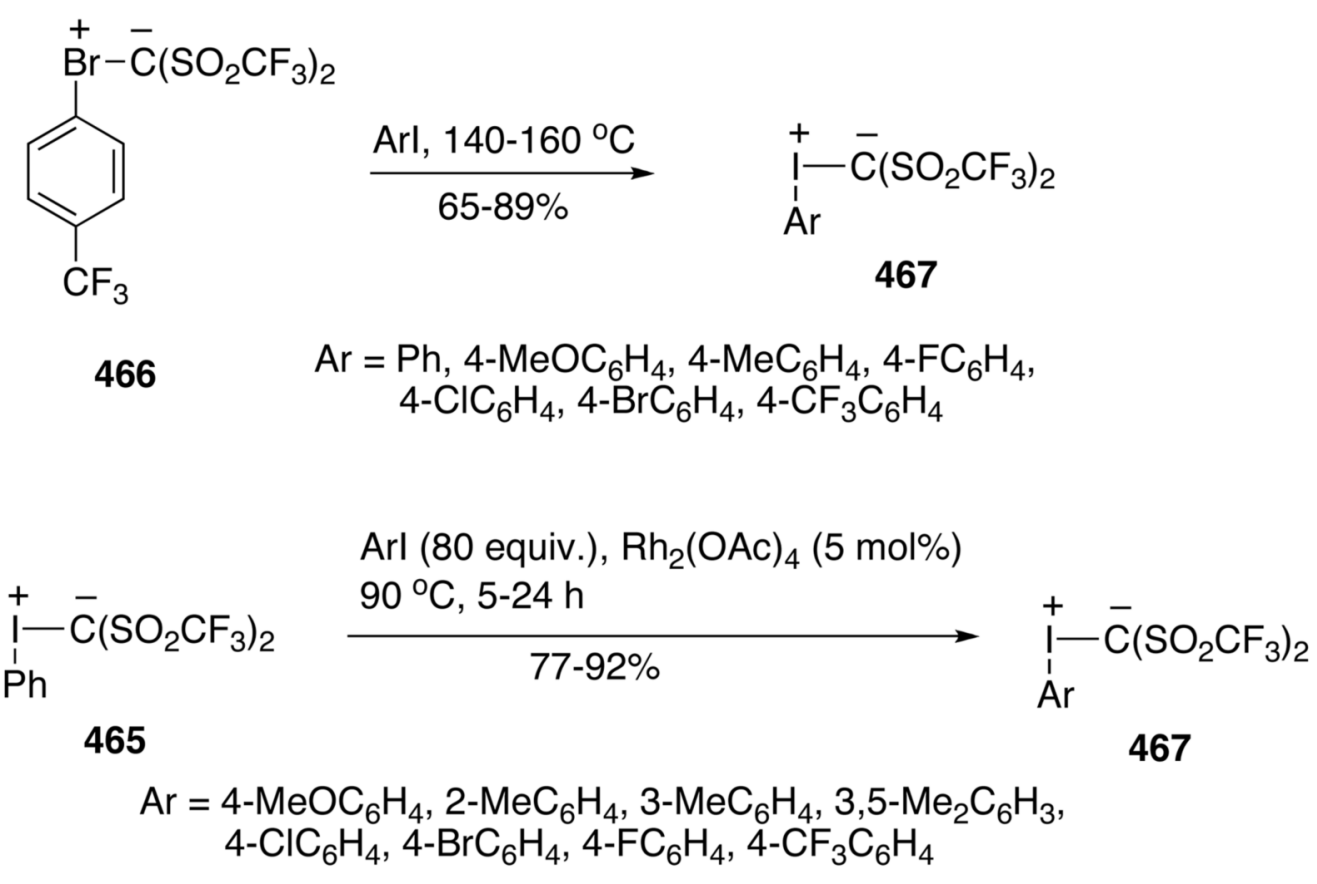

Scheme 152. 


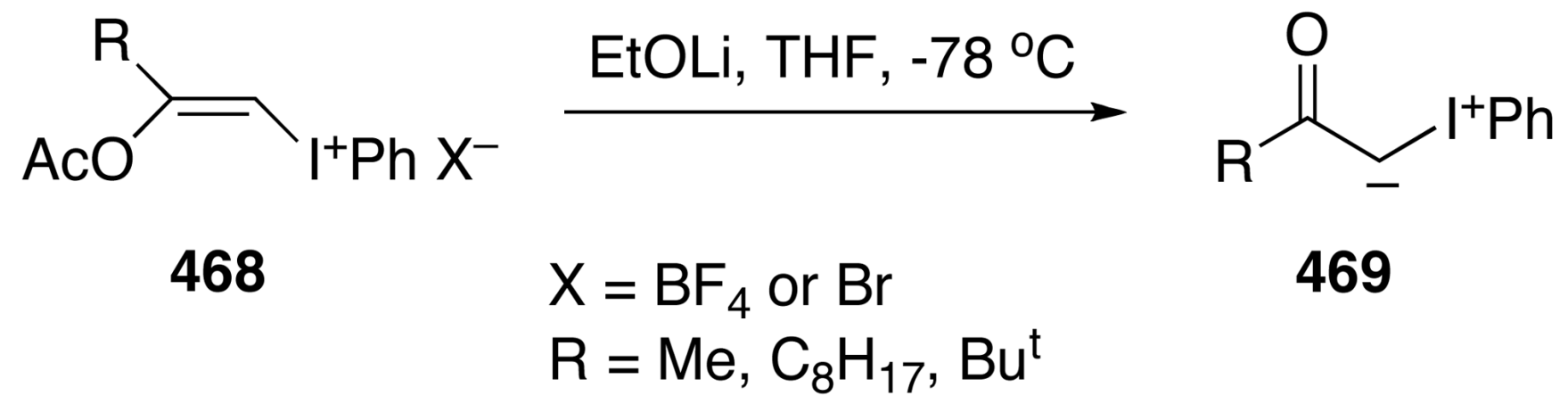

Scheme 153. 
<smiles>[R]c1cccs1</smiles>

470

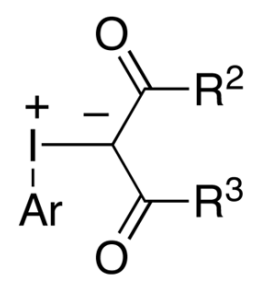

$\underset{15-39 \%}{\stackrel{\mathrm{BF}_{3} \cdot \mathrm{OEt}_{2}, \mathrm{CH}_{2} \mathrm{Cl}_{2}, \mathrm{rt}, 4-24 \mathrm{~h}}{\longrightarrow}}$

471

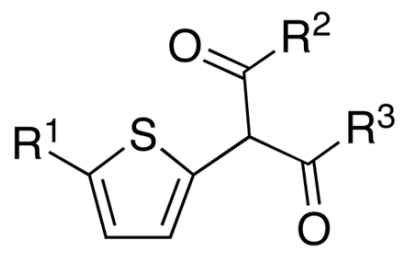

472

$$
\mathrm{R}^{1}=\mathrm{Me} \text { or } \mathrm{Et} ; \mathrm{R}^{2} / \mathrm{R}^{3}=\mathrm{OMe} / \mathrm{OMe}, \mathrm{Me} / \mathrm{OMe}, \mathrm{Ph} / \mathrm{Me}
$$

Scheme 154. 


\begin{tabular}{|c|c|c|c|}
\hline \multirow{2}{*}{$\stackrel{+}{\mathrm{I}}-\overline{\mathrm{C}}\left(\mathrm{SO}_{2} \mathrm{Ph}\right)_{2}$} & & MeCN, rt, in dark, $24 \mathrm{~h}$ & \multirow{2}{*}{$\mathrm{RCH}_{2}^{\mathrm{I}} \mathrm{C}\left(\mathrm{SO}_{2} \mathrm{Ph}\right)_{2}$} \\
\hline & $\begin{array}{c}+\mathrm{R} C \mathrm{H}_{2} \mathrm{I} \\
\mathbf{4 7 4}\end{array}$ & $24-56 \%$ & \\
\hline 473 & $\mathrm{R}=\mathrm{H}, \mathrm{\Lambda}$ & & 475 \\
\hline
\end{tabular}

Scheme 155. 
<smiles>[R]N([R])C(O[TlH])=C1C(=O)c2ccccc2C1=O</smiles>

\section{$\mathrm{R} / \mathrm{R}^{1}=\mathrm{Ph} / \mathrm{H}, \mathrm{Tol} / \mathrm{H}, 4-\mathrm{MeOC}_{6} \mathrm{H}_{4} / \mathrm{H}, 4-\mathrm{NO}_{2} \mathrm{C}_{6} \mathrm{H}_{4} / \mathrm{H}, 2-\mathrm{MeC}_{6} \mathrm{H}_{4} / \mathrm{H}$, $\mathrm{PhCH}_{2} / \mathrm{H}, \mathrm{PhCH}_{2} \mathrm{CH}_{2} / \mathrm{H}, \mathrm{Ph} / \mathrm{Me}, \mathrm{Ph} / \mathrm{Ph}$}

Scheme 156. 
<smiles>[R]c1cc([AsH2])c([OH+])c([R9])c1[R]</smiles>

478<smiles>[R7]C(=O)C([I-][PH2+])C([R5])=O</smiles>

479
CsF, MeCN, rt, 2.5-15 h<smiles>[AsH2]O[SbH]</smiles><smiles>[R]c1cc2c([R5])c([R7])oc2c([R3])c1[R]</smiles>

480

$\mathrm{R}^{1}-\mathrm{R}^{3}=\mathrm{H}, \mathrm{Me}, \mathrm{Bu}^{\mathrm{t}}$, etc.

$\mathrm{R}^{4} / \mathrm{R}^{5}=\mathrm{Me} / \mathrm{OMe}, \mathrm{Me} / \mathrm{OEt}, \mathrm{Me} / \mathrm{OPr}{ }^{\mathrm{i}}, \mathrm{Me} / \mathrm{OBn}, \mathrm{Et} / \mathrm{OMe}$, $\mathrm{Pr}^{\prime} / \mathrm{OMe}, \mathrm{ClCH}_{2} / \mathrm{OEt}$, Pr/OEt, Me/Me, Me/Ph, etc.

Scheme 157. 


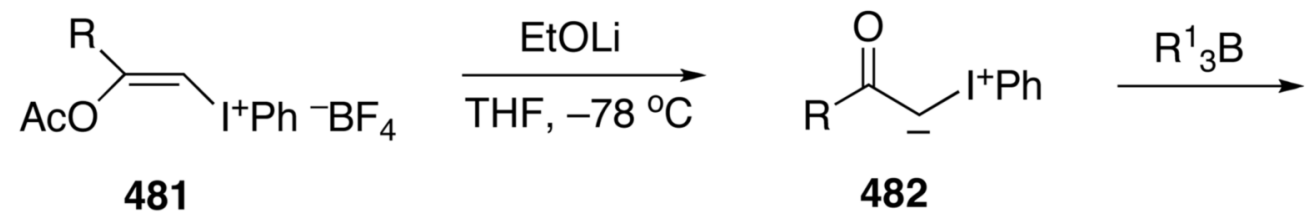

$$
\begin{aligned}
\mathrm{R}= & \mathrm{C}_{8} \mathrm{H}_{17}, \mathrm{Ph}\left(\mathrm{CH}_{2}\right)_{3}, \mathrm{Bu}^{\mathrm{t}} \\
\mathrm{R}^{1}= & \mathrm{Et}, \mathrm{Bu}, \mathrm{Bu}^{\mathrm{s}}, \mathrm{Ph}\left(\mathrm{CH}_{2}\right)_{3}, \\
& \text { cyclohexyl, } \mathrm{Ph}, \\
& \text { cyclopentyl, Tol }
\end{aligned}
$$<smiles>[R]C(=O)C([R7])[R7]</smiles><smiles>[R]CC([R])=O</smiles>

483

$50-96 \%$

Scheme 158. 


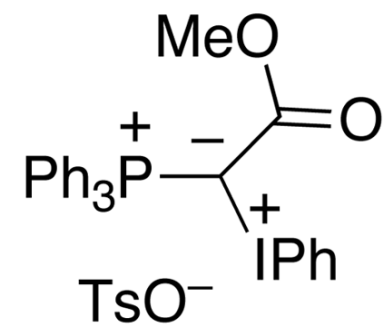

485

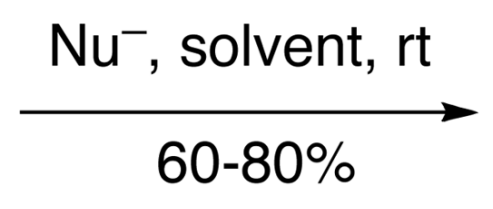

$\mathrm{Nu}=\mathrm{I}, \mathrm{Br}, \mathrm{PhS}, \mathrm{PhSO}_{2}$, etc.

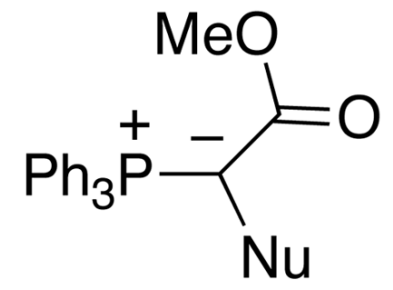

486

Scheme 159. 


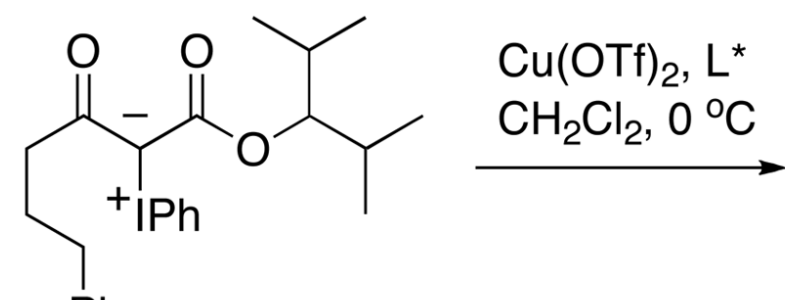

$\mathrm{Ph}$<smiles>CC(C)C(OC(=O)[C@@H]1C(=O)CC[C@H]1Pc1ccccc1)C(C)C</smiles>

$\underset{52 \%}{\stackrel{\mathrm{HBr}, \mathrm{EtOH}}{\longrightarrow}}$

488, $72 \%$ ee

Scheme 160. 
<smiles>[R]c1ccc2oc(=O)c([In+]c3ccccc3)c([O-])c2c1</smiles>

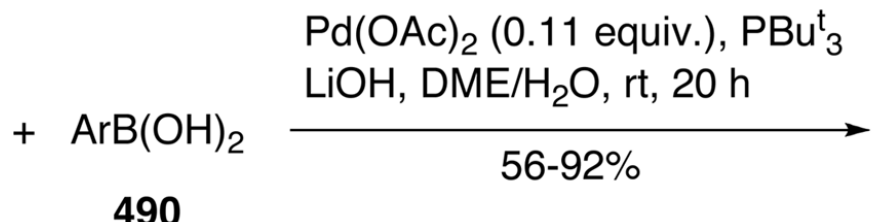

490<smiles>[R]c1ccc2oc(=O)c(Br)c(O)c2c1</smiles>

491

$\mathrm{R}=\mathrm{H}, \mathrm{Me}, \mathrm{Cl}, \mathrm{F}$, etc.

$\mathrm{Ar}=\mathrm{Ph}, 4-\mathrm{MeOC}_{6} \mathrm{H}_{4}, 2-\mathrm{MeOC}_{6} \mathrm{H}_{4}, 4-\mathrm{ClC}_{6} \mathrm{H}_{4}, 4-\mathrm{FC}_{6} \mathrm{H}_{4}$, etc.

Scheme 161. 
$\mathrm{Arl}(\mathrm{OAC})_{2}+\mathrm{H}_{2} \mathrm{NR}$ 492 493

\section{$\mathrm{KOH}, \mathrm{MeOH}$ $60-97 \%$}

Arl $=\mathrm{NR}$

494

$$
\begin{aligned}
& \mathrm{Ar}=\mathrm{Ph}, 3-\mathrm{MeC}_{6} \mathrm{H}_{4}, 2,4,6-\mathrm{Me}_{3} \mathrm{C}_{6} \mathrm{H}_{2} \text {, etc. } \\
& \mathrm{R}=\mathrm{Ts}, 4-\mathrm{MeOC}_{6} \mathrm{H}_{4} \mathrm{SO}_{2}, 4-\mathrm{CF}_{3} \mathrm{C}_{6} \mathrm{H}_{4} \mathrm{SO}_{2} \text {, }
\end{aligned}
$$
4- $\mathrm{NO}_{2} \mathrm{C}_{6} \mathrm{H}_{4} \mathrm{SO}_{2}, 2-\mathrm{NO}_{2} \mathrm{C}_{6} \mathrm{H}_{4} \mathrm{SO}_{2}, 4-\mathrm{FC}_{6} \mathrm{H}_{4} \mathrm{SO}_{2}$, $4-\mathrm{IC}_{6} \mathrm{H}_{4} \mathrm{SO}_{2}, \mathrm{PhSO}_{2}, \mathrm{MeSO}_{2}, \mathrm{CF}_{3} \mathrm{CO}$, etc.

Scheme 162. 


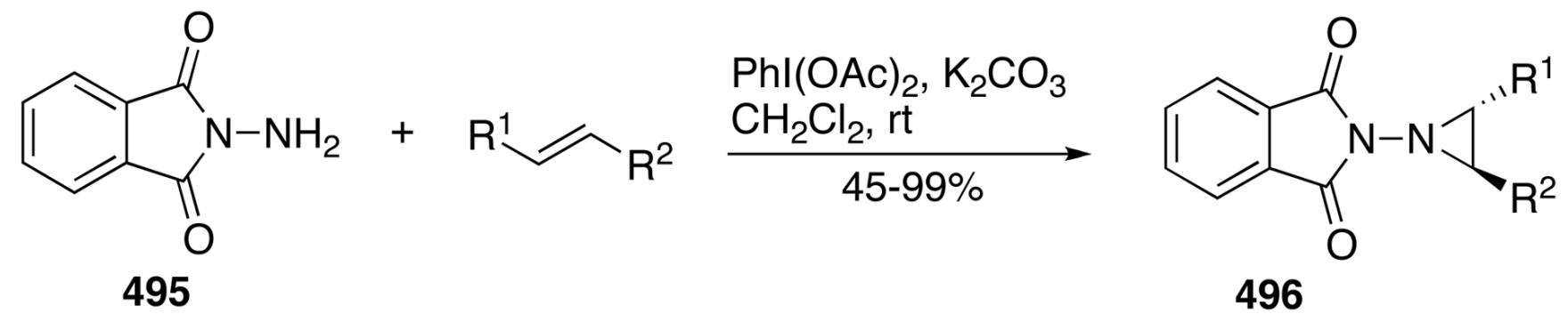

$\mathrm{R}^{1} / \mathrm{R}^{2}=\mathrm{Ph} / \mathrm{H}, 4-\mathrm{CF}_{3} \mathrm{C}_{6} \mathrm{H}_{4} / \mathrm{H}, 4-\mathrm{FC}_{6} \mathrm{H}_{4} / \mathrm{H}, 4-\mathrm{MeC}_{6} \mathrm{H}_{4} / \mathrm{H}, \mathrm{Ph} / \mathrm{Me}, \mathrm{Ph} / \mathrm{CO}_{2} \mathrm{Me}$, etc. Scheme 163. 


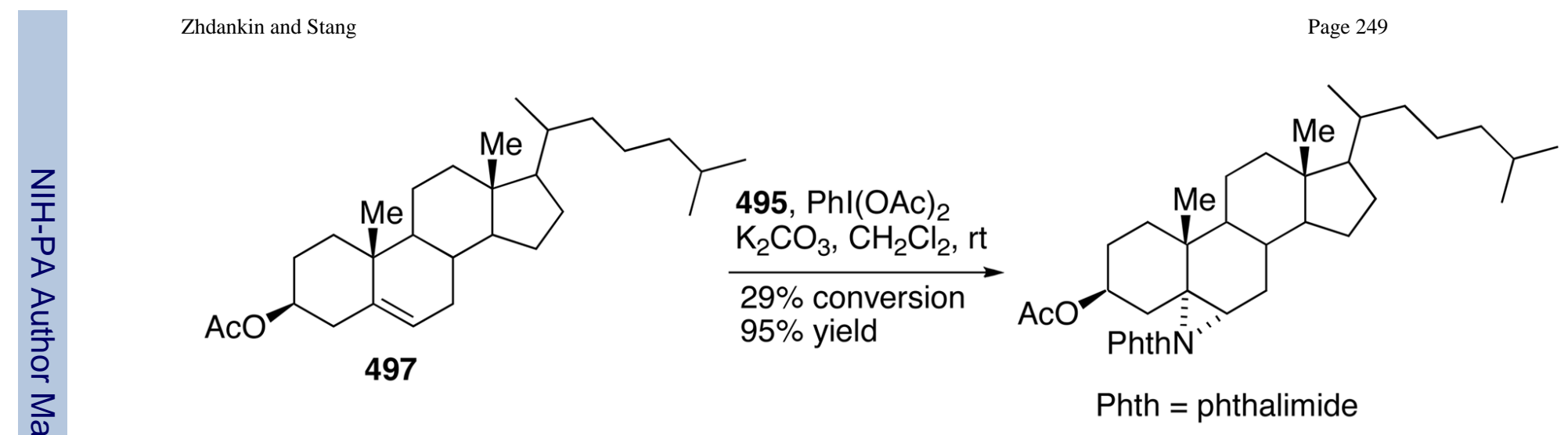

Scheme 164. 


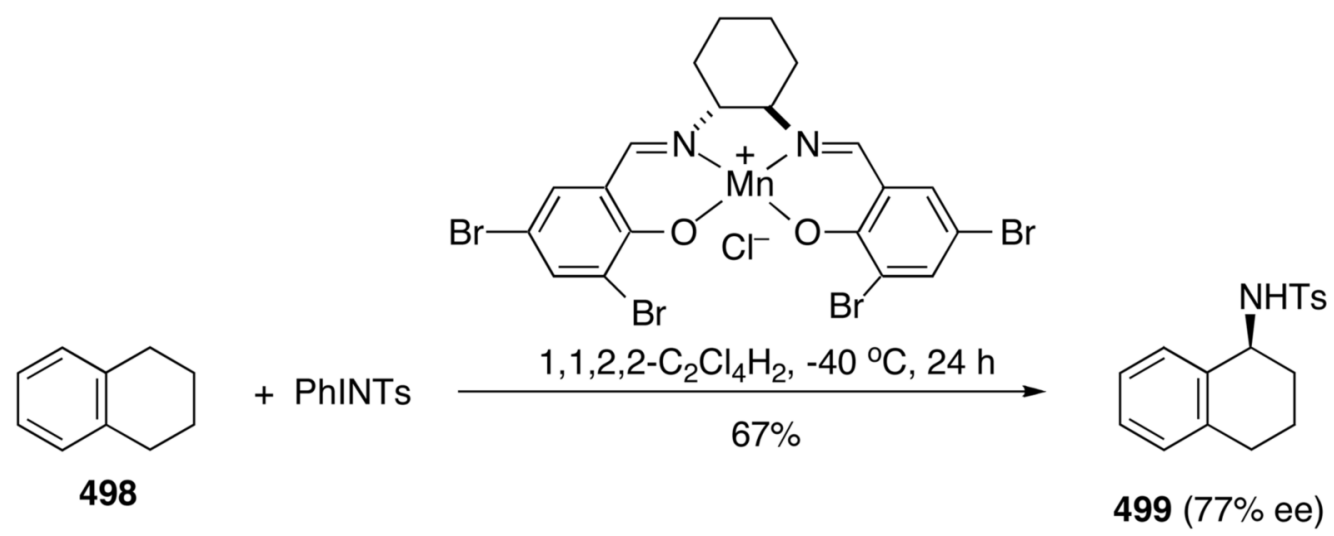

Scheme 165. 


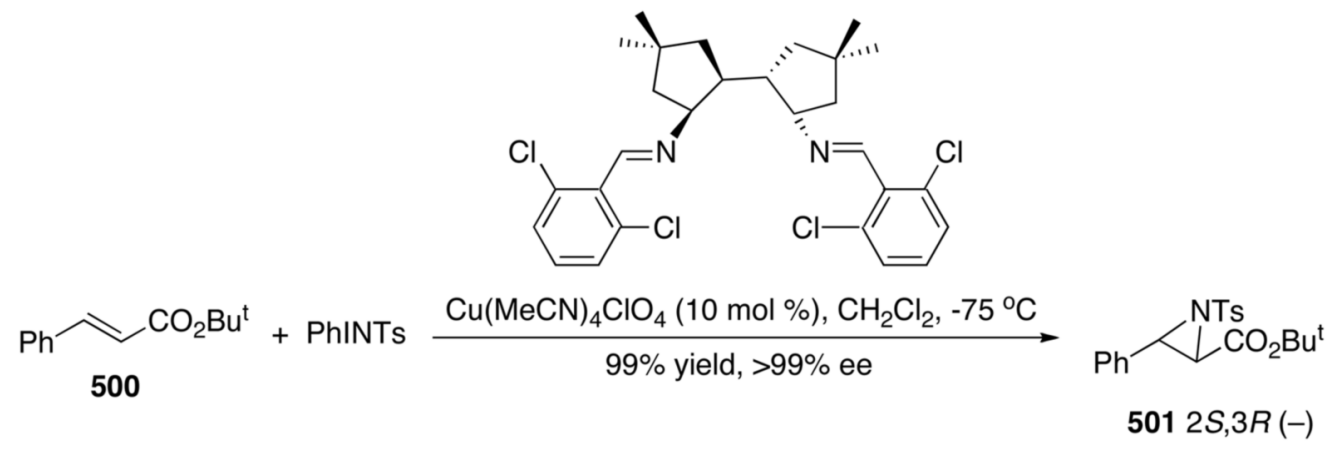

Scheme 166. 
$\mathrm{Rh}_{2}(S-T F P T T L)_{4}(3 \mathrm{~mol} \%)$<smiles>[R]C=C(C)O[GaH]</smiles>

$\mathrm{CH}_{2} \mathrm{Cl}_{2},-40{ }^{\circ} \mathrm{C}, 3-24 \mathrm{~h}$

503

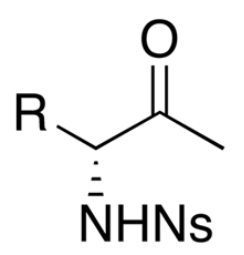

504

$\mathrm{R}=\mathrm{Ph}, 4-\mathrm{ClC}_{6} \mathrm{H}_{4}, 4-\mathrm{MeOC}_{6} \mathrm{H}_{4}, \mathrm{PhCH}_{2}, 4-\mathrm{ClC}_{6} \mathrm{H}_{4} \mathrm{CH}_{2}, 4-\mathrm{MeOC}_{6} \mathrm{H}_{4} \mathrm{CH}_{2}$, etc. $\mathrm{Ns}=2-\mathrm{NO}_{2} \mathrm{C}_{6} \mathrm{H}_{4} \mathrm{SO}_{2}$

Scheme 167. 
<smiles></smiles>

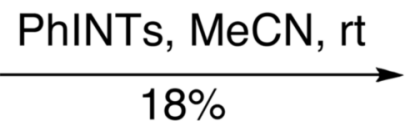

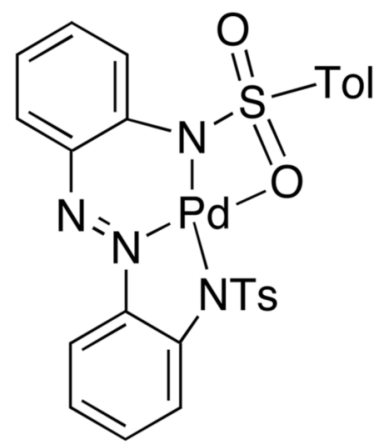

\section{5}

Scheme 168. 


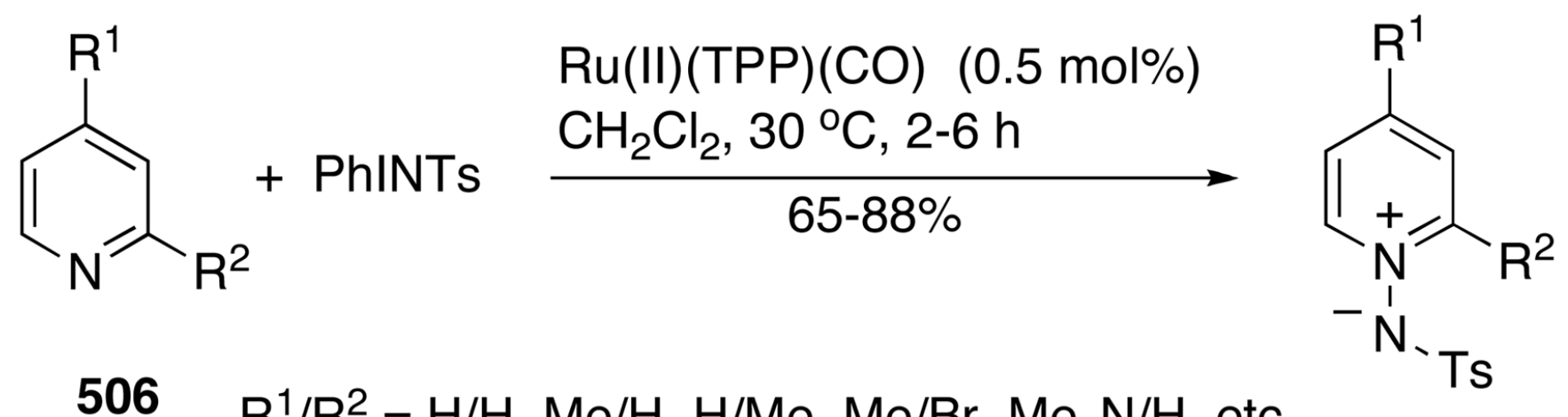

Scheme 169. 
$\mathrm{Fe}(\mathrm{acac})_{3}(10 \mathrm{~mol} \%)$

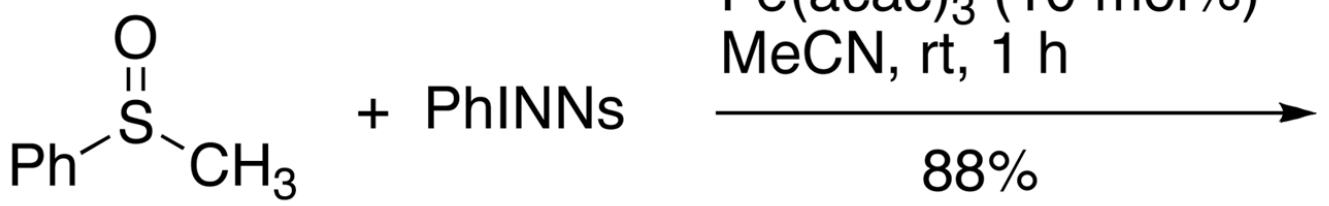<smiles>CS(C)(=N[SH3])=NS(=O)(=O)c1ccccc1</smiles>

507

Scheme 170. 


$$
\begin{aligned}
& \mathrm{Arl}+\mathrm{NalO}_{4} \underset{30-91 \%}{\stackrel{30 \% \text { aq. AcOH, reflux, 3-6 h }}{\longrightarrow}} \mathrm{ArlO}_{2} \\
& 508 \\
& 509
\end{aligned}
$$

$$
\begin{aligned}
\mathrm{Ar}= & \mathrm{Ph}, 4-\mathrm{MeC}_{6} \mathrm{H}_{4}, 3-\mathrm{MeC}_{6} \mathrm{H}_{4}, 2-\mathrm{MeC}_{6} \mathrm{H}_{4}, 4-\mathrm{FC}_{6} \mathrm{H}_{4}, 3-\mathrm{FC}_{6} \mathrm{H}_{4}, \\
& 4-\mathrm{ClC}_{6} \mathrm{H}_{4}, 3-\mathrm{ClC}_{6} \mathrm{H}_{4}, 2-\mathrm{ClC}_{6} \mathrm{H}_{4}, 4-\mathrm{BrC}_{6} \mathrm{H}_{4}, 3-\mathrm{NO}_{2} \mathrm{C}_{6} \mathrm{H}_{4}, \\
& 2-\mathrm{NO}_{2} \mathrm{C}_{6} \mathrm{H}_{4}, 4-\mathrm{NO}_{2} \mathrm{C}_{6} \mathrm{H}_{4}, 2,4-\mathrm{Me}_{2} \mathrm{C}_{6} \mathrm{H}_{3}, 2,4-\mathrm{Cl}_{2} \mathrm{C}_{6} \mathrm{H}_{3}
\end{aligned}
$$

Scheme 171. 


\section{$\mathrm{RuCl}_{3}(0.08 \mathrm{~mol} \%), 40^{\circ} \mathrm{C}, 16 \mathrm{~h}$ \\ $\mathrm{Arl}+\mathrm{AcOOH} / \mathrm{AcOH}$ \\ 508 \\ $64-94 \%$ \\ $\mathrm{ArlO}_{2}$ \\ 509
$\mathrm{R}=\mathrm{H}, 4-\mathrm{MeC}_{6} \mathrm{H}_{4}, 2-\mathrm{MeC}_{6} \mathrm{H}_{4}, 2-\mathrm{ClC}_{6} \mathrm{H}_{4}, 3-\mathrm{ClC}_{6} \mathrm{H}_{4}, 4-\mathrm{ClC}_{6} \mathrm{H}_{4}, 4-\mathrm{BrC}_{6} \mathrm{H}_{4}$, $4-\mathrm{C}_{6} \mathrm{H}_{4} \mathrm{~F}, 4-\mathrm{CF}_{3} \mathrm{C}_{6} \mathrm{H}_{4}, 3,5-\mathrm{CF}_{3} \mathrm{C}_{6} \mathrm{H}_{3}$, etc.

Scheme 172. 
<smiles>O=P(c1ccccc1)(c1ccccc1)c1ccccc1I</smiles>

$\mathrm{NaOCl}(5.25 \%$ aq.) $\mathrm{Bu}_{4} \mathrm{NBr}, \mathrm{CH}_{2} \mathrm{Cl}_{2}$, rt $71 \%$<smiles></smiles>

511

Scheme 173. 


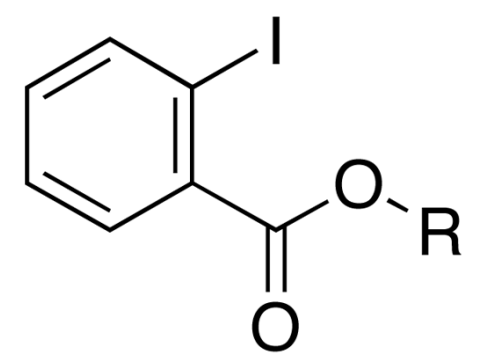

512
$\mathrm{NaOCl}, \mathrm{AcOH}, \mathrm{rt}$

$61-89 \%$

$\mathrm{R}=\mathrm{Me}, \mathrm{Et}, \operatorname{Pr}^{\mathrm{i}},(-)$-menthyl, (+)-menthyl, ( \pm )-menthyl, [(1S)-endo]-(-)-bornyl, 2-adamantyl, 1-adamantyl, $\mathrm{Bu}^{\mathrm{t}}$

Scheme 174. 
<smiles>[R]NC(=O)c1ccccc1I</smiles>

516

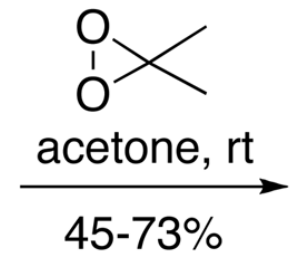

517

$\mathrm{R}=(\mathrm{S})-\mathrm{CH}\left(\mathrm{CH}_{3}\right) \mathrm{CO}_{2} \mathrm{CH}_{3},(R)-\mathrm{CH}\left(\mathrm{CH}_{3}\right) \mathrm{CO}_{2} \mathrm{CH}_{3},(S)-\mathrm{CH}\left(\mathrm{CH}_{2} \mathrm{Ph}\right) \mathrm{CO}_{2} \mathrm{CH}_{3}$, (S) $-\mathrm{CH}\left(\mathrm{Bu}^{\mathrm{i}}\right) \mathrm{CO}_{2} \mathrm{CH}_{3}, \mathrm{CH}_{2} \mathrm{CH}_{2} \mathrm{CO}_{2} \mathrm{H}, \mathrm{CH}\left(\mathrm{CH}_{3}\right) \mathrm{CH}_{2} \mathrm{CO}_{2} \mathrm{H},(R)-\mathrm{CH}(\mathrm{Ph}) \mathrm{CH}_{3}$

Scheme 175. 


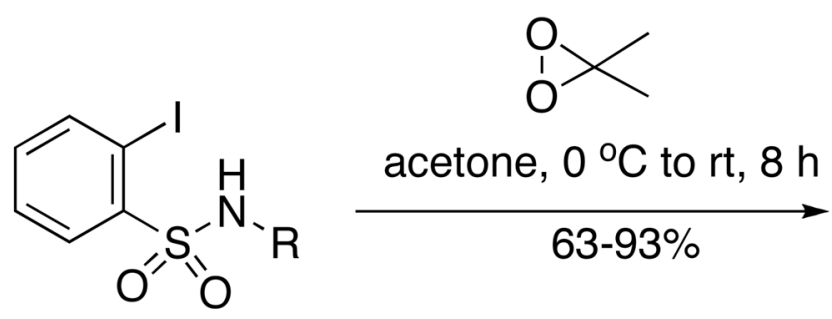

523<smiles></smiles>

524

$$
\begin{aligned}
\mathrm{R}= & (\mathrm{S})-\mathrm{CH}\left(\mathrm{CH}_{3}\right) \mathrm{CO}_{2} \mathrm{CH}_{3},(\mathrm{~S})-\mathrm{CH}\left(\mathrm{CH}_{2} \mathrm{Ph} \mathrm{CO}_{2} \mathrm{CH}_{3},\right. \\
& (S)-\mathrm{CH}(i-\mathrm{Pr}) \mathrm{CO}_{2} \mathrm{CH}_{3},(S)-\mathrm{CH}(i-\mathrm{Bu}) \mathrm{CO}_{2} \mathrm{CH}_{3},(R)-\mathrm{CH}(\mathrm{Ph}) \mathrm{CH}_{3}
\end{aligned}
$$

Scheme 176. 


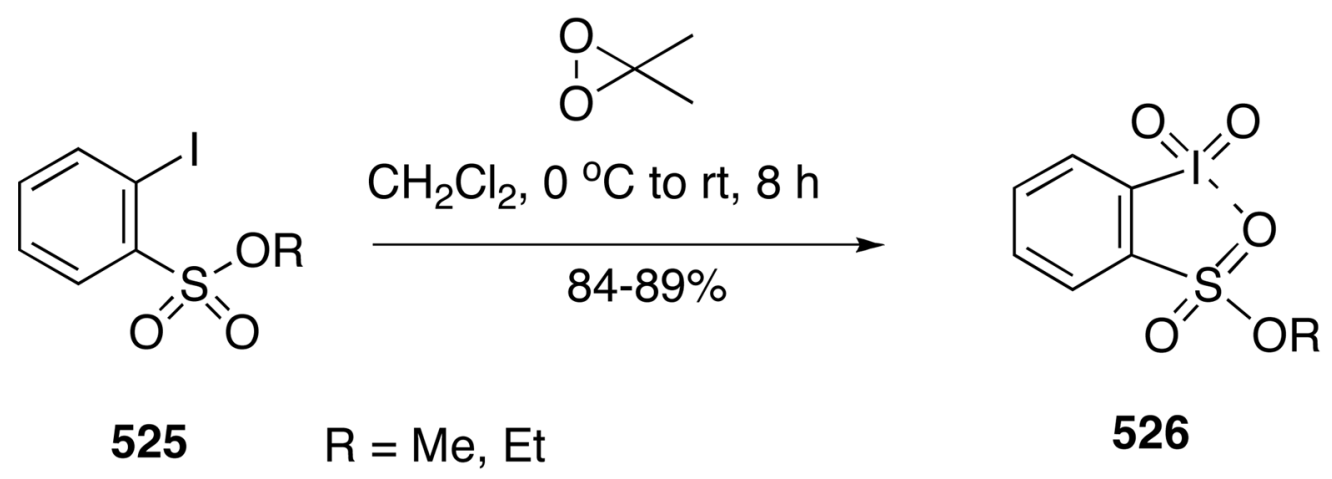

Scheme 177. 
<smiles>[R2]C(=O)N([R7])c1ccccc1I</smiles>

acetone $-\mathrm{CH}_{2} \mathrm{Cl}_{2}$, rt

$69-97 \%$

527

$$
\begin{aligned}
& \mathrm{R}^{1}=\mathrm{H}, \mathrm{Me}, \mathrm{Bn} \\
& \mathrm{R}^{2}=\mathrm{Me}, \operatorname{Pr}, \operatorname{Pr}^{\mathrm{i}} \text {, cyclohexyl, } \mathrm{Bu}^{\mathrm{t}} \text {, etc. }
\end{aligned}
$$
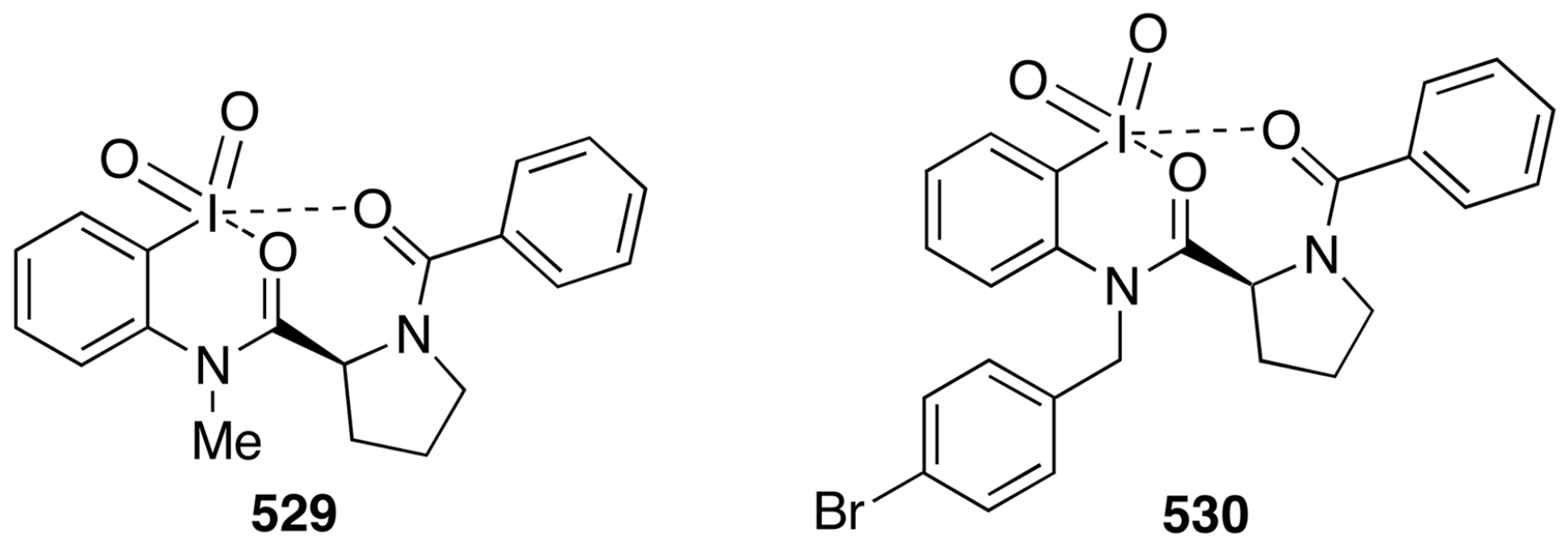

Scheme 178. 

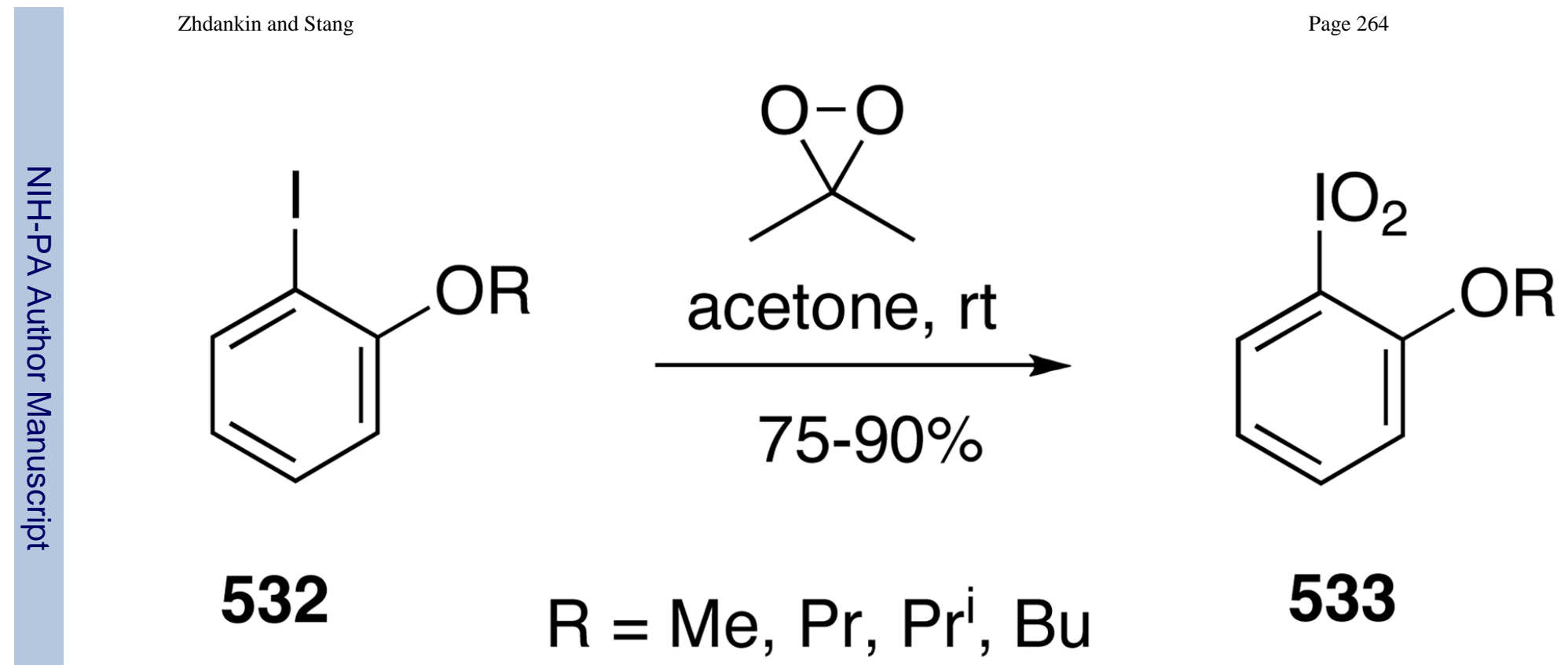

Scheme 179. 
<smiles>O=C(O)c1ccccc1I</smiles>

536

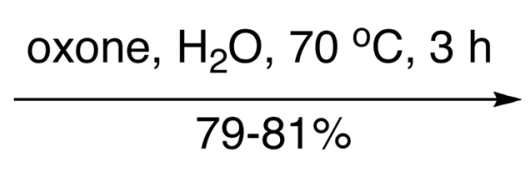

Scheme 180.

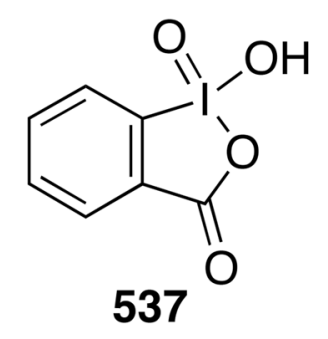


<smiles>COS(=O)(=O)c1ccccc1[N+](=O)[O-]</smiles>

543<smiles>O=S(=O)(O)c1ccccc1I</smiles>

544

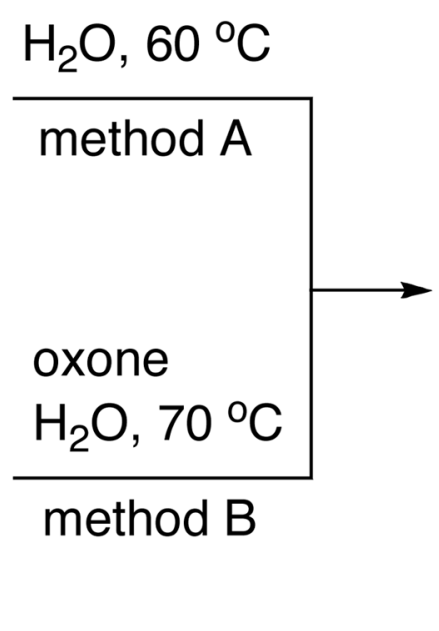<smiles>O=P1(O)OS(=O)(=O)c2ccccc21</smiles>

545

Scheme 181. 


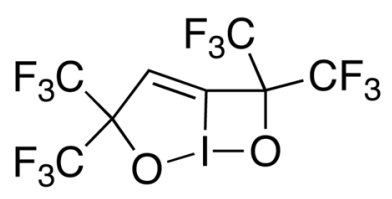

546
1. $\mathrm{XeF}_{2}, \mathrm{MeCN}, 70^{\circ} \mathrm{C}$

2. aerial humidity $30 \%$

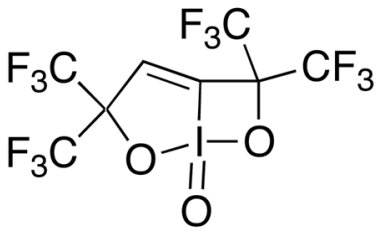

547

Scheme 182. 
$\stackrel{\mathrm{IBX}, \mathrm{MeCN}, 80^{\circ} \mathrm{C}, 75 \mathrm{~min}}{\longrightarrow}$ $93 \%$

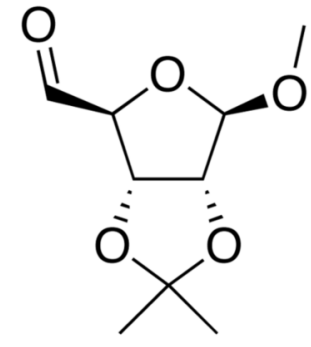

553

Scheme 183. 

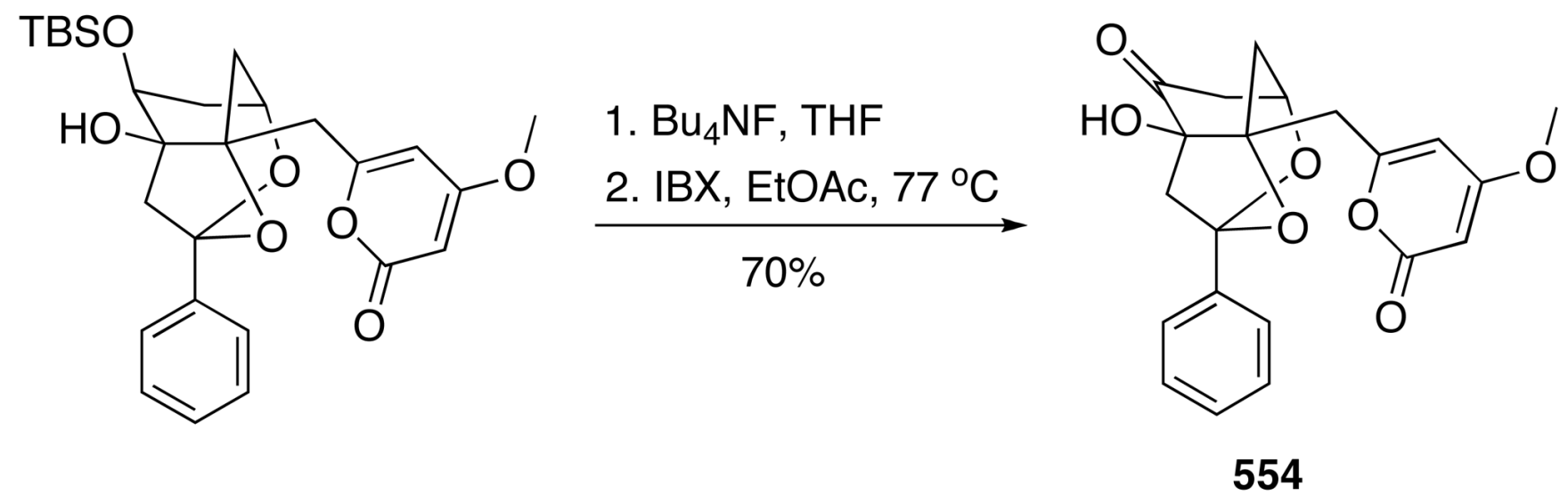

Scheme 184. 
<smiles>[R]CO</smiles>
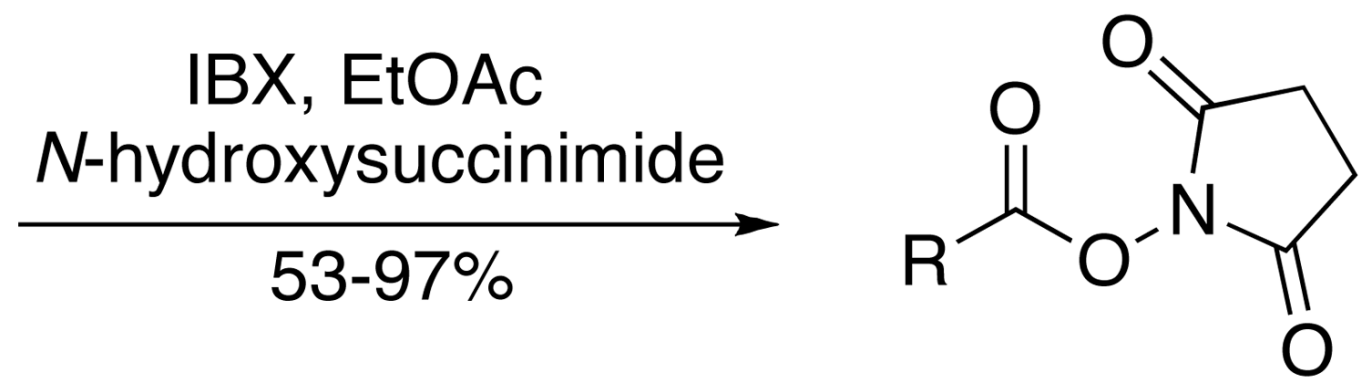<smiles>[R]C=O</smiles>

555

Scheme 185. 

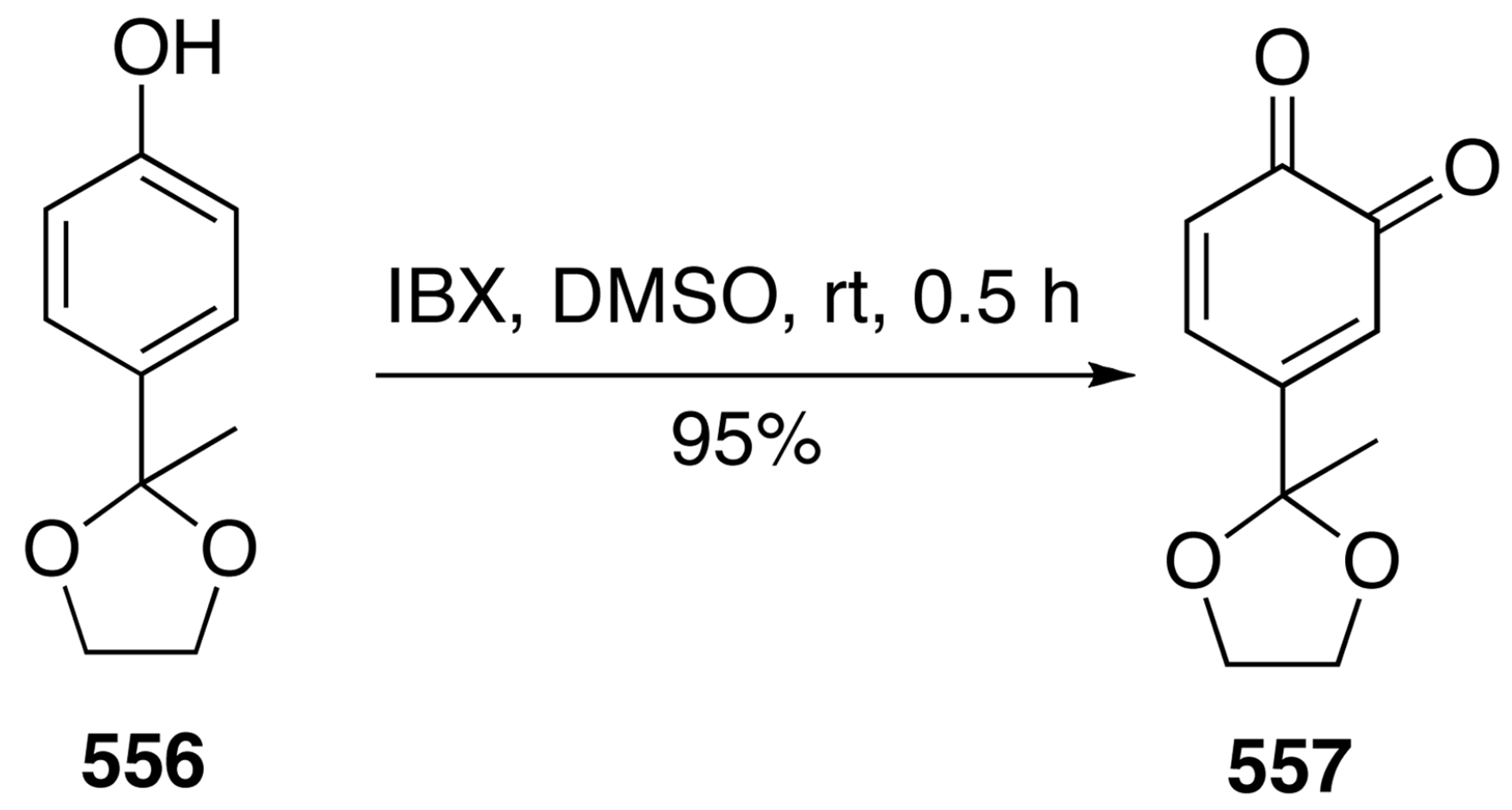

Scheme 186.

557

Scheme 186. 


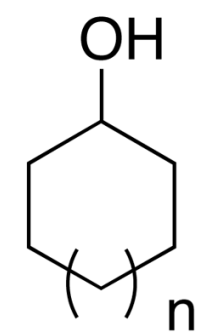

\section{IBX, fluorobenzene/DMSO, 60-65 ${ }^{\circ} \mathrm{C}, 2-24 \mathrm{~h}$ $83-88 \%$}

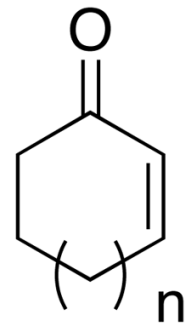

559

Scheme 187. 


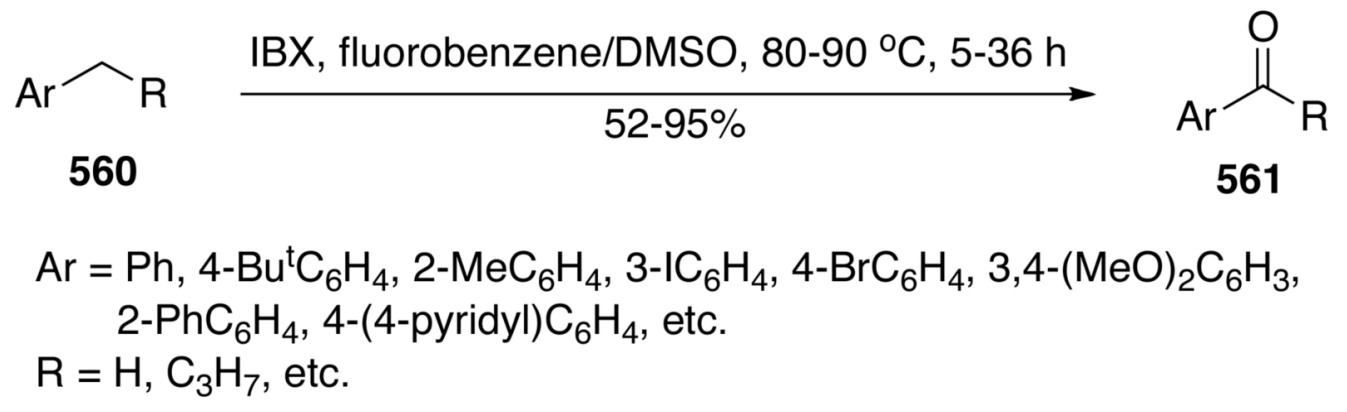

Scheme 188. 


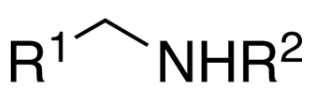

562
IBX, DMSO, $25-45^{\circ} \mathrm{C}, 10$ - $840 \mathrm{~min}$ $61-99 \%$

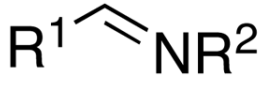

563

$\mathrm{R}^{1}=\mathrm{Ph}, 4-\mathrm{BrC}_{6} \mathrm{H}_{4}, 4-\mathrm{MeOC}_{6} \mathrm{H}_{4}$, etc.

$\mathrm{R}^{2}=4-\mathrm{BrC}_{6} \mathrm{H}_{4}, 4-\mathrm{MeOC}_{6} \mathrm{H}_{4}, \mathrm{CH}_{3}, \mathrm{OH}, \mathrm{OBn}$, etc.

Scheme 189. 


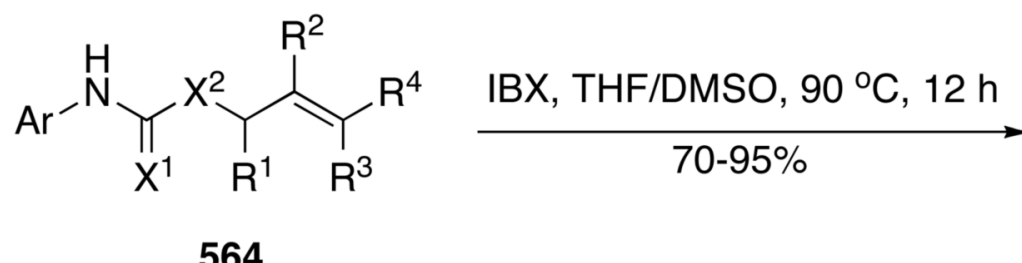

564<smiles>[Y]C([R])N([Al])C([R])([R])C([R])[Y]</smiles>

565

$\mathrm{X}^{1}=\mathrm{O}, \mathrm{S} ; \mathrm{X}^{2}=\mathrm{CH}_{2}, \mathrm{O}, \mathrm{N}$

$\mathrm{Ar}=\mathrm{Ph}, 3-\mathrm{EtC}_{6} \mathrm{H}_{4}, 3-\mathrm{BrC}_{6} \mathrm{H}_{4}, 3-\mathrm{FC}_{6} \mathrm{H}_{4}, 4-\mathrm{EtC}_{6} \mathrm{H}_{4}$, etc. $\mathrm{R}^{1}-\mathrm{R}^{4}=\mathrm{H}$, alkyl, cycloalkyl, etc.

Scheme 190. 

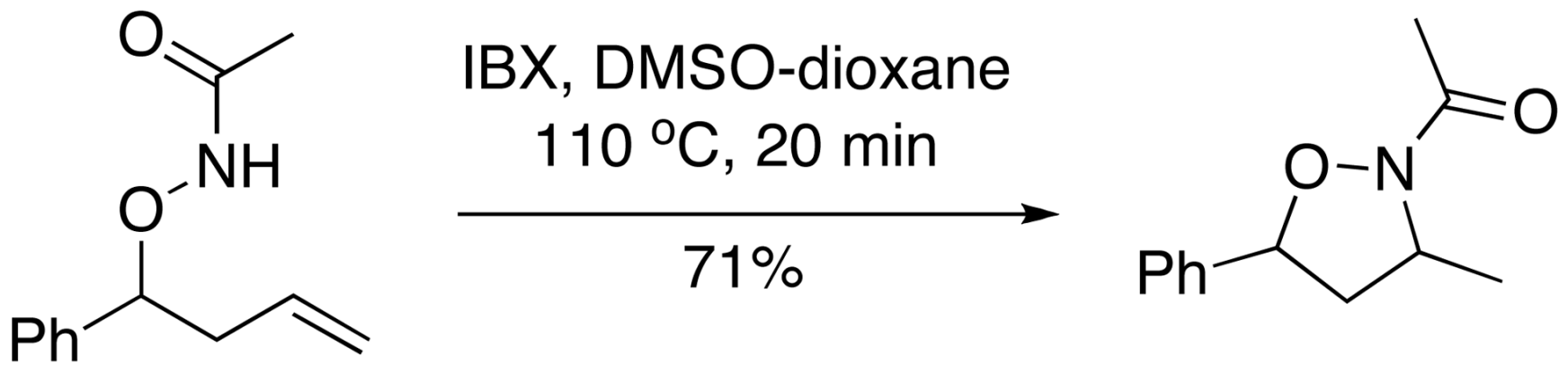

566

Scheme 191. 


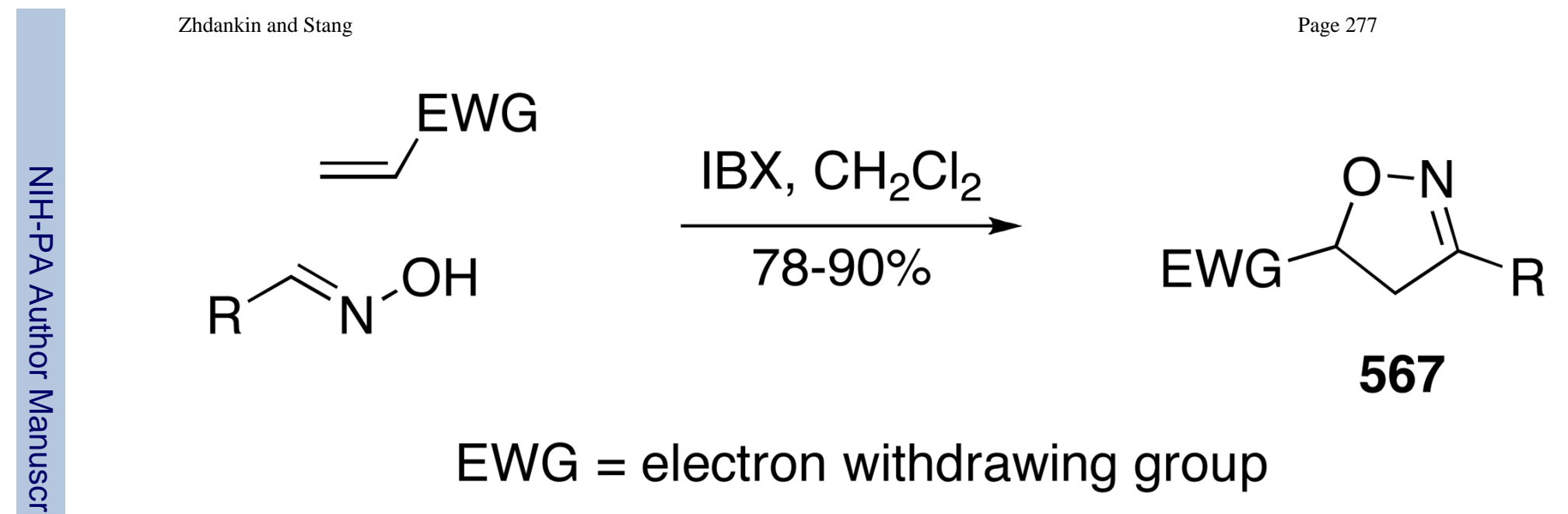

Scheme 192. 
$\mathrm{R}^{1} \stackrel{\mathrm{O}}{\mathrm{H}}_{\mathrm{H}}+\mathrm{R}^{2} \mathrm{NH}_{2}+\mathrm{TMSCN} \frac{\mathrm{IBX} / \mathrm{Bu}_{4} \mathrm{NBr}, \mathrm{MeCN}, \mathrm{rt}}{55-99 \%}$

$\mathrm{R}^{1}=\mathrm{Ph}, 4-\mathrm{MeOC}_{6} \mathrm{H}_{4}, 2,6-\mathrm{Cl}_{2} \mathrm{C}_{6} \mathrm{H}_{3}, \mathrm{PhC}=\mathrm{CH}, \mathrm{Ph}\left(\mathrm{CH}_{2}\right)_{2}, \mathrm{Pr}^{\mathrm{i}}$, etc.

$\mathrm{R}^{2}=\mathrm{Ph}\left(\mathrm{CH}_{2}\right)_{2}, \mathrm{Bu}^{\mathrm{t}}, 4-\mathrm{MeOC}_{6} \mathrm{H}_{4}, \mathrm{Ph}$, etc.

Scheme 193. 


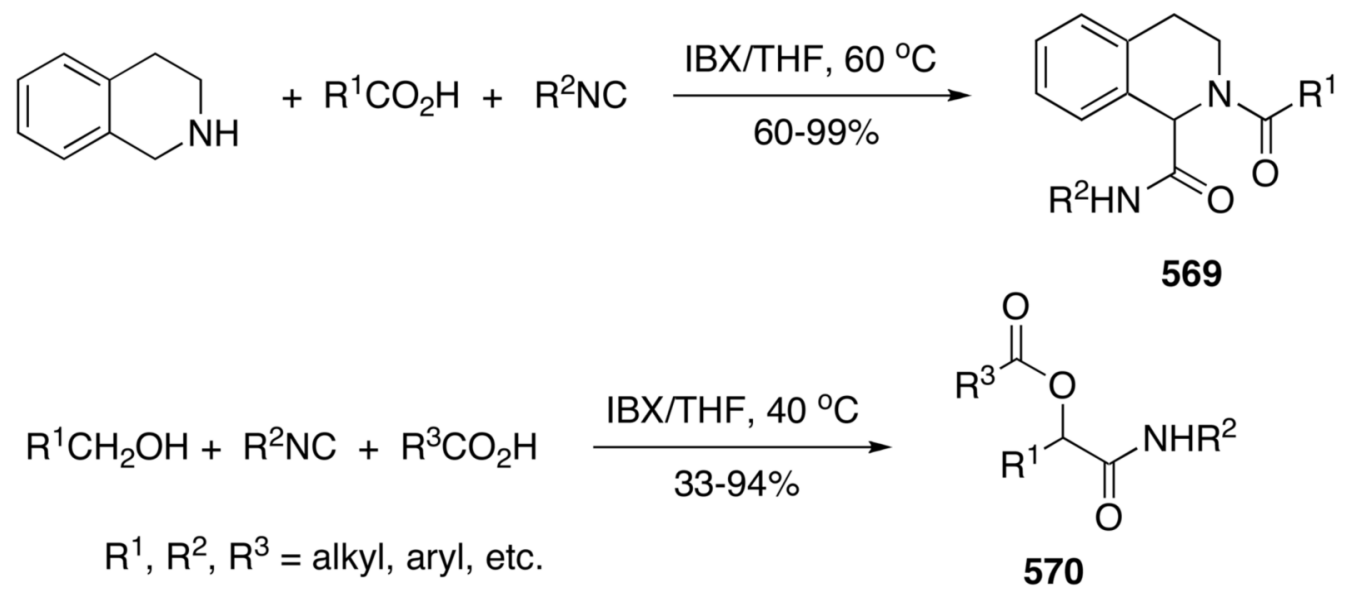

Scheme 194. 
<smiles>O=C1OI(=O)(O)c2ccccc21</smiles>

$\frac{\mathrm{Ac}_{2} \mathrm{O}, 0.5 \% \mathrm{TsOH}, 80{ }^{\circ} \mathrm{C}, 2 \mathrm{~h}}{91 \%}$

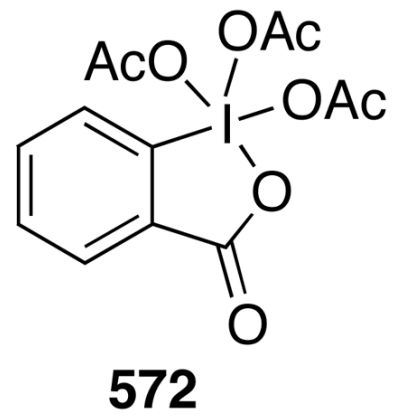

Scheme 195. 
<smiles>CC(C)(C)O[C@H]1CCCC2=CC(O)C(O)C[C@]2(C)[C@H]1OC(=O)O</smiles>

Scheme 196. 


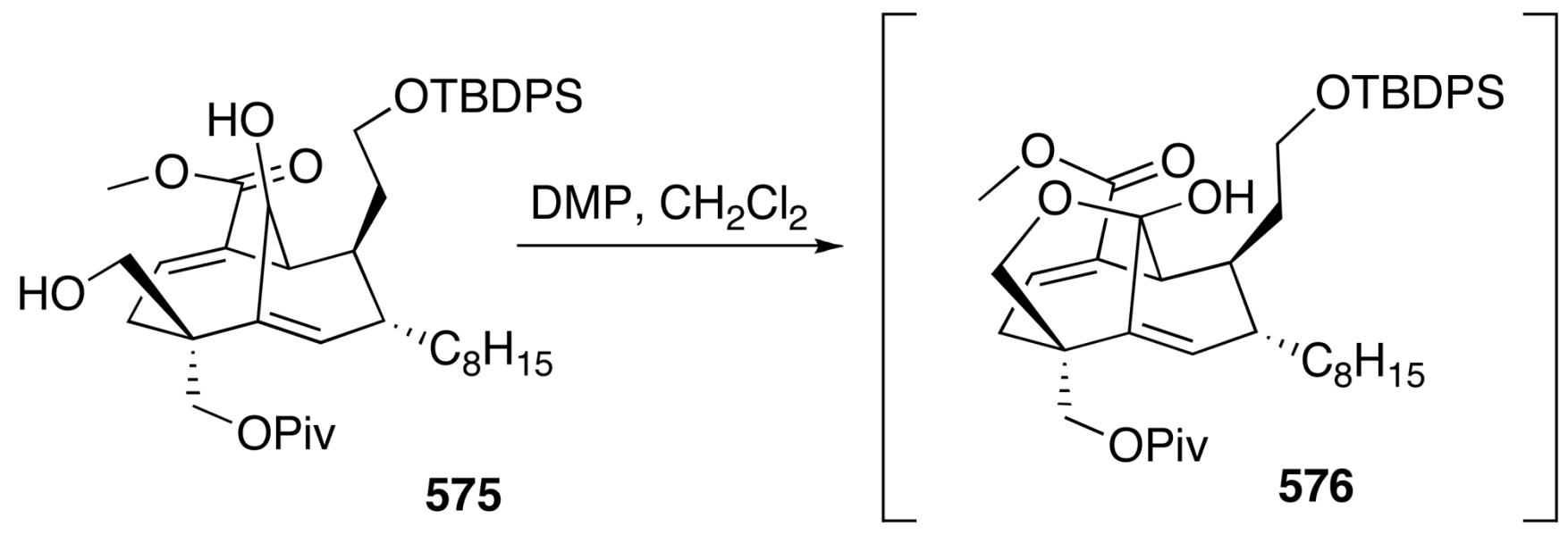

DMP, $\mathrm{CH}_{2} \mathrm{Cl}_{2}$

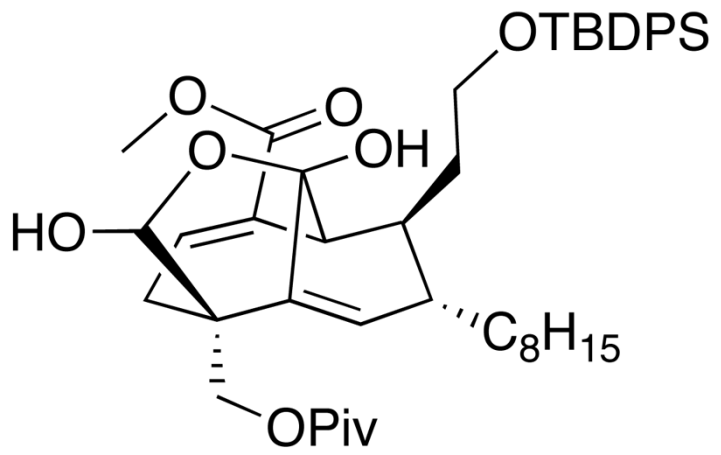

577

Scheme 197. 
<smiles>[R][R]1cccc(C(=S)Nc2cc[R17]cc2)c1</smiles>

578
DMP, $\mathrm{CH}_{2} \mathrm{Cl}_{2}$

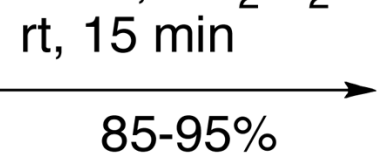

579<smiles>[R2][X]1ccccc1-c1nc2c(s1)C=C[R17]C=C2</smiles>

$\mathrm{R}^{2}$

$$
\mathrm{R}^{1}, \mathrm{R}^{2}=\text { alkyl, acyl, aryl, etc. }
$$

Scheme 198. 


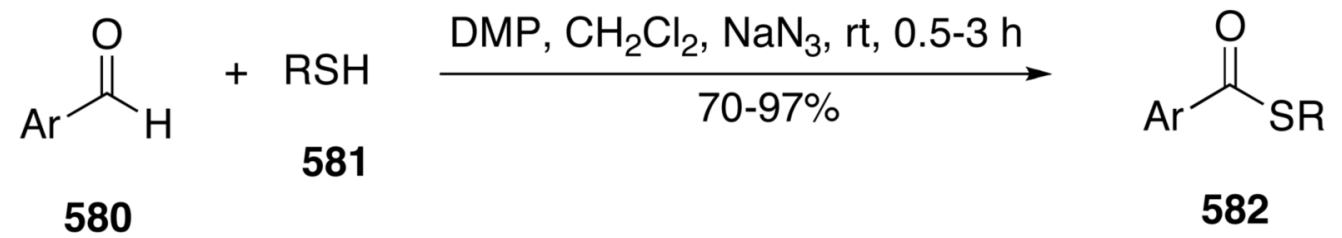

$$
\begin{aligned}
& \mathrm{Ar}=4-\mathrm{MeOC}_{6} \mathrm{H}_{4}, 3,4-(\mathrm{MeO})_{2} \mathrm{C}_{6} \mathrm{H}_{3}, 3,4,5-(\mathrm{MeO})_{3} \mathrm{C}_{6} \mathrm{H}_{2}, 4-\mathrm{MeC}_{6} \mathrm{H}_{4} \text {, } \\
& \text { 4- }\left(\mathrm{NMe}_{2}\right) \mathrm{C}_{6} \mathrm{H}_{4}, 4-\mathrm{ClC}_{6} \mathrm{H}_{4}, 4-\mathrm{NO}_{2} \mathrm{C}_{6} \mathrm{H}_{4}, 3-\mathrm{NO}_{2} \mathrm{C}_{6} \mathrm{H}_{4} \text {, etc. } \\
& \mathrm{R}=\mathrm{Ph}, \mathrm{Tol}
\end{aligned}
$$

Scheme 199. 


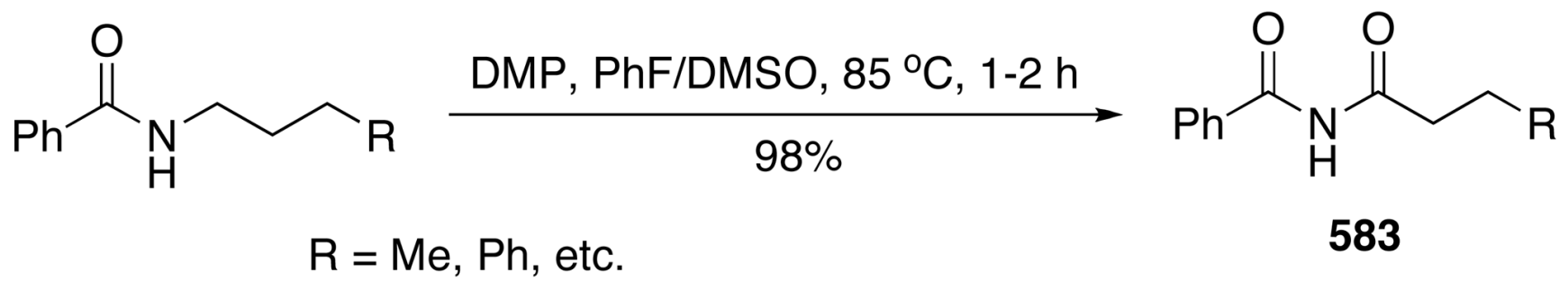

Scheme 200. 